\title{
Evaluating health promotion in complex adaptive school systems
}

Citation for published version (APA):

Bartelink, N. (2019). Evaluating health promotion in complex adaptive school systems: The Healthy Primary School of the Future. [Doctoral Thesis, Maastricht University]. ProefschriftMaken. https://doi.org/10.26481/dis.20191030nb

Document status and date:

Published: 01/01/2019

DOI:

10.26481/dis.20191030nb

Document Version:

Publisher's PDF, also known as Version of record

\section{Please check the document version of this publication:}

- A submitted manuscript is the version of the article upon submission and before peer-review. There can be important differences between the submitted version and the official published version of record.

People interested in the research are advised to contact the author for the final version of the publication, or visit the DOI to the publisher's website.

- The final author version and the galley proof are versions of the publication after peer review.

- The final published version features the final layout of the paper including the volume, issue and page numbers.

Link to publication

\footnotetext{
General rights rights.

- You may freely distribute the URL identifying the publication in the public portal. please follow below link for the End User Agreement:

www.umlib.nl/taverne-license

Take down policy

If you believe that this document breaches copyright please contact us at:

repository@maastrichtuniversity.nl

providing details and we will investigate your claim.
}

Copyright and moral rights for the publications made accessible in the public portal are retained by the authors and/or other copyright owners and it is a condition of accessing publications that users recognise and abide by the legal requirements associated with these

- Users may download and print one copy of any publication from the public portal for the purpose of private study or research.

- You may not further distribute the material or use it for any profit-making activity or commercial gain

If the publication is distributed under the terms of Article $25 \mathrm{fa}$ of the Dutch Copyright Act, indicated by the "Taverne" license above, 
Evaluating health promotion in complex adaptive school systems:

The Healthy Primary School of the Future

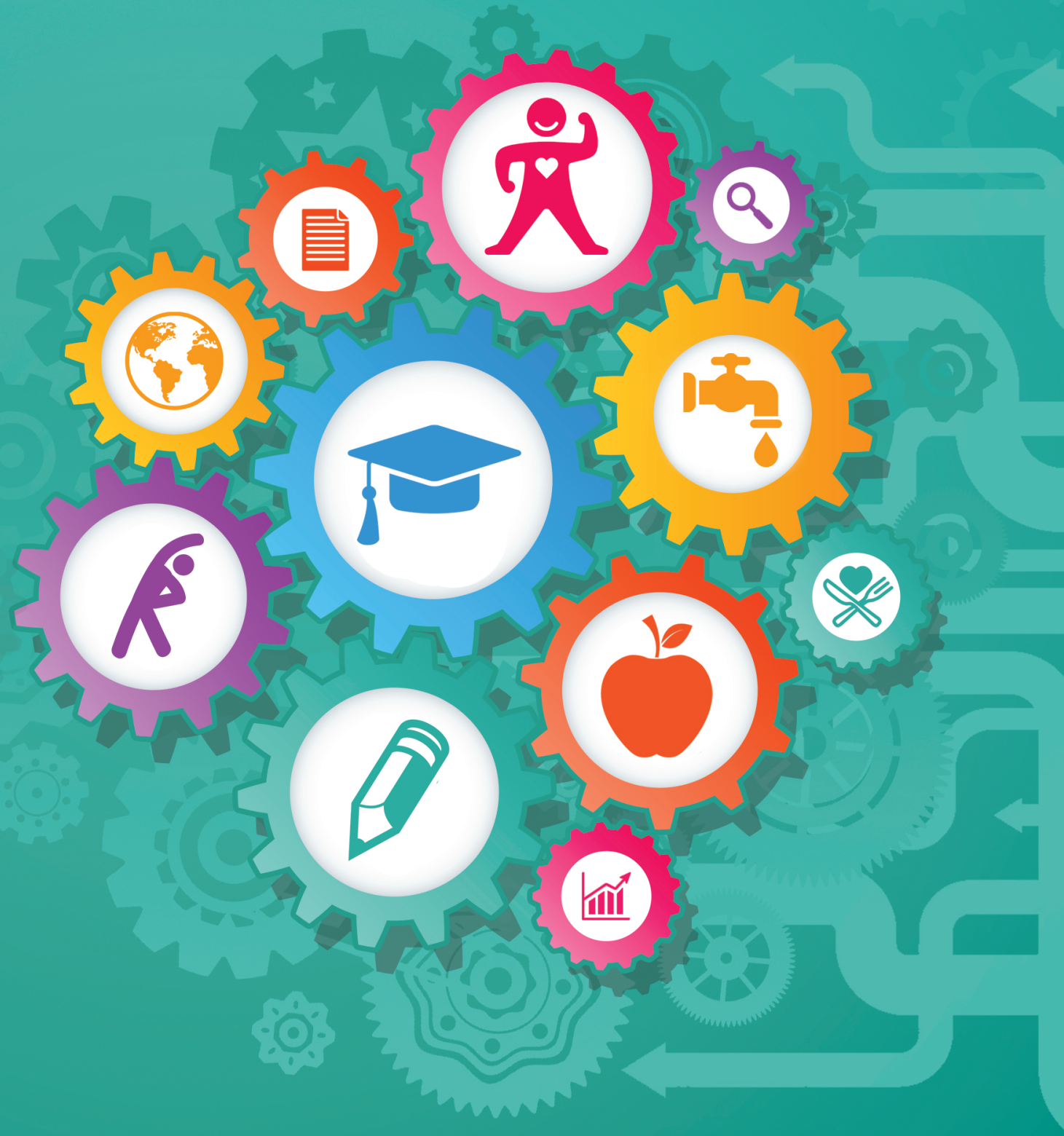

Nina Bartelink 


\section{Evaluating health promotion in complex adaptive school systems: \\ The Healthy Primary School of the Future}

Nina Bartelink 
(C) copyright Nina Bartelink, Maastricht 2019

Printing: ProefschriftMaken || www.proefschriftmaken.nl

ISBN 9789463805322

All rights reserved. No part of this publication may be reproduced, stored in a retrieval system or transmitted, in any form or by any means, electronic, mechanical, photocopying, recording or otherwise, without prior permission of the author or the copyright-owning journals for previous published chapters. 


\title{
Evaluating health promotion in complex adaptive school systems: The Healthy Primary School of the Future
}

\author{
Proefschrift \\ ter verkrijging van de graad van doctor aan de Universiteit Maastricht, \\ op gezag van de Rector Magnificus, Prof. dr. Rianne M. Letschert, \\ volgens het besluit van het College van Decanen, \\ in het openbaar te verdedigen \\ op woensdag 30 oktober 2019 om 10:00 uur
}

door

Nina Henrica Maria Bartelink

18 augustus 1988, Stein 


\section{Promotores}

Prof. dr. Maria Jansen

Prof. dr. Stef Kremers

Prof. dr. Hans Savelberg

\section{Copromoteres}

Dr. Patricia van Assema

\section{Beoordelingscommissie}

Prof. dr. Nanne de Vries (voorzitter)

Dr. Kathelijne Bessems

Prof. dr. Manuela Joore

Dr. Judith de Meij (GGD Amsterdam)

Prof. dr. Gerard Molleman (GGD Gelderland-Zuid, Radboudumc) 


\section{Table of contents}

Chapter 1 General introduction

Chapter 2 The Healthy Primary School of the Future: A contextual actionoriented research approach

Chapter 3 Process evaluation of the Healthy Primary School of the Future:

The key learning points

Chapter 4 Can the Healthy Primary School of the Future offer perspective in the on-going obesity epidemic in young children? - A Dutch quasiexperimental study

Chapter 5 One-and two-year effects of the Healthy Primary School of the

Future on children's dietary and physical activity behaviours: A quasi-experimental study

Chapter 6 Unravelling the effects of the Healthy Primary School of the

Future: For whom and where is it effective?

Chapter 7 The moderating role of the school context on the effects of the Healthy Primary School of the Future

Chapter 8 General discussion

Valorization Addendum

References

Summary

Samenvatting

Curriculum Vitae

Publication list

Dankwoord 



\section{Chapter 1}

General introduction

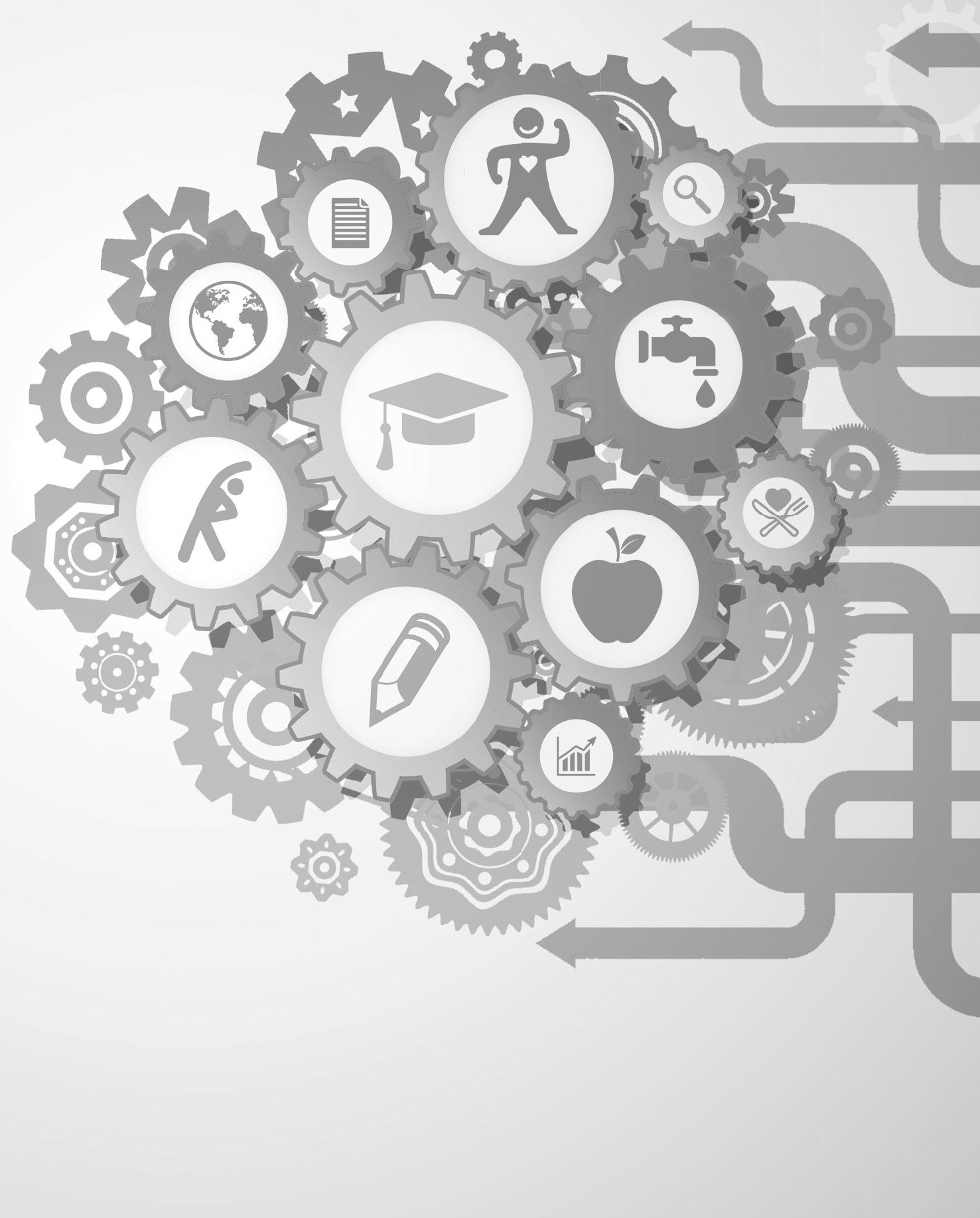



The aim of the research presented in this dissertation was to evaluate the Healthy Primary School of the Future (HPSF) in four complex adaptive school systems. The included studies investigated how HPSF was implemented in the schools, to what extent HPSF had positively contributed to improved health and health behaviours of children, and where, for whom and in which context it was most effective. This introductory chapter presents the overall rationale, the content of HPSF, and the theoretical perspective and principles that guided the research. This leads to the presentation of the overall aim and the main research questions of this dissertation. It ends with an outline of the different chapters.

\section{Background}

\section{Stopping the vicious circle}

Unhealthy habits formed at a young age, such as unhealthy dietary behaviours and low levels of PA, can already in childhood lead to health problems, such as overweight and obesity $[1,2]$. The health behaviours of primary school aged children are suboptimal in many countries, including the Netherlands: only $42 \%$ of children (aged 4-9) consume at least the recommended 150 grams of fruit per day, this percentage drops to $20 \%$ for 9 12 year olds. Prevalence figures of vegetable intake show similar percentages: $41 \%$ of 4 9 year olds and $25 \%$ of $9-12$ year olds eat at least the recommended 150 grams of vegetables per day [3]. Regarding PA, only half (48\%) of Dutch children (aged 4-12) meet the guidelines for PA of 60 minutes of moderate-to-vigorous physical activity (MVPA) per day [4]. The prevalence of childhood overweight and obesity in the Netherlands shows that 13-15\% of Dutch children (aged 2-21) are overweight, and $1.8-2.2 \%$ are obese, which is a 2- to 3-fold increase compared to 1980 [5]. Childhood overweight often tracks into adulthood [6] and is related to reduced quality of life, health problems such as type 2 diabetes, cardiovascular diseases and psychological problems (e.g., low self-esteem), and high health care costs [7-9]. The problem of childhood overweight is even larger, as studies have shown that its prevalence is higher among children with a low socioeconomic status (SES) [10]. An association seems to exist between health status and educational achievement, with health status affecting the capacity to learn, and educational achievements affecting health status [11]. This link between health and education has been indicated as an explanation for the socioeconomic health inequity problems that continue to exist from generation to generation $[12,13]$. Promoting healthy behaviours at an early age may help to improve children's health as well as their educational achievements; both may lead to improved health in later life and disrupt the vicious circle of socioeconomic health inequities. 


\section{School as a setting for health promotion}

Schools can play an important role in promoting healthy behaviours in children, since a significant proportion of a child's day is spent there and schools reach all children from a variety of socioeconomic and ethnic backgrounds [14, 15]. Furthermore, school is one of a diversity of microsystems which interact to shape child development and wellbeing: the impact of changes in the school may also interact with the child's behaviour in other microsystems, e.g., home setting and neighbourhood, which could enhance the effects of health promotion in school $[16,17]$. Moreover, since healthy behaviours also improve academic achievements, school health promotion can contribute to achieve a school's primary educational goals [18]. However, education and health are not often combined in school systems. Worldwide, school health promotion has long been characterized by relatively low priority, fragmentation, and a lack of coordination, partly due to the absence of a legal obligation [19]. This created a situation in which all kinds of external organizations developed health-promoting (HP) interventions to improve a specific aspect of a child's health and well-being [11]. It was hereby assumed that an intervention only impacts a small part of the school and will produce predicted effects in each school context. The primary focus of these interventions was often on increasing knowledge through classroom-based health education and resulted in limited integration of the interventions as implementation stopped after the last lesson. As a result of this situation, schools were overloaded with externally developed HP interventions. Teachers perceived themselves as a 'dumping ground' for all these interventions and complained about the high workload as all these well-intended interventions were added to their regular work [11]. Studies that investigated the implementation and effects of these interventions recommended to use a whole-school approach and to involve the people in the school when developing HP interventions [20]. This should create more ownership for the HP changes and the changes can in this way be better adapted to the school's context.

\section{The Health Promoting School framework: global, European, and national developments}

The Health Promoting School (HPS) framework was developed by the World Health Organization (WHO) in the late 1980s and aims for a whole-school approach to promote children's health and well-being [21]. The development was inspired by the Ottawa Charter, which states that health promotion is a process of enabling people, meaning that people can actively acquire competencies to create more control over their own health and over their environment [22]. The HPS framework uses a broad understanding of health, which means that HPS not only focuses on one specific aspect of health, such as children's BMI, but on improving all aspects of their health, which includes also their mental and social well-being. Moreover, the HPS framework aims for a holistic approach, with a focus on reorienting school systems towards sustainable health promotion [21]. 
This means that HPS not only focuses on classroom-based health education, but also on changes in school policy and the school's physical and social environment, using bottomup involvement of children, parents, and teachers (Figure 1).

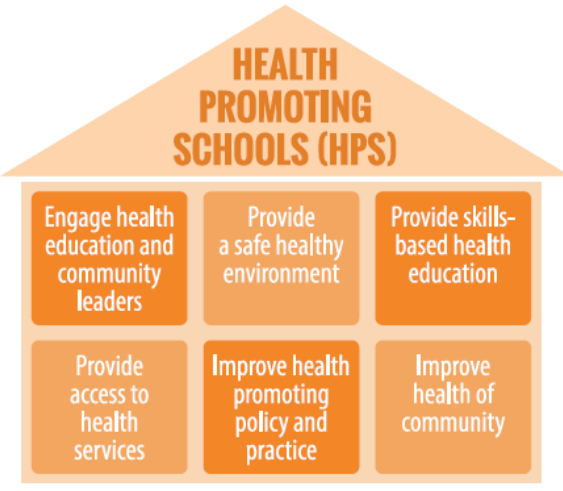

Figure 1. Key features of Health Promoting Schools, derived from WHO [23]

In addition to the development of the HPS framework, the WHO - in collaboration with the Council of Europe and the European Commission - initiated the Schools for Health in Europe Network Foundation in 1992 (SHE, www.schoolsforhealth.org) to stimulate and support school health promotion in Europe [24]. SHE is a non-profit foundation including national coordinators, who are representatives from 33 countries. The national coordinators embrace the HPS framework and have a main role in supporting school health promotion in their countries through contact and dialogue with school authorities, schools, and health promoters. The SHE Research group is a consortium of researchers who provide SHE with a direct connection to a range of universities throughout Europe. In the European region, SHE has developed into an important platform for implementing, supporting, and evaluating health promoting schools.

Similar to many other countries, school health promotion has in the Netherlands long been characterized by relatively low priority, fragmentation, and a lack of coordination [25]. The primary school system in the Netherlands is mainly driven by educational requirements for math, language, reading, and world orientation, which are the statutory performance indicators that are determined at a national level [25]. The responsibility for school health promotion was delegated to the local authorities and did not address these educational requirements [25]. The development of the HPS framework by $\mathrm{WHO}$ has led to innovative initiatives in the Netherlands, such as SchoolBeat ('SchoolSlag') [26-28] and Dutch involvement in European developments regarding school health promotion. All these and other developments throughout the years in which the HPS framework was embraced, have contributed to the development of the Healthy School Programme ('Programma Gezonde School'). This programme aims to integrate health promotion in the DNA of every school in the Netherlands and is 
funded by the Dutch government for the period 2017-2020 [29]. The programme is coordinated by the National Institute for Public Health and the Environment (RIVM), the Association of Public Health Services (GGD GHOR Nederland), and the primary, secondary, and secondary vocational education councils (PO-, VO- en MBO-raad). Within this programme, around forty national organizations are cooperating to promote a healthy lifestyle in schools. The programme is based on four pillars (education, environment, signalling, and policy) to create school health promotion in an integrated way. Schools can apply for financial support at the Healthy School Programme and school health promotion advisors from regional Public Health Services can support schools to implement HP changes that focus on all the four pillars. When schools succeed in implementing HP changes on all pillars, they can apply for a School for Health certificate on one or more of the eight health themes: 1) nutrition, 2) exercise and sport, 3) preventing smoking, alcohol use, and drug use, 4) well-being, relationships, and sexuality, 5) hygiene, skin, and teeth, 6) indoor environment, natural environment, and physical safety, 7) media literacy, and 8) hearing loss.

\section{The effectiveness of HPS}

Several reviews have been published on the effectiveness of HPS in improving the health and well-being of schoolchildren. Overall, school initiatives that were inspired by the HPS framework showed some promising effects, such as improved health behaviours of children, a decline of children's BMI, and improved aspects of mental and social wellbeing [30, 31]. However, these findings were not uniform across the included studies: achieving successful implementation and sustaining the positive health benefits has proven to be challenging [32]. Successfully implementing the HPS framework in schools requires change in both the people in the school as well as the school system itself. This system-wide change has been considered as a complex task to fulfil and actually very little is known yet about how to translate the HPS framework successfully into practice [11]. It has resulted in a considerable gap between the vision of HPS and its implementation [20,32]. This dissertation aims to contribute to a better understanding of the complexity of implementing health promotion in the whole school system. 


\title{
The Dutch primary school system and regional issues
}

\author{
The Dutch primary school system
}

In the Netherlands, primary school consists of study years one to eight, which includes children from age 4 to 12. Attending school is mandatory from the age of five. In 2018, 1.5 million children were enrolled in 6739 primary schools in the Netherlands [33]. A typical Dutch primary school day lasts from approximately $8.30 \mathrm{am}$ to $3.00 \mathrm{pm}$ on Monday to Friday, except for Wednesday, when children finish school around 12.30pm. Schools have a morning break of about 15 minutes when children go outside to play and can eat their own morning snack brought from home. Lunch break time varies between 30-60 minutes: 15 minutes' lunch, when they can eat their own sandwiches brought from home as generally no meals are offered, and 15-45 minutes of free play outside after lunch. In some schools, children can go home during this break to have lunch at home. Approximately 60-120 min/week is spent on physical education classes. Some schools have a sports hall on-site, in other schools children have to commute to the sports hall by foot, bike or bus.

\section{Parkstad: A Dutch region with persistent issues in health and participation}

The Parkstad region is located in the province of Limburg, which is situated in the very south-eastern part of the Netherlands. It consists of eight municipalities and has a population of 250,000 inhabitants (211 square kilometres). The region is especially known as a former mining area that flourished in the first half of the 20th century. Due to the closure of the mines, which started in 1965, the region was faced with severe unemployment among former, often low-skilled, miners and related workforce. For decades after the closure, unemployment rates were higher than anywhere else in Limburg or the Netherlands [34]. Due to the poor economic state of the region, Parkstad also had to deal with social demographic imbalances: young and highly educated people tended to move out of the region, while elderly and less well-educated people stayed [35]. The region is nowadays characterized by a low SES, high prevalence of overweight/obesity, high school dropout rates, and reduced labour participation compared to the mean for the Netherlands [36-38]. These socioeconomic, participation, health, and educational issues are a persistent problem in the region, which continues to exist from generation to generation $[39,40]$.

\section{The Healthy Primary School of the Future}

The socioeconomic, participation, health, and educational issues in the Parkstad region has induced the local educational board 'Movare', situated in the Parkstad region, to take action. They want to compensate for the shortcomings in the development of 
children regarding a healthy lifestyle and to contribute to an optimal development of children's talents. Together with the regional Public Health Services and Maastricht University, they developed the Healthy Primary School of the Future (HPSF). HPSF is based on the HPS framework and aims to sustainably integrate health and well-being within the whole school system, which should lead to a healthier future generation. HPSF intends to establish a co-creation movement in schools by including top-down and bottom-up processes to develop and implement HP changes in all aspects of the school system. On top of the HPS framework, the aim of HPSF is to create some form of positive disruption in the schools by initiating two HP changes top-down: 1) a free healthy lunch each day and 2) daily structured PA and cultural sessions after lunch. The two changes are contextualized bottom-up and should lead to momentum for more bottom-up processes to implement additional HP changes in the school. The choice for the two top-down HP changes led, especially in the beginning, to a focus on diet, PA and body weight in the HPSF initiative, despite the aim for a holistic approach to improve all aspects of children's health, including their mental and social well-being.

\section{Four participating schools}

In March 2013, 12 out of 53 schools governed by the Movare educational board were informed about the initiative. Four schools (S1-4) gave their initial consent and spent a whole school year (2014/2015) creating bottom-up support. Due to differences in this bottom-up support, HPSF was split into two versions: 1) implementation of both the lunch and the structured PA and cultural sessions, and 2) implementation of the structured PA and cultural sessions only. S1 and S2 continued with the first version of HPSF, in this dissertation referred to as 'the full HPSF'. To realize these changes during the lunch break, both schools extended the lunch break time and their school day. S3 continued with the second version. The fourth school dropped out because of a lack of bottom-up support and a new ' 54 ' was included at the end of the school year, which also continued with the second version of HPSF. S3 and S4 are in this dissertation referred to as 'the partial HPSF', and did not extend their lunch break time or school day. All four schools started implementation in November 2015. Even though the schools were situated in the same region in the Netherlands and were part of the same educational board, each school had their own specific context (Table 1). This specific context of each school resulted in each school implementing their own contextualized version of HPSF. 
Table 1. The four participating schools prior to the start of HPSF in 2015

\begin{tabular}{|c|c|c|c|c|}
\hline & School 1 & School 2 & School 3 & School 4 \\
\hline School population & $\begin{array}{l}324 \text { children } \\
26 \text { teachers }\end{array}$ & $\begin{array}{l}234 \text { children } \\
15 \text { teachers }\end{array}$ & $\begin{array}{l}233 \text { children } \\
16 \text { teachers }\end{array}$ & $\begin{array}{l}389 \text { children } \\
21 \text { teachers }\end{array}$ \\
\hline Municipality & Landgraaf & Landgraaf & Brunssum & Landgraaf \\
\hline Start school day & $8: 30$ & $8: 30$ & $8: 30$ & $8: 30$ \\
\hline End school day & $15: 00$ & $14: 45$ & $14: 45$ & $15: 00$ \\
\hline Lunch break time & $45 \mathrm{~min}$ & $30 \mathrm{~min}$ & $45 \mathrm{~min}$ & $60 \mathrm{~min}$ \\
\hline Organizational issues & $\begin{array}{l}\text { - In the middle of a } \\
\text { merging process; } \\
\text { - New school building }\end{array}$ & $\begin{array}{l}\text { - Planning a merging } \\
\text { process; } \\
\text { - Renovation of } \\
\text { school building and } \\
\text { temporary location }\end{array}$ & - Staff turn over & $\begin{array}{l}\text { - Last minute } \\
\text { participation in } \\
\text { HPSF }\end{array}$ \\
\hline
\end{tabular}

\section{Coordination and organization of HPSF}

Each school selected a teacher as school coordinator, who managed HPSF in their school. Overarching the four schools, the HPSF initiative was led by a project leader from Movare and an executive board with representatives of Movare, the regional Public Health Services and Maastricht University, including the project leader. These organizations, together with several other external partners, cooperated in HPSF to support the schools in the development and implementation of the HP changes. The two top-down initiated changes, i.e., providing a lunch each day and daily structured PA and cultural sessions, were implemented by external pedagogical employees (PE) provided by childcare organizations. A PE coordinator per school acted as the contact person for all external PE in that school. The lunch products were provided by catering services (Sodexo). The instructions for the PA sessions were provided by a sports and leisure organization (the Move factory). The Move Factory also supported the external PE during implementation when needed, and after a year they provided a training course ( 8 sessions of 2 hours each) to supply them with additional tools in how to motivate children to participate actively during the PA sessions. A health promoter from the regional Public Health Services was assigned to each school to provide support when needed. Researchers from Maastricht University monitored and fed back results to the schools to support the process of change. The provincial authorities supported the initiative financially. Regular meetings were held at the school level and overarching the four schools to keep each other updated, to provide feedback, and to discuss the process of change. Each school initiated regular meetings between the school coordinator and PE coordinator, working groups with teachers and parents, and children's voice groups. The health promoters of the four schools met regularly to keep each other updated on the on-going processes in each school. A project team was created with representatives of all partners involved: the four schools, Movare, regional Public Health Services, Maastricht University, the Limburg provincial authorities, childcare organizations, the caterer, and sports and leisure organizations. 


\section{Theoretical perspective and principles}

\section{Complex adaptive school systems}

The previous experiences in school health promotion have led to the decision to adopt a new perspective that deals with the complex and adaptive nature of schools: a complex adaptive systems perspective. A complex adaptive system can be described as a system that consists of many interacting components and has the capability to selforganize and adapt. The system's behaviour is typically non-linear, not easily controlled or predicted, and tends to self-organize to a state of stability [32, 41-45]. Embracing this perspective to schools determined the choices regarding HPSF and the overall research approach. It implies the introduction of some new concepts that do not yet have one clear definition. Therefore, an overview is provided of how the different concepts are interpreted in the current dissertation (Table 2).

Table 2. Explanation of concepts related to complex adaptive school systems

\begin{tabular}{|c|c|}
\hline Concept & Explanation \\
\hline $\begin{array}{l}\text { Complex adaptive } \\
\text { system }\end{array}$ & $\begin{array}{l}\text { A system that consists of many interacting components and has the capability to self- } \\
\text { organize and adapt. The system's behaviour is typically non-linear, not easily controlled or } \\
\text { predicted, and tends to self-organize to a state of stability. }\end{array}$ \\
\hline School context & $\begin{array}{l}\text { The specific circumstances and characteristics of a school, which relates to the social, } \\
\text { political, economic, and physical environment; the characteristics, behaviours, wishes, } \\
\text { and needs of the people in the school; the wider community in which the school is } \\
\text { located; as well as the history and organization of the school. }\end{array}$ \\
\hline Setting & $\begin{array}{l}\text { A place or social context in which people engage in daily activities, in which } \\
\text { environmental, organizational and personal factors interact. Examples of settings are } \\
\text { home, school, workplace, prisons, hospitals, and communities. }\end{array}$ \\
\hline Systems dynamics & $\begin{array}{l}\text { The complex behaviours of organizational and social systems that are the result of } \\
\text { continuous interactions between components in the system and both balancing and } \\
\text { reinforcing feedback loops that develop between these interacting components. }\end{array}$ \\
\hline Disruption & $\begin{array}{l}\text { Something significant that happens, preventing the system or processes in the system to } \\
\text { continue as usual; it causes the future trajectory of the system's dynamics to change. }\end{array}$ \\
\hline
\end{tabular}

The school is thus a setting that can be considered as a complex adaptive system, in which each school has its own context. Before going into more detail on schools as complex adaptive systems and how we dealt with this in the reported studies, first more insight is provided regarding the concept of complexity. What is meant by complexity has been explained by other researchers by comparing a complicated and a complex problem $[43,46]$. Sending a rocket to the moon can be considered a complicated problem. It requires great skill and numerous interacting components. However, it can be divided into discrete sets of actions with stable, predictable, and linear consequences. When such a complicated problem is solved, it remains solved, and it can successfully be repeated. In contrast, raising a child can be considered a complex problem. It has an unpredictable and non-linear nature between actions and outcomes. Even though raising 
a child gives experiences, there are no guarantees for success in the future or when raising another child. While a complicated machine such as a rocket is passively acted upon by human actors, children and parents are active agents, whose behaviour continuously adapts in response to feedback from one another, which generates behavioural patterns for the whole family. These behaviours at home are not isolated and interact with other systems the child or parent is part of, such as the school system.

To illustrate the complex adaptive school system, key characteristics are described and applied to schools [11, 47-50]. These characteristics of complex adaptive systems are closely linked, which makes it impossible to explain them in absolute separation; some overlap among the characteristics is inevitable.

Nested systems structure: Complex adaptive systems are open systems with fuzzy boundaries. Each system is a part of some other system, in which each system can be a sub-system in a bigger system, and/or a supra system for a smaller system. People in school belong to and are influenced by many systems simultaneously. Change in schools can create change in families and the wider community, but also the other way around. Change in the family or wider community can also influence the school's functioning.

Unpredictability: The overall behaviour of a complex adaptive system cannot be directly predicted from the elements within it, and it is more than just the sum of its parts. Any change in a school can have unexpected factors which can influence the outcomes. This means that in school health promotion there is no guarantee that a HP initiative creates change, that changes will be in the desired direction, or that they will be sustained.

Autonomous agents: A complex adaptive system usually consists of a changing population, referred to as 'agents'. Agents in a school include the children, teachers, parents, and other employees in school. Even though schools have rules which organize the individuals' behaviour and shape the whole school's functioning, individual agents are still, to some degree, autonomous. Agents act in ways that are based on a combination of their knowledge, experience, feedback from the environment, local values and rules. In other words, there are several ways to do things, and agents can make their own choices, which add to the unpredictability of the behaviour of the system.

Self-organization: A complex adaptive system has no centralized control, but is decentralized due to individual autonomous actions: the changes in the system emerge from a process of self-organization rather than being controlled externally or by a centralized body. Schools are controlled by multiple sources such as teachers, children, parents, education authorities, community, media, and politics. One agent can already change the context, which can create change in other agents. These changes can reshape the system's collective behaviour. 
Interaction: In a complex adaptive system interactions exist continuously. An interaction is a two-way process in which elements, systems, and/or agents respond and adapt to each other. A variety of interactions in schools exist, e.g., among children, teachers and other people in the school, but also between physical and social elements in the school context, or between the school and the wider community.

Adaptation: What emerges in a complex adaptive system can be interpreted as a function of on-going adaptations that may continually lead to new needs, interests and opportunities. Adaptation produces impacts on other elements in the system as they are interconnected and it aims to create solutions to make changes sustainable. To have sustainable health promoting schools, flexibility is needed so that schools can adapt to the changing conditions.

Non-linearity: Complex adaptive systems have non-linear behaviour, meaning they may respond in different ways to the same input depending on their context. Large HP efforts in a school can lead to no impact on the school system, whereas small efforts can produce large impact at a so-called bifurcation or 'tipping' point. This tipping-point indicates the momentum in the school, in which it shifts from slow and gradual acceptance of changes to fast and widespread acceptance. It is hard to predict when this tipping point is reached. As a consequence, no guarantees exist whether HP changes in a school will have an impact on the system and whether it will lead to the expected outcomes.

Feedback loops: Feedback loops are a circular process in which a system's output is returned or 'fed back' into the system as input. Two kinds of feedback exist: reinforcing (or positive) and balancing (or negative). Reinforcing feedback accelerates a change away from a starting point whereas balancing feedback slows down or corrects a change in a system that is moving away from the starting point. Changes in a school give rise to these two different feedback loops, which may reinforce the implementation of the changes or lead to discontinuance of the changes. Feedback might be internal, from people or components in the school, or external, from families or other aspects in the wider community.

\section{A contextual action-oriented research approach (CARA)}

Considering schools as complex adaptive systems implies a need for more contextspecific thinking to integrate health promotion in schools $[47,48,51]$. Consequently, to evaluate change in such a complex adaptive system, the evaluation methods need to be sensitive to the dynamics in the local context $[49,52,53]$. To find a way to adapt our research of HPSF to this complexity and to deal with the differences between the four school contexts, we translated the principles of action research into a contextual actionoriented research approach (CARA). Through the use of monitoring and feedback, CARA aims to identify where changes are interacting with contextual aspects of the school, 
not only to evaluate the process of changes, but also to support the schools in this process. CARA is explained in one of the chapters in this dissertation (Chapter 2), which illustrates in detail how we dealt with evaluating changes in complex adaptive school systems.

\section{Programme theory}

Based on the complex adaptive systems perspective, we developed a programme theory (Figure 2). This programme theory visualizes the hypothesized process of how HPSF integrates into the school context. It acts as a conceptual basis for the main research questions, which the included studies aim to answer. Resulting from the perspective that is embraced, several key assumptions are part of this programme theory. First, it is assumed that during the process of change, the school system tries to find a new balance: it tends to self-organize to a state of stability, either by pushing the change out of the school or by integrating the change into the school [43]. A second key assumption concerns non-linearity in the cause-effect relation: it is assumed that small changes in a school can produce large effects at a so-called 'tipping' point. A third assumption is that realized changes may shift the school's norms toward a focus on health and well-being, thereby creating momentum for additional HP changes [43]. This third assumption is visualized in the loop in the bottom of the programme theory. Finally, a fourth assumption is that even when a change is similar, the school context will determine its impact. This moderator-effect of the context is visualized in the top right of the model.

To fully understand the content of the programme theory, one can best start on the left side of the model, which shows the input. This input can be seen as an 'event' that attempts to positively disrupt the pre-existing dynamics in the school context in order to integrate health promotion $[43,54]$. This school context should be understood as thoroughly as possible, because it determines the starting point of HPSF. After the introduction of HPSF into the school context, the process of development, implementation, and integration of HP changes develops in the school. During this process it is hypothesized that HPSF will continuously interact with the school context: on the one hand, the school context impacts the development and implementation of the HP changes in the school, and on the other hand, the context may respond to the new way of working, which may lead to changes in the school context. Overall, the process of change should lead to the realization of HP changes that fit the school's context. The combination of all these contextualized HP changes should impact children's health behaviours and, through this, their health and well-being. 


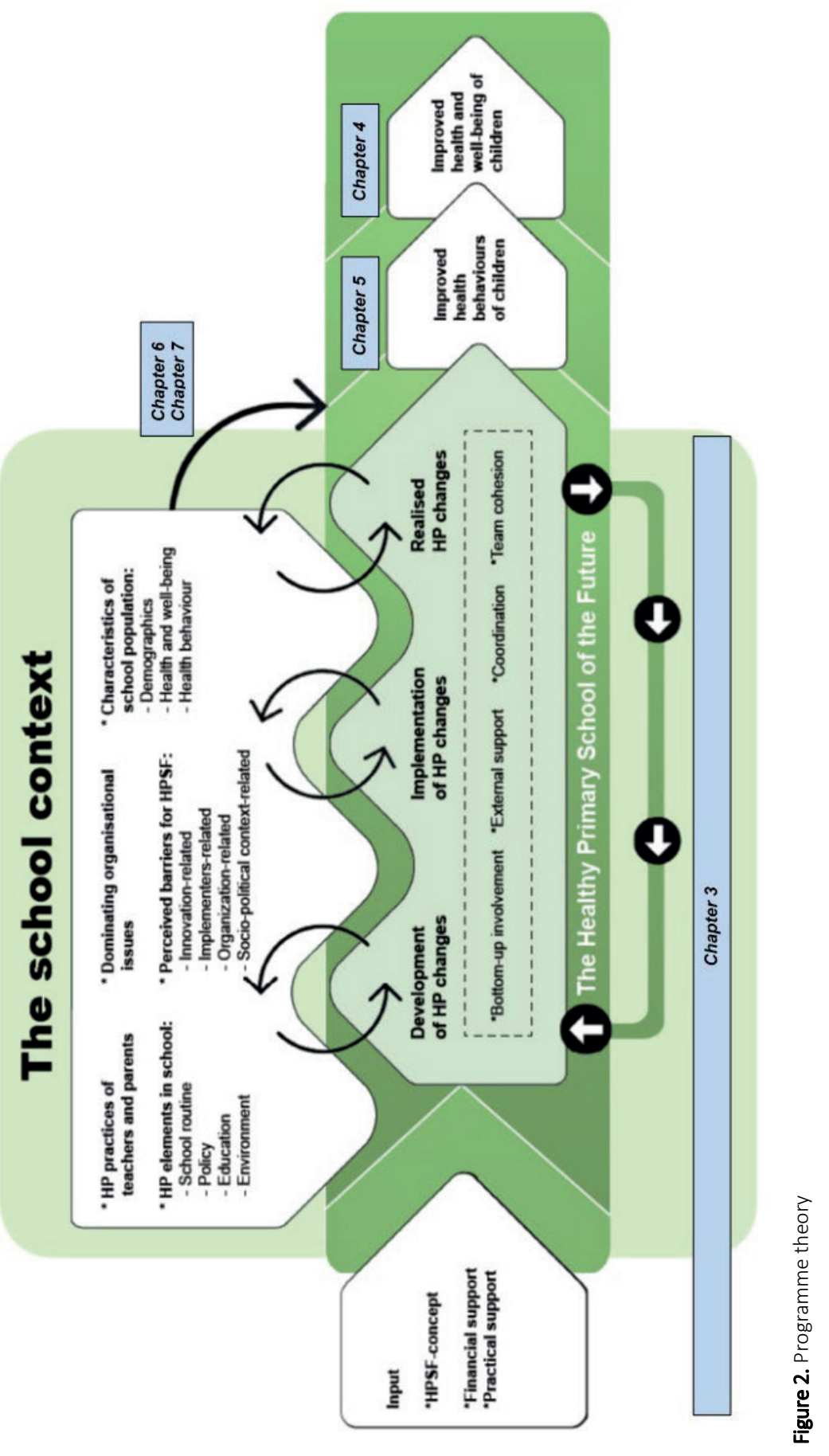




\section{The current dissertation}

\section{Position within the overall study of HPSF}

The current research presented in this dissertation is part of an overall study in which a multidisciplinary research group investigates the impact of HPSF on, among others, children's health and well-being, their educational achievements, its cost-benefits, and the legal consequences of HPSF [55]. This overall study includes four intervention schools and four control schools. Ethical approval (14-N-142) for the overall study was given by the Medical Ethics Committee Zuyderland, located in Heerlen (Parkstad, the Netherlands). Funding for implementation is provided by the Province of Limburg until the end of 2019. The four schools have committed to continued implementation after 2019 and to make the changes sustainable in their school system. Data collection will take place until 2019 to study the effects during four years of exposure. The current dissertation is part of this overall study and focuses on the first two years of implementation of HPSF, in which the primarily focus is on aspects related to healthy nutrition and PA.

\section{The aim and outline of this dissertation}

The aim of the research presented in this dissertation was to evaluate the Healthy Primary School of the Future (HPSF) in four complex adaptive school systems. The included studies investigated how HPSF was implemented in the schools, to what extent HPSF had positively contributed to improved health and health behaviours of children, and where, for whom and in which context it was most effective. Five main research questions were formulated:

1. How was HPSF developed and implemented and how did it interact with the context of the four schools?

2. What was the effect of HPSF on children's BMI z-scores and their dietary and PA behaviours after one and two years?

3. What was the effect of HPSF on children's dietary and PA behaviours at school and at home?

4. To what extent did HPSF have different effects within specific subgroups of children?

5. What was the moderating role of the school context on the effects of HPSF?

Chapter 2 elaborates on the way in which we dealt with examining changes in complex adaptive school systems. It describes the use of CARA and how we, as researchers, were able to contribute to the HPSF initiative and conduct a thorough evaluation at the same time. Chapter 3 describes the process evaluation of HPSF, which relates to the first 
research question. The study uses a mixed-methods design and explores the processes through which HPSF and the school context adapt to one another over time. The next three chapters, chapters 4-6, present the evaluation of the effects of HPSF, which relates to the research questions two to four. The studies use a quasi-experimental design to examine the changes in children's health and health behaviours after one and two years' follow-up and where and for whom HPSF was effective. Chapter 4 examines whether HPSF could offer some perspective in the on-going obesity epidemic. It presents the results of the effect of HPSF on children's BMI z-score and whether HPSF has different effects on this outcome within specific subgroups of children (gender, age, SES, weight status). Chapter 5 studies the overall changes in children's dietary and PA behaviours. Chapter 6 aims to unravel the effects on children's health behaviours as found in Chapter 5. The study investigates the separate effects on children's health behaviours in school and at home and whether HPSF has led to different effects within specific subgroups of children (gender, age, SES, weight status, parental practices). Chapter 7 investigates the moderating role of the school context on the effects of HPSF, which relates to the last research question. The study uses a quasi-experimental design to assess the effects of HPSF in each school and a mixed-methods design to examine the moderating role of the school context. It focuses on several contextual aspects: characteristics of the school population, teacher's HP practices, implementers' perceived barriers, school's HP elements, and dominating organizational issues. Chapter 8 discusses the most important findings from the reported studies. These findings and our experiences are used to reflect on the research approach and the theoretical perspective. Finally, an overall conclusion is provided regarding both the effects and strategies of HPSF as well as the application of CARA. 




\section{Chapter 2}

The Healthy Primary School of the Future:

A contextual action-oriented research approach

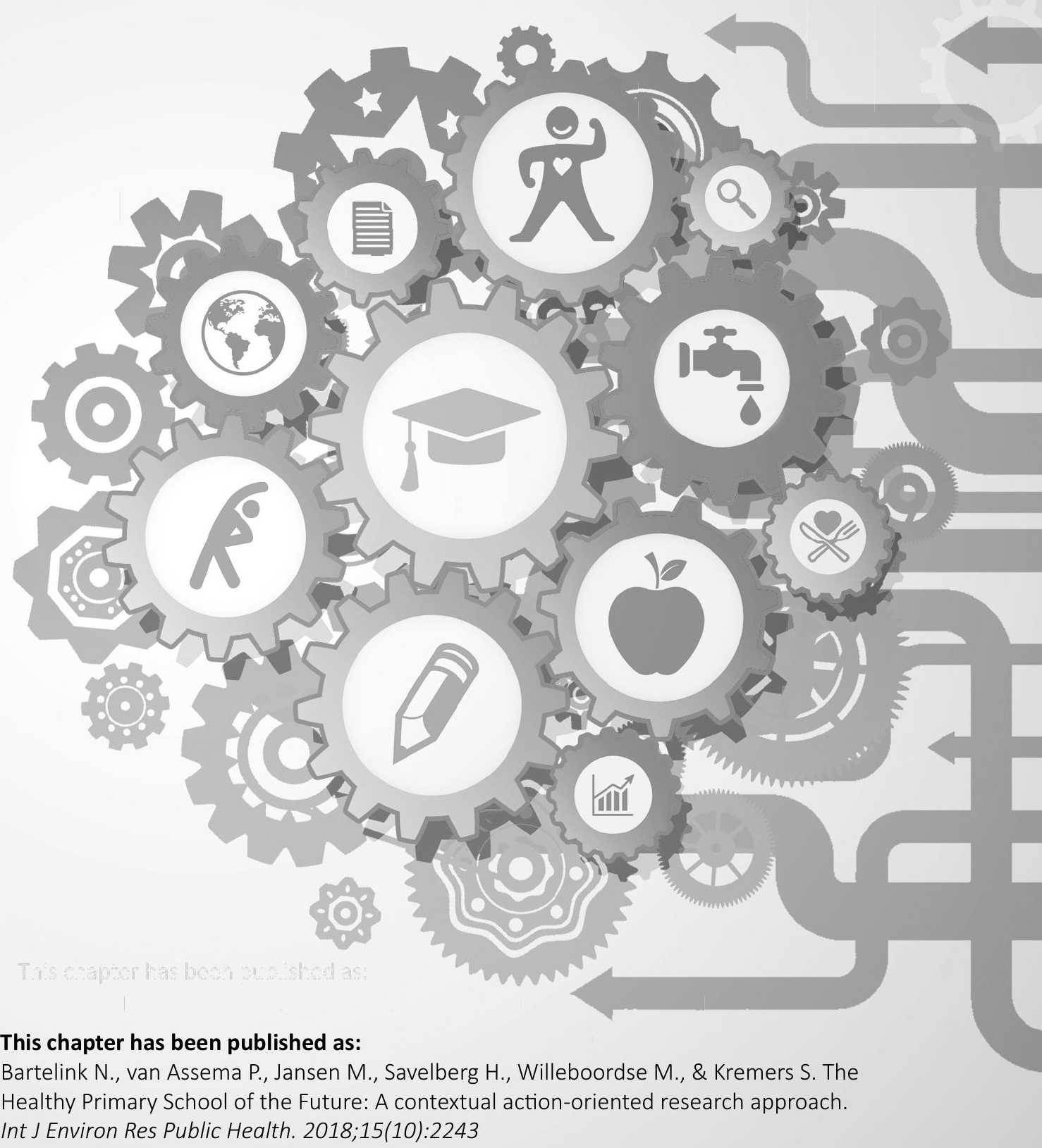




\section{Abstract}

\section{Background}

Schools can play an important role in promoting children's health behaviours. A Dutch initiative, 'The Healthy Primary School of the Future', aims to integrate health and wellbeing into the school system. We use a contextual action-oriented research approach (CARA) to study the implementation process. Properties of CARA are its focus on contextual differences and the use of monitoring and feedback to support and evaluate the process of change. The aim of this article is to describe the use of the approach.

\section{Methods}

Four schools (each with 200-300 children, aged 4-12 years) were included; all located in low socioeconomic status areas in the south of the Netherlands. Data collection methods include interviews, observations, questionnaires, and health and behavioural measurements. Research contributions include giving feedback and providing schools with a range of possibilities for additional changes. The contextual data we examine include schools' health promoting elements, practices of teachers and parents, dominating organizational issues, and characteristics of the student population; process data include the presence of potential barriers to changes.

\section{Discussion}

CARA is an adaptive research approach that generates knowledge and experiences on how to deal with health promotion in complex systems. We think this approach can set an example for research efforts in comparable initiatives. 


\section{Background}

Children living in low socioeconomic status (SES) communities are more likely to have unhealthy behaviours that include physical inactivity and unhealthy dietary habits, compared to those living in high-SES communities $[10,56]$. These unhealthy behaviours can lead to health problems such as overweight/obesity, type 2 diabetes, cardiovascular diseases, and mental health problems, which can develop even at a young age [57]. Promoting healthy behaviours at an early age may help to improve children's health, as well as their educational achievements; both may lead to improved health in later life [58]. Schools can play an important role in this, since they reach all children and form a strong social network of teachers and children who can influence one another, and since a significant proportion of a child's day is spent at school [59]. Moreover, school and home are both part of a child's mesosystem: changes in the school may also influence the home environment, which could enhance the effects of school health promotion $[16,17]$.

Despite the school's potential to help improve children's health, school health promotion in the Netherlands at the beginning of the 21st century was marked by relatively low priority and lack of coordination and fragmentation, and was often supplydriven [25]. Traditionally, the Dutch primary school system (for children aged 4-12 years) has been mainly driven by educational requirements determined at the national level by the Ministry of Education, Culture and Science, which focus on maths, language, reading, and world orientation. Due to these educational requirements, the interest in, and priority given to, the implementation of health promoting (HP) changes at schools is limited, as these changes do not directly address the educational requirements [25]. As a result, HP changes are only coincidentally implemented and often lack systematic coherence and sustainability, as the changes are not embedded in the school system. Whereas educational requirements have been defined at the national level, the Ministry of Health, Welfare and Sports has delegated the responsibility for health promotion to the local authorities, leading to further fragmentation. Local health organizations have to compete for the attention of schools to make them realize the importance of health promotion and its implementation. This leads to a supply-driven approach, which can irritate schools due to the abundance of initiatives offered to them that do not really match their needs [60]. On top of all these issues, the school itself is a complex system, characterized by a large number of interacting institutional elements [48]. This means that it depends on the specific school context how suitable a change towards health promotion is [47]. It also means that each implemented change will have different effects at each school; there is always an interaction between the change and the school context $[47,53,61]$.

This complexity of the school system, together with the contextual differences between them, the fragmentation of school health promotion, and the worrying increase in unhealthy behaviours among school children, have induced the local educational board 'Movare', the Regional Public Health Services (RPHS), and Maastricht University (UM), all 
situated in the southern part of the province of Limburg, to take action. Movare's primary schools are in the former mining area of Parkstad, in which eight municipalities around the city of Heerlen collaborate (211 square kilometres; 250,000 inhabitants). This region is characterized by a low SES, compared to the mean for the Netherlands [36, 37, 55]. Health and educational issues, such as a high prevalence of overweight/obesity and high school dropout rates compared to the national average, are a persistent problem in this region, and continue to exist from generation to generation [39, 40, 55, 62]. Therefore, the three organizations (Movare, RPHS, UM) developed the 'Healthy Primary School of the Future' (HPSF): a Dutch initiative which aims to sustainably integrate health and well-being within the school system [55]. HPSF intends to go beyond traditional temporary and superficial top-down solutions and to establish a co-creation movement in schools towards systematic incorporation of health and well-being. This incorporation ideally leads to sustained changes that become embedded in the DNA of the school. In other words, HPSF aims to add-in, instead of adding-on, health and well-being to the school system. The initiative builds upon the principles of the health-promoting school (HPS) framework, which aims to create a healthy school environment using a school system approach. HPS focuses not only on classroom-based health education, but also on changes in school policy and the schools' physical and social environment [63], using bottom-up involvement of pupils, parents, teachers, and staff.

Co-creation processes within the school system are challenging for researchers to study in a scientifically sound manner. Traditionally, many action researchers in school interventions have followed the cycle of needs assessment, development, implementation, monitoring, and evaluation of change $[64,65]$. However, the limitation of following these steps is that they suggest a logical, causal process, which is difficult to identify in a complex school system initiative [66], where changes are interacting with each other and with other contextual aspects of the school $[49,53]$. Therefore, we felt the need to find a way to adapt our research to this complexity. As this study is not the first to deal with initiatives in complex systems, we have been inspired by the existing literature, from which various considerations and insights were gathered [47-49, 67, 68]. These insights into systems thinking have led us to adapt the principles of action research into a contextual action-oriented research approach (CARA). The purpose of CARA is to contribute as researchers to the process of a complex intervention initiative, and to conduct a thorough evaluation of the process and its final outcomes that addresses the importance of the implementation context. Basic properties of CARA are its specific focus on contextual differences, and the use of monitoring and feedback to both support and evaluate the process of change. The approach centers around four key questions: (1) What is the pre-existing context of each school?; (2) How does the process of change in each school evolve and which factors affect this process?; (3) How can research contribute to the process of change?; and (4) Do children's health and health behaviours improve as a result of the HP changes? 
The aim of this article is to elaborate on the way in which we are dealing with the complexity of the school system and the HPSF initiative by using CARA and how we are able to contribute to the initiative and at the same time conduct a thorough evaluation. HPSF has a broad focus on different aspects of health and well-being. The present study focuses on two key aspects, i.e., healthy nutrition and physical activity (PA).

\section{Methods}

\section{The Healthy Primary School of the Future}

Four schools, each with 200-300 children (aged 4-12 years) and with 15-30 teachers per school, are included in the HPSF initiative (whose implementation started in November 2015) as pilot schools. In addition, four comparable control schools in the same region were also included. Information about the recruitment of these eight schools has been described by Willeboordse et al. [55]. The three cooperating organizations, Movare, RPHS, and UM, have introduced two top-down changes to the schools' system: 1) providing a free healthy lunch each day; and 2) a full hour of structured PA each day, both prepared and led by external pedagogical staff, provided by childcare organizations [55]. While in other national school systems these may represent usual practice, these changes are hypothesized as disruptive to the Dutch school system, because the provision of school lunches and structured PA sessions are not usual practice in Dutch schools. The schools involved teachers and parents in the one-year decision and development process to adapt the two changes to their context. The schools only decided to start implementation of the two changes if they had the teachers' support and at least $80 \%$ parental support. It is expected that the changes will create increased interest of the school in healthy nutrition and PA. In addition, it is assumed that they will create momentum for additional HP changes fitting the context of each school. As part of the HPSF initiative, one teacher in each school is appointed as school coordinator. She or he develops each change with working groups of parents, teachers, and children, as well as two closely involved employees of the RPHS (a youth nurse and a health promoter) who are assigned to each school to provide support when needed. In addition, a project team was created, including the schools, Movare school board, UM, childcare organizations, catering services, sports and leisure organizations, RPHS, and the Limburg provincial authorities. The provincial authorities will continue to support the project financially until 2019 to realize a breakthrough in the worrying health status of the young generation. 


\section{Contextual action-oriented research approach (CARA)}

The aim of research into the HPSF initiative is not only to evaluate, but also to support the process of change in the schools, with a specific focus on the contextual differences. To be able to achieve this, we used CARA, which builds on our previous experiences in school health promotion and on the international literature regarding new insights into complex systems thinking $[47,48,53,61]$. CARA is an adaptation of action research principles, whereby the traditional linear steps are let go as they suggest a logical, causal process. In contrast, CARA aims to identify where changes are interacting with contextual aspects of the school $[49,53]$. Table 1 shows how the traditional steps of action research (column 1 ) are combined with the insights of complex systems thinking (column 2) to form CARA. The table also presents the methods, based on the four key questions (column 3). For each key question, the relevant insights and methods are discussed in more detail below.

Table 1. Considerations and insights on initiatives in complex systems

\begin{tabular}{|c|c|c|}
\hline \multirow{2}{*}{$\begin{array}{l}\text { Action research } \\
\text { The traditional } \\
\text { steps }\end{array}$} & \multicolumn{2}{|c|}{ Contextual action-oriented research approach (CARA) } \\
\hline & $\begin{array}{l}\text { Considerations and insights from existing } \\
\text { literature }\end{array}$ & Key questions and methods \\
\hline $\begin{array}{l}\text { Assessment of } \\
\text { needs, interests, } \\
\text { and opportunities }\end{array}$ & $\begin{array}{l}\text { - The school system itself is a complex } \\
\text { system as it is characterized by a large } \\
\text { number of interacting institutional } \\
\text { elements [48]. } \\
\text { - Besides contextual differences between } \\
\text { schools, each implemented change will } \\
\text { also work differently at each school; } \\
\text { there is always an interaction between } \\
\text { intervention and context [47, 53, 61]. } \\
\text { - The willingness to participate in a process } \\
\text { of change depends on motivation, } \\
\text { capacity, and opportunity [69]. }\end{array}$ & $\begin{array}{l}\text { 1. What is the pre-existing context of each } \\
\text { school? } \\
\text { Examining: } \\
\text { Health-promoting (HP) practices of } \\
\text { teachers and parents, HP elements in } \\
\text { school, dominating organizational issues, } \\
\text { innovation-, implementers-, organization, } \\
\text { and socio-political context-related barriers } \\
\text { for HPSF, and characteristics of the } \\
\text { student population. } \\
\text { Methods: } \\
\text { - Interviews }\end{array}$ \\
\hline $\begin{array}{l}\text { Development of } \\
\text { change }\end{array}$ & $\begin{array}{l}\text { - A process of change in a complex system } \\
\text { does not have a linear cause-effect } \\
\text { relationship: e.g., small changes can } \\
\text { produce large effects at so-called }\end{array}$ & $\begin{array}{l}\text { - Barrier questionnaire } \\
\text { - Practices questionnaire } \\
\text { - Health and behavioural measures }\end{array}$ \\
\hline tation of & $\begin{array}{l}\text { 'tipping-points' (non-linearity) }[48,70] \text {. } \\
\text { - How suitable a change is, depends on the } \\
\text { school context }[47,68] \text {. } \\
\text { - A variety of factors can influence the } \\
\text { implementation of a change in a school } \\
\text { setting, such as factors relating to the } \\
\text { implementers, the innovation, the } \\
\text { organization, and the socio-political } \\
\text { context [71]. } \\
\text { - An intervention that is conceived as an } \\
\text { add-in rather than an add-on to existing } \\
\text { school system is more likely to be } \\
\text { implemented and sustained successfully } \\
\text { [72]. } \\
\text { - Implementation of a change will be more }\end{array}$ & $\begin{array}{l}\text { 2. How does the process of change in each } \\
\text { school evolve and which factors affect } \\
\text { this process? } \\
\text { Examining: } \\
\text { The process of change, i.e., the adoption, } \\
\text { implementation, and integration of the HP } \\
\text { changes, and its interaction with the } \\
\text { school context. } \\
\text { Methods: } \\
\text { - Interviews } \\
\text { - Observations } \\
\text { - Barrier questionnaire } \\
\text { - Practices questionnaire }\end{array}$ \\
\hline Monit & - Implementatıon of a change will be more & \\
\hline
\end{tabular}


in) each context

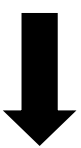

Evaluation successful and will lead to greater ownership and commitment if it involves a process of mutual adaptation, where a change is modified to suit the needs, interests and opportunities of the school, and where the people in school are open to (major) adjustments and will adapt to meet the requirements of the change [73].

- Bottom-up approach is needed as teachers, children, and their parents know best which changes are most appropriate for their school, and this approach will create greater ownership of the changes [70, 74].

- Top-down approach is needed as the external experts involved have specific health promotion knowledge, skills and experiences, which may lead to more effective changes [70, 74].

- Monitoring and evaluation is no longer merely an external observation of strategies to implement changes, but becomes one of the strategies itself [48].

- The attitude of the researchers is no longer neutral and fully objective, but involves joining in discussions and giving support to the innovators whenever possible from their specific knowledge, skills, and experiences [48].

- Regular feedback provides valuable guidance to the process of change in the schools [75].
3. How can research contribute to the process of change?

Examining:

Supportive contributions of the researchers to the schools.

Contributions offered:

- Providing feedback

- Defining possible behavioural goals

- Offering a range of possibilities ('fruit basket model') to show all possible additional changes, while leaving the decision to the schools.

Methods:

- Interviews

4. Do children's health and health behaviours improve as a result of the HP changes?

Examining:

How, for whom and in what circumstances does HPSF affect children's health and health behaviours?

Methods:

- Health and behavioural measures

- Moderator analyses and qualitative comparison to combine the data of the effect study with implementation and contextual data.

\section{What is the pre-existing context of each school?}

This first key question regards examining the school context to determine the starting point of HPSF. Each school context is part of a complex system [48]. Therefore, introducing fundamental changes to a system first requires an understanding of this context [68]. Thus, the first phase involves the investigation of each school context. Each school has decided autonomously whether to participate in HPSF. Existing literature shows that in this decision and the further process of change, several contextual factors might be of direct importance, e.g., HP practices of teachers and parents; HP elements in the school (school routine, policy, education, and environment); dominating organizational issues (e.g., staff turnover); innovation-, implementers-, organization-, and socio-political context-related barriers for HPSF perceived by implementers; and characteristics of the student population (health, well-being, health behaviours, demographics) $[69,71]$. To assess the school context, we use mixed methods that are appropriate to obtain rich information, and that can rapidly be translated into real-time 
feedback for the schools. For all methods, we use a framework of possible behavioural goals regarding healthy nutrition and PA (Table 2). These goals were defined by the research team during the preparation year, by applying insights from the PrecedeProceed model about ways to define clear behavioural goals [76]. In addition to their use as a framework for the researchers, the goals may also work as an inspiration for the schools to define their needs and preferences to focus on specific aspects.

Table 2. Behavioural goals regarding physical activity and healthy nutrition.

\begin{tabular}{|c|c|}
\hline Physical activity & Healthy nutrition \\
\hline $\begin{array}{l}\text { - Children engage in moderate to vigorous physical activity (MVPA) } \\
\text { for at least } 60 \mathrm{~min} / \text { day. }\end{array}$ & $\begin{array}{l}\text { - Children consume a healthy } \\
\text { breakfast every day. }\end{array}$ \\
\hline $\begin{array}{l}\text { - Children use active school transport (cycling, walking) if the } \\
\text { distance to their school is less than } 2 \mathrm{~km} \text {, or children are taken to } \\
\text { school by active transport. }\end{array}$ & $\begin{array}{l}\text { - Children consume a healthy lunch } \\
\text { every day. } \\
\text { - Children consume two (different) }\end{array}$ \\
\hline $\begin{array}{l}\text { - Children are physically active (MVPA) for at least } 20 \text { min/day during } \\
\text { school breaks. }\end{array}$ & $\begin{array}{l}\text { pieces of truit a day. } \\
\text { - Children consume 100-200 grams of } \\
\text { vegetables a day. }\end{array}$ \\
\hline $\begin{array}{l}\text { - Children are not sedentary for more than } 30 \text { consecutive minutes. } \\
\text { Every } 30 \text { minutes, children should have a 2-minute break involving } \\
\text { walking, standing or moving. }\end{array}$ & $\begin{array}{l}\text { - Children replace energy-dense } \\
\text { snacks with healthier alternatives. } \\
\text { - Children drink water instead of }\end{array}$ \\
\hline $\begin{array}{l}\text { - Children are physically active (MVPA) for at least } 20 \mathrm{~min} / \text { day during } \\
\text { physical education lessons (lasting } 1 \text { hour) at least three times a } \\
\text { week. }\end{array}$ & $\begin{array}{l}\text { sugar-sweetened drinks. } \\
\text { - Children do not drink sports or } \\
\text { energy drinks. }\end{array}$ \\
\hline \multicolumn{2}{|l|}{$\begin{array}{l}\text { - Children do not have more than } 2 \text { hours/day of sedentary screen } \\
\text { time (television/computer/tablet). }\end{array}$} \\
\hline \multicolumn{2}{|l|}{$\begin{array}{l}\text { - Children take part in afterschool physical activities (e.g. sports } \\
\text { clubs, afterschool physical activity programmes, and free time } \\
\text { outdoor play). }\end{array}$} \\
\hline $\begin{array}{l}\text { - Children are physically active (MVPA) for at least } 60 \mathrm{~min} / \text { day during } \\
\text { the weekend. }\end{array}$ & \\
\hline
\end{tabular}

\section{Interviews}

Semi-structured interviews are conducted with the school coordinator and the health promoter in each school. The behavioural goals are used as topics in these interviews. The aim of the interviews is to draw up an overview of the HP elements in the school and a broad understanding of any dominating organizational issues in the school. The results are summarized in an overview, checked by the interviewees, and fed back to the project team.

\section{Barrier questionnaire}

To examine which factors in the context are perceived to be potential barriers for the process of change, all teachers and external pedagogical staff are asked to complete a questionnaire. The used questionnaire was based on the Measurement Instrument for Determinants of Innovations (MIDI), a Dutch validated questionnaire developed by Fleuren et al. [71] and used in several Dutch studies [77, 78]. The questionnaire contains 46 statements, related to innovation-, implementers-, organization-, and socio-political 
context-related barriers for HPSF affecting innovation adoption, implementation, and integration. Responses to each statement range from 1 (totally disagree) to 10 (totally agree). Statements with an average score below 6 are defined as potential barriers. This corresponds to the grading system used in Dutch primary schools, which also uses a range from 1 to 10 for school tests, and scores below 6 as insufficient or fail.

\section{Practices questionnaire}

A questionnaire, based on previous work by Gevers et al. [79] and O'Connor et al. [80], is used to examine HP practices of teachers at school and parents at home, e.g., rules, modelling behaviour, encouragement, and availability. The existing questionnaires were used in several previous Dutch studies [81, 82]. The items in the questionnaire focus on parents in the home setting. Therefore, we rephrased the items in the teacher questionnaire to the school setting. To deal with validity and reliability concerns, we pre-tested the instruments, and we will check for variability per question and will calculate Cronbach's Alpha afterwards. A Likert-scale from 1 (completely disagree) to 5 (completely agree) is used for the answers.

\section{Health and behavioural measures}

Measurements of children's health and health behaviours are carried out by researchers during one week at the beginning of the school year [55]. Inter-rater variability was minimised by training the researchers according to a strict protocol. The children wear an accelerometer for the whole week to objectively assess their PA levels. Their height, weight, and waist and hip circumference are measured during the physical education lessons. Children's dietary and PA behaviours are assessed using a questionnaire for the children during class hours and a digital questionnaire for their parents. Ethical approval (14-N-142) was given by the Medical Ethics Committee Zuyderland located in Heerlen (Parkstad, the Netherlands). Parents had to sign an informed consent to participate in all measurements for themselves and their child(ren). More detailed information about these measurements has been published by Willeboordse et al. [55].

How does the process of change in each school evolve and which factors affect this process?

The second key question aims to examine the process of change, i.e., the adoption, implementation, and integration of the HP changes, and its interaction with the school context. Since HPSF takes place in a complex system, some aspects are important to take into consideration. First, the process of change in each school may not be characterized by a linear cause-effect relationship. This non-linearity means that small changes can produce large effects at a so-called 'tipping' point $[48,70]$. When this 
tipping point is reached is hard to predict. Therefore, both the innovators in the school and all external experts involved need to be receptive to what emerges and expect the unexpected. Second, a complex system means adaptation: interacting elements and people in an environment respond and adapt to each other [48]. This means that what emerges in a school is interpreted as a function of on-going adaptations that may continually lead to new needs, interests, and opportunities in the school. In this context, Reiser et al. [73] stated that the implementation of a change is more successful and leads to greater ownership and commitment if it involves a process of mutual adaptation. This indicates a bidirectional process in which a proposed change is modified to suit the needs, interests, and opportunities of the school, and in which the people at the school are open to (major) adjustments and adjust to meet the requirements of that change. In essence, this requires a combined top-down/bottomup process, which is another aspect to take into consideration when changing a complex system. A bottom-up approach is needed as teachers, children, and their parents know best which changes are most appropriate to their school. Hence, they should be the ones to lead the process of change. A top-down approach is needed as the external experts involved have specific health promotion knowledge, skills, and experiences, which may lead to more effective changes $[70,74]$. It is important not only to find a balance between these two, but also to be constantly aware of the primary source of the idea for a change as well. When an idea develops bottom-up, the external experts should help the school by using their specific knowledge and experiences. When the primary idea is introduced top-down, the external experts should help the school to encourage involvement among the people in the school and help them make contextual adjustments to fit the proposed change to the system.

To facilitate this on-going process of change in each school, we evaluate the process by continuously monitoring the changes and their consequences. The results have to be concrete and specific, so useful feedback and recommendations can be given to the schools to guide their actions and help them adapt to the changes. In the course of the study, all monitoring results are combined to create more abstract and general recommendations to help other people or organizations who want to start HP initiatives in schools. The process of change is monitored using mixed methods.

\section{Interviews}

Annual interviews are conducted with the school coordinator and the health promoter of each school to discuss the HP changes in the school, their development and implementation, and the influencing factors associated with them.

\section{Observations}

A researcher participates, observes, and takes notes in all meetings of the project team and meetings of the health promoters. This researcher also conducts observations in the four schools, with the aim of learning about the school's dynamics and to see and 
hear influencing factors of HPSF (rather than as a form of fidelity assessment). To create openness, no observational checklist is used. During these school visits, the researcher randomly talks to staff and children in the school to hear about their experiences and perceptions regarding HPSF. Observations will take at least one full week each year during effect measurements and frequent visits (at least once every three months) to each school during the year. Notes will be taken during and immediately after visiting the school. All observational notes provide qualitative data about HPSF and any experienced influencing factors.

\section{Barrier questionnaire}

It is expected that different barriers will appear during different phases in the process of change. Therefore, twice a year, all teachers and external pedagogical staff are asked to complete the same questionnaire as described above to address the first key question. Statements with an average score below 6 are defined as possible barriers. Some open questions have been added to obtain the respondents' opinions on how the process is going.

\section{Practices questionnaire}

The process of change can also have an impact on the practices of teachers and parents. Therefore, the same questionnaire as described above to address the first key question is annually filled in by the teachers and parents.

\section{How can research contribute to the process of change?}

The third key question is intended to examine supportive contributions of research to the process of change at the schools. This is achieved by embedding the research in the HPSF initiative, similar to its role in action research [75]. As a result, evaluation is no longer merely an external observation of strategies to implement changes, but becomes one of the strategies itself [48]. The attitude of the researchers in this approach is also different: they are no longer neutral and fully objective, but join in the discussions and give support to the innovators whenever possible on the basis of their professional knowledge, skills, and experiences, as well as the results of the monitoring data [48]. It is expected that regular feedback will provide valuable guidance to the process of change in the schools [75]. Examples of feedback from the researchers to each school are written summaries of the most important results of the interviews; overviews of the perceived barriers for the teachers and external pedagogical staff; and short, easily understandable animated videos of the most important results of the health and behavioural measures. Furthermore, as part of the feedback, we aim to include suggestions for focal points to further improve HPSF. Other contributions of the researchers on top of the feedback they provide are the suggestions for possible 
behavioural goals and the offer of a selection of relevant, previously developed, and evidence-based additional changes for the schools. To be able to offer this as a range of possibilities, a so-called 'fruit basket model' is introduced. This 'basket' consists of a continuously expanding overview of available evidence-based additional changes ('fruits') that schools can introduce. Examples of such changes are gardening activities, energizers in the lessons, and creating a PA-friendly schoolyard. According to the school's needs, interests, and opportunities, the school coordinator decides together with the working groups what the school's focus will be, and which specific 'piece of fruit' - additional change-fits their school. This change is then adapted to the specific school context, with the help of external experts, before implementation starts. An overview of the current 'fruit basket' is presented as Table S1 (Additional file 1). Questions regarding the researchers' contributions are included in the interviews to evaluate the extent to which the contributions are experienced as supportive, and/or whether other contributions are desirable.

Do children's health and health behaviours improve as a result of the HP changes?

The fourth key question is intended to determine the influence of HPSF on children's health and health behaviours to examine how, for whom, and in what circumstances the initiative works. To examine if HPSF leads to changes in children's health and health behaviours, an effect study is being carried out with a quasi-experimental design [55]. Data on children's health and health behaviours are gathered during annual measurement weeks between 2015 and 2019 at all four HPSF schools and at four control schools (approximately 1700 children, 900 parents, and almost 80 employees). However, changes may have different effects in different contexts, even if their implementation does not vary [47]. Therefore, to investigate for whom and under which circumstances the changes have the greatest effect, we examine the differences in effect between the schools by combining the results of the effect evaluation with relevant implementation and contextual factors of the schools. The implementation and contextual variables which are collected quantitatively at both the intervention and control schools, e.g., characteristics of the student population and HP practices of teachers and parents, are included as potential moderators in the analyses. Moderator analyses have been defined as a fundamental step in understanding behaviour change and are conducted by using an interaction term in the statistical models and (in the case of significant interactions), stratifying the data by a moderator to re-examine the effect [83]. The quantitative implementation and contextual data that are only collected in the four intervention schools, e.g., perceived barriers as measured by the questionnaire, are analysed on changes over time and compared between the schools. Finally, qualitative implementation and contextual data that are collected in the four intervention schools, 
e.g., school specific influencing factors on the process of change, are used in a comparative manner between the schools to provide additional insight or so-called illumination on the process of change [84].

\section{Discussion}

The present paper has introduced the contextual action-oriented research approach that we developed to deal with the complexity of both the school system and the HPSF initiative. The paper has shown how we aim to contribute to the initiative and at the same time conduct a thorough evaluation. Some methodological, practical, and/or integrity limitations and strengths of the approach need to be discussed.

First, fully assessing and understanding all aspects of each context, the process of change, and the implementation of each change, is impossible due to limitations of time, resources, and participant burden [47]. Therefore, CARA researchers have to make difficult selection choices about which data to collect and in how much detail. Luckily, we do not have to start from scratch. We build on previous work published in the international literature on, e.g., relevant concepts in the process of change in complex (school) systems, which was essential for this decision process. We included methods that are appropriate to obtain rich information, that are feasible for the researchers and the schools, and that can be translated into rapid and real-time feedback for the schools.

Second, we believe that the data collection instruments in CARA need to fit the context to be able to get meaningful data. Therefore, existing instruments might not be available or have to be adapted to the context. In the current study, we have adopted the strategy of preferring adjustment of existing and tested instruments over the development of new instruments. By using the principle of data triangulation in our analysis, we combine the accuracy of the quantitative questionnaires with the in-depth insights that interviews and observations afford.

Third, CARA includes not only an evaluation of the process of change in the schools, but also an effect study to investigate the evidence for the behavioural and health effects of these changes among the children. Since randomization is neither desirable nor feasible here [49], a quasi-experimental study design is used. To investigate for whom and under which circumstances the changes cause the greatest effect, the school-specific effects are combined with relevant process and contextual factors at each school. Although we do recognize the importance of assessing the implementation fidelity, the focus in our study is not on the fidelity of intervention components, but on identifying when and how adaptation take place, and which factors prove to be crucial for sustained changes. This specific focus of interest is based on the notion that even small changes may produce large effects in a specific context (i.e., 'tipping' point) [48, 70]. Better (i.e., high fidelity) implementation of a change does thereby not necessarily 
mean greater effect [47]. Another aspect to consider is that CARA researchers are not external observers, but actively participating partners in the initiative. By including an effect study with a quasi-experimental design in CARA as well, we believe we combine the best of two worlds: the advantages of a researcher involved in the process of change and still being able to study the effects objectively.

Finally, CARA also implies time-consuming research, as a thorough insight into the school context is necessary. This insight requires a relationship of trust between the researchers, the schools, and all other partners involved, which takes time to build. In this relationship, nobody should be afraid to say what really bothers them, which should yield data that reflect the real situation. Moreover, CARA requires flexible time planning of the researchers; they need to be able to react quickly to what happens in each school, to be able to give relevant support, and to analyse the data quickly to be able to give rapid and real-time feedback. At the same time, the feedback process needs to take place in a careful manner, as both the initiative and the research can benefit from an open discussion of the real situation of those involved without losing the trust of the informants. To maintain a relationship of trust, we aim to offer honest but discrete feedback. When results cannot be fed back anonymously, we aim to ask permission of the informants before communicating the feedback to others. CARA can also be timeconsuming for the schools. Researchers need to consider this and ensure that studying the initiative is feasible for them and the schools. Due to our critical selection of mixed methods, we believe we have found a feasible way to support and evaluate the initiative in the schools. By means of the feedback provided and the focal points included to further improve HPSF, we aim to offer added value to the schools which we hope outweighs their time investment.

In addition to these methodological, practical, and integrity aspects, there is another important aspect to consider: whereas the current paper mainly focuses on the school complexity, the school is only one of a child's microsystems. Changes in children's home setting and neighbourhood, their other microsystems, also interact with the impact of changes at school [16]. Thus, the complexity goes beyond the focus of the research described in this paper.

\section{Conclusion}

Overall, we think that CARA is a possible solution to the challenge of supporting and evaluating change in school-based initiatives. CARA generates knowledge and experiences on how to deal with health promotion in complex systems. This paper shows an innovative approach to contribute to the process of a complex intervention, including a thorough evaluation of the process and its final outcomes that addresses the importance of the implementation context. We think that CARA can be an example for research efforts in comparable initiatives and can help to make sustainable (add-in) changes in complex systems. 


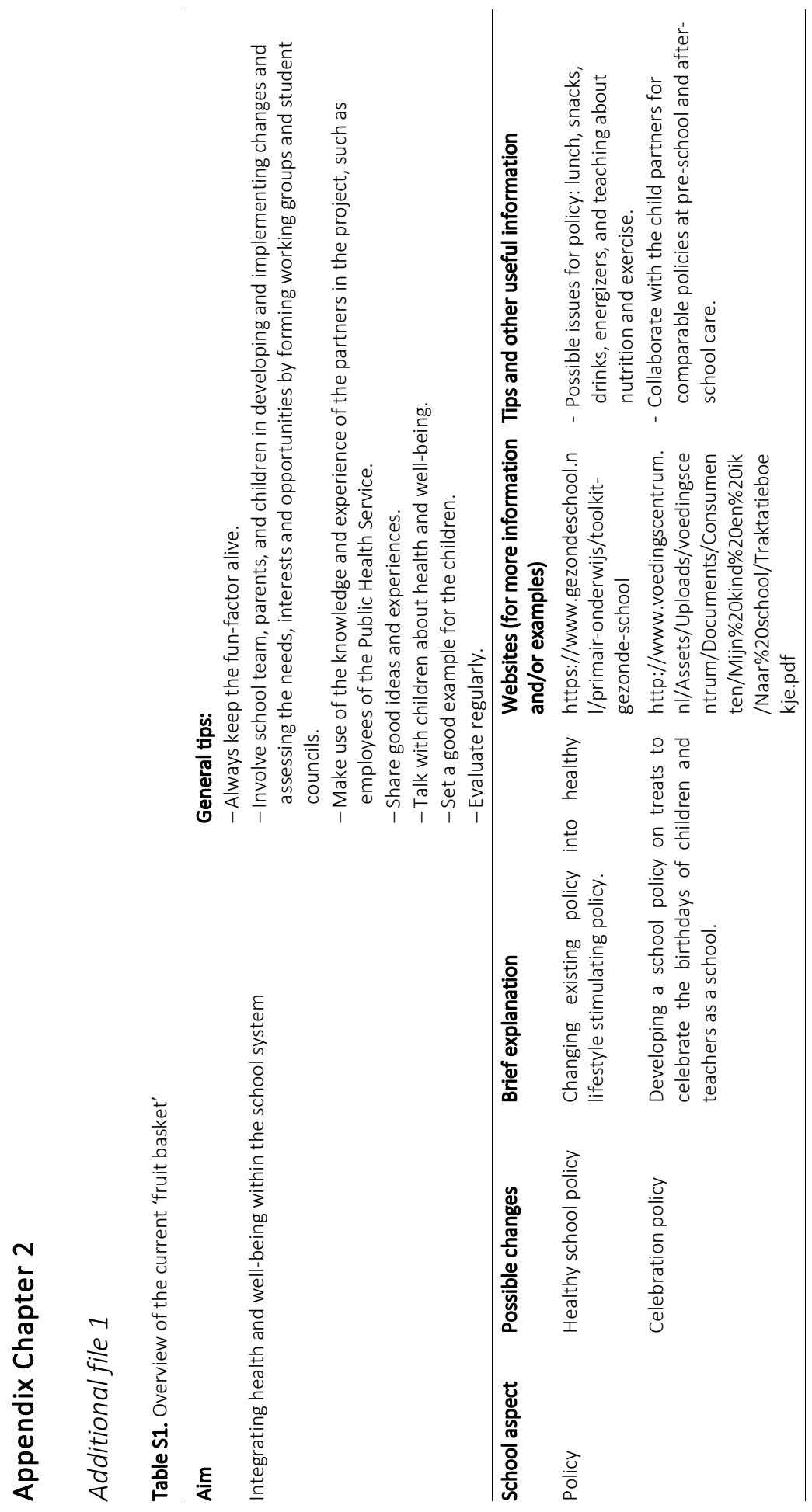




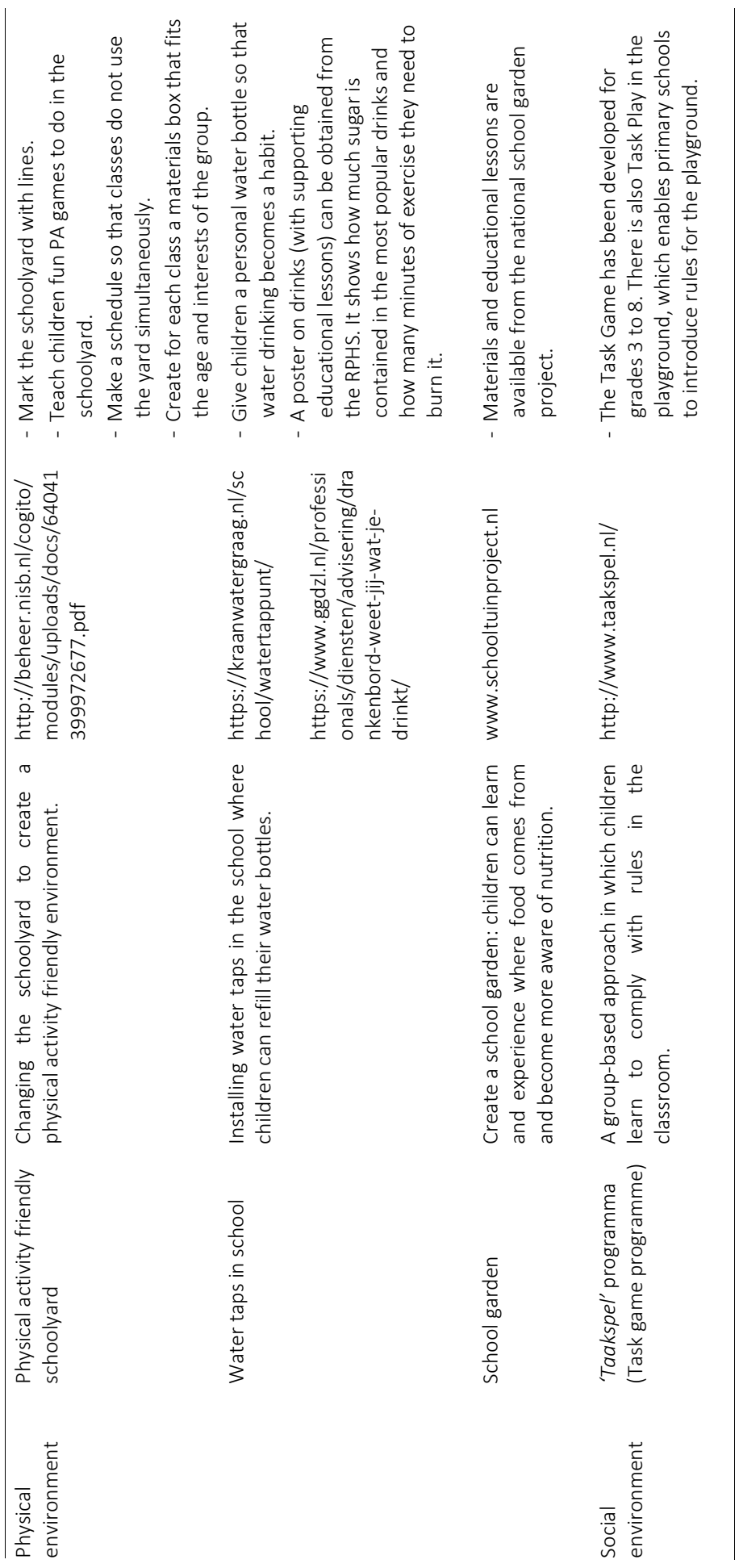




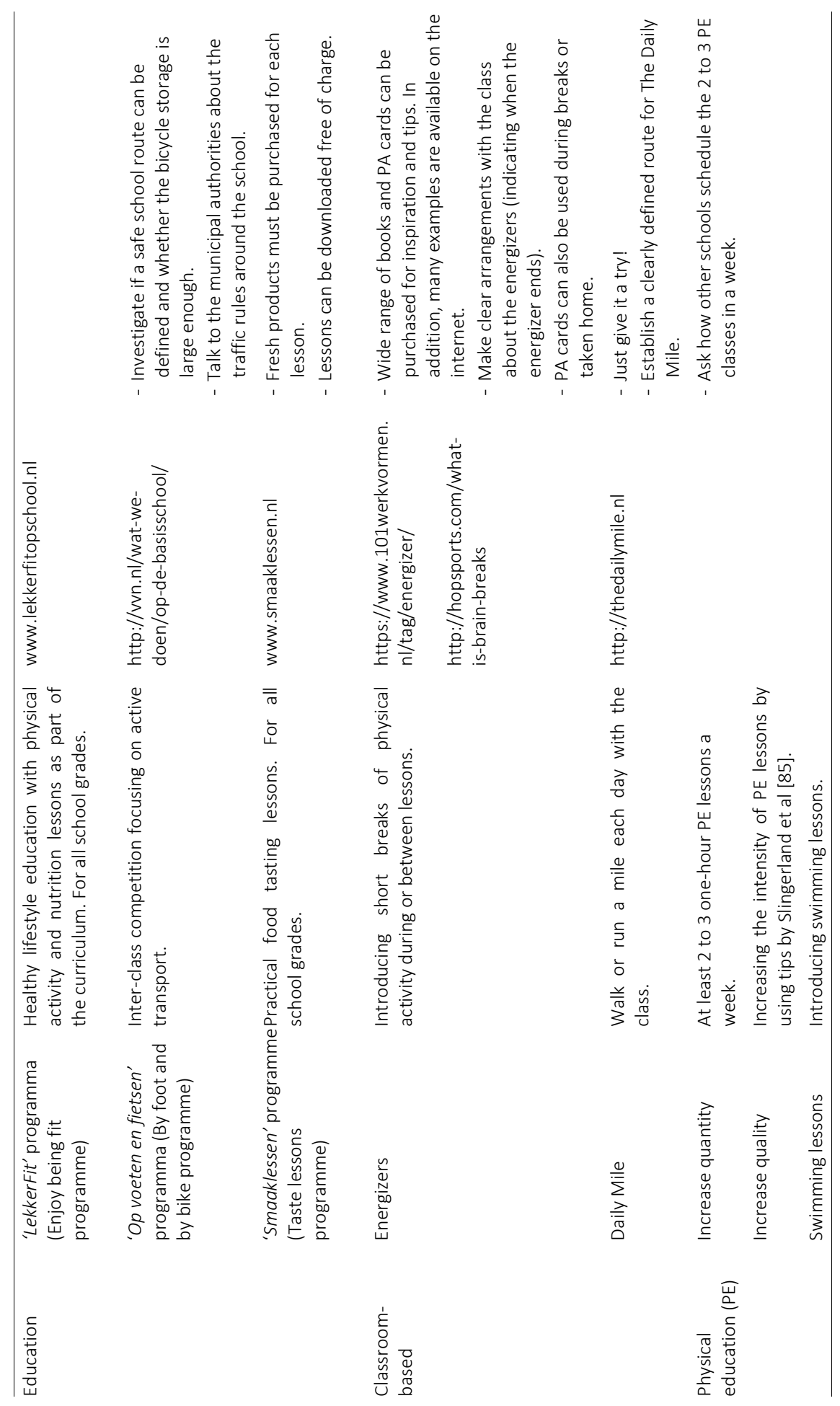




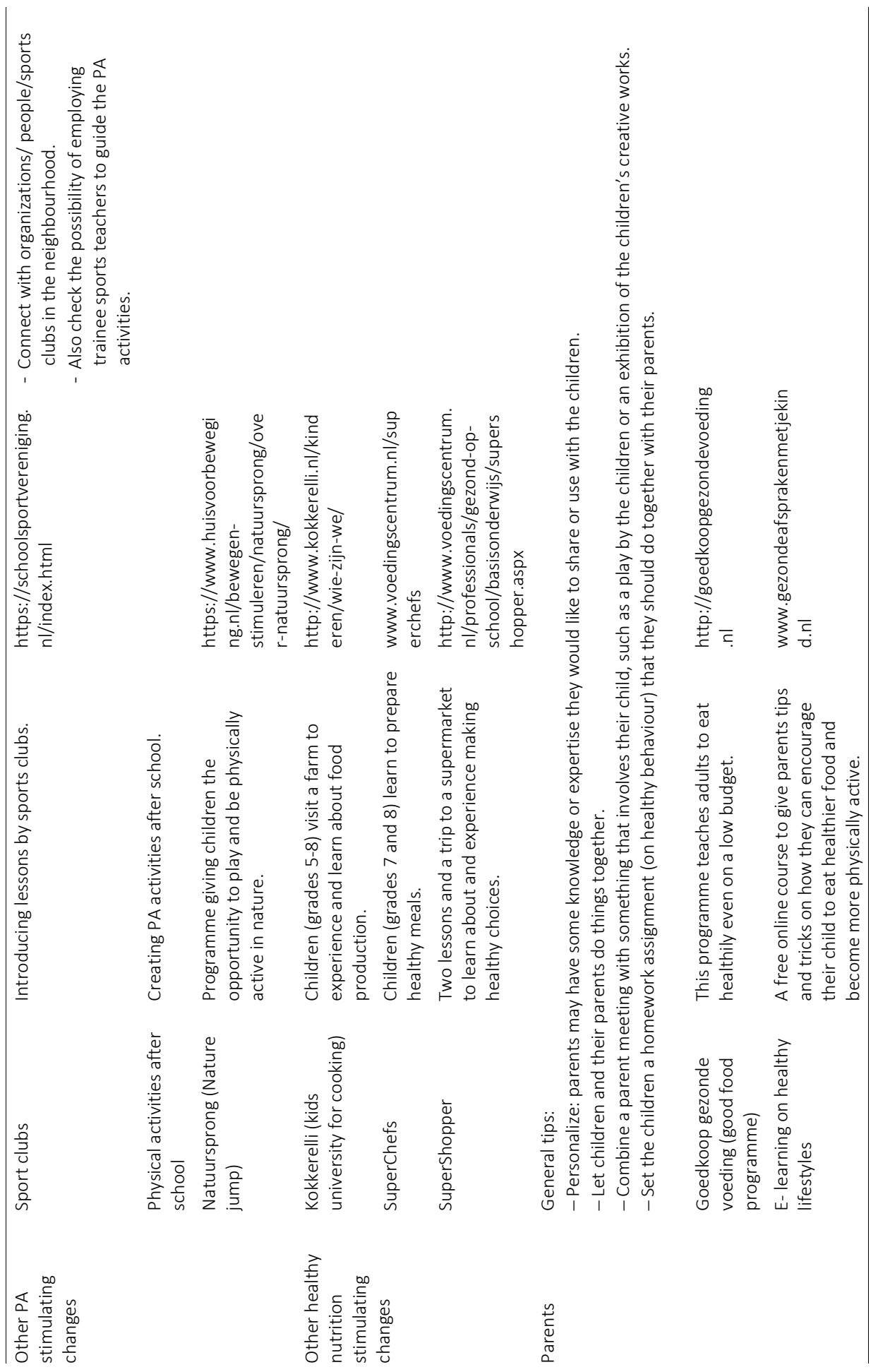


A contextual action-oriented research approach

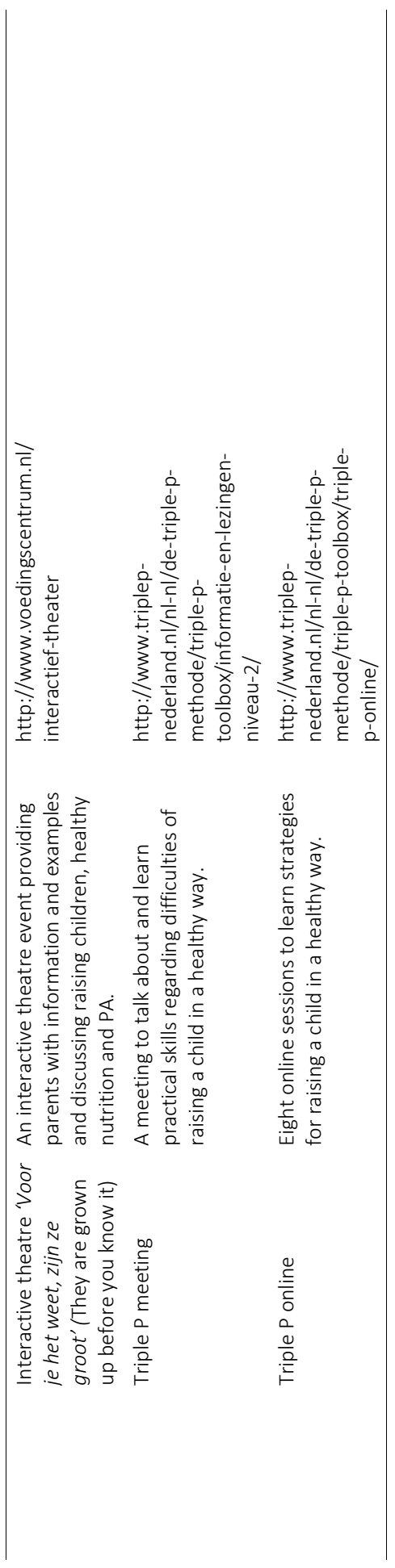





\section{Chapter 3}

\section{Process evaluation of the Healthy Primary School}

of the Future: The key learning points

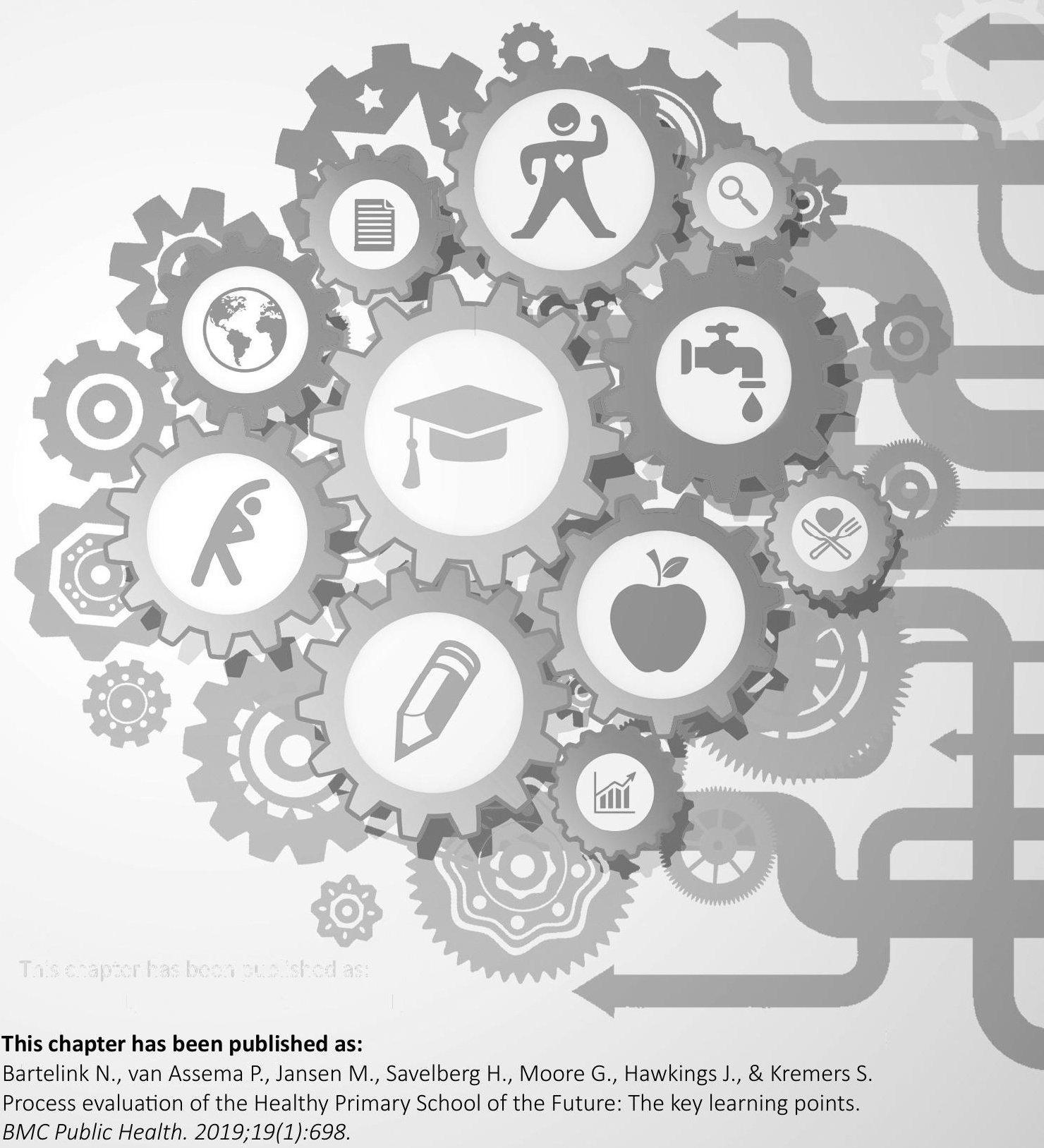




\section{Abstract}

\section{Background}

While schools have potential to contribute to children's health and healthy behaviour, embedding health promotion within complex school systems is challenging. The 'Healthy Primary School of the Future' (HPSF) is an initiative that aims to integrate health and well-being into school systems. Central to HPSF are two top-down changes that are hypothesized as being positively disruptive to the Dutch school system: daily free healthy lunches and structured physical activity sessions. These changes are expected to create momentum for bottom-up processes leading to additional healthpromoting changes. Using a programme theory, this paper explores the processes through which HPSF and the school context adapt to one another. The aim is to generate and share knowledge and experiences on how to implement changes in the complex school system to integrate school health promotion.

\section{Methods}

The current study involved a mixed methods process evaluation with a contextual action-oriented research approach. The processes of change were investigated in four Dutch primary schools during the development year (2014-2015) and the first two years of implementation (2015-2017) of HPSF. The schools (each with 15-26 teachers and 233-389 children) were in low socioeconomic status areas. Measurements included interviews, questionnaires, observations, and analysis of minutes of meetings.

\section{Results}

Top-down advice, combined with bottom-up involvement and external practical support were key facilitators in embedding HPSF within the schools' contexts. Sufficient coordination and communication at the school level, team cohesion, and feedback loops enhanced implementation of the changes. Implementation of the healthy lunch appeared to be disruptive and create momentum for additional health-promoting changes.

\section{Conclusions}

Initiating highly visible positive disruptions to improve school health can act as a catalyst for wider school health promotion efforts. Conditions to create a positive disruption are enough time, and sufficient bottom-up involvement, external support, team cohesion and coordination. The focus should be on each specific school, as each school has their own starting point and process of change. 


\section{Background}

The school setting has the potential to influence children's health and well-being, in part by supporting the adoption of healthy behaviours [14, 15, 86]. Establishing healthy behaviours at an early age may help to improve children's health and educational achievements; both may lead to improved health later in life and closing the equity gaps in both health and academic achievement $[58,87]$. However, school health promotion is globally often characterised by relatively low priority, fragmentation, and a lack of coordination $[19,25]$. The Health Promoting School framework as defined by the World Health Organization aims for a whole-school approach, and focuses on reorienting school systems toward health promotion through embedding health and well-being in the curriculum, creating healthy social and physical environments and engaging with parents and the wider community [21]. This concept has shown promise, though several studies (including the Netherlands) indicate that effects are often hampered by underestimation of the challenges associated with implementing meaningful wholesystem changes [20,32, 42, 88].

Challenges associated with changing school systems vary between schools: every school has its own dynamics, shaped by a large number of interacting elements and ever-changing agents within it [32, 42,47]. Schools can thus be conceptualised as complex systems. Key to this conceptualization is an understanding of the non-linearity of systems and the ways in which feedback impacts overall system behaviours and adaptations over time [89]. An intervention can be seen as an attempt to positively disrupt the prior functioning of a system [54, 90]. Moreover, complexity goes beyond the school gates, as school is only one of a diversity of microsystems which interact to shape child development and wellbeing. Changes in children's home setting and neighbourhood, other microsystems with which children interact, also interact with the impact of changes at school [16].

A Dutch initiative based on the Health Promoting School framework, and informed by a systems approach, is the 'Healthy Primary School of the Future' (HPSF). This initiative, with a focus on healthy nutrition and physical activity (PA), aims to improve children's health and well-being by enhancing health promotion throughout the whole school system, with the aim of contributing to fostering a healthier future generation $[55,91]$. Central to this HPSF-concept is the top-down initiation of two changes, a free healthy lunch each day and daily structured PA sessions. While in other national school systems these may represent usual practice, the two changes are hypothesized as being positively disruptive to the Dutch school system. In the Netherlands, children eat their lunch at home or bring lunch to eat at school; PA is restricted to one or two physical education classes a week and some free playtime during (lunch) breaks. Contextualization of the two changes is supported by bottom-up involvement of teachers and parents. The changes aim to facilitate the conditions within the school 
context for healthy dietary and PA behaviours and to create momentum for more bottom-up processes that lead to additional health-promoting (HP) changes.

To better understand implementation processes [88], we conducted a process evaluation. In line with recent debates in this research area $[47-49,52]$, the focus in this process evaluation was not on the fidelity of intervention components in purely compositional terms, but on adaptation of the intervention and system to one another, and factors crucial for sustained change $[88,92]$. The aim of the current study was to generate knowledge and experiences on how to implement changes in the complex school system to integrate school health promotion and to share key learning points. Specifically, the study explored the processes through which HPSF and the school context adapted to one another during the development year (academic year 2014/15) and the first two years of implementation (2015/17) in four schools. Three main research questions were formulated: 1) What was the pre-existing context of the four schools prior to the introduction of HPSF?, 2) How was HPSF developed and implemented and how did it interact with the context of the four schools?, and 3) After two years, to what extent was HPSF integrated and did the context of the four schools change?

\section{Methods}

\section{Study design}

This process evaluation is part of an overall study that investigates HPSF using a quasiexperimental study design [55]. The overall study includes four intervention schools and four control schools. The process evaluation reported here focuses on the four intervention schools, using mixed methods (Table 1). Data were collected during three years (2014-2017) in four intervention schools. Ethical approval was obtained from the Medical Ethics Committee Zuyderland located in Heerlen (the Netherlands). All four schools started implementation of HPSF in November 2015. Funding for implementation is provided until the end of 2019. However, the four schools have committed to continued implementation after 2019 and to making the changes sustainable in their school. 
Table 1. Research questions (RQ) and used methods

\begin{tabular}{|c|c|c|c|}
\hline \multicolumn{2}{|l|}{$\mathrm{RQ}$ and sub-RQ } & Concepts and variables & Methods \\
\hline \multicolumn{2}{|c|}{$\begin{array}{l}\text { RQ1: What was the pre-existing context of the four } \\
\text { schools prior to the introduction of HPSF? }\end{array}$} & $\begin{array}{l}\text { The school context: } \\
\text { - HP practices of } \\
\text { teachers/parents } \\
\text { - } \text { HP elements in school } \\
\text { - Dominant organizational } \\
\text { issues } \\
- \text { Perceived barriers for } \\
\text { HPSF } \\
\text { - } \text { Characteristics of school } \\
\text { population }\end{array}$ & $\begin{array}{l}\text { - Interviews } \\
\text { - Minutes } \\
\text { - Observations } \\
\text { - Practices_Q } \\
\text { - Barrier_Q } \\
\text { - Open questions } \\
\text { in Barrier_Q }\end{array}$ \\
\hline \multirow{4}{*}{$\begin{array}{l}\text { RQ2: How was } \\
\text { HPSF developed } \\
\text { and } \\
\text { implemented } \\
\text { and how did it } \\
\text { interact with the } \\
\text { context of the } \\
\text { four schools? }\end{array}$} & $\begin{array}{l}\text { 2.1: How were the two top- } \\
\text { down changes developed and } \\
\text { implemented in the four } \\
\text { schools? }\end{array}$ & $\begin{array}{l}\text { Two top-down changes: } \\
\text { - Daily healthy lunch } \\
\text { - Structured PA sessions }\end{array}$ & $\begin{array}{l}\text { - Interviews } \\
\text { - Minutes } \\
\text { - Observations }\end{array}$ \\
\hline & $\begin{array}{l}\text { 2.2: To which additional health } \\
\text { promoting changes did the two } \\
\text { top-down changes lead to in the } \\
\text { four schools? }\end{array}$ & $\begin{array}{l}\text { Changes in HP elements in } \\
\text { school: } \\
\text { - School routine } \\
\text { - Policy } \\
\text { - Education } \\
\text { - Environment }\end{array}$ & $\begin{array}{l}\text { - Interviews } \\
\text { - Minutes } \\
\text { - Observations }\end{array}$ \\
\hline & $\begin{array}{l}\text { 2.3: Which (potential) barriers } \\
\text { for HPSF were perceived by the } \\
\text { implementers in the four } \\
\text { schools, and how did they } \\
\text { change during the first two } \\
\text { years of implementation? }\end{array}$ & $\begin{array}{l}\text { Perceived (potential) } \\
\text { barriers for HPSF: } \\
\text { - Innovation-related } \\
\text { - Implementers-related } \\
\text { - Organization-related } \\
\text { - Socio-political context- } \\
\text { related }\end{array}$ & - Barrier_Q \\
\hline & $\begin{array}{l}\text { 2.4: Which factors influenced } \\
\text { the development and } \\
\text { implementation of HPSF in the } \\
\text { four schools during the first two } \\
\text { years of implementation? }\end{array}$ & $\begin{array}{l}\text { Development and } \\
\text { implementation process of } \\
\text { HPSF: } \\
\text { - Coordination } \\
- \text { Team cohesion } \\
- \text { Bottom-up involvement } \\
- \text { External support } \\
- \text { Momentum }\end{array}$ & $\begin{array}{l}\text { - Interviews } \\
\text { - Minutes } \\
\text { - Observations } \\
\text { - Open questions } \\
\text { in Barrier_Q }\end{array}$ \\
\hline \multirow[t]{2}{*}{$\begin{array}{l}\text { RQ3: After two } \\
\text { years, to what } \\
\text { extent was HPSF } \\
\text { integrated and } \\
\text { did the context } \\
\text { of the four } \\
\text { schools change? }\end{array}$} & $\begin{array}{l}\text { 3.1: What impacts did HPSF give } \\
\text { rise to in the four schools after } \\
\text { the first two years of } \\
\text { implementation? }\end{array}$ & $\begin{array}{l}\text { Changes in the school } \\
\text { context: } \\
\text { - HP elements in school } \\
\text { - HP practices of } \\
\text { teachers/parents } \\
- \text { Characteristics of school } \\
\text { population }\end{array}$ & $\begin{array}{l}\text { - Interviews } \\
\text { - Minutes } \\
\text { - Observations } \\
\text { - Practices_Q }\end{array}$ \\
\hline & $\begin{array}{l}\text { 3.2: To what extent was HPSF } \\
\text { seen as being fully integrated } \\
\text { into the everyday functioning of } \\
\text { the school after the first two } \\
\text { years of implementation? }\end{array}$ & $\begin{array}{l}\text { Perceived feelings of } \\
\text { integration }\end{array}$ & - Interviews \\
\hline
\end{tabular}




\section{Programme theory}

This study uses a contextual action-oriented research approach (CARA) [92]. CARA focuses on contextual differences, use of monitoring and inducing feedback loops to support and evaluate the processes of change in each school. Based on the principles of CARA and complex systems thinking, we developed a programme theory on the hypothesized processes of how HPSF integrates into the school context (Figure 1). The HPSF-concept and the pre-arranged financial and practical support for its implementation, aim to act as an 'event' that positively disrupts the pre-existing dynamics in the school context $[47,54]$. The context within and across schools acts as the starting point of HPSF [48]. Therefore, understanding relevant aspects of the preexisting school context is required, such as HP practices of teachers and parents [42], HP elements in school (school routine, policy, education, and environment) [21], dominating organizational issues (e.g., staff turnover) [68], barriers for HPSF related to innovation, implementers, organization, and socio-political context [71], and characteristics of the school population (demographics, health behaviours, health and well-being) [68]. The introduction of HPSF into the school context initiates the HPSF process of development, implementation, and integration [93]. Based on existing implementation literature it was hypothesized that coordination, team cohesion, bottom-up involvement, and external support would improve this process [20, 94-96]. During the process, feedback loops will develop in two directions [89]: on the one hand, the school context is expected to impact the HP change process, on the other hand, the context may respond to HP changes, which may result in a new way of working in the school context $[42,71]$. Feedback loops may be positive, thereby amplifying the changes, or negative, thereby counteracting the changes $[42,89]$. During this complex process of change, the system tries to find a new balance: it tends to self-organise to a new state of stability, either by pushing the change out of the system or by integrating the change into the system [97]. A key assumption of our programme theory concerns non-linearity in the cause-effect relationship, which means that small changes can produce large effects at a so-called 'tipping' point [48, 70]. Furthermore, the loop in the bottom of Figure 1 visualises the hypothesis that realized changes may shift system norms toward a focus on health and well-being, thereby creating momentum for additional HP changes [97]. Finally, a moderating effect of the context on child outcomes is visualised in the right-top of the figure: even when schools implement similar changes, the impact may differ by school [47]. 


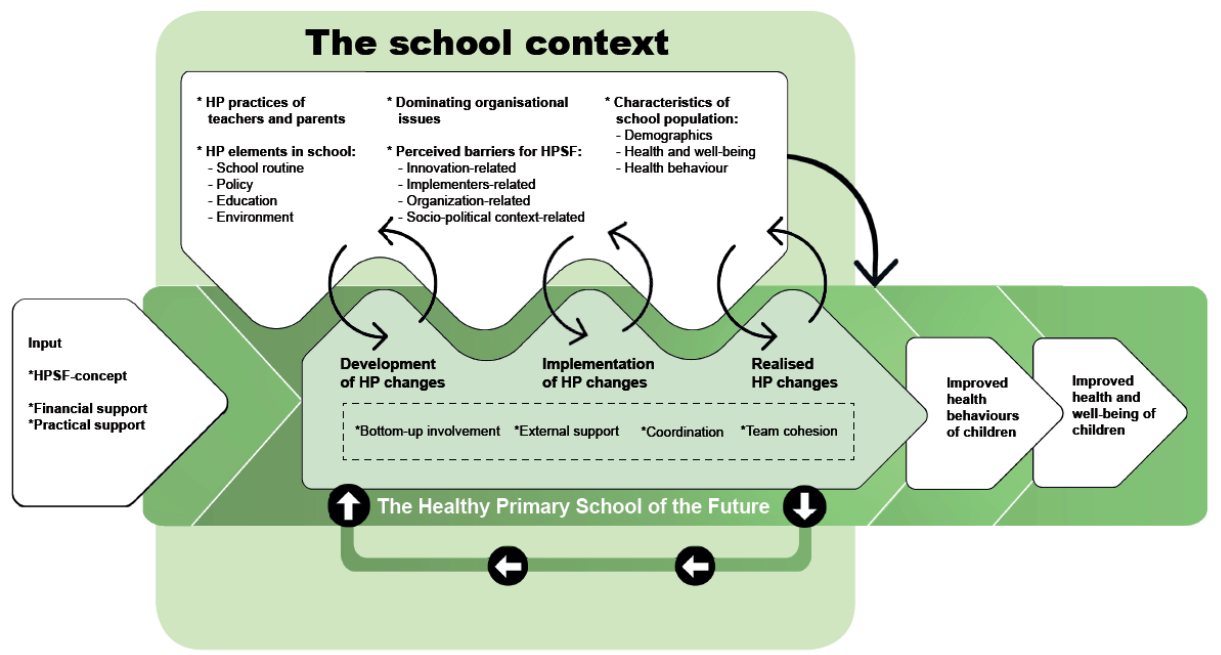

Figure 1. Programme theory

\section{Participating schools (S1-S4)}

The four schools were members of the regional educational board 'Movare' situated in the Parkstad region in the southern part of the Netherlands. This region has a low average socioeconomic status (SES), and unhealthy behaviours and overweight are highly prevalent compared to the rest of the Netherlands [40,62]. More information on the recruitment of the schools and participants is described in Willeboordse et al. [55].

\section{The Healthy Primary School of the Future}

The worrying increase in unhealthy behaviours among their schoolchildren and the fragmentation of school health promotion, induced Movare to initiate collaboration with the regional Public Health Services and Maastricht University. Together they developed the idea for the HPSF initiative [55]. The provincial authorities supported the initiative financially. The two changes (providing a lunch each day and structured daily PA session) were implemented by external pedagogical employees (PE) provided by childcare organizations, to avoid increasing the workload of teachers. This integration of the childcare organization during school hours, is intended to change the school's organization in a sustainable way. The aim for the future is to bring school and childcare closer together and thereby create an integrated day for children, whereby children are supervised by the same people prior, during and after school hours. The above mentioned commitment of schools and childcare organizations to continued implementation, also includes this employment of external pedagogical employees during school hours. 
The lunch products were provided by catering services and instructions for PA sessions were provided by a sports and leisure organization. The schools involved teachers and parents in the adoption decision and the process of adapting the two changes to the school context. The schools decided to start implementation of HPSF only if they had full teacher support, which was orally assessed during team meetings, and at least $80 \%$ parental support, which was assessed by a paper-based survey, asking whether they support the change, and if not, why. Each school selected a teacher as school coordinator, who managed HPSF in their school. A PE coordinator per school acted as contact person for all external PE in that school. A health promoter from the regional Public Health Services was assigned to each school to provide support when needed. In this pilot, researchers from Maastricht University monitored and fed back results to the schools to support the processes of change. Each school initiated regular meetings to discuss their processes of change, such as meetings between the school coordinator and PE coordinator, and working groups with teachers and parents, as well as children's voice groups. The health promoters of the four schools also met regularly to keep each other updated on the on-going processes of each school. Overarching the four schools, the HPSF initiative was led by a project leader from Movare and an executive board with representatives of the three collaborating organizations, including the project leader. A project team was created with representatives of all partners involved: the four schools, Movare, regional Public Health Services, Maastricht University, the Limburg provincial authorities, childcare organizations, the caterer, and the sports and leisure organization. More details about the HPSF initiative were published elsewhere [55].

\section{Mixed methods}

\section{Interviews}

Qualitative in-depth data were collected using semi-structured interviews. At the end of the first two academic years (2014/15 and 2015/16), interviews were held in each school with the school coordinator and the school health promoter together. The interviews aimed to get an overview of the school's current HP elements (school routine, policy, education, environment), and an understanding of any dominating organizational issues. Notes were taken during these interviews, and each interview was summarized afterwards. The summaries were checked by the interviewees, and fed back to the project team. At the end of the second year of implementation, interviews were held separately with each school coordinator $(n=4)$, PE coordinator $(n=4)$, school health promoter $(n=4)$, and the project leader $(n=1)$. Topics explored included the HPSF process of development and implementation, factors influencing this process (coordination, team cohesion, bottom-up involvement, external support, and momentum-effect), adaptations in the school context as a response to HPSF, and the 
extent to which HPSF was integrated in the school after two years. These interviews were audiotaped, transcribed verbatim, and member-checked.

\section{Observations}

A researcher participated, observed, and took notes in the four schools and during all meetings of the project team and meetings of the health promoters. The aim was to learn about school dynamics, and to see and hear factors influencing the implementation process (rather than as a form of fidelity assessment). To create an open view, no observational checklist was used by the researcher. During school visits, the researcher randomly talked to school staff and children to hear about their experiences and perceptions regarding HPSF. Observations took at least one full week each year during effect measurements and regular visits (at least once every three months) to each school during the year. Notes were taken during and immediately after visiting the school.

\section{Barrier questionnaire}

The presence of perceived potential barriers for HPSF were collected by a 46-item questionnaire, distributed by e-mail, that all teachers and external PE were asked to complete digitally or by writing. The questionnaire was based on the Measurement Instrument for Determinants of Innovations (MIDI), a Dutch validated questionnaire developed by Fleuren et al. [71]. Items are formulated as a statement regarding barriers for HPSF related to the innovation, implementers, organization, or socio-political context. Responses to each statement ranged from 1 (totally disagree) to 10 (totally agree). Statements with an average score below 6 were defined as potential barriers. This corresponded to the grading system used in Dutch primary schools, which also uses a range from 1 to 10 for school tests, and scores below 6 as insufficient or fail. The questionnaire was completed once during the development year, and twice a year during the two years of implementation. To obtain data about dominating organizational issues, and factors influencing the process of development and implementation of HPSF, the questionnaire included open questions, e.g., 'Which five factors are in your opinion important to make HPSF successful?'.

\section{Practices questionnaire}

A questionnaire, based on and used in previous work by Gevers et al. [98] and O'Connor et al. [80], was used annually at the beginning of the academic year to assess nutritionand PA-related HP practices of teachers and parents, such as modelling behaviour and encouragement. All teachers received the questionnaire whereas parents only received it when they had signed the consent form (68\%). The paper-based teacher questionnaire consisted of 30 items; the digital parent questionnaire consisted of 23 items. Each item described a practice by using a statement, followed by some examples. Participants responded on a Likert scale from 1 (completely disagree) to 5 (completely agree). 


\section{Minutes of meetings}

Minutes were collected of the meetings of the project team, the health promoters, the working groups with parents and teachers, and the children's voice groups. Data derived from these minutes provided qualitative, in-depth information about the development and implementation of HPSF in each school and any experienced influencing factors.

\section{Analyses}

Thematic analyses were conducted of the qualitative data from the interviews, observations, and minutes [99]. Data were coded into themes based on the programme theory using NVivo (version 11.0). During this coding process, themes were reviewed several times to see if they still worked in relation to the data. After all data were coded, subcategories were created per theme if necessary, and when possible a distinction between inhibiting and promoting was made for the influencing factors. Next, the coded text was retrieved to create an overview per theme (or per subcategory) with the findings split up into the four schools to study similarities and differences. Furthermore, for each school, the frequency of similar answers to the open questions of the barrier questionnaire was calculated. Quantitative questionnaire data were analysed using SPSS (version 23). For each time of measurement and separately for each actor, descriptives were calculated per practice (teachers, parents) or potential barrier (teachers, external PE). Standardized effect sizes (Cohen's d) per school, defined as (mean at follow-up time of measurement minus mean at baseline) divided by standard deviation (SD) at baseline, were calculated for the practices. This effect size calculation was presented on top of the pre- and post-mean (SD) per school, to give an indication of the extent of the changes over time and be able to compare them between the schools. Only the teachers/parents who filled in both the questionnaire at baseline and at T1/T2 were included in this calculation. The effect sizes were categorized in accordance with Lipsey's guidelines [100]: small (0-0.32), medium (0.33-0.55), and large effect (>0.56). 


\section{Results}

\section{The pre-existing context of the four schools}

School days lasted from 8.30am to approximately 3.00pm on Monday to Friday, except for Wednesday, when schools finished around 12.30pm (Table 2). The schools had a 15minute morning break when children went outside for free play and ate their own brought morning snack. Lunch break time varied between 30 and 60 minutes across schools: 15 minutes' lunch, when they could eat their own brought sandwiches, and 1545 minutes of free play outside after lunch. These routines were comparable to other primary schools in the Netherlands. Physical education classes consisted of approximately $60 \mathrm{~min} /$ week, except for School 3 (S3), which had $120 \mathrm{~min} /$ week. All schools had a sports hall on-site or within walking distance and had several PA possibilities in the schoolyard and the neighbourhood. All schools, except S3, had limited HP policy and education.

The teacher practices questionnaire prior to HPSF was completed by $96 \%$ of all teachers (S1: 100\%; S2: 100\%; S3: 75\%; S4: 100\%). Some of most prevalent nutritionrelated practices of teachers prior to HPSF were encouraging the children to eat healthy foods (mean scores between 4.3 - 4.7), which was especially high in School 1 (S1), S3, and School 4 (S4) (Additional file 1). In S1 and School 2 (S2) the nutrition-related practice of teachers that was also much prevalent was adhering to school's nutritionrelated policy, whereby both schools had a mean score of 4.5. Moreover, also most prevalent in S2 was having clear healthy routines/habits (mean score (SD): $4.3(0.98)$ ), in S3 educating children on nutrition (mean score (SD): $4.6(0.70)$ ) and in S4 involving children in healthy nutrition (mean score (SD): 4.3 (1.03)). Some of most prevalent PArelated practices of teachers was creating sufficient access to PA (mean scores between 4.2 - 4.6), which was especially high in S2, S3 and S4 (Additional file 1). In S3 and S4 the PA-related practice of teachers that was also much prevalent was educating children about PA (mean scores (SD) in S3: 4.7 (0.68); and in S4: 4.5 (0.80)). Moreover, also most prevalent in S1 was having PA-friendly equipment available (mean score (SD): 4.4 (1.03)) and in S2 encouraging children to become physically active (mean score (SD): 4.5 (0.64)). The parental practices questionnaire prior to HPSF was completed by $66 \%$ of all the parents who had filled in the consent form (S1: 76\%; S2: 56\%; S3: 60\%; S4: 67\%). Most prevalent HP practices of parents at home were similar in all schools: making healthy foods available (mean score between $4.3-4.5$ ) and encouraging their child to eat healthy foods (mean score between 4.3 - 4.5), having PA-friendly equipment available (mean score between $4.2-4.4$ ), and encouraging their child to become physically active (mean score between $4.2-4.3$ ) (Additional file 1).

Data from the barrier questionnaire revealed that main potential barriers prior to HPSF, generally perceived by external PE, were a lack of time required for implementation (teachers ( $T$ ): mean score between 4.9 - 6.9; PE: mean score between 
4.6 - 8.4), limited training opportunities ( $T$ : mean score between 6.8 - 7.9; PE: mean score between $4.5-7.7$ ), and limited available personnel ( $T$ : mean score between $6.4-$ 7.5; PE: mean score between 4.0 - 7.1) (Additional file 2).

In addition, each school had their own specific situation. S1 (26 teachers, 324 children) was a merger of two separate schools, both of which were faced with declining numbers of children. The two schools moved to a new building at the start of HPSF (November 2015). Even though the merger created more work and a distracted focus, it also provided a natural opportunity to make a new start. S2 was also undergoing a merger process, planned for September 2016. For this merger the school building had to be renovated, so they had to move to a temporary location with limited PA possibilities in and around the school from November 2015 to September 2016. Before the merger, the school consisted of 15 teachers and 234 children; after the merger in September 2016, there were 23 teachers and 347 children. S3 (16 teachers, 233 children) had to deal with a major staff turnover at the start of HPSF. It had been participating in several other projects: 1 ) the Active Living project (prior to HPSF), in which they had changed their schoolyard to improve PA possibilities [70], 2) the JOGG (Youth on Healthy Weight) initiative, in which they had changed their school's water policy and provided free water bottles for all children, and 3) a project of RiskCare, a local private obesity prevention organization, in which they received support for training teachers to educate healthy lifestyle lessons. S4 (21 teachers, 389 children) joined HPSF later than the other three schools, i.e., at the end of academic year 2014/15. The school had been participating in the project of EU fruit, in which the school received fruit for all children twice a week. 
Table 2. HP elements in the four schools

\begin{tabular}{|c|c|c|c|c|}
\hline & School 1 & School 2 & School 3 & School 4 \\
\hline $\begin{array}{l}\text { School } \\
\text { routine }\end{array}$ & $\begin{array}{l}\text { Prior to HPSF: } \\
\text { - Lunch break time: } \\
\text { 45min } \\
\text { - Children bring their } \\
\text { own packed lunch. } \\
\text { Implemented in Y1: } \\
\text { - Provided healthy } \\
\text { lunch and mid- } \\
\text { morning snack } \\
\text { - Structured PA and } \\
\text { cultural sessions } \\
\text { during lunch break } \\
\text { - Increased lunch } \\
\text { break time to } \\
\text { 105min. }\end{array}$ & $\begin{array}{l}\text { Prior to HPSF: } \\
\text { - Lunch break time: } \\
\text { 30min } \\
\text { - Children bring their } \\
\text { own packed lunch. } \\
\text { Implemented in Y1: } \\
\text { - Provided healthy } \\
\text { lunch and mid- } \\
\text { morning snack } \\
\text { - Structured PA and } \\
\text { cultural sessions } \\
\text { during lunch break } \\
\text { - Increased lunch } \\
\text { break time to 95min. }\end{array}$ & $\begin{array}{l}\text { Prior to HPSF: } \\
\text { - Lunch break time } \\
\text { 45min } \\
\text { - Children bring their } \\
\text { own packed lunch. } \\
\text { Implemented in Y1: } \\
\text { - Structured PA and } \\
\text { cultural sessions } \\
\text { during lunch break. }\end{array}$ & $\begin{array}{l}\text { Prior to HPSF: } \\
\text { - Lunch break time: } 1 \\
\text { hour } \\
\text { - Children bring their } \\
\text { own packed lunch } \\
\text { or go home for } \\
\text { lunch. } \\
\text { Implemented in Y1: } \\
\text { - Structured PA and } \\
\text { cultural sessions } \\
\text { during lunch break }\end{array}$ \\
\hline Policy & $\begin{array}{l}\text { Prior to HPSF: } \\
\text { - Limited to no HP } \\
\text { policy. } \\
\text { Implemented in Y1: } \\
\text { - Birthday treat } \\
\text { policy } \\
\text { - Water policy }\end{array}$ & $\begin{array}{l}\text { Prior to HPSF: } \\
\text { - Limited to no HP } \\
\text { policy. } \\
\text { Implemented in Y1: } \\
\text { - Water policy } \\
\text { Implemented in Y2: } \\
\text { - Birthday treat policy }\end{array}$ & $\begin{array}{l}\text { Prior to HPSF: } \\
\text { - Birthday treat policy } \\
\text { - Water policy }\end{array}$ & $\begin{array}{l}\text { Prior to HPSF: } \\
\text { - Limited to no HP } \\
\text { policy. }\end{array}$ \\
\hline Education & $\begin{array}{l}\text { Prior to HPSF: } \\
\text { - Limited to no HP } \\
\quad \text { education. } \\
\text { - PE classes once a } \\
\quad \text { week. } \\
\text { Implemented in Y2: } \\
\text { - Educational lunch }\end{array}$ & $\begin{array}{l}\text { Prior to HPSF: } \\
\text { - Limited to no HP } \\
\text { education. } \\
\text { - PE classes once a } \\
\text { week. } \\
\text { Implemented in Y1: } \\
\text { - Educational lunch } \\
\text { Development phase: } \\
\text { - Educational } \\
\text { programme on } \\
\text { healthy lifestyle. }\end{array}$ & $\begin{array}{l}\text { Prior to HPSF: } \\
\text { - Healthy lifestyle } \\
\text { education } \\
\text { programmes. } \\
\text { - PE classes twice a } \\
\text { week. }\end{array}$ & $\begin{array}{l}\text { Prior to HPSF: } \\
\text { - Limited to no HP } \\
\text { education. } \\
\text { - PE classes once a } \\
\text { week. }\end{array}$ \\
\hline Environment & $\begin{array}{l}\text { Prior to HPSF: } \\
\text { - Once a week fruit } \\
\text { from local } \\
\text { supermarket. } \\
\text { Implemented in } Y 1 \text { : } \\
\text { - Providing water } \\
\text { bottles. } \\
\text { Implemented in } Y 2 \text { : } \\
\text { - Vegetable garden } \\
\text { in neighbourhood. }\end{array}$ & $\begin{array}{l}\text { Prior to HPSF: } \\
\text { - } \\
\text { Implemented in Y1: } \\
\text { - Providing water } \\
\text { bottles. } \\
\text { Developmental phase: } \\
\text { - Vegetable garden }\end{array}$ & $\begin{array}{l}\text { Prior to HPSF: } \\
\text { - Active Living: PA- } \\
\text { friendly schoolyard. } \\
\text { _ JOGG: providing } \\
\text { water bottles. } \\
\text { - RiskCare: offered } \\
\text { health-promoting } \\
\text { programmes for } \\
\text { parents and children } \\
\text { and supported the } \\
\text { healthy lifestyle } \\
\text { education } \\
\text { programme. } \\
\text { Implemented in Y2: } \\
\text { - Vegetables in the } \\
\text { schoolyard. }\end{array}$ & $\begin{array}{l}\text { Prior to HPSF: } \\
\text { - EU-school-fruit: } \\
\text { Offered fruit twice } \\
\text { a week. }\end{array}$ \\
\hline
\end{tabular}




\section{Process of change}

Development and implementation of HPSF

Two top-down HP changes

Parental support for HPSF in S1 (89\%) and S2 (88\%) was high; they also had 100\% teacher support. S3 had no unanimous teacher support and 68\% parental support, mainly due to criticisms of the lunch. A fourth school dropped out because of a lack of bottom-up support. Due to these differences in support, HPSF was split up into two versions: 1) implementation of the provided lunch and the structured PA sessions, and 2) implementation of the structured PA sessions only. S1 and S2 continued with the first version of HPSF. Since the main criticism in S3 was on the lunch, this school decided to continue with only a focus on PA. After the withdrawal of the fourth school, another school from the same educational board was recruited. For this reason, this 'new' S4 did not go through a decision process with teachers and parents due to limited time as they joined the initiative at the end of the school year. They also focused only PA. In S1 and S2, the time for having lunch was increased to 20-30 minutes (Table 2). The caterer developed a lunch menu cycle that changed every ten weeks, in which at least $80 \%$ of the products met the advice of the Dutch Health Council [80]. A mid-morning snack, consisting of fruits and/or nuts, was also provided. The lunch, a bread-based cold meal, was typically Dutch. The PA sessions were carried out in the schoolyard and when available and needed, in parks, forest, and/or sports hall in the neighbourhood. All schools collaborated with sport clubs or other external partners to offer specific activities. The external PE of S1 and S2 were assigned to the same class for the whole year; the external PE of S3 and S4 were assigned to an activity. A sports and leisure organization supported the external PE during implementation when needed, and after a year they provided a training course ( 8 sessions of 2 hours) to supply them with additional tools on how to motivate children for active participation during the PA sessions.

\section{Additional HP changes}

Schools were informed about possible additional HP changes using a 'fruit basket' model designed by the researchers, which consists of a continuously expanding overview of available evidence-based structural HP changes [92]. Water bottles were provided to the children in S1, S2 and S4 (Table 2). S1 and S2 created a school water policy. $\$ 4$ gave the bottles to the children to take home and did not change their policy. S1 and S2 changed their school's policy on birthday treats. S2 implemented a once-aweek educational lunch. Due to limited structure, the health promoters developed short lessons for this educational lunch based on evidence-based educational healthy lifestyle programmes, which improved the content and structure. As a result of this, S1 also started to use the lessons. The school coordinators of S1, S2, and S3 decided to investigate possibilities for a vegetable garden in their schoolyard or neighbourhood. 
The S2 school coordinator aimed to start a HP educational programme in the next academic year (2017/18).

Influencing factors: interactions between HPSF and the school context

Coordination

According to the school coordinators, the main promoting factor to coordinate HPSF properly was support from and good collaboration and communication with the PE coordinator in the school (Table 3). The school coordinators also felt that having sufficient time during lunch breaks greatly improved the implementation process due to increased focus on their coordinating tasks. The S1 and S4 school coordinators noted that they were mainly busy with the daily practical issues around the lunch and structured PA sessions, which created a limited focus on the overall coordination of HPSF. This limited overall focus was perceived by the health promoters as inhibiting for the initiation of additional HP changes.

\section{Team cohesion}

According to teachers and external PE, important factors for success were the availability of external PE and the collaboration between PE and teachers (Additional file 3). However, particularly in the first year of implementation, the relationship and communication between external PE and teachers was suboptimal: they had to get used to each other, and their mutual responsibilities were not completely clear. Limited time available for formal meetings and limited permanent external PE impeded feedback opportunities and inhibited the process of creating good collaboration and communication. To improve this, all schools created one or more occasions for (in)formal contact to get to know each other and to create one team. Two schools (S1 and S2) had to deal with a merger during HPSF, which also increased the need for team cohesion. These schools had put extra efforts to create occasions for contact, by organising a party for everybody (S1) or introducing a training relevant for all teachers and PE (S2). Finally, PE coordinators indicated that external PE being assigned to a class promoted the cooperation with teachers and the relationship with children, while being assigned to an activity inhibited it.

\section{Bottom-up involvement}

To create sufficient support for implementation, it was perceived as important by the school coordinators to involve all actors immediately at the start of the decision and development process, especially parents who were critical of the HPSF approach. To build this involvement, the schools had started with an enthusiastic team of teachers: their positive attitude towards HPSF in formal and informal conversations with parents stimulated parents' enthusiasm, which created a positive atmosphere around HPSF and improved involvement. Teachers and external PE perceived bottom-up involvement and everyone's enthusiasm throughout the years as one of the main factors to make the 
two changes successful. However, the school coordinators also perceived that the involvement of teachers and parents to further improve school health promotion by additional HP changes faded after the two changes were successfully integrated in the school and had become part of the daily functioning of the school system. This was also seen in the responses to the barrier questionnaire: the majority of teachers reported that they could not fill out most of the statements, as they did not feel that it applied to them because they were not involved in implementing the two changes, which they considered the only components of HPSF. This result was also fed back to and discussed in the project team. According to the health promoters and project leader, this lack of perceived involvement was a key inhibiting factor to the implementation of additional HP changes.

\section{External support}

Support from external partners was highly appreciated and all schools indicated it as essential for the success of HPSF. Both the availability of external PE provided by childcare organizations and the practical support provided by a sports and leisure organization, the caterer, and the health promoters were considered being essential. Perceived promoting aspects for collaboration with external partners were regular feedback between the practical level of each school (the implementers) and the project team, direct communication with each other, and clear responsibilities of each person. The researcher's support was perceived as valuable when the provided feedback was to the point and tailored to each specific school. The coordinators perceived that the fruit basket model helped the schools to think of additional HP changes that are structural and evidence-based.

\section{Momentum}

Participants reported that implementation of the lunch in S1 and S2 was key in creating momentum to implement additional HP changes. The school coordinators of these schools indicated that the lunch made it easier to implement the water bottles because children did not have to bring any food or drinks to school anymore, and it created a good opportunity to change school policy around birthday treats. The health promoters indicated that the lunch made it also easier (compared to other schools in the region) to implement additional HP changes due to an improved health-promoting mind-set. This momentum effect was not observed in the four schools for the PA sessions. 
Table 3. Influencing factors on HPSF in the four schools

\begin{tabular}{|c|c|c|c|c|}
\hline & School 1 & School 2 & School 3 & School 4 \\
\hline Coordination & $\begin{array}{l}-/+ \\
\text { Mainly focus on } \\
\text { lunch break } \\
\text { changes, modest } \\
\text { collaboration } \\
\text { between school } \\
\text { coordinator and PE } \\
\text { coordinator. }\end{array}$ & $\begin{array}{l}++ \\
\text { Overall focus, optimal } \\
\text { collaboration } \\
\text { between school } \\
\text { coordinator and PE } \\
\text { coordinator. }\end{array}$ & $\begin{array}{l}\text { - } \\
\text { Limited time and } \\
\text { focus, limited } \\
\text { collaboration } \\
\text { between school } \\
\text { coordinator and PE } \\
\text { coordinator. }\end{array}$ & $\begin{array}{l}+ \\
\text { Mainly focus on } \\
\text { lunch break } \\
\text { changes, optimal } \\
\text { collaboration } \\
\text { between school } \\
\text { coordinator and PE } \\
\text { coordinator. }\end{array}$ \\
\hline Team cohesion & $\begin{array}{l}+ \\
\text { External PE assigned } \\
\text { to class, contact and } \\
\text { collaboration } \\
\text { improved over time, } \\
\text { also due to annual } \\
\text { party for whole } \\
\text { team. }\end{array}$ & $\begin{array}{l}++ \\
\text { External PE assigned } \\
\text { to class contact and } \\
\text { collaboration } \\
\text { improved over time, } \\
\text { also due to training } \\
\text { course for whole } \\
\text { team and much focus } \\
\text { and efforts from } \\
\text { coordinators. }\end{array}$ & $\begin{array}{l}\text { - } \\
\text { More classes than } \\
\text { external PE, external } \\
\text { PE divided by activity, } \\
\text { limited contact } \\
\text { between external PE } \\
\text { and teachers, meeting } \\
\text { helped to get to know } \\
\text { each other. }\end{array}$ & $\begin{array}{l}-/+ \\
\text { More classes than } \\
\text { external PE, } \\
\text { external PE divided } \\
\text { by activity, contact } \\
\text { between external } \\
\text { PE and teachers } \\
\text { when needed, } \\
\text { meeting helped to } \\
\text { get to know each } \\
\text { other. }\end{array}$ \\
\hline $\begin{array}{l}\text { Bottom-up } \\
\text { involvement: } \\
\text { development }\end{array}$ & $\begin{array}{l}++ \\
\text { Full year for } \\
\text { development, } \\
\text { teachers and } \\
\text { parents involved, } \\
\text { unanimous teacher } \\
\text { support, 89\% parent } \\
\text { support. }\end{array}$ & $\begin{array}{l}++ \\
\text { Full year for } \\
\text { development, } \\
\text { teachers and parents } \\
\text { involved, unanimous } \\
\text { teacher support, 88\% } \\
\text { parent support. }\end{array}$ & $\begin{array}{l}-/+ \\
\text { Full year for } \\
\text { development, } \\
\text { teachers and parents } \\
\text { involved, no full } \\
\text { teacher support, 68\% } \\
\text { parent support. }\end{array}$ & $\begin{array}{l}\text { Two months for } \\
\text { development, } \\
\text { teachers and } \\
\text { parents not fully } \\
\text { involved in } \\
\text { development } \\
\text { process. }\end{array}$ \\
\hline $\begin{array}{l}\text { Bottom-up } \\
\text { involvement: } \\
\text { implementation }\end{array}$ & $\begin{array}{l}\text {-/+ } \\
\text { Children voice } \\
\text { group, parental } \\
\text { volunteers, some } \\
\text { additional changes } \\
\text { with involvement of } \\
\text { parents and } \\
\text { teachers, teachers' } \\
\text { assumption that } \\
\text { HPSF consists only } \\
\text { of the lunch break } \\
\text { changes and does } \\
\text { not involve teacher } \\
\text { participation. }\end{array}$ & $\begin{array}{l}\text {-/+ } \\
\text { Children voice group, } \\
\text { parental volunteers, } \\
\text { some additional } \\
\text { changes with } \\
\text { involvement of } \\
\text { parents and teachers, } \\
\text { teachers' assumption } \\
\text { that HPSF consists } \\
\text { only of the lunch } \\
\text { break changes and } \\
\text { does not involve } \\
\text { teacher participation. }\end{array}$ & $\begin{array}{l}\text { - } \\
\text { Children voice group, } \\
\text { no parental } \\
\text { volunteers, teachers' } \\
\text { and parents' } \\
\text { involvement limited, } \\
\text { teachers' assumption } \\
\text { that HPSF consists } \\
\text { only of the lunch } \\
\text { break changes and } \\
\text { does not involve } \\
\text { teacher participation. }\end{array}$ & $\begin{array}{l}\text { - } \\
\text { Children voice } \\
\text { group, parental } \\
\text { volunteers, } \\
\text { teachers' } \\
\text { assumption that } \\
\text { HPSF consists only } \\
\text { of the lunch break } \\
\text { changes and does } \\
\text { not involve } \\
\text { teacher } \\
\text { participation. }\end{array}$ \\
\hline External & ++ & ++ & ++ & ++ \\
\hline support & $\begin{array}{l}\text { Many different } \\
\text { external partners } \\
\text { involved and } \\
\text { supporting the } \\
\text { schools in all aspects } \\
\text { of HPSF. }\end{array}$ & $\begin{array}{l}\text { Many different } \\
\text { external partners } \\
\text { involved and } \\
\text { supporting the } \\
\text { schools in all aspects } \\
\text { of HPSF. }\end{array}$ & $\begin{array}{l}\text { Many different } \\
\text { external partners } \\
\text { involved and } \\
\text { supporting the } \\
\text { schools in all aspects } \\
\text { of HPSF. }\end{array}$ & $\begin{array}{l}\text { Many different } \\
\text { external partners } \\
\text { involved and } \\
\text { supporting the } \\
\text { schools in all } \\
\text { aspects of HPSF. }\end{array}$ \\
\hline
\end{tabular}




\section{Integration of HPSF in the school context and perceived impact}

During the two years of implementation, a decline was observed in the number of perceived potential barriers among both external PE and teachers in S1, S2, and S4. This seems to indicate more integration of HPSF into the schools. An opposite result was found in S3, where the number of perceived barriers indicated by external PE increased during the implementation period. Factors in S3 that continued to be perceived as barrier throughout almost all measurements were: perceived outcome importance (mean score of the different measurements between $3.0-5.6$ ), observability (mean scores between $3.5-5.9$ ), adaptability (mean scores between $3.7-7.0$ ), availability of materials (mean scores between $3.6-6.8$ ), and support of parents (mean scores between $3.0-3.7)$.

Looking at the perceived impact of HPSF, some similarities were found across the schools, mainly regarding perceptions of improved health behaviours of children and improved healthy practices of teachers. All schools described perceptions that since HPSF the children created and managed their own activities more easily during free play, they were less bored during recess time, and fewer conflicts happened, which contributed to a calmer environment. Fewer impacts were mentioned regarding the school's way of working to create change in the whole school system, as the main focus was on the two changes. Furthermore, interviewees from S1 and S2 reported that lunchtime had become a more socializing moment, children ate a wider variety of foods and became more open to trying unfamiliar products. Issues regarding children's dietary behaviours became clearer and were easier to discuss with parents.

Overall, teachers' practices changed in a more favourable direction (Additional file 1). Large effect sizes were found for nutrition-related practices in S1 and S2, e.g., discussing (S1: effect size (ES)=0.07; S2: ES=0.81) and educating about nutrition (S1: $E S=0.38, S 2: E S=0.91$ ), and monitoring children's dietary behaviours (S1: $E S=1.16, S 2$ : $E S=0.09$ ). In S1 and S4 large effect sizes were found for teachers' PA-related practices, such as involving children in PA (S1: $E S=0.85, S 4: E S=0.59)$, and having routines/habits for PA (S1: ES=0.86, S4: ES=0.62). Teacher's modelling behaviour regarding nutrition and PA changed in $\mathrm{S} 1$ (nutrition: $E S=0.35, P A: E S=0.69$ ), S2 (nutrition: $E S=0.47, P A$ : $E S=0.36$ ), and $S 4$ (nutrition: $E S=-0.11, P A: E S=0.33$ ) mostly in a favourable direction, with often medium effect sizes. Effect sizes in S3 could not be determined due to a limited sample size as only four teachers filled out the questionnaire at both baseline and follow-up. Some parental practices changed, though none with a large effect size (Additional file 1). Medium effect sizes for parental practices were found for educating about PA (ES between $-0.05-0.36$ ) and emotional feeding (ES between $-0.33-0.14$ ), which mostly changed in a favourable direction; involving children in PA (ES between $0.33-0.04$ ) and making PA-stuff available (ES between $-0.48-0.00$ ) changed mostly in an unfavourable direction. 
In addition, school-specific perceived impacts for S1 include: the school team became closer, and a school day was seen more as an entirety that fits together. It was also perceived that the children became more creative, worked more together, and talked differently about healthy nutrition in school: it became a part of their identity and not just some school activity. In S2, the school coordinator indicated that teachers' focus on healthy behaviours had improved, e.g., teachers used more often healthy lifestyle topics in their lessons, they tended to keep each other updated regarding healthy lifestyle news items, and they were more aware of their own modelling behaviour. It was perceived in S3 that children became more enthusiastic about PA and going outside; teachers used more often healthy nutrition topics in their lessons. The interviewees of S4 indicated that teachers were more aware of possibilities for PA in school, and their interest in how to improve children's dietary behaviours had increased slightly.

\section{Discussion}

The current study explored the implementation of HPSF and the processes through which HPSF and the school context adapt to one another over time. Even though similarities existed since the schools are all part of the Dutch school system, the schools dealt with different contextual issues. These differences in context also influenced the evolution, implementation and impact of HPSF, demonstrating the importance of a contextual approach $[42,54]$.

Top-down advice and external practical support was perceived as helping the schools to initiate a positively disruptive change. Bottom-up involvement was needed throughout the process to contextualize and optimize changes and to create ownership. Sufficient coordination and communication at the school level, the availability of external PE, team cohesion, and feedback loops among all actors involved enhanced the implementation of the changes. These findings of the current study, in which we used a systems approach, are consistent with and add to the findings and recommendations of previous studies which also point to the importance of feedback, external support, clear coordination and communication, and bottom-up involvement for sufficient adoption and implementation of school health promotion programmes [20,95, 96, 101]. The current study further extends the knowledge by, among other things, insight on creating disruption in the schools. Modifying the school lunch acted as an entry point for health improvement action due to the particular Dutch context in which provision of lunch by schools is not typical practice [97], and appeared to act as a catalyst for additional HP changes. Most of the implemented additional HP changes were described as being facilitated by the provided lunch.

The PA sessions did not have this disruptive effect in the schools, also not in S1 and S2. Two explanations can be given for this. First, while the lunch acted as a highly visible 
change in practice with everyday implications for parents and teachers, the PA sessions did not appear to have such a visible impact on parents and teachers, it's perceived influence being primarily with the children themselves. Perhaps due to the more limited number of stakeholders impacted by this change, the PA sessions did also not lead to much discussion among the people involved. Second, the topic of the change could also be a reason. It was observed in the different data sources that changes related to nutrition seemed to come much closer to essential aspects of parenting than changes related to PA. Altogether, this seems to indicate that both the topic of the change and the impacts of the disruption across multiple stakeholder groups is important.

Adaptations in the school context also occurred in teachers' HP practices: in S1 and S2, teachers' practices changed after two years of HPSF, several with a large effect size. Interestingly, looking at the mean, SD and effect sizes of the modelling practices of teachers, only moderate improvements can be seen, even though during the interviews the schools indicated a specific focus on modelling [102]. However, since no statistical tests were conducted, no hard conclusions could be drawn and further analysing is needed. Furthermore, the findings showed that aspects of the health promoting school concept [19], such as creating a HP environment or participation of parents and children, were in the first two years of implementation often directly related to the two top-down changes. This means that even though several impacts on health behaviours were perceived, there is still room for improvement to further increase the impact on the whole school system. However, as also indicated in the programme theory, this system change takes time due to the feedback loops that need to develop in the system.

The main recommendations resulting from this study were related back to the programme theory and combined into five key learning points for research and practice (Table 4). Four learning points can hereby be seen as conditions that were successful in the participating schools to create a major change that should lead to disruption; the last learning point is related to how to use a created disruption. 
Table 4. Key learning points

How to create a disruption?

1. Creating a disruption in a school takes time and needs bottom-up involvement.

2. Regular contact among all actors is required to get to know each other and to manage expectations.

3. Top-down advice and external practical support are important for creating a disruption.

4. To contextualize and realize changes feedback loops are required among all involved actors.

This learning point shows the importance of bottom-up involvement as indicated in the programme theory. Moreover, it also relates back to the several loops of feedback arrows between HPSF and the school context. In the four participating schools was seen that creating bottom-up involvement immediately at the start of the developmental phase took time but seemed to increase people's ownership and support. Implementation of changes also took time as the school needed to find a new way of working in the school to create for example a good collaboration between the teachers and the external PE.

This learning point relates back to the importance of sufficient coordination and team cohesion. In the four participating schools was seen that regular contact between the people involved, not only to discuss the content, but also to get to know each other, helped to create more understanding and feelings of mutual support. Regular contact between teachers and external PE improved team cohesion in the school, which enhanced implementation. In particular, communication about expectations of everybody's responsibilities appeared to be important.

This learning point shows the importance of external support, as indicated in the programme theory. In the four participating schools was seen that top-down advice and practical support from external partners helped the schools by providing personnel, money, materials, and knowledge.

This fourth learning point does not only relate back to the several loops of feedback arrows in the programme theory between HPSF and the school context, it also shows the importance of external support and the involvement from bottom-up. In the four participating schools was seen that feedback loops in school among staff, children, and parents made a change better fit into the school context with its specific needs and wishes. Feedback loops between school and external partners made the external support to school, to realize the changes, as efficient as possible.

How to use a disruption?

5. A disruption is useful for implementing This last learning point relates back to the loop in the bottom of additional HP changes on the same topic. the programme theory which indicates the momentum-effect. In this study the provided lunch disrupted the existing dynamics in the school and created momentum for nutrition-related additional HP changes, as people perceived these additional HP changes as something that came along with the provided lunch. The health promoters felt that due to the lunch in S1 and S2, additional nutrition-related HP changes were implemented with less discussion and easier acceptance, compared to other schools in the region, due to an improved health-promoting mind-set. However, the lunch did not create momentum for not nutritionrelated initiatives, i.e., PA-related. 


\section{Strengths and limitations}

The results should be considered in light of the study's strengths and limitations. A strength of the study is that due to using mixed methods, we were able to employ the principle of data triangulation and combine the accuracy of quantitative questionnaires with in-depth insights afforded by interviews, observations, minutes, and open questions. Triangulation is a strategy that facilitates validation of data through crossverification from different sources [103], and is stimulated by other researchers to employ in process evaluations [88]. Using CARA meant that the researchers in this pilot were not external observers, but actively participating partners in the initiative. The researchers not only evaluated the processes of change by using mixed methods, but also supported the schools in their processes. Researchers' support in this pilot consisted of offering their knowledge and expertise and by providing regular feedback based on the results of the mixed methods. However, schools always decided themselves what to do with this information. The active participation of researchers helped the schools to improve their changes, and it gave the researchers a deep and honest insight into each school's process of change, as a relationship of trust was built up with the people in the school. However, due to this research approach, the researchers interfere with the implementation processes and are not fully objective anymore, which can be seen as limitation. By conducting the process evaluation prior to the effect evaluation, where a quasi-experimental study design was used, we were able to combine the best of two worlds: the advantages of a researcher involved in the process of change without knowing the effects, and studying the effects objectively by the quasi-experimental design [47].

Another limitation of the study is that it was impossible to fully assess and understand all aspects of each school's context and process of change due to limitations in time, resources, and participant burden [47]. To deal with this issue, we followed recent research suggestions to mainly focus on the factors that are indicated as relevant for improving school health promotion [21, 42, 68, 71]. Finally, the four pilot-schools could be classified as early adopters, who were open for system change. Scaling up the HPSF initiative should also include schools that are less open for change. Bottom-up involvement from the start is hereby crucial to create ownership and support in the school. Communicating about the benefits experienced by the early adopters could help to increase the engagement in these schools [104]. When scaling-up, the support provided by the researchers should be maintained to contribute to the process of feedback in the schools. This supporting role might be incorporated in the work of the health promoter who is connected to the school. 


\section{Conclusions}

Taking the studies' limitations and strengths into account, it can be concluded that creating an initial, highly visible and well supported positive disruption to improve school health can act as a catalyst for wider school health promotion efforts. Conditions to create a positive disruption are enough time, and sufficient bottom-up involvement, external support, team cohesion and coordination. The focus should be on each specific school, as each school has their own starting point and process of change. 


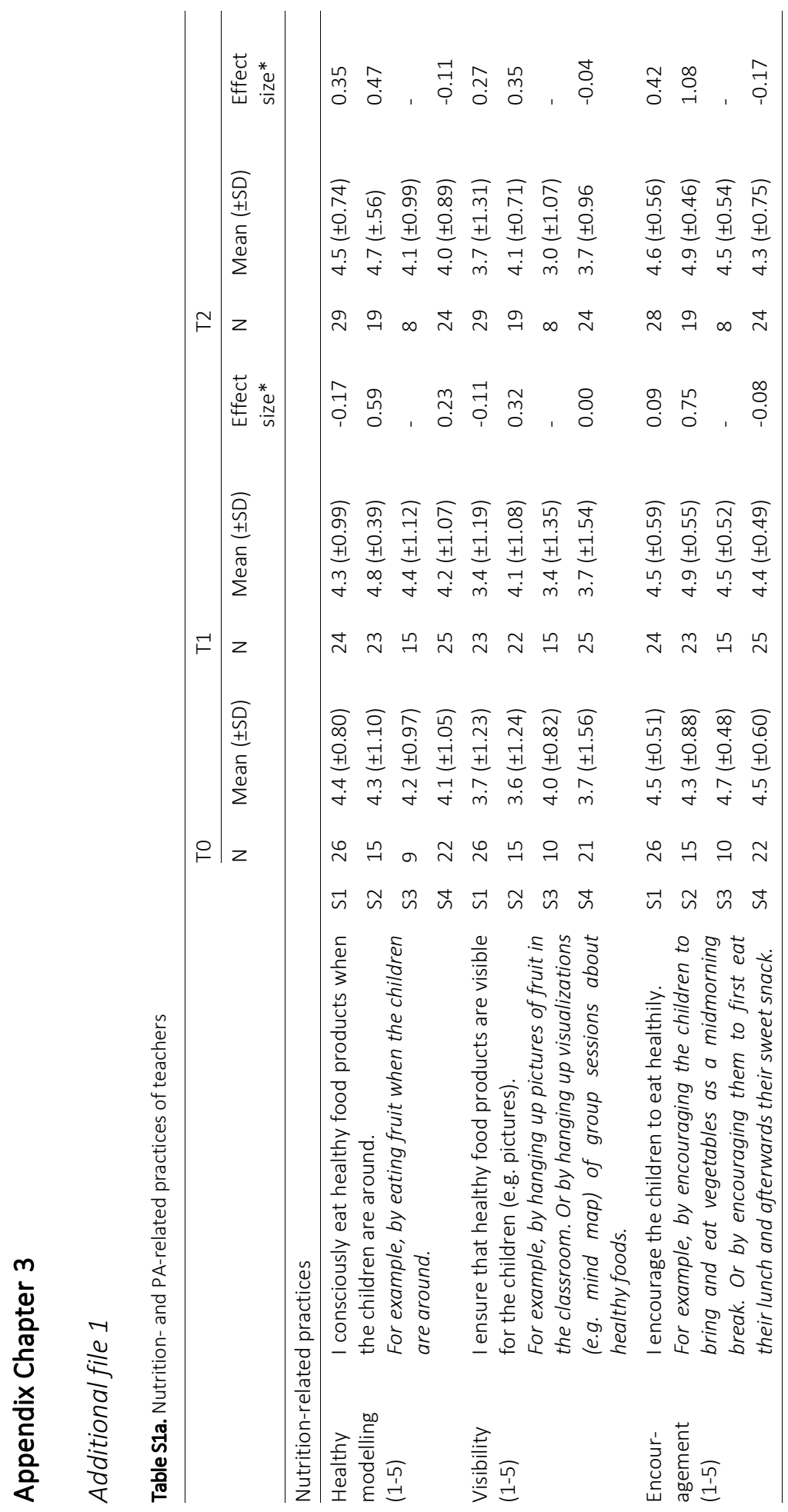




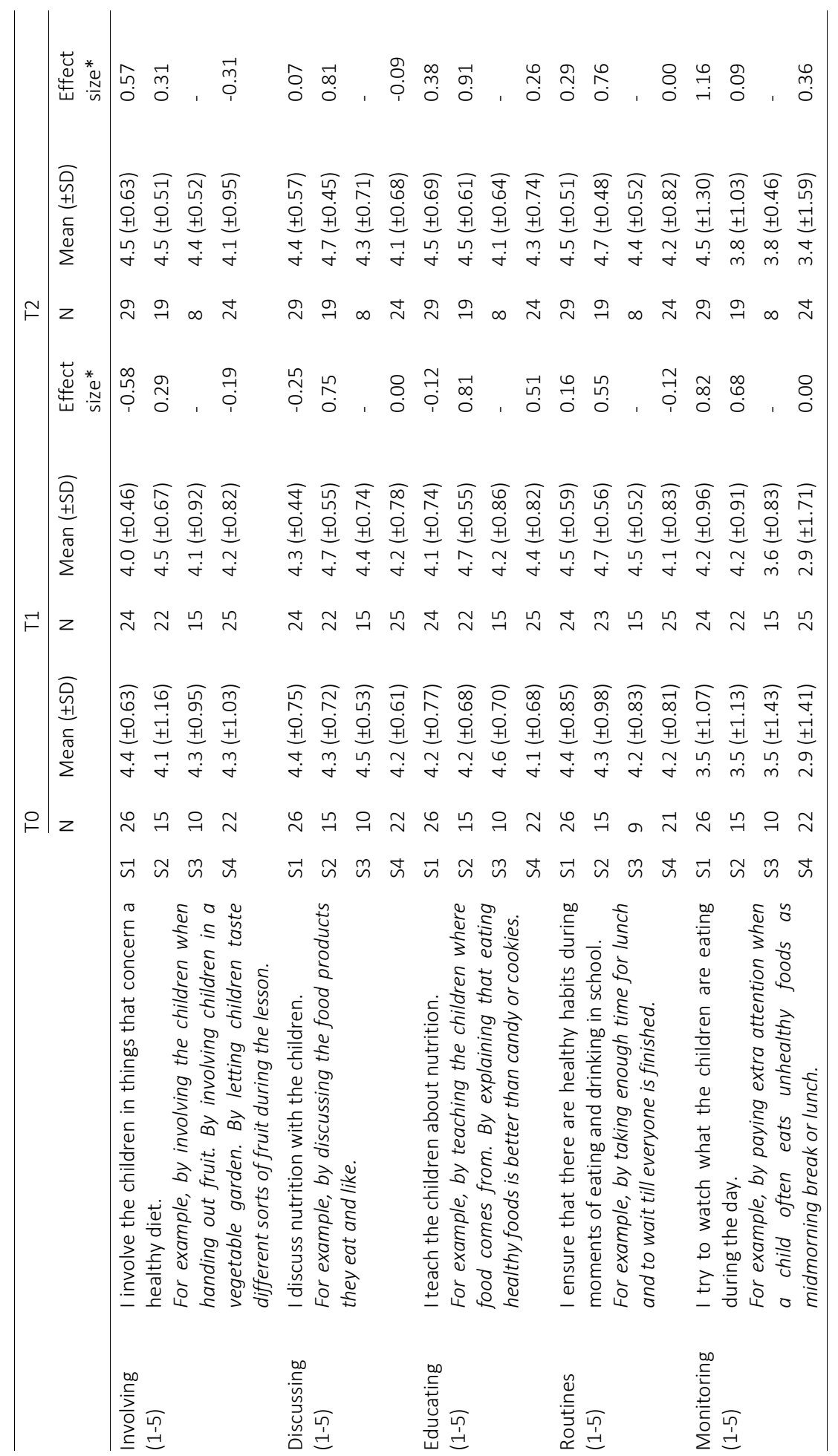




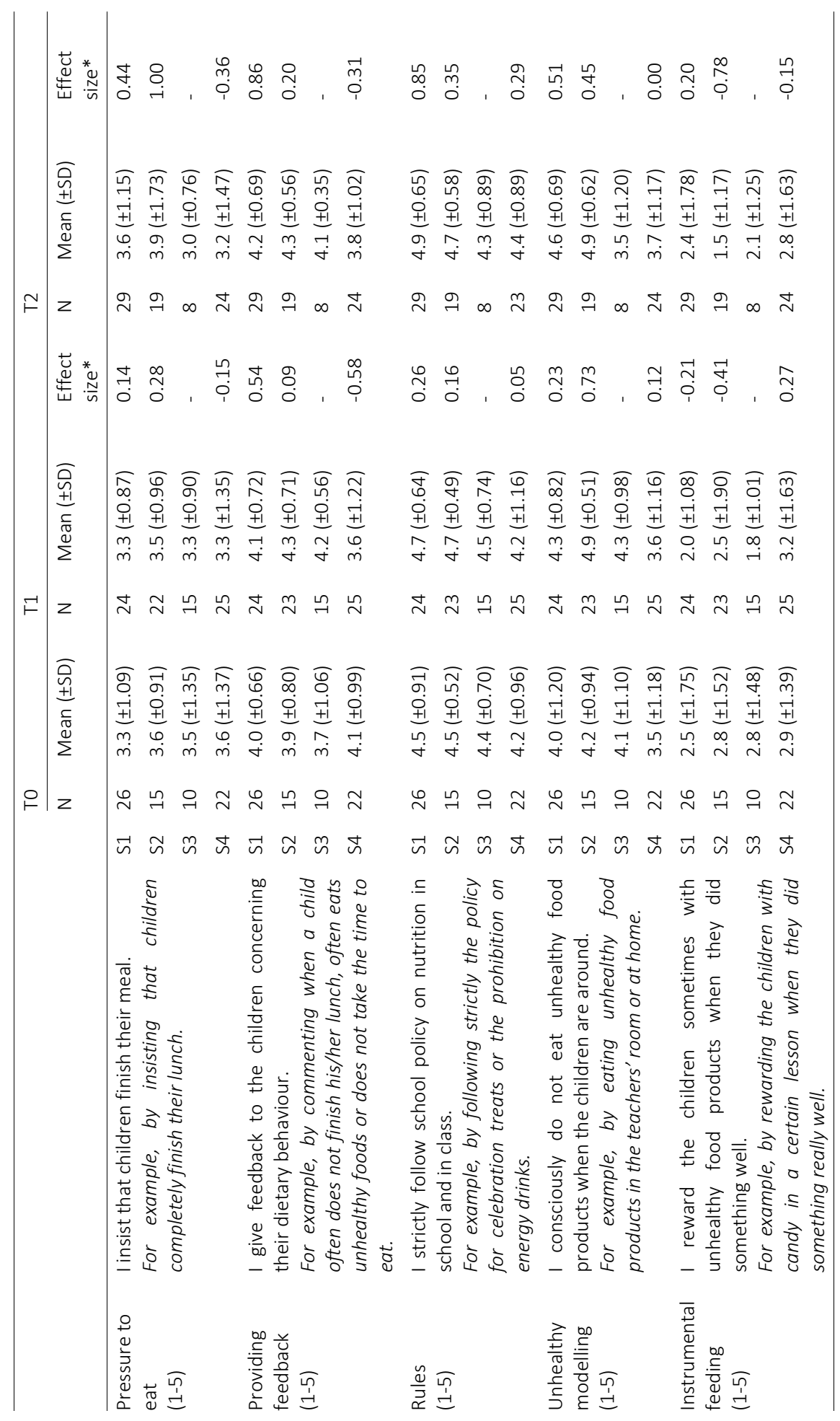




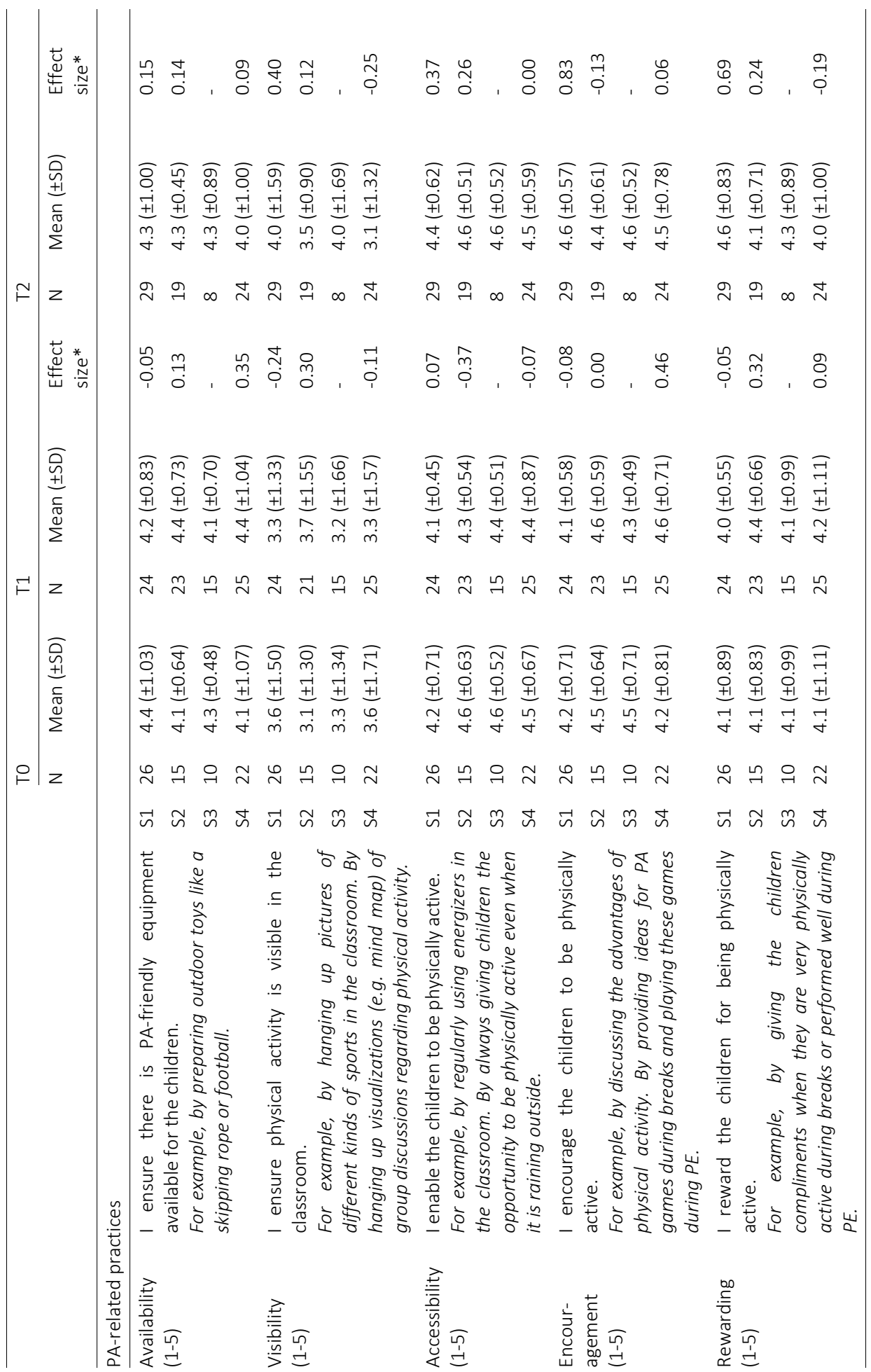




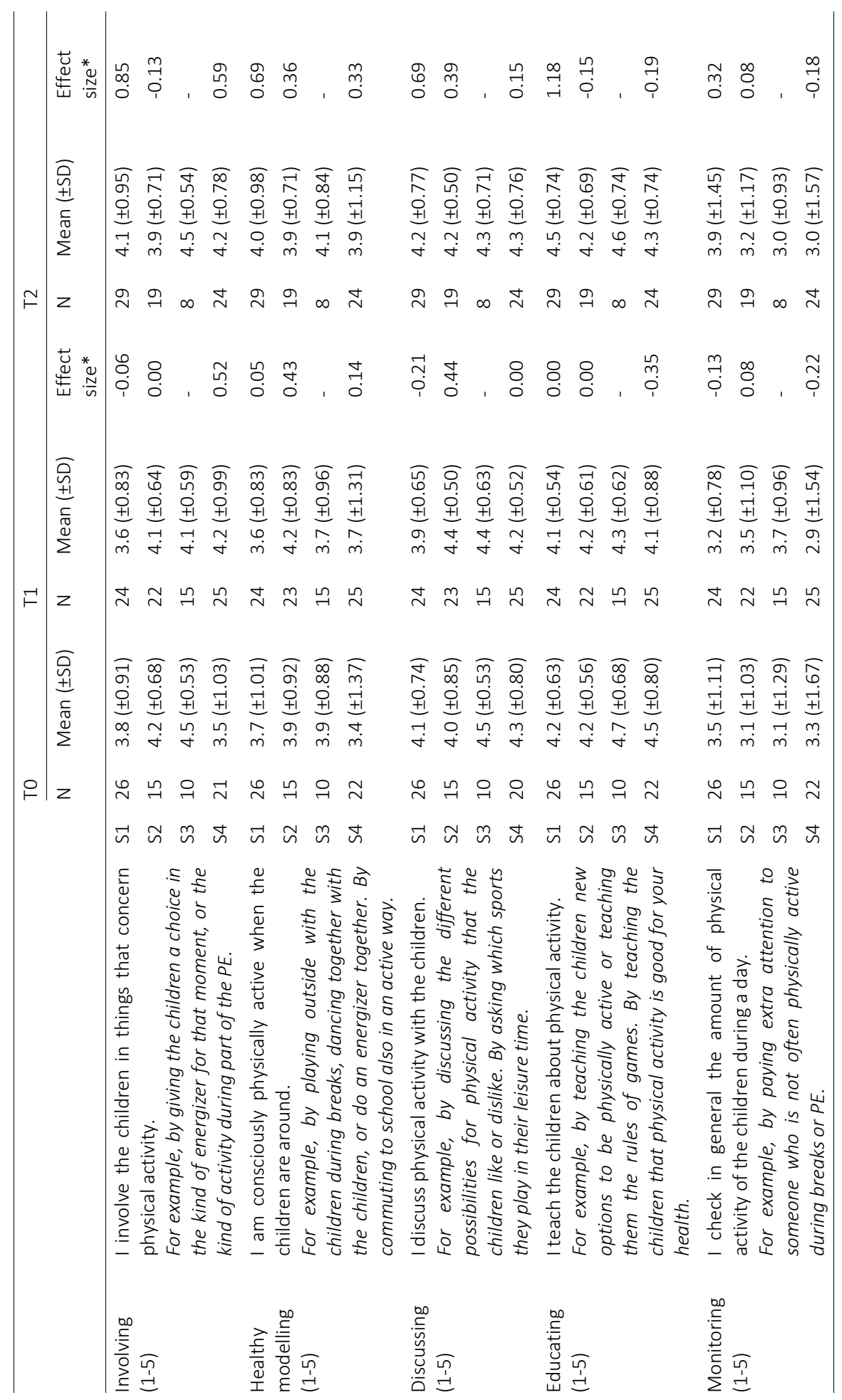




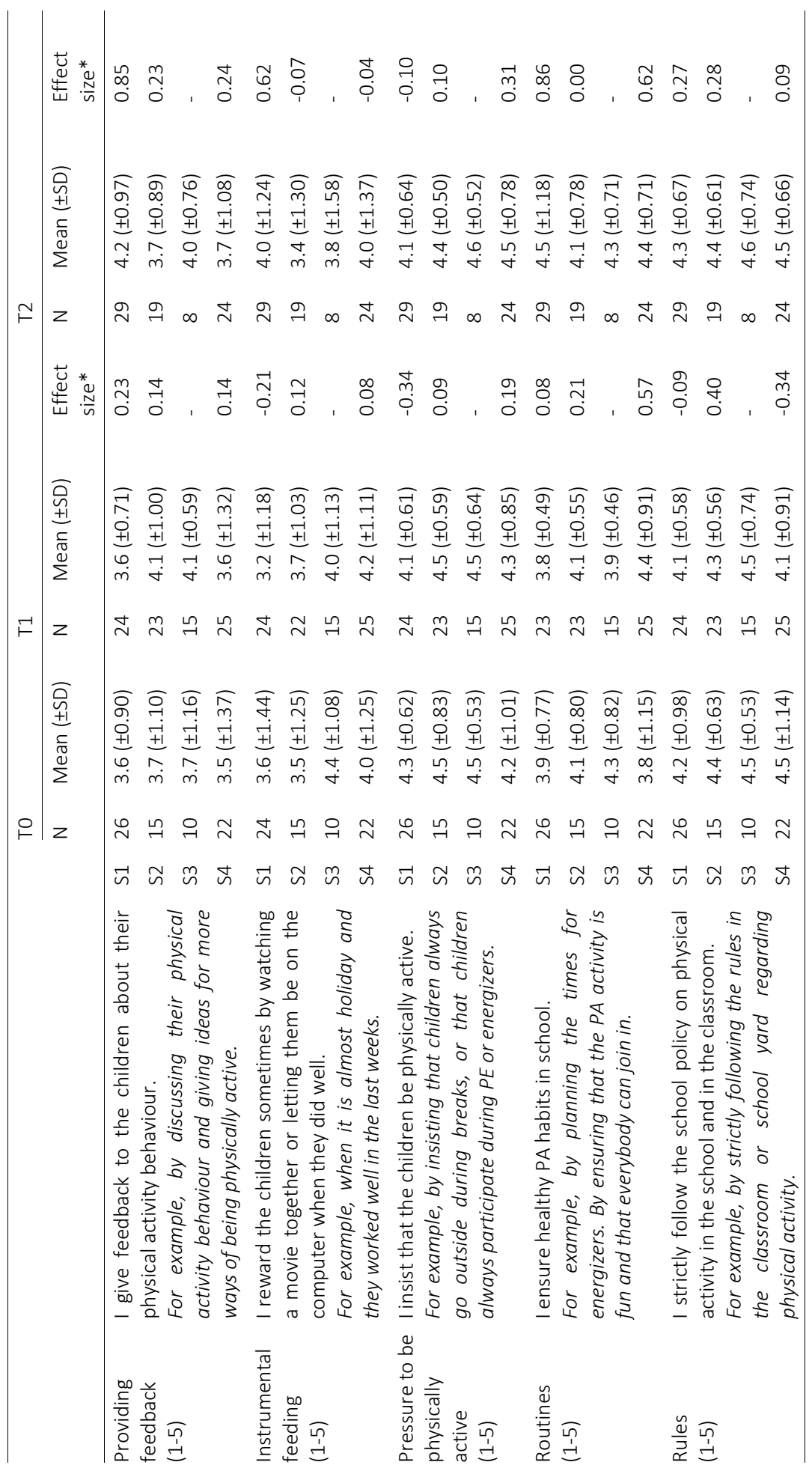


Chapter 3

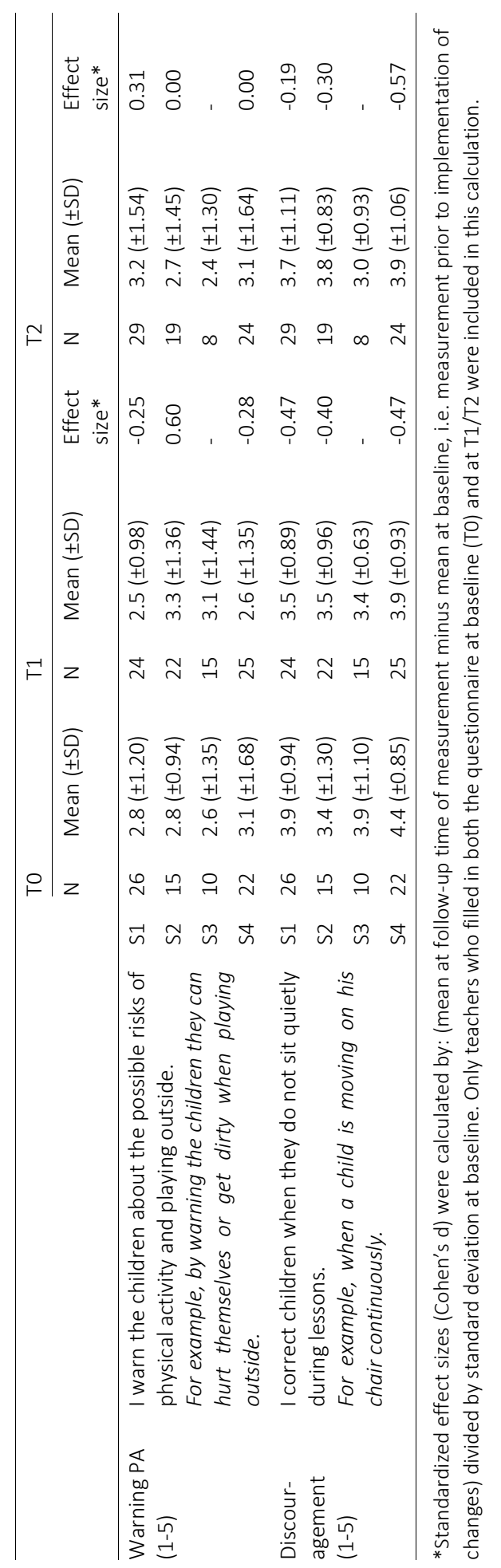




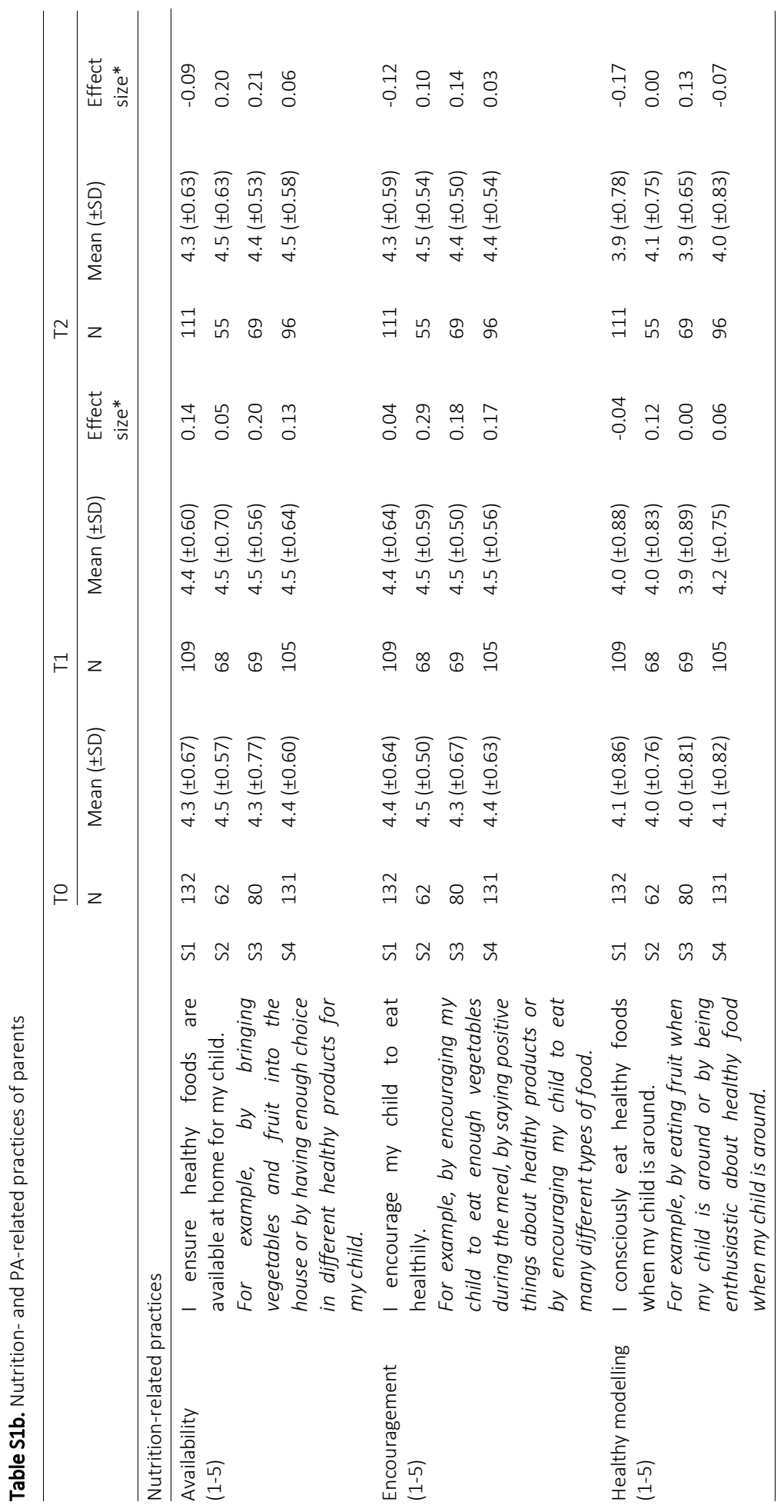




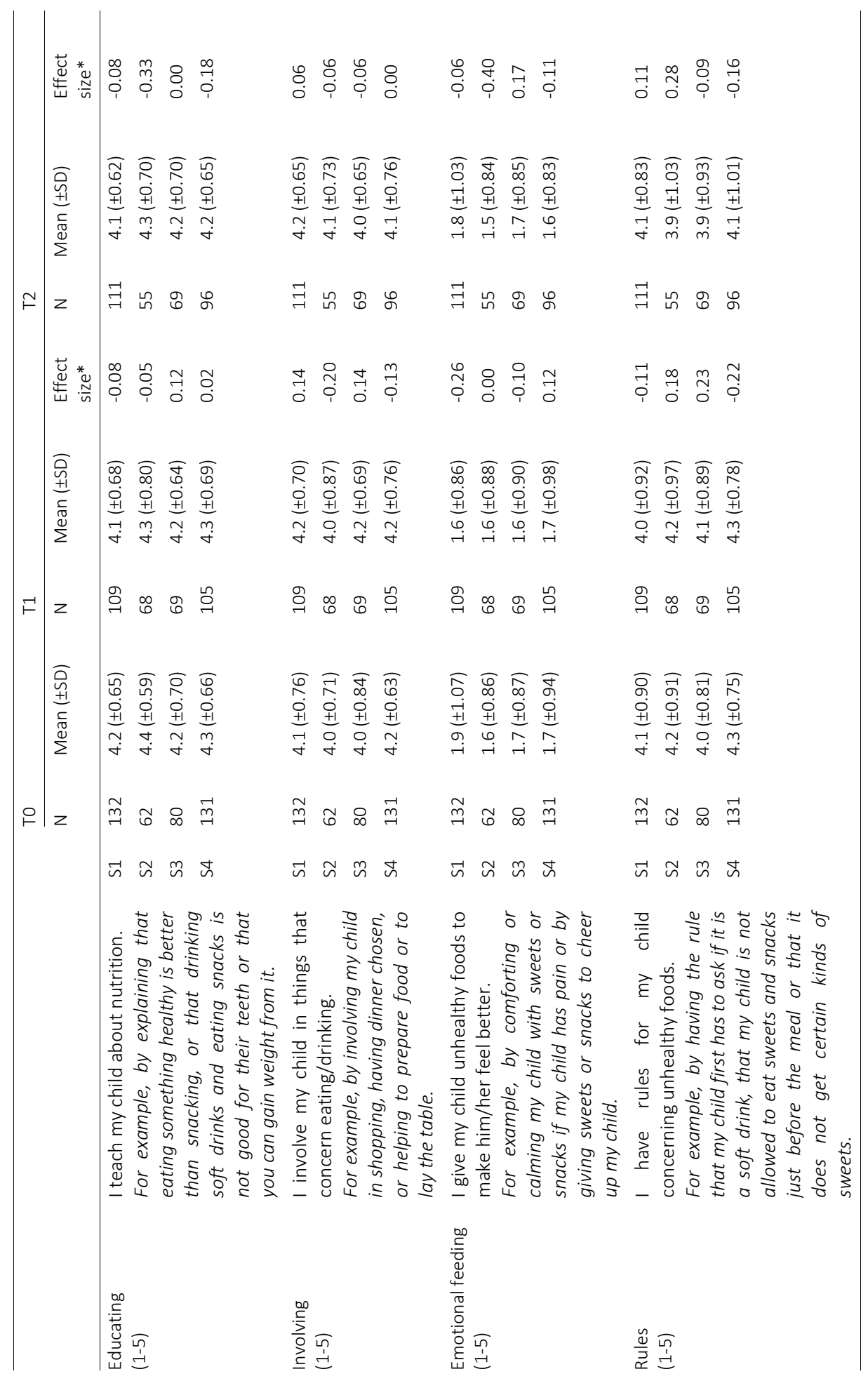




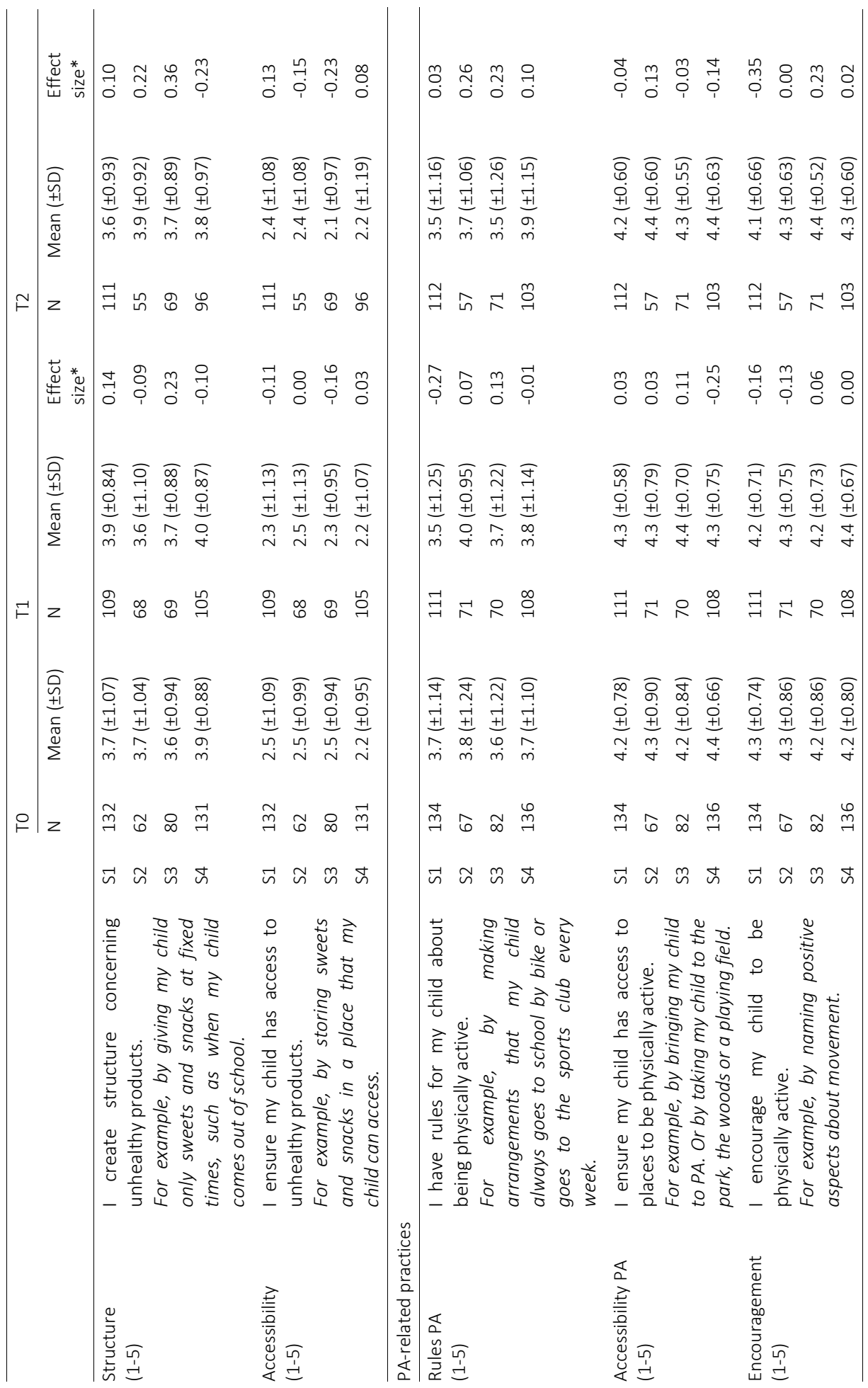




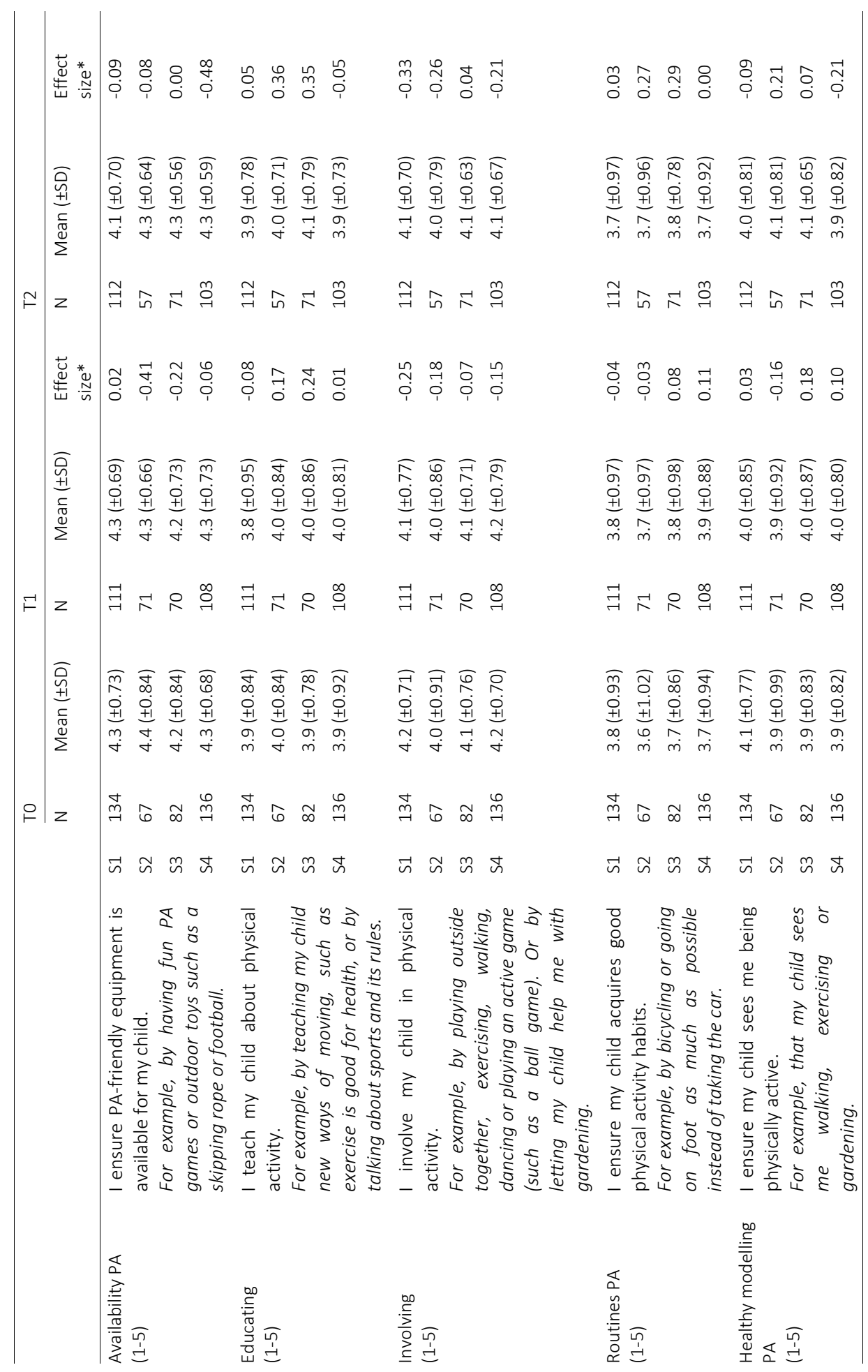




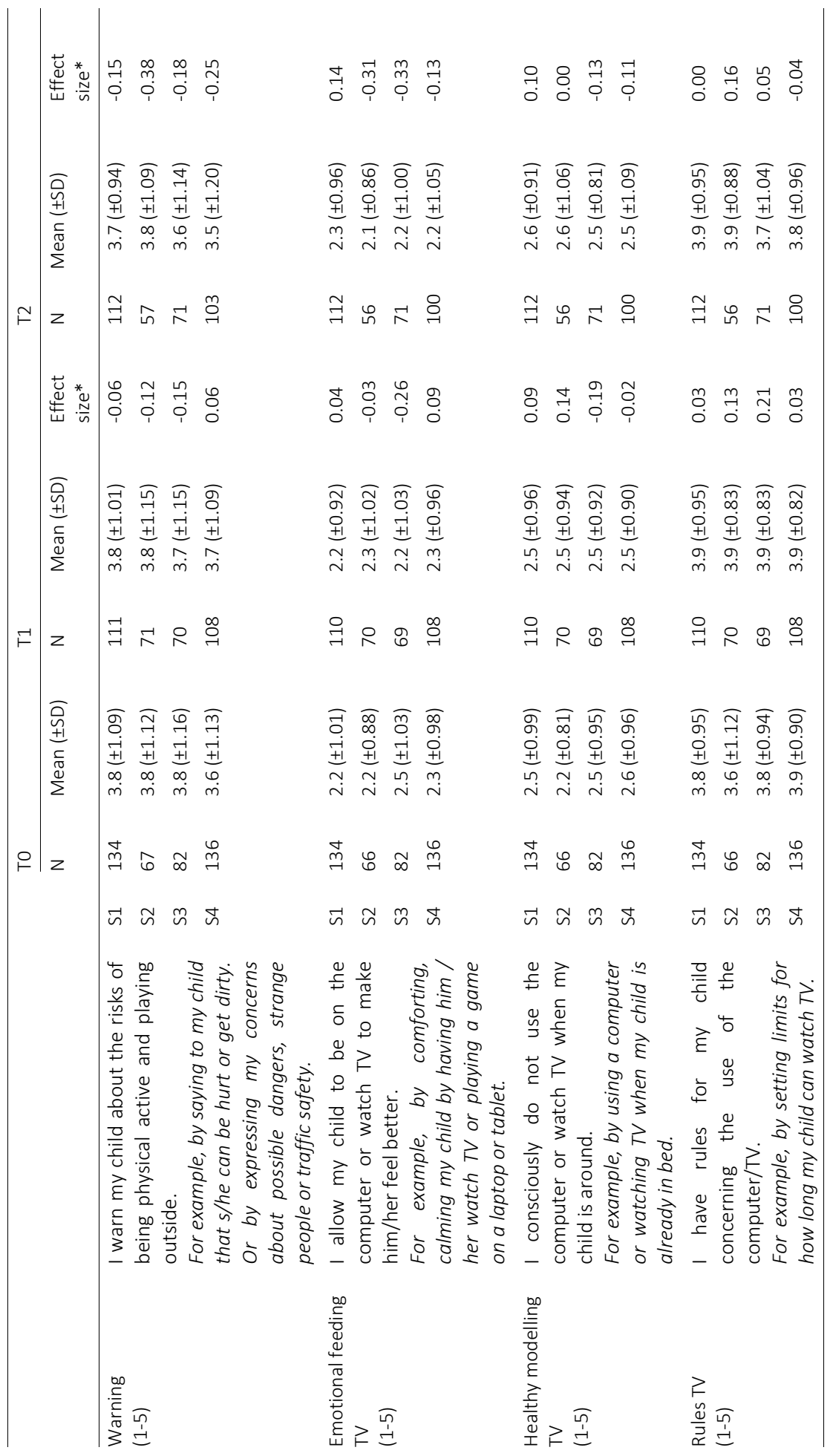




\section{Chapter 3}

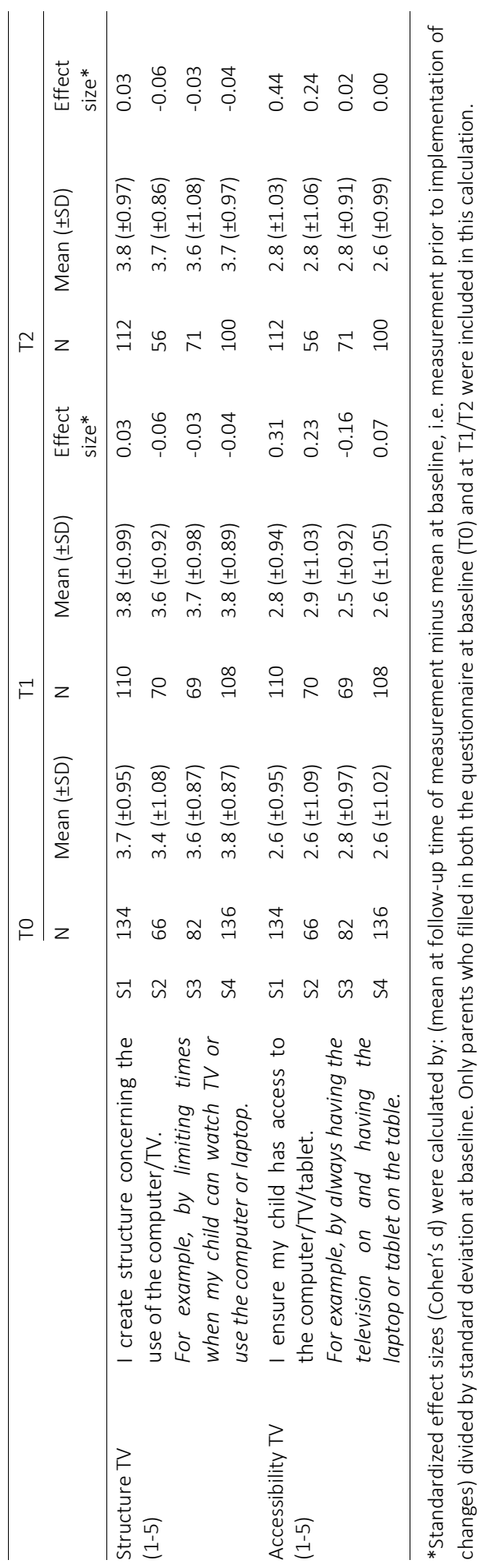




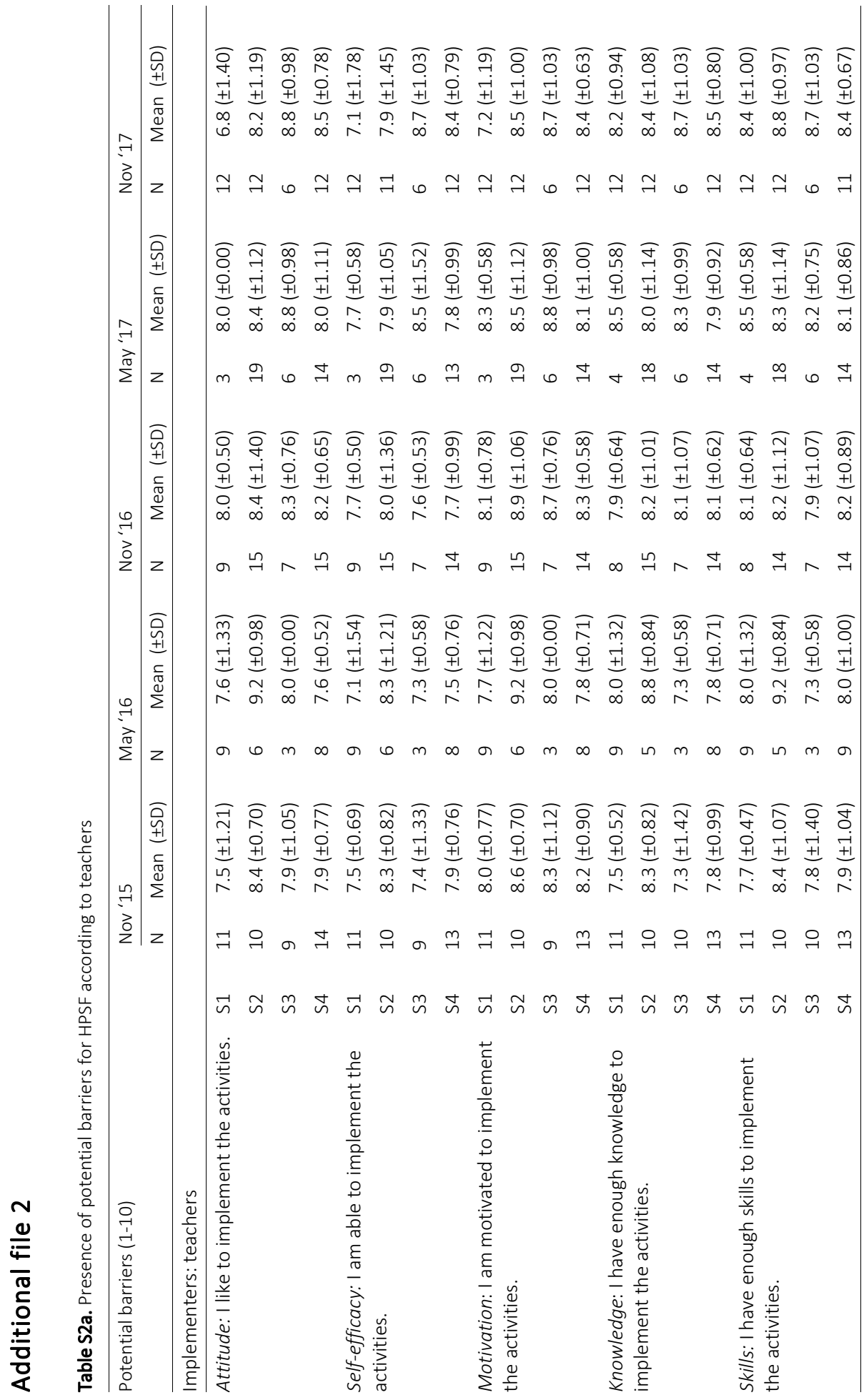




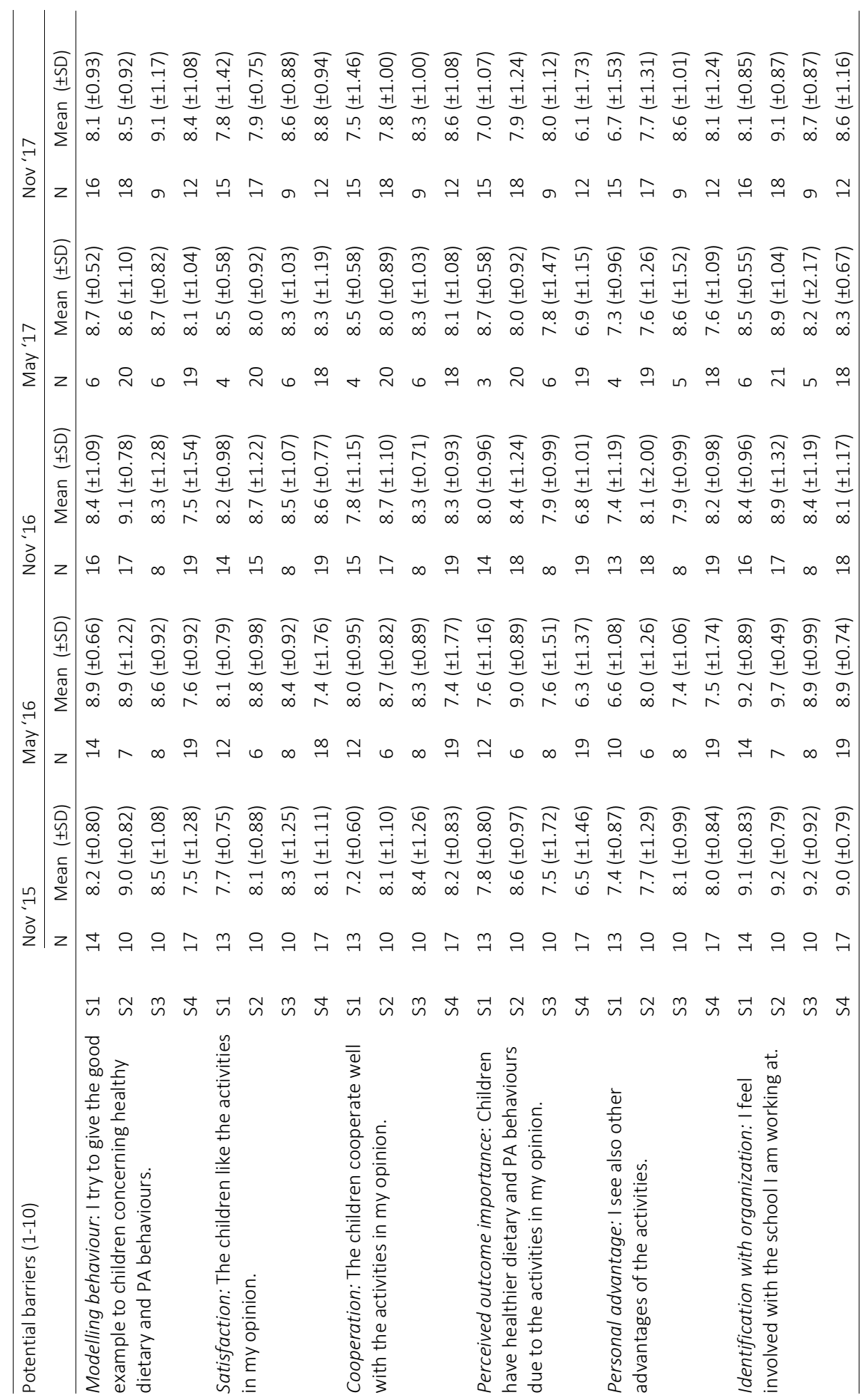




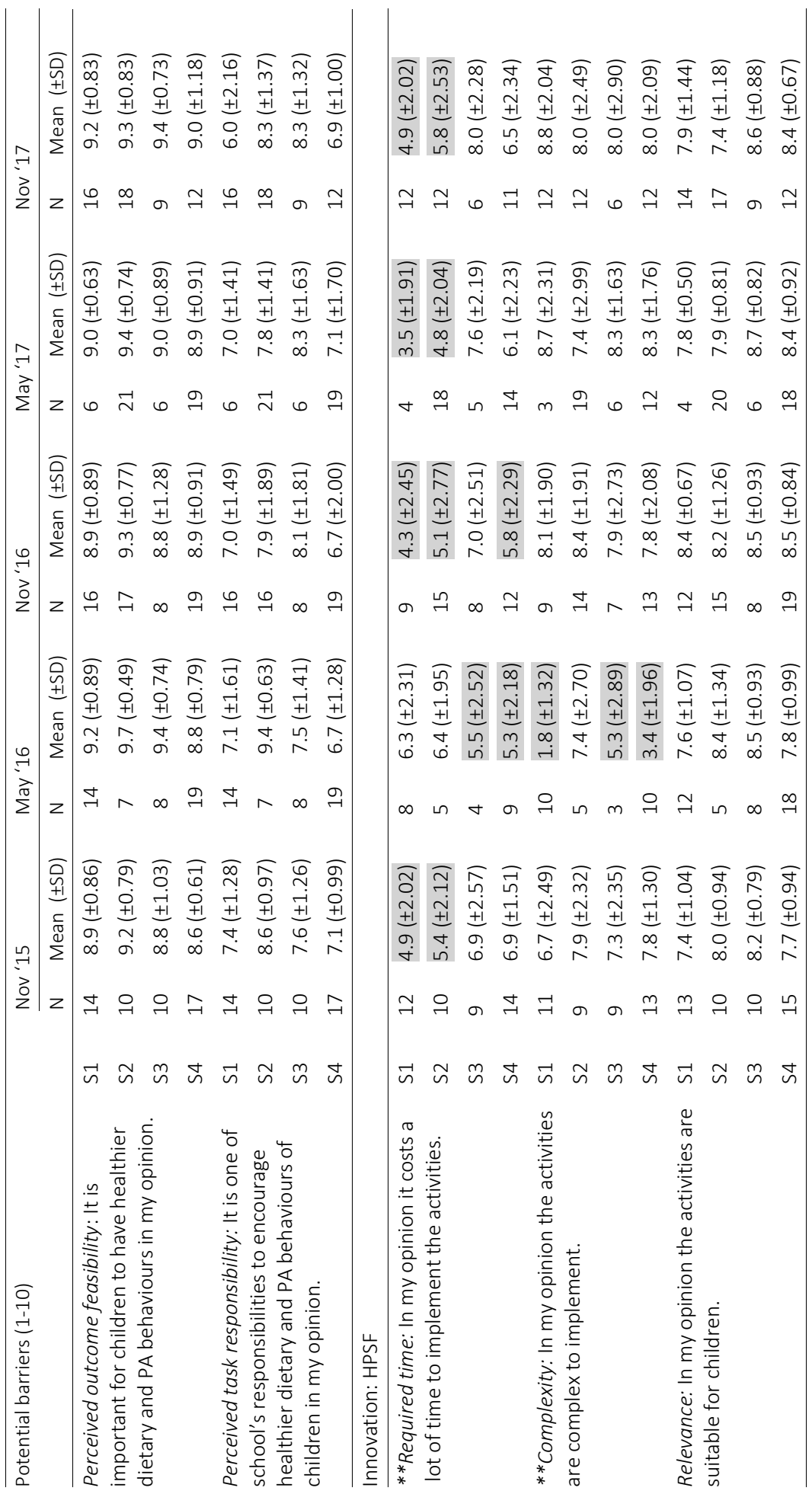




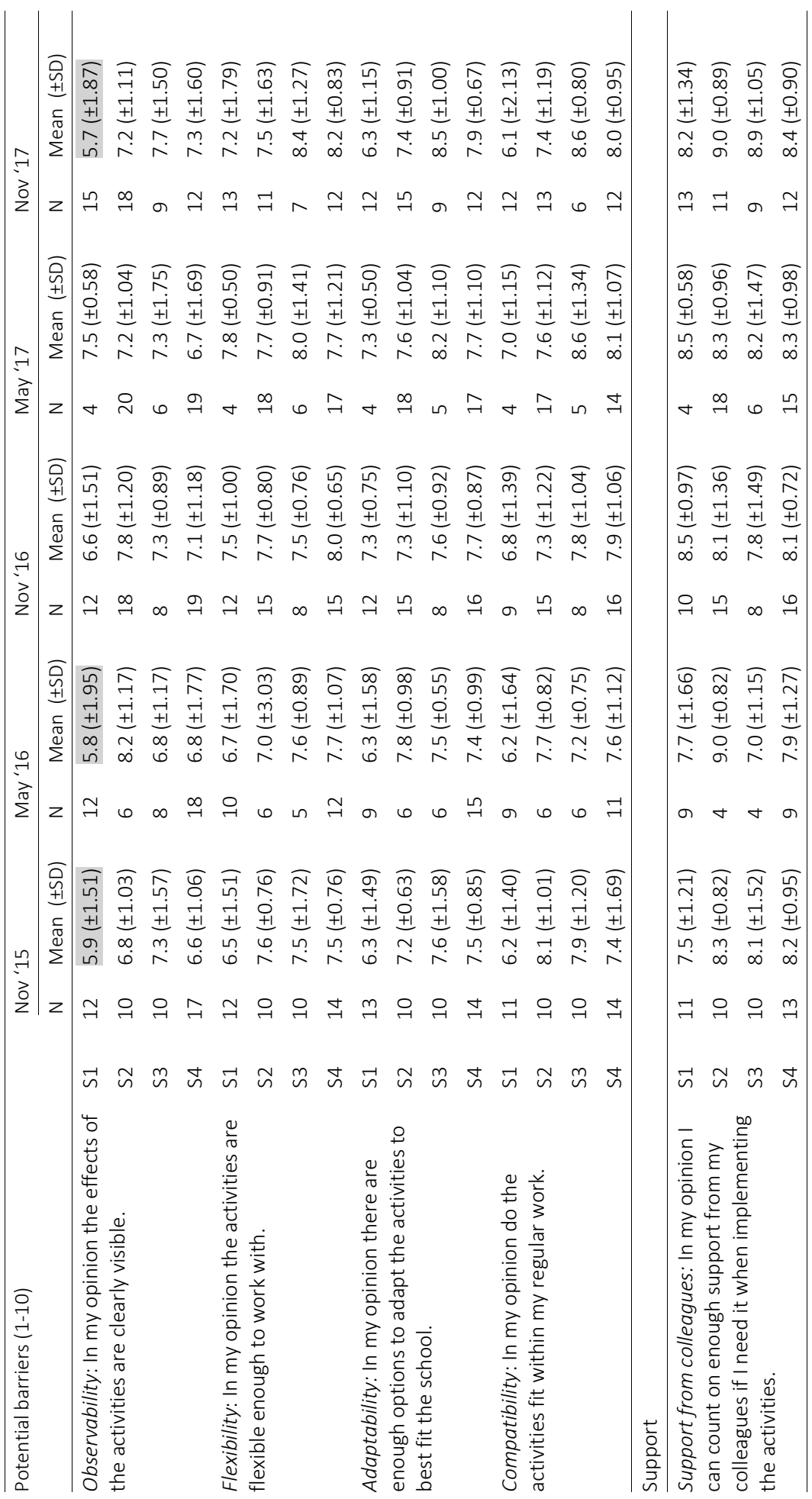




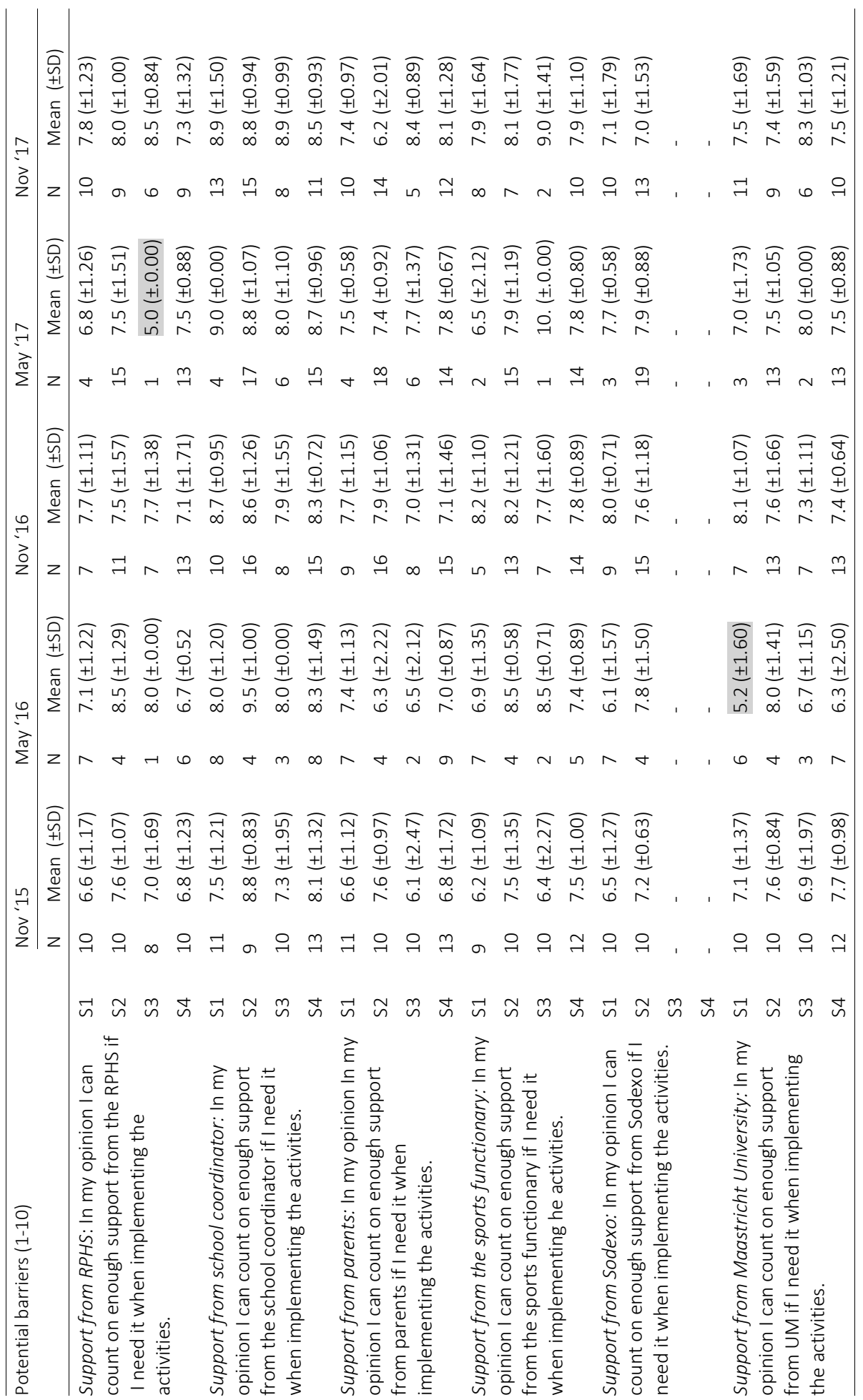




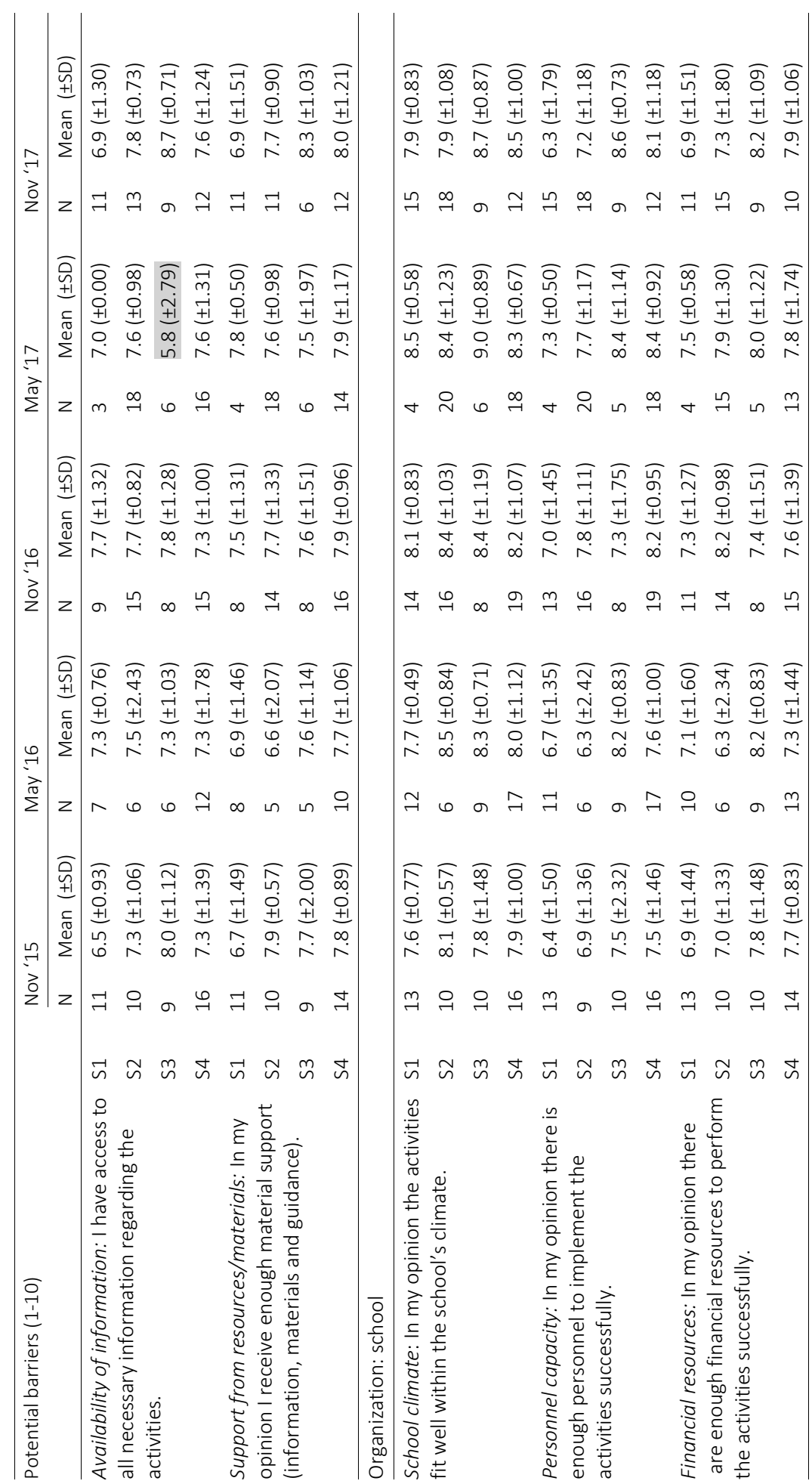




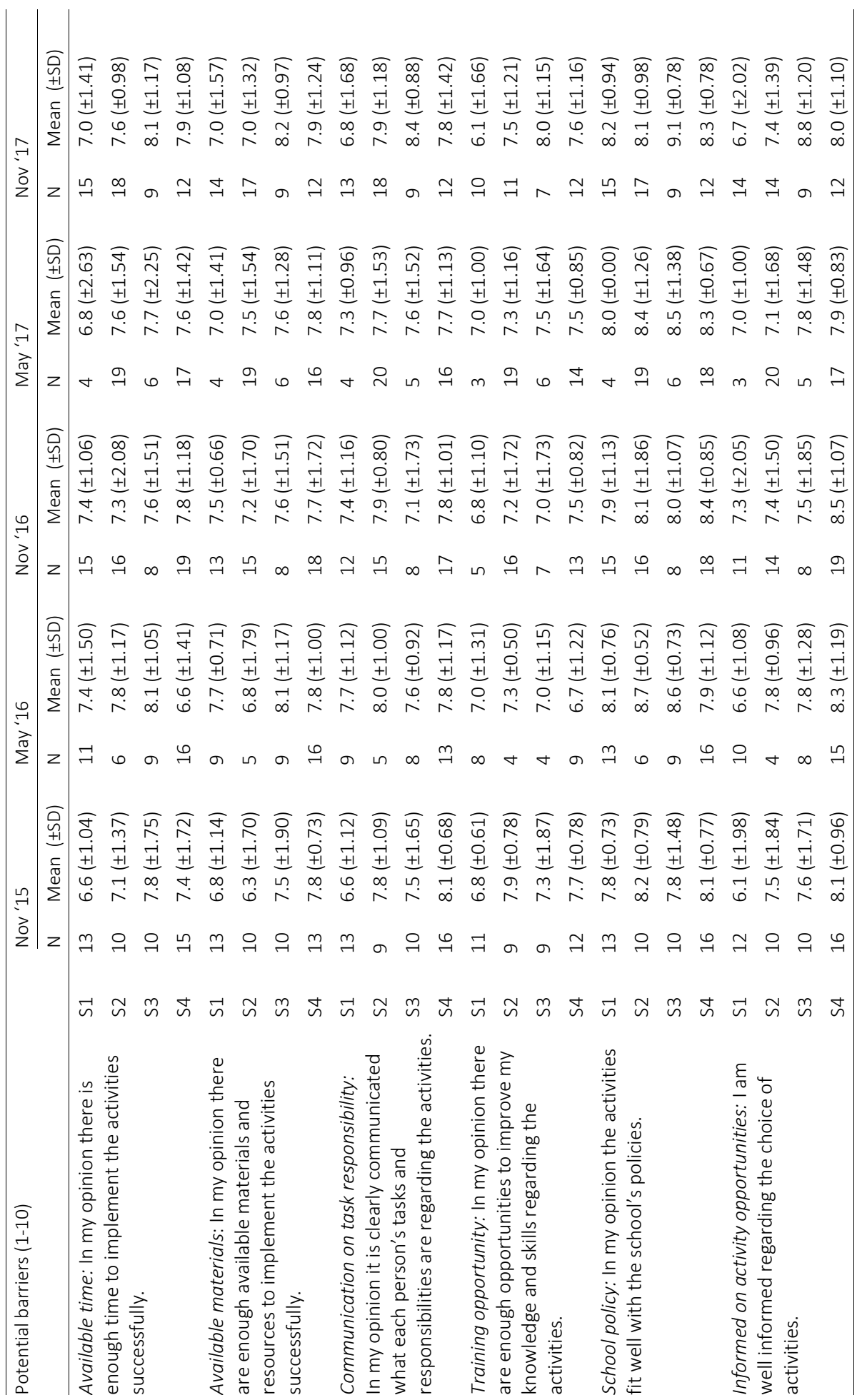




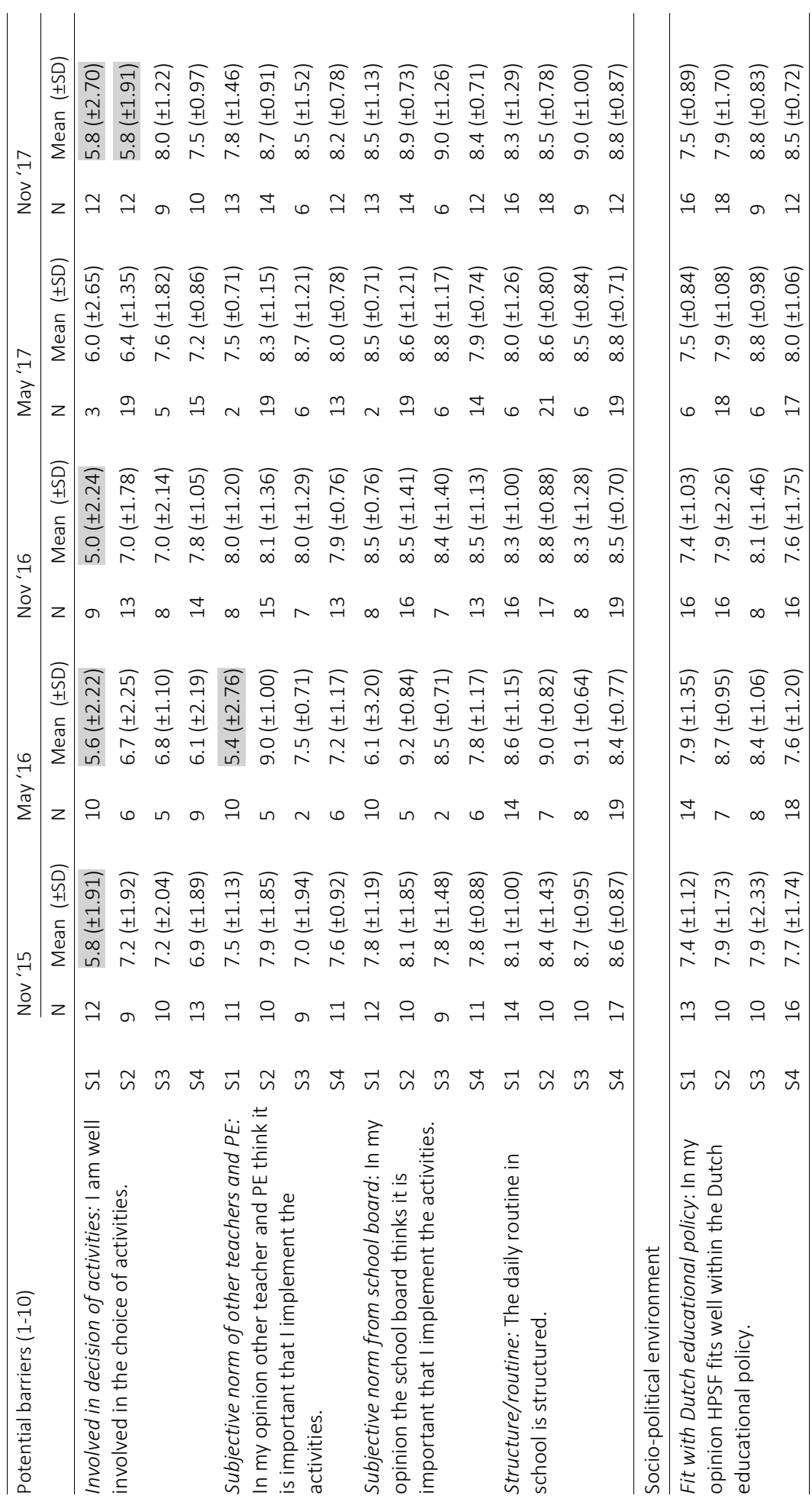




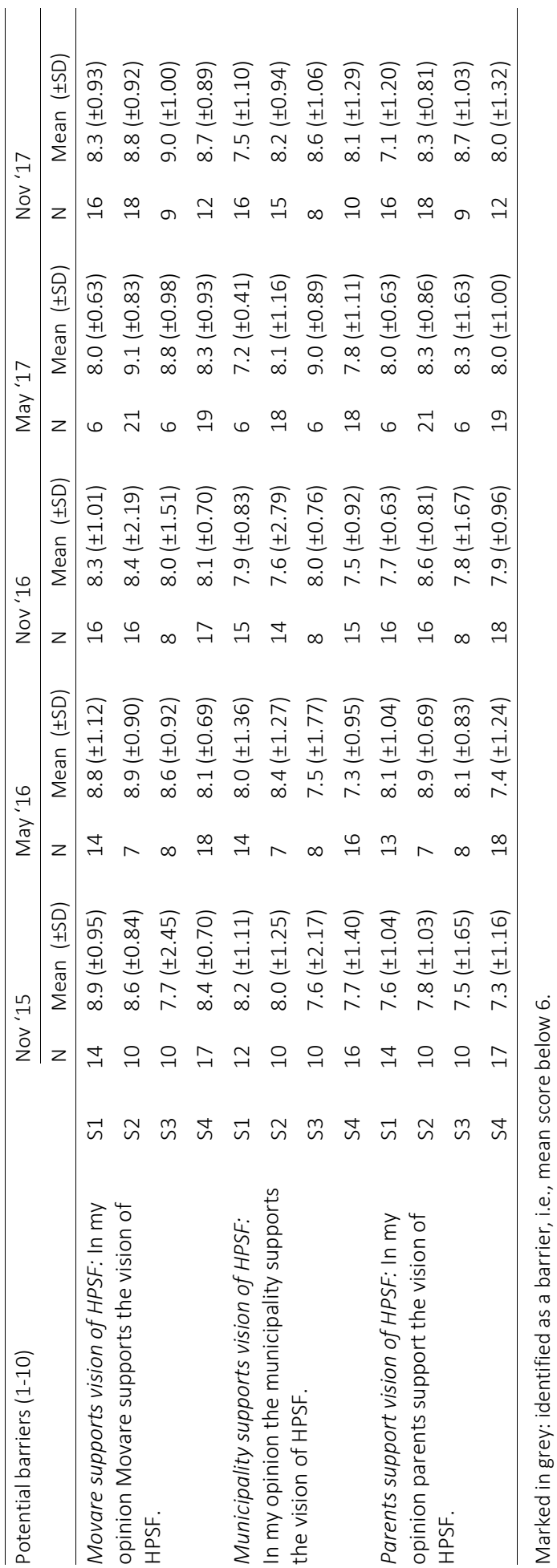




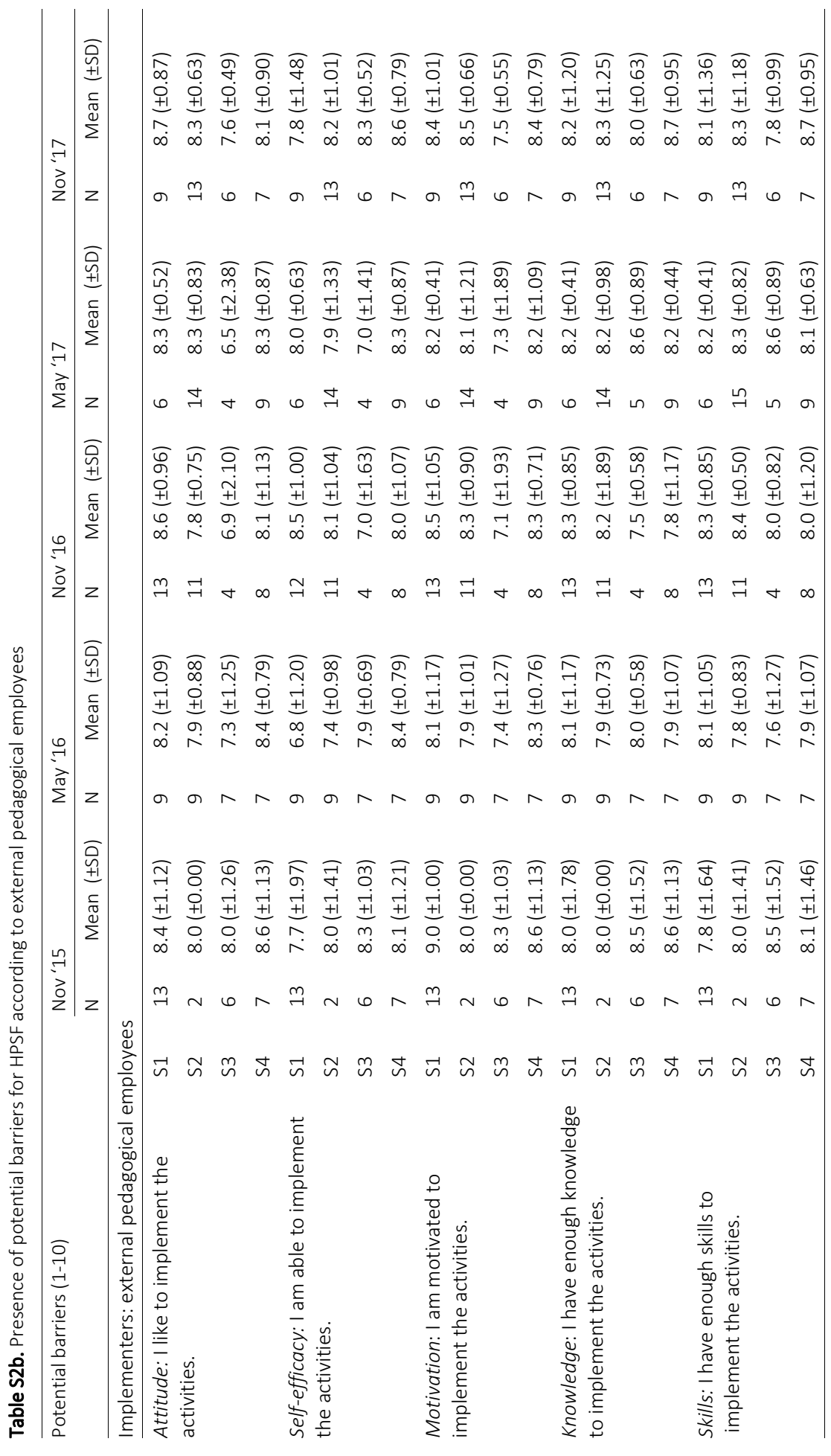




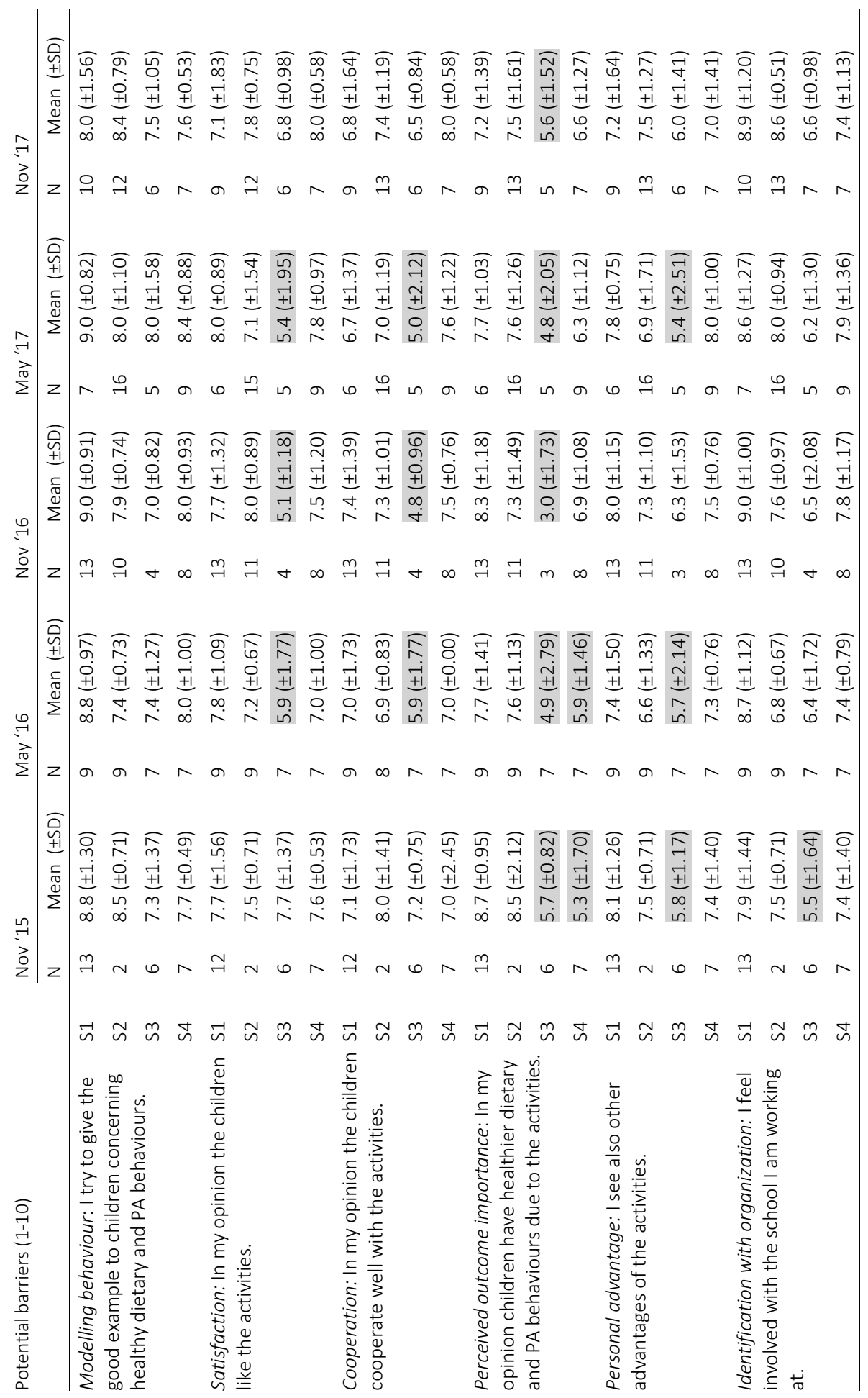




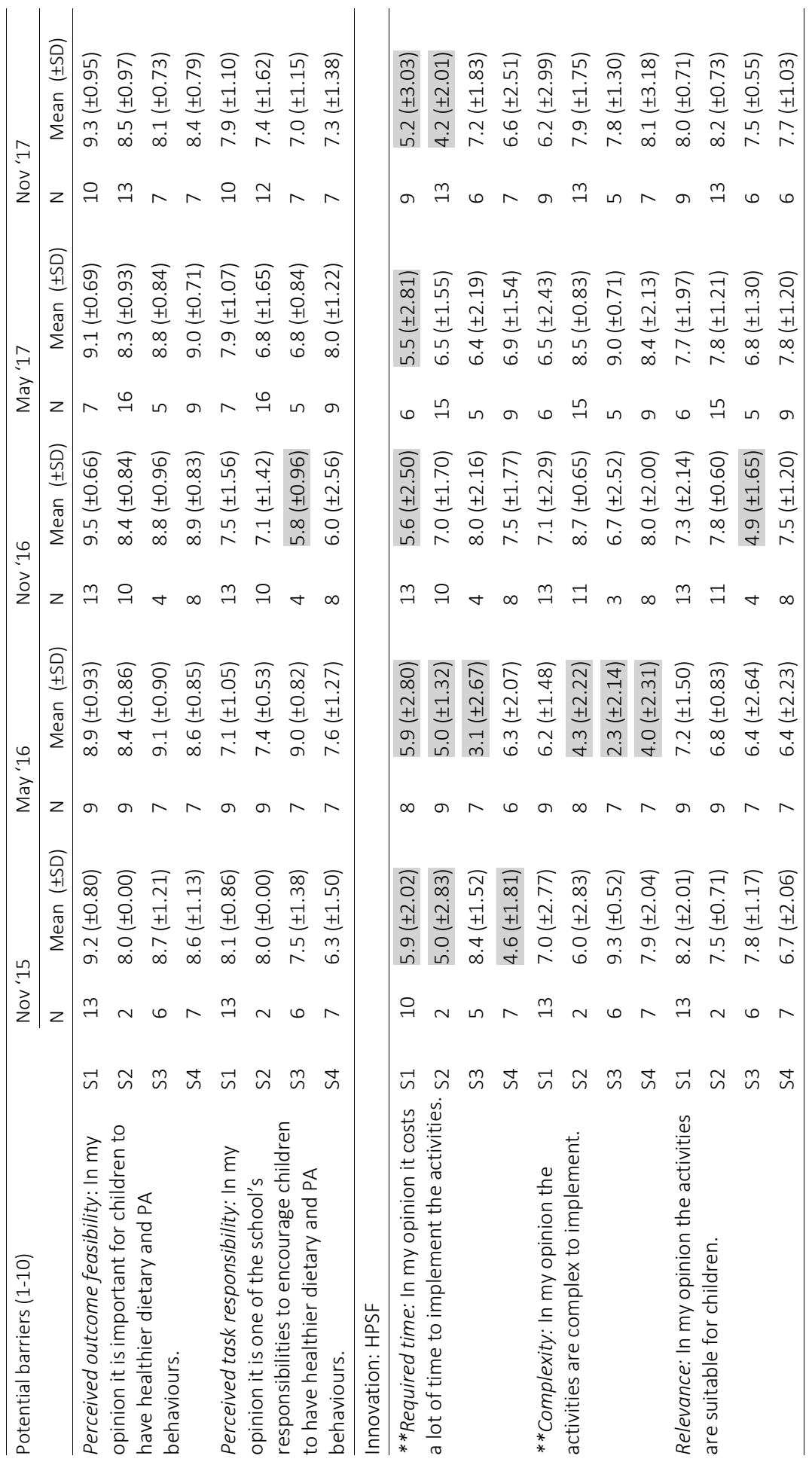




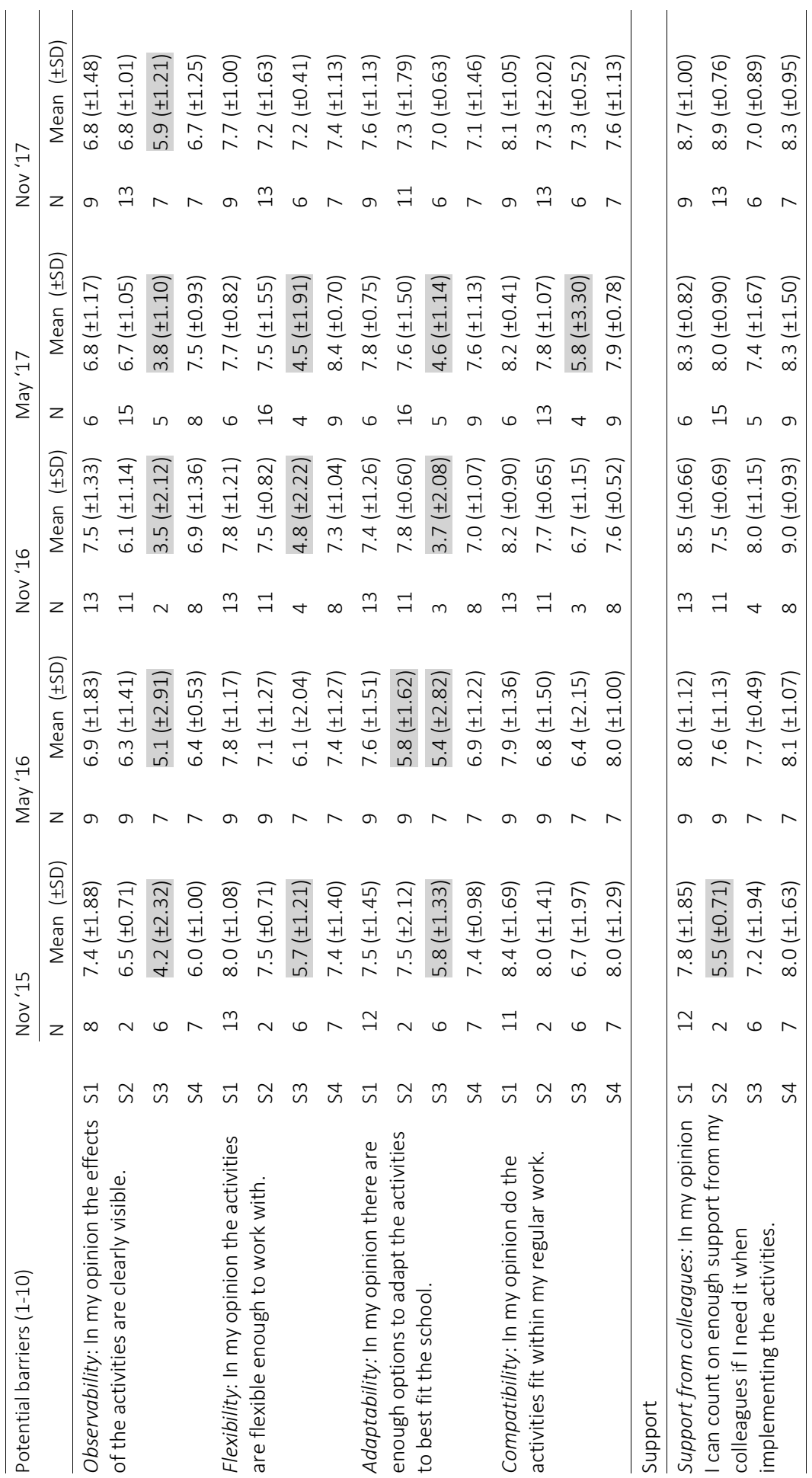




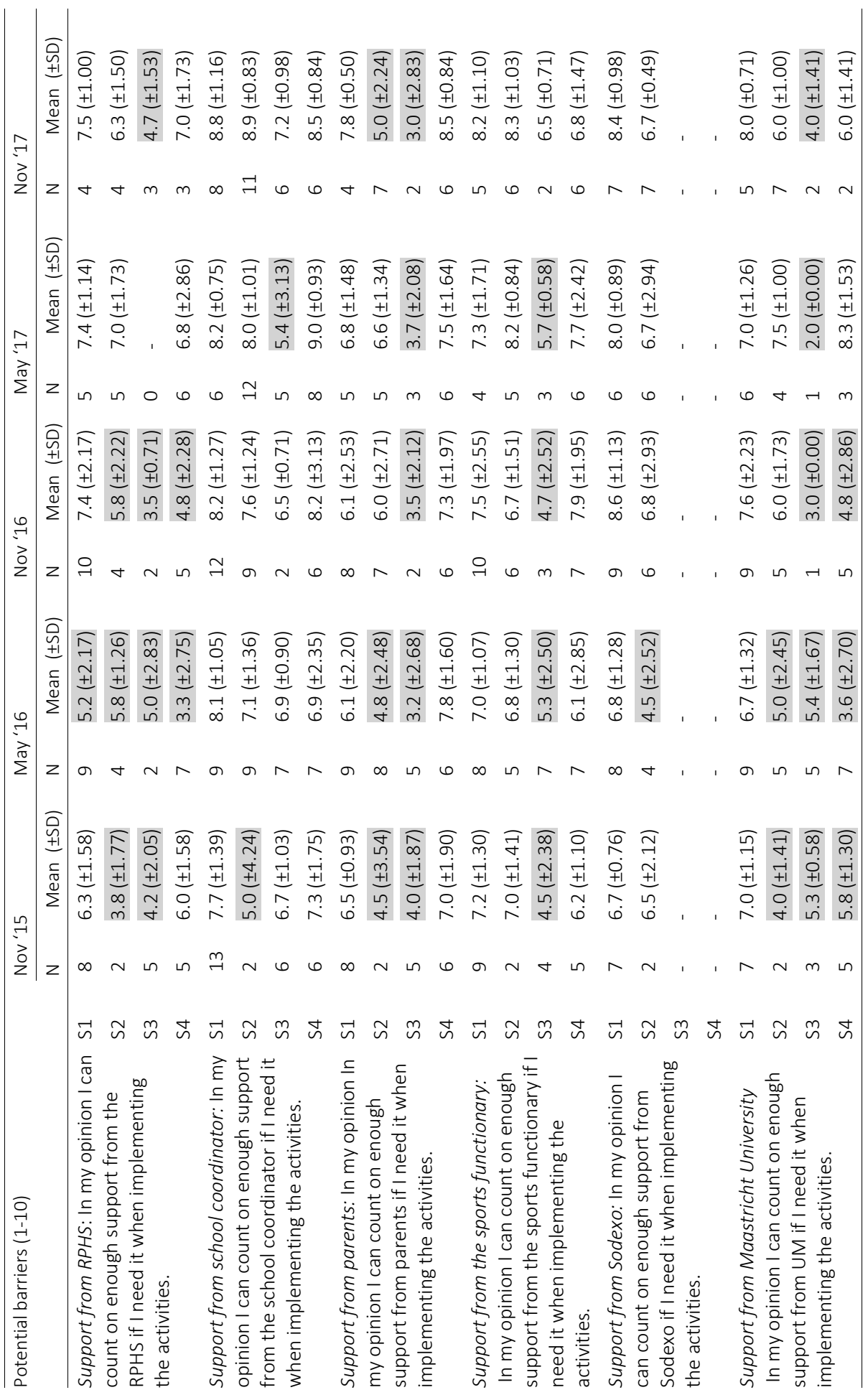




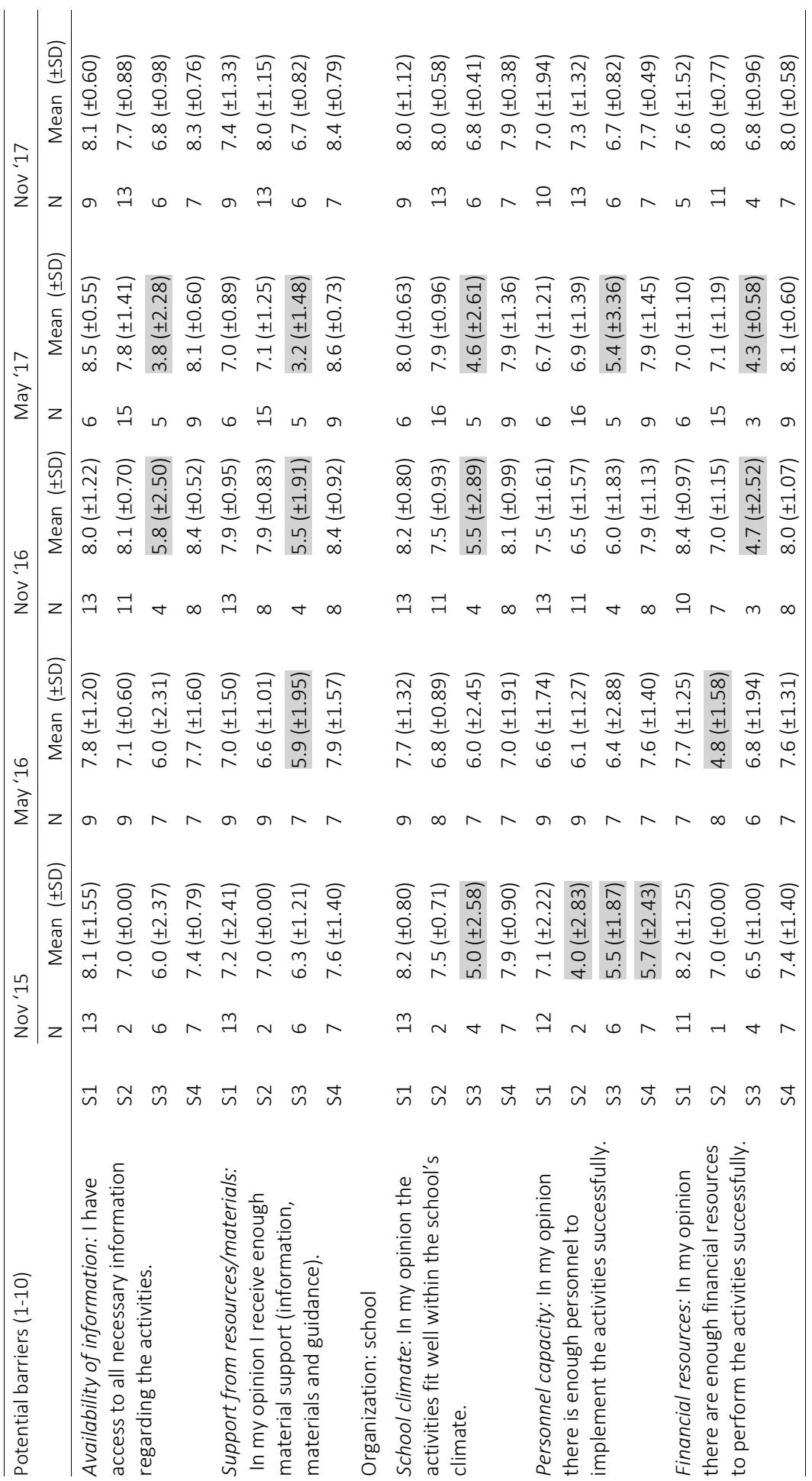




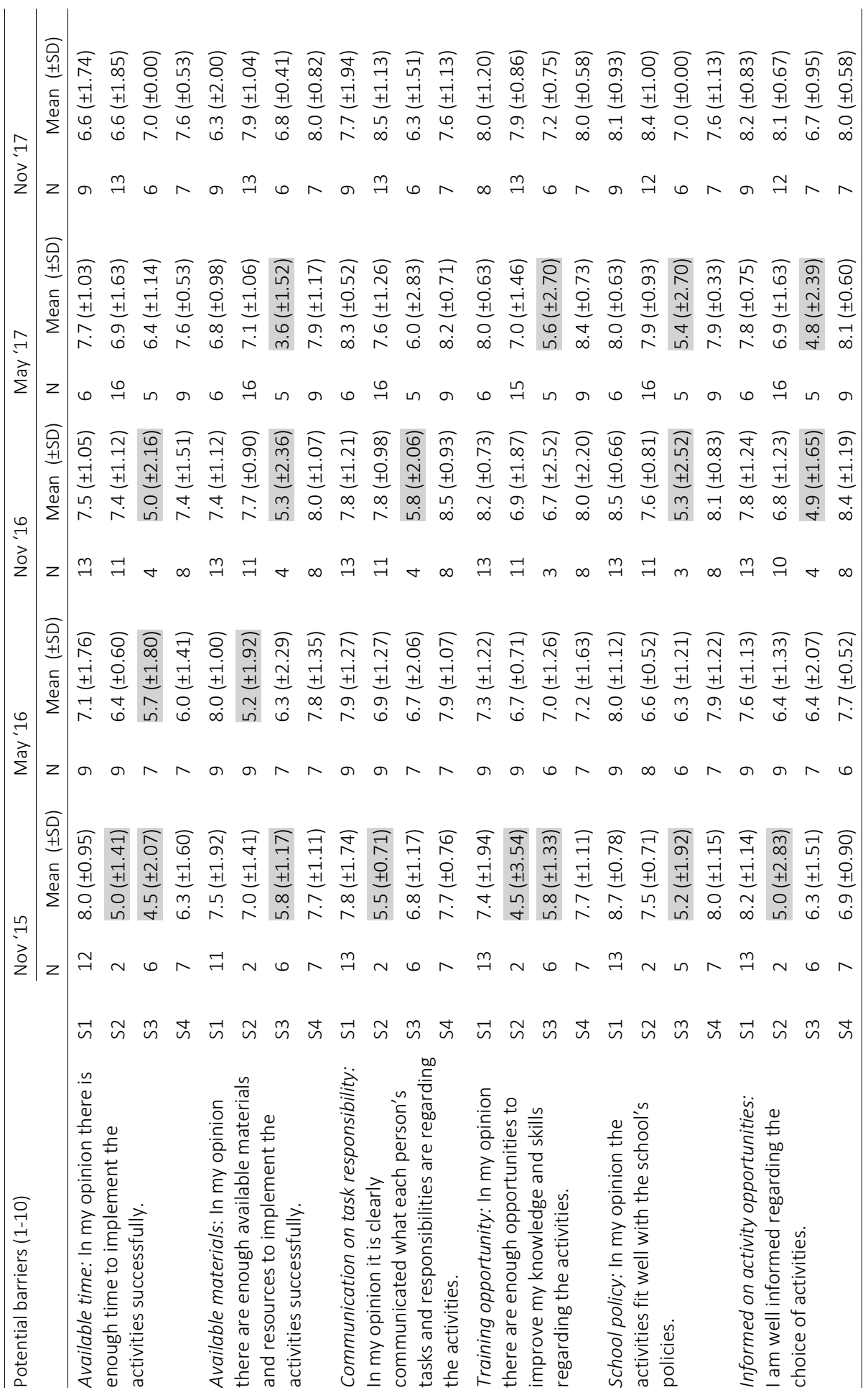




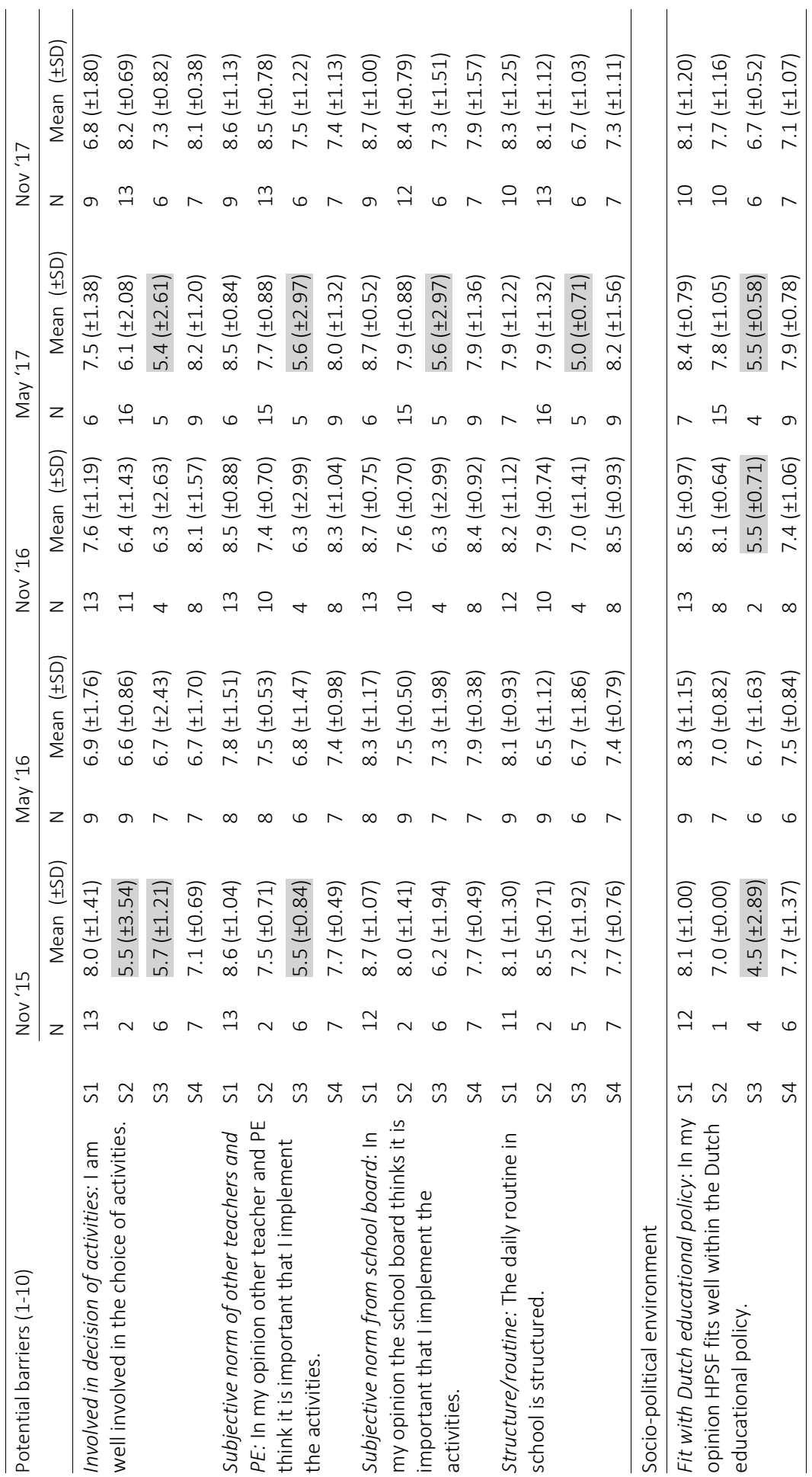




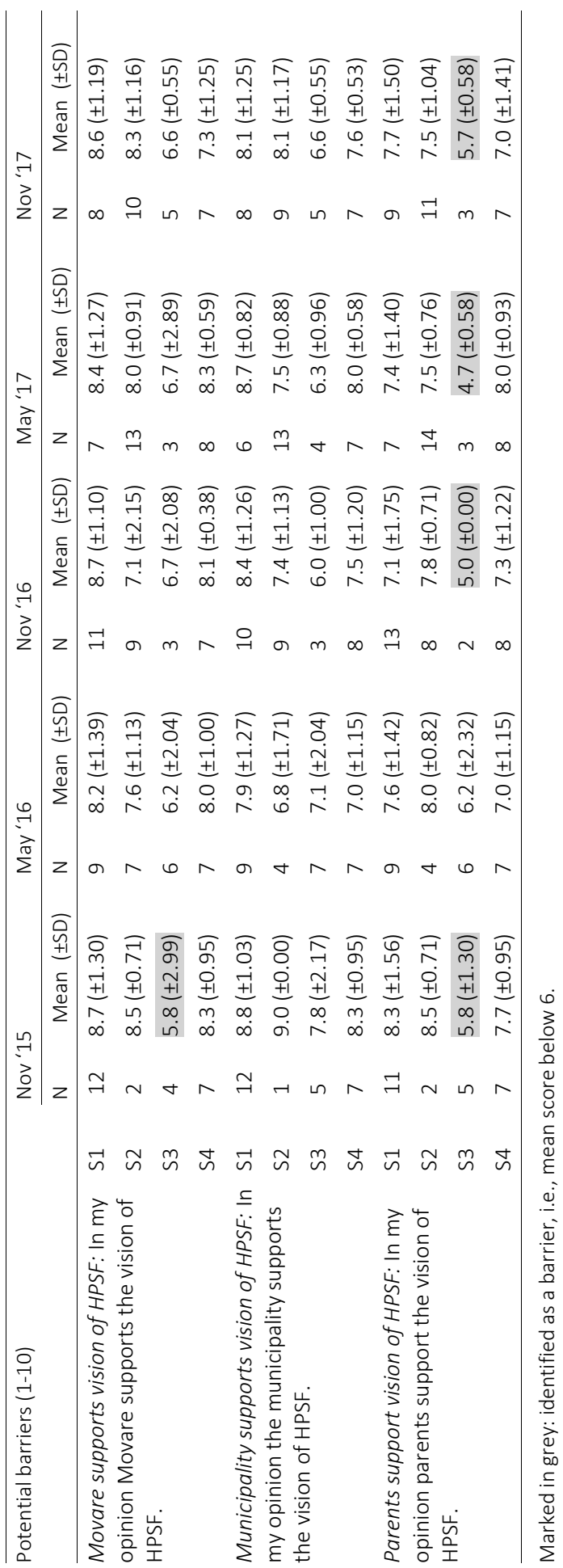




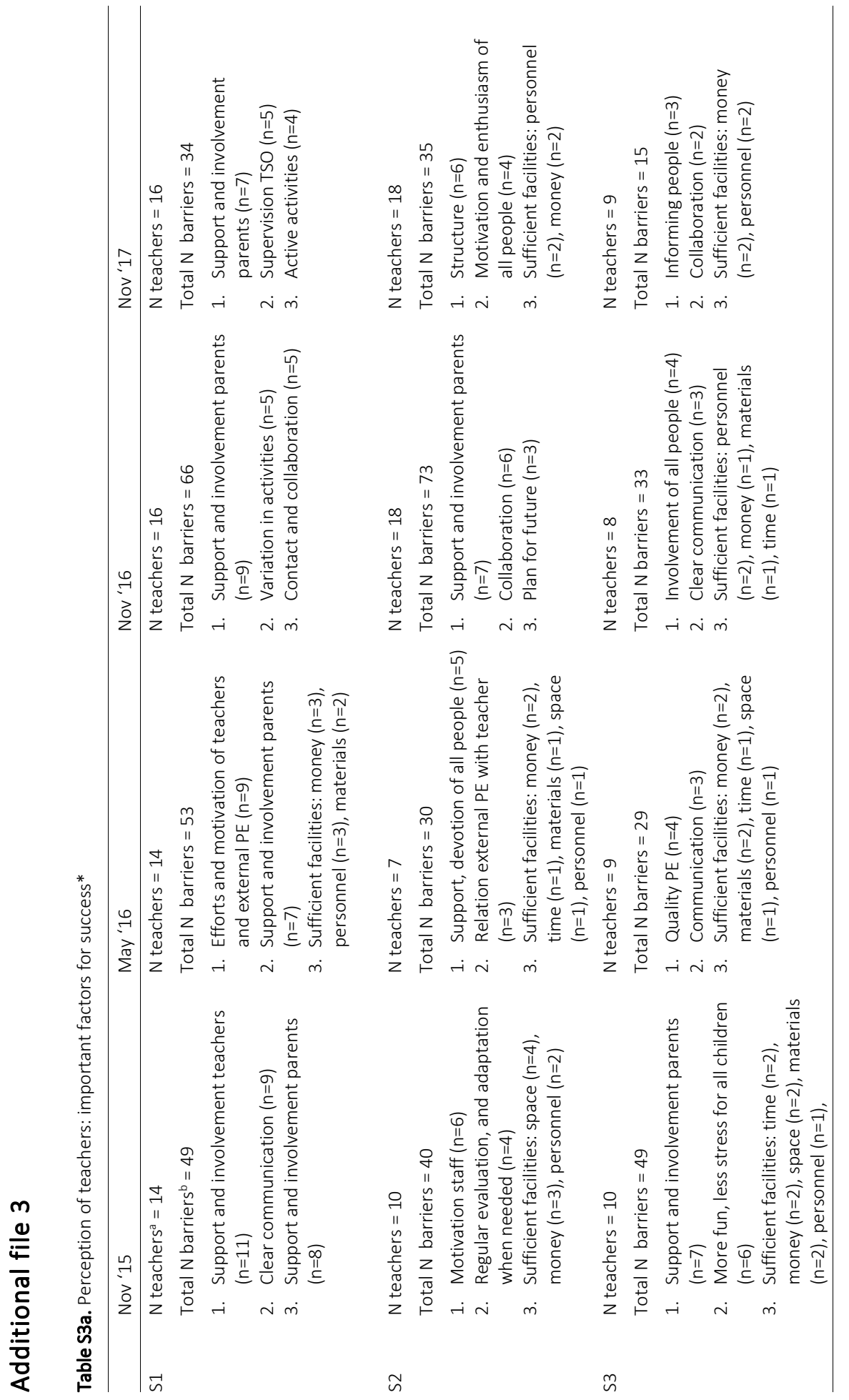




\section{Chapter 3}

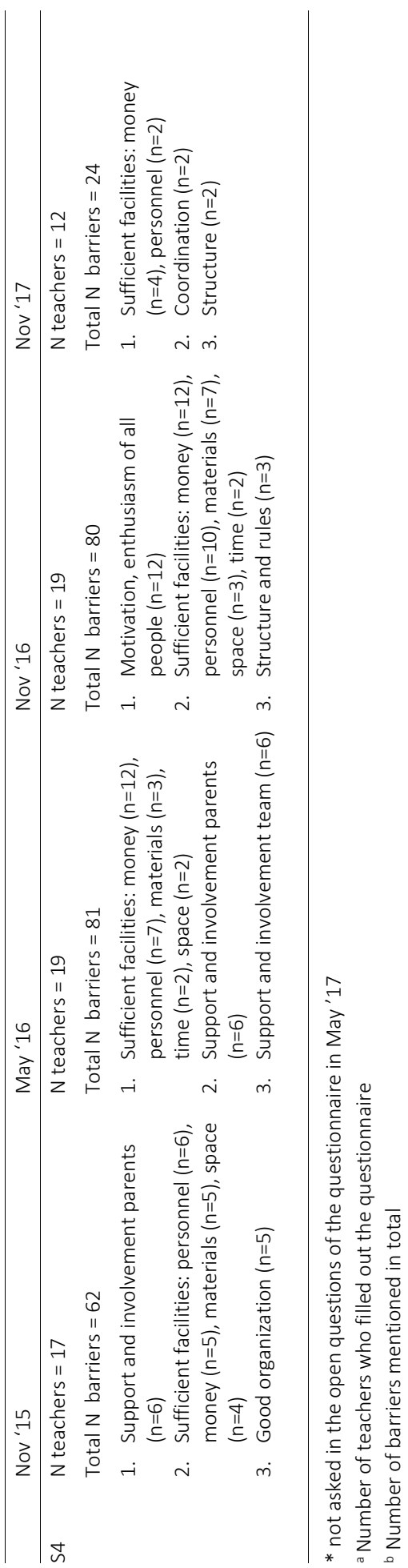




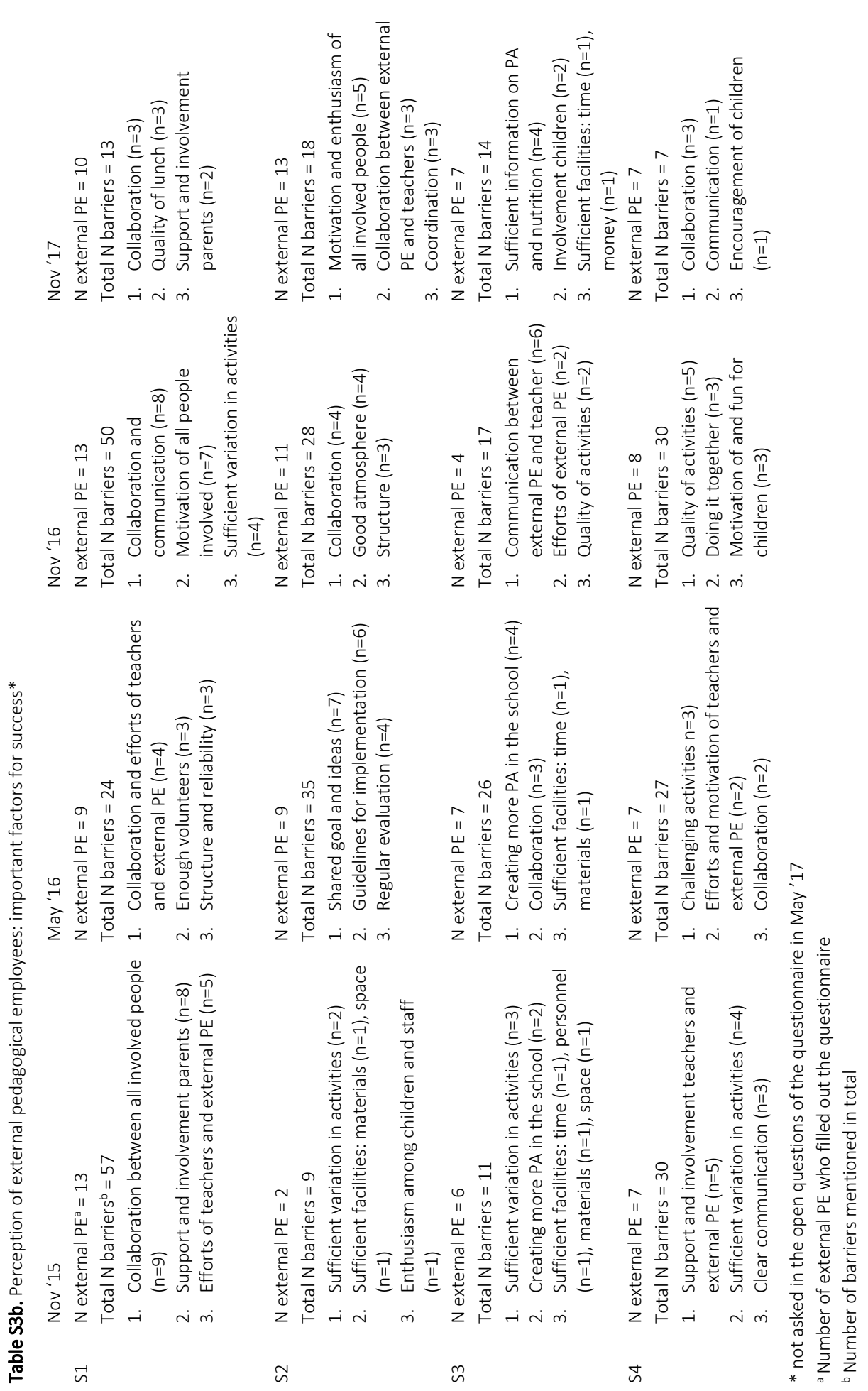





\section{Chapter 4}

Can the Healthy Primary School of the Future offer perspective in the on-going obesity epidemic in young children? - A Dutch quasi-experimental study

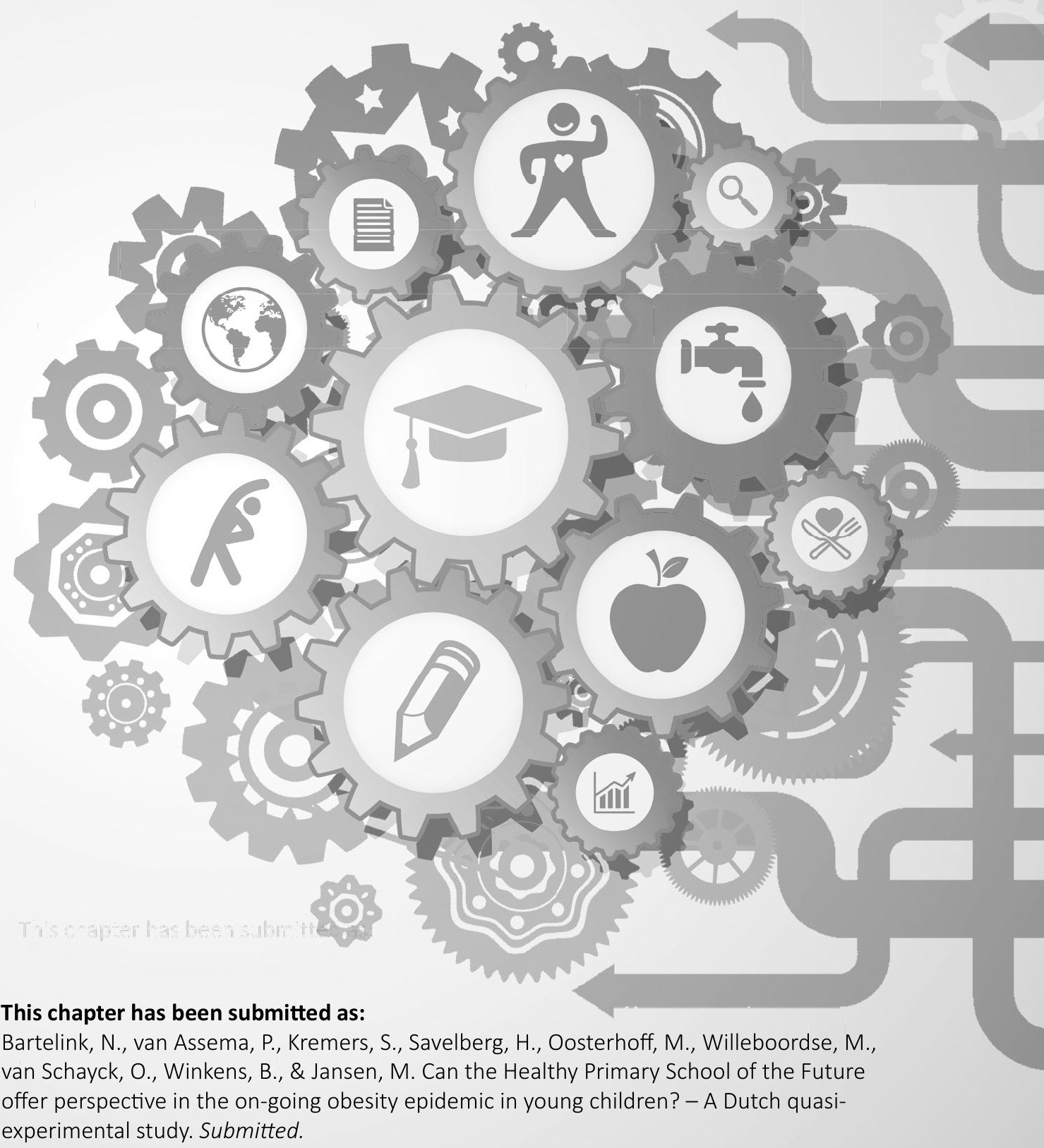




\section{Abstract}

\section{Objectives}

Schools play an important role in promoting healthy behaviours in children and can offer perspective in the on-going obesity epidemic. The 'Healthy Primary School of the Future' (HPSF) aims to improve children's health and well-being by enhancing school health promotion. The current study aims to assess the effect of HPSF on children's BMI z-score after one and two years' follow-up and to investigate whether HPSF has different effects within specific subgroups of children.

\section{Design}

A longitudinal quasi-experimental design.

\section{Setting}

Four intervention and four control schools participated; located in a low socioeconomic status region in the Netherlands.

\section{Participants}

1676 children (aged 4-12 years).

\section{Interventions}

HPSF uses a contextual systems approach and includes health-promoting changes in the school. Central to HPSF are the provision of a daily healthy lunch and structured physical activity sessions each day. Two intervention schools implemented both changes (full HPSF), two intervention schools implemented only the physical activity change (partial HPSF).

\section{Main outcome measures}

BMI z-score, determined by measurements of children's height and weight at baseline, after one and two years' follow-up.

\section{Results}

The intervention effect was significant after one-year follow-up in the partial HPSF (standardized effect size ES=-0.05), not significant in the full HPSF (ES=-0.04). After two years' follow-up, BMI z-score had significantly decreased in children of both the full HPSF (ES=-0.08) and the partial HPSF (ES=-0.07) compared with children of the control schools, whose mean BMI z-score increased from baseline to two years. None of the potential effect-modifiers (gender, baseline study year, socioeconomic status, and baseline weight status) were significant.

\section{Conclusions}

HPSF was effective after one and two years' follow-up in lowering children's BMI zscores. No specific subgroups of children could be identified who benefitted more from the intervention. 


\section{Introduction}

Overweight and obesity can lead to health problems, such as type 2 diabetes, cardiovascular diseases, and psychological problems (e.g., low self-esteem) [57, 105]. Globally, the prevalence of overweight and obesity among children and adolescents (aged 5-19) has risen dramatically from 4\% in 1975 to more than 18\% in 2016 [106, 107]. The prevalence is highest among children with a low socioeconomic background [10]. In the Netherlands, the prevalence of childhood overweight and obesity has also increased in the last decennia: $13-15 \%$ of children (aged 2-21 years) are overweight, and $1.8-2.2 \%$ are classified as obese, which is a 2- to 3 -fold increase compared with 1980 [5]. The on-going epidemic increase is particularly caused by unhealthy behaviours, such as unhealthy dietary intake and low levels of physical activity (PA) [56]. The health behaviours of children in the Netherlands are suboptimal. For example, $42 \%$ of children (aged 4-9 years) consume at least $150 \mathrm{~g}$ of fruit per day, which drops to $20 \%$ for 9-12 year olds [3]. Regarding PA, only half (48\%) of Dutch children (aged 4-12) meet the guidelines for PA of 60 min of moderate-to-vigorous physical activity (MVPA) per day [4]. Unhealthy behaviours at a young age often track into adulthood [6] and are related to health and psychosocial problems, reduced quality of life, higher health care costs, lower educational achievement, and labor participation [7-9]. A vicious circle is emerging, transferring problems, such as obesity, from one generation to the next [12]. Promoting healthy behaviours at an early age may help to improve children's health on the short and long run [6]. Moreover, promoting health behaviours could also lead to better educational and academic achievements, which have been found to be related to improved health in later life as well [58].

Schools can play an important role in promoting healthy behaviours in children since a significant proportion of a child's day is spent there and they reach all children [14, 59, 86]. As such, school-based interventions may be an important instrument to offer perspective in the on-going obesity epidemic in young children. Many different schoolbased interventions (e.g., related to education, environment, policy, and monitoring) have been implemented to integrate health into the school system and reduce childhood overweight and obesity. The meta-analysis of both Cook-Cottone et al. and Oosterhoff et al. found that the significant effect of school-based interventions on children's Body Mass Index (BMI) z-score had an overall weighted effect size of approximately $-0.05[108,109]$. Several studies indicated that effects are often hampered by underestimation of the challenges associated with implementing meaningful changes to the school system [20,32,42]. These challenges occur because an intervention always interacts with the specific school context [32, 42]. Therefore, solutions for the challenges associated with changing school systems vary between schools as they all have their own dynamics [32, 42, 47]. Consequently, an intervention can be seen as an attempt to positively disrupt the prior functioning of a school system 
$[54,90]$. Some other reviews stated that specific subgroups of children benefit more from a school-based intervention. The review of Stewart-Brown et al. [31] found that several studies indicated gender-specific results, with some school-based interventions being more effective in girls and others in boys. Age-specific effects were often found, with some interventions being more effective in older children and others in younger children [31]. Cook-Cotton et al. found that children's socioeconomic background can be an influential factor and that children already having overweight can respond more slowly or to a lesser extent to school-based interventions than children with a healthy weight [108].

A Dutch initiative that embraces a contextual systems approach is the 'Healthy Primary School of the Future' (HPSF) [55, 92]. HPSF aims to improve the health and well-being of all children in the school which should contribute to a healthier future generation and thereby offer perspective in the on-going obesity epidemic [91]. HPSF includes topdown and bottom-up processes to create health-promoting changes in the school. Two changes were initiated to create some form of positive disruption in the school: 1) providing a free healthy lunch each day and 2) daily structured physical activity (PA) sessions after lunch. While in other national school systems this may represent usual practice, these changes are hypothesized as disruptive to the Dutch school system because the provision of school lunches and structured PA sessions are not usual practice in Dutch schools. The two changes aimed to create momentum to implement additional health-promoting changes in the school, such as a healthy school policy or creating a PA-friendly schoolyard. All changes together should favourably affect the health behaviours of all school children, which should lead to improved health and a more normal weight status $[55,92]$.

The aim of the current study was to assess the effect of HPSF on children's BMI z-score after one and two years' follow-up and to investigate whether HPSF has different effects within specific subgroups of children. The current study is part of an overall study to investigate HPSF. The overall study has a broad scope and includes a multidisciplinary research group, which focuses on many different outcomes, such as children's health behaviours, educational achievements, and well-being. The studies that have been published previously, focused on the implementation process of HPSF [110] and the effects of HPSF on children's dietary and PA behaviours [111]. The current study explicitly concentrates on children's BMI z-score to focus in much detail on the primary outcome as described in the study design of Willeboordse et al. [55]. 


\section{Methods}

\section{Study design}

The current study had a longitudinal quasi-experimental design with four intervention schools and four control schools, which maintained the school curriculum that is currently common practice in the Netherlands. Ethical approval (14-N-142) was given by the Zuyderland Medical Ethics Committee located in Heerlen (Parkstad, the Netherlands). Parents had to sign an informed consent form to participate in all measurements for themselves and their child(ren). Measurements were conducted in September-November of 2015 (T0), 2016 (T1) and 2017 (T2). A detailed description of the study and the power calculation is reported in Willeboordse et al. [55].

\section{The Healthy Primary School of the Future}

Three collaborating organizations, i.e., the regional educational board 'Movare', the regional public health services and Maastricht University, developed the idea for HPSF [55]. In March 2013, 12 out of 53 schools governed by the Movare educational board were informed about the initiative. Four schools gave their initial consent and spent a whole school year (2014/2015) creating bottom-up support for HPSF. Two of the four intervention schools decided to implement both the daily lunch and the structured PA sessions and are referred to as the 'full HPSF'. The other two intervention schools decided to only implement the structured PA sessions, and are referred to as the 'partial HPSF'. All schools could implement additional health-promoting changes, that fit their school context $[92,110]$. The full HPSF improved their health policy, provided water bottles to all children, and provided an educational lunch once a week. The partial HPSF did not implement additional health-promoting changes.

Implementation started in all four intervention schools in November 2015. The time for having lunch (in the full HPSF) was increased to 20-30 min. The total lunch break time in these schools was prolonged by about $60 \mathrm{~min}$. For this reason, the school day was extended: children of the full HPSF attend school to approximately 15:30/15:45 instead of 15:00. A dietician of the caterer developed a lunch menu cycle that changed every 10 weeks, in which at least $80 \%$ of the products met the advice of the Dutch Health Council [112]. A mid-morning snack, consisting of fruits and/or nuts, was also provided. The lunch, a bread-based cold meal, was typically Dutch. During lunch break time, the children participated several times a week in structured PA sessions; one or two times per week they could participate in cultural activities. The PA sessions were carried out in the schoolyard and when available and needed, in parks, forest, and/or sports hall in the neighbourhood. All schools collaborated with sport clubs or other external partners to offer specific activities as well. Since the two changes were 
contextualized bottom-up, this resulted in some differences between schools in the form of the changes; the content remained comparable.

The two changes, i.e., providing daily a free healthy lunch and structured PA sessions after lunch, were both led by external pedagogical employees provided by childcare organizations to not increase the workload of teachers even further. This integration of the childcare organization during school hours is not to provide a temporary solution, but to change the school's organization in a sustainable way. The aim for the future is to bring school and childcare more together and thereby create an integrated day for children, whereby children are supervised by the same people prior, during and after school hours. Employees of sports and leisure organizations supported the external pedagogical employees during implementation when needed, and after a year they provided a training course ( 8 sessions of $2 \mathrm{~h}$ ) to supply them with additional tools for how to motivate children for active participation during the PA sessions. A health promoter from the regional Public Health Services was assigned to each school to provide support when needed. In this study, researchers from Maastricht University monitored and fed back results to the schools to support the processes of change. Funding for implementation of HPSF is provided by the provincial authorities until the end of 2019. However, the four schools have committed to continued implementation after 2019 and make the changes sustainable in their school.

\section{Patient and public involvement}

Public involvement was a key feature of HPSF. This intervention intended to establish a co-creation movement in schools aimed at the systematic incorporation of health and well-being. The two top-down changes and the additional health-promoting changes were developed and contextualized by bottom-up involvement. Teachers and parents were involved from the start in the adoption decision and the process of adapting the several changes into the school context. Moreover, all four schools used a children voice group, with representatives from each class in school, to get insight into the opinion of children regarding HPSF. In this way, the experiences of children were being heard and the changes could be further contextualized to fit better to the children's needs and wishes. Each of the four intervention schools selected a teacher as school coordinator, who managed HPSF in their school. Overarching, HPSF was led by an executive board with representatives of the three collaborating organisations: Movare, the regional Public Health Services and Maastricht University. They discussed the study design, the relevant outcome measures, and the interpretation of the results. The representative of Movare advised explicitly on school and participant recruitment and the communication to schools. A project team was created with representatives of all partners involved: the four schools, Movare, regional Public Health Services, Maastricht 
University, the Limburg provincial authorities, childcare organizations, the caterer, and sports and leisure organizations. No patients were involved in this study.

\section{Study population}

All intervention and control schools are situated in the Parkstad region in the southern part of the Netherlands. This region has a low average socioeconomic status (SES), and unhealthy lifestyle behaviours and overweight are highly prevalent compared with the rest of the Netherlands [40]. More information on the recruitment of the schools has been described elsewhere [55]. All children ( $N=2326$ at T0) and their parents in the eight schools were invited to participate in the study. This included children from study year one to eight (age 4 to 12 years), which is comparable to two years of Kindergarten and six primary school grades. Recruitment was done via information brochures for parents. In addition, the research team visited the classrooms to inform children about the study and encourage them to ask their parents for participation [55]. Due to the dynamic population in the schools (new children enter and other children finish school each year), we focused in this study only on the children who were enrolled in the schools at baseline till the end of this 2-year study. The population of children included in this study were: at baseline (TO) children from study year one to seven, at T1 children from study year two to eight, and at T2 children from study year three to eight. Children of these study years who joined the study at T1 or T2 were included, even though no baseline data was available. Even though these children joined the study later, they were at baseline already participating in their school and thus also exposed to HPSF during the full 2 years of this study. Children who switched to other schools between 2015 and 2017 were excluded.

\section{Measurements}

In each school, the data were gathered annually during one week of measurements. Inter-rater variability was minimised by training researchers according to a strict protocol [55]. Children's age, study year, and gender were collected via the database of the educational board Movare. A digital questionnaire for parents was used to obtain information about the children's socioeconomic background and ethnicity. SES was calculated as the mean of standardized scores on maternal education level, paternal educational level, and household income (adjusted for household size) [113]. The mean scores were categorized into low, middle and high SES scores based on tertiles. Children's ethnicity was determined by the country of birth of both parents and divided into 1) Western background (including the Netherlands) and 2) non-Western background [114]. If one of the parents was born in a non-Western country, the child's ethnicity was assigned to non-Western. The distinction between Western and nonWestern was created because of differences in socioeconomic and cultural position between the two backgrounds [114]. 


\section{$\underline{B M I}$ z-score}

Anthropometric measurements, i.e., height, weight, hip and waist circumference, were conducted in children from study year two to eight. The measurements were integrated in the school hours allocated to physical education. Weight was measured to the nearest $0.1 \mathrm{~kg}$ (Weighing Scale 803, Seca, Hamburg, Germany) and height was measured to the nearest $0.1 \mathrm{~cm}$ (Stadiometer 213, Seca, Birmingham, United Kingdom). Hip and waist circumference were measured with a measuring tape to the nearest 0.1 cm (model 201, Seca, Hamburg, Germany). Children were measured with light sports clothing and no shoes. All anthropometric measurements were performed twice, and a third measurement was conducted if the difference between the first two measurements exceeded a pre-set limit (weight $\geq 0.2 \mathrm{~kg}$, height $\geq 0.5 \mathrm{~cm}$, hip and waist circumference $\geq 1.0 \mathrm{~cm}$ ). Unfortunately, hip and waist circumference were excluded from further analyses due to measurement errors. BMI was assessed by height and weight; age- and gender-specific BMI cut-off points were used to define overweight and obesity [115]. BMI z-scores were calculated by using Dutch reference values [5].

\section{Statistical analyses}

Data were analyzed using IBM SPSS Statistics for Windows (version 23.0. Armonk, NY: IBM Corp). Pearson's chi-square tests and ANOVA tests were conducted to analyze the comparability of the observed participant characteristics among the full HPSF, the partial HPSF, and control schools at baseline. Linear mixed model analyses were used to assess the longitudinal intervention effects on the children's BMI z-score. Since measurements were repeated within participants, we used a two-level model with repeated measurements as the first level and participants as the second level, where an unstructured covariance structure was considered for the repeated measures. The fixed part of the model consisted of group (full HPSF, partial HPSF, and control), time (T0, T1, T2) and the interaction terms of group with time. We were not able to include class as a level in the model, because often several divisions of one class existed, e.g., 4 a or $4 \mathrm{~b}$, and children often did not have fixed class divisions for all years. All analyses were adjusted for gender, study year at baseline, SES, and ethnicity. Missing covariates and BMI z-scores were imputed using multiple imputation method with fully conditional specification (FCS) and 10 iterations, generating 50 complete datasets. Gender, study year at baseline, school type, ethnicity, SES score, and BMI z-score were used to impute the missing data. We performed two sensitivity analyses. First, we replicated the analyses by only selecting the children who had no missing BMI z-score at all three time points (complete-case analysis). Second, we replicated the analyses while excluding children with an extremely low BMI z-score at baseline (BMI z-score $\leq-2$ ), to study the effects only in children for which a decrease in BMI z-score is favourable. To study whether the intervention effects were similar for all subgroups of children, the 
following potential effect modifiers were considered: gender (boys/girls), study year at baseline (lower (1-4)/higher (5-8) grades), SES (low/middle/high), and baseline weight status (non-overweight/overweight). To assess this potential effect modification, the interaction term group*time*effect modifier, with all corresponding two-way interactions, was added to the above mentioned model. If this interaction term was significant (here we used a significance level of 0.10 to deal with the fact that the power of a test for interaction is relatively low and we did not want to miss any effectmodification), the intervention effects were reported for all categories of the effectmodifier separately. For all other analyses, a two-sided p-value $\leq 0.05$ was considered statistically significant. Standardized effect sizes (ES) at each time-point were included, which were defined as estimated mean difference at that time point (T1 or T2) divided by the square root of the residual variance at baseline (pooled over all three groups).

\section{Results}

Of all children ( $n=2326$ ) invited to participate in the (overall) study, 60.3\% joined the study at baseline ( $n=1403$ ) (Figure 1). Because of the study's dynamic population, a total of 1974 children and their parents participated in the study within the two-year followup period (data collected at one time-point at least). Due to the selection used for the current study, i.e., only including the children who were in study year one to seven at baseline, we included 1676 children in the analysis. Of these children, $47.4 \%$ were boys, their mean age was 7.5 years old, and $94.1 \%$ had a Western ethnicity (Table 1 ). In total, $19.9 \%$ of these children suffered from overweight or obesity, which is higher compared with the national average of $13 \%$ [116]. BMI z-scores at baseline differed significantly between the three school groups $(p=0.034)$ : the average BMI $z$-score of children in the control schools ( $z$-score $=0.232$ ) was higher compared with children in the full HPSF ( $z$ score $=0.051)$ and the partial HPSF (z-score=0.092). Significantly more children suffered from overweight or obesity in these control schools $(24.1 \%)$ than the full HPSF $(16.5 \%)$ and the partial HPSF (17.9\%) $(p=0.006)$. 


\begin{tabular}{|c|c|c|}
\hline \multicolumn{3}{|c|}{$\begin{array}{l}\text { Baseline (T0) } \\
\text { ildren: } n=1403 \text { ( } 60.3 \% \text { of all children) }\end{array}$} \\
\hline \multicolumn{3}{|c|}{$\begin{array}{l}\text { Selection for effect study } \\
\text { Participating children in classes 1-7: } n=1255\end{array}$} \\
\hline Full HPSF (n=361) & Partial HPSF $(n=408)$ & Control $(n=486)$ \\
\hline \multicolumn{3}{|c|}{$\begin{array}{l}\text { One-year follow-up (T1) } \\
\text { Total participating children: } n=1489 \text { (60.7\% of all children) }\end{array}$} \\
\hline \multicolumn{3}{|c|}{$\begin{array}{l}\text { Selection for effect study } \\
\text { Participating children in classes } 2-8: n=1455 \\
\text { Newly included }(n=264) ; \text { Drop-out* }(n=64)\end{array}$} \\
\hline $\begin{array}{l}\text { Full HPSF ( } n=469) \\
\text { New included: } n=132 \\
\text { Drop-out: } n=24\end{array}$ & $\begin{array}{l}\text { Partial HPSF }(n=428) \\
\text { New included: } n=33 \\
\text { Drop-out: } n=13\end{array}$ & $\begin{array}{c}\text { Control }(\mathbf{n}=\mathbf{5 5 8}) \\
\text { New included: } n=99 \\
\text { Drop-out: } n=27\end{array}$ \\
\hline \multicolumn{3}{|c|}{$\begin{array}{l}\text { Two-year follow-up (T2) } \\
\text { Total participating children: } n=1470 \text { (61.7\% of all children) }\end{array}$} \\
\hline \multicolumn{3}{|c|}{$\begin{array}{l}\text { Selection for effect study } \\
\text { Participating children in classes 3-8: } n=1323 \\
\text { Newly included }(n=158) ; \text { Drop-out** }(n=290)\end{array}$} \\
\hline 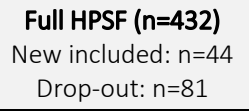 & $\begin{array}{l}\text { Partial HPSF }(n=376) \\
\text { New included: } n=38 \\
\text { Drop-out: } n=90\end{array}$ & $\begin{array}{c}\text { Control }(\mathbf{n}=\mathbf{5 1 5}) \\
\text { New included: } n=76 \\
\text { Drop-out: } n=119\end{array}$ \\
\hline \multicolumn{3}{|c|}{ Total participating children in study period TO-T1-T2: $n=1974$} \\
\hline \multicolumn{3}{|c|}{ Total selection for effect study***: $n=1676$} \\
\hline Full HPSF ( $n=537)$ & Partial HPSF ( $n=478$ ) & Control ( $n=661)$ \\
\hline
\end{tabular}

Figure 1. Flowchart

* Reasons for drop-out T1: switched to other included school $(n=2)$, other reasons, e.g., moved away or actively stopped participation $(n=62)$.

** Reasons for drop-out T2: finished school ( $n=228)$, switched to other included school $(n=17)$, other reasons e.g. moved away or actively stopped participation $(n=45)$.

***Selection for effect study: at baseline (TO) children from study year one to seven, at T1 children from study year two to eight, and at $\mathrm{T} 2$ children from study year three to eight. 
Effect of HPSF on children's BMI z-score

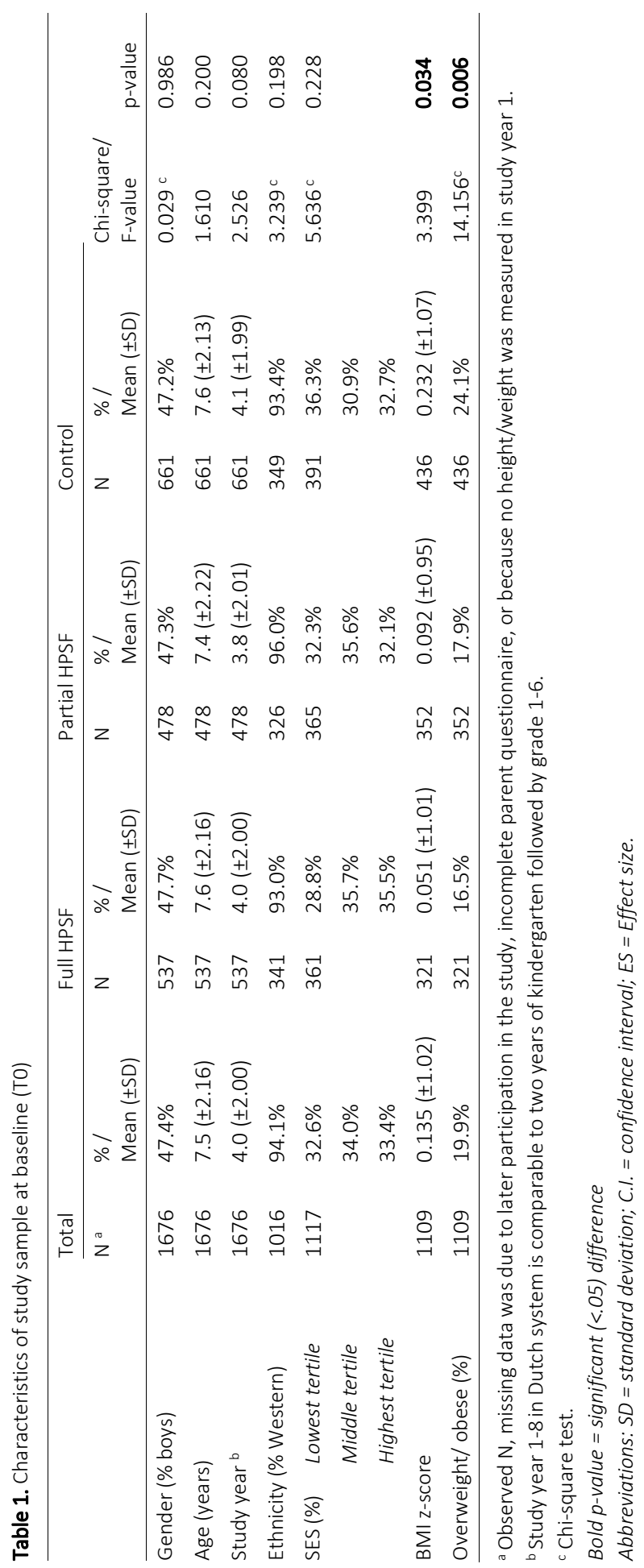


Observed data at T1 showed a decrease in BMI z-score compared with baseline in all three groups, with the full HPSF ( $\Delta z$-score: -0.074$)$ and the partial HPSF ( $\Delta z$-score: 0.098 ) having the largest decrease, and control schools a smaller decrease ( $\Delta z$-score: 0.018). At T2, a decrease in BMI z-score compared with baseline was observed in the full HPSF ( $\Delta z$-score: -0.039$)$ and the partial HPSF ( $\Delta z$-score: -0.012$)$, and an increase in the control schools ( $\Delta z$-score: +0.058 ) (Figure 2 ). The extent of observed increase or decrease at T1 and T2 compared with baseline of individual children in the three different groups is visualized in Figure S1 (Additional file 1). This figure shows that compared to the control schools, in the full and partial HPSF a higher percentage of children had decreased BMI z-scores. This was particularly visible after one-year followup. The figure also indicates that, on an individual level, mostly minimal to moderate changes $(-0.6 \leq \Delta z$-score $\leq+0.6)$ were realized. The variation in changes increased over time, i.e., the percentage of large and extreme decreases and increases was larger after two years' follow-up compared with one-year follow-up.

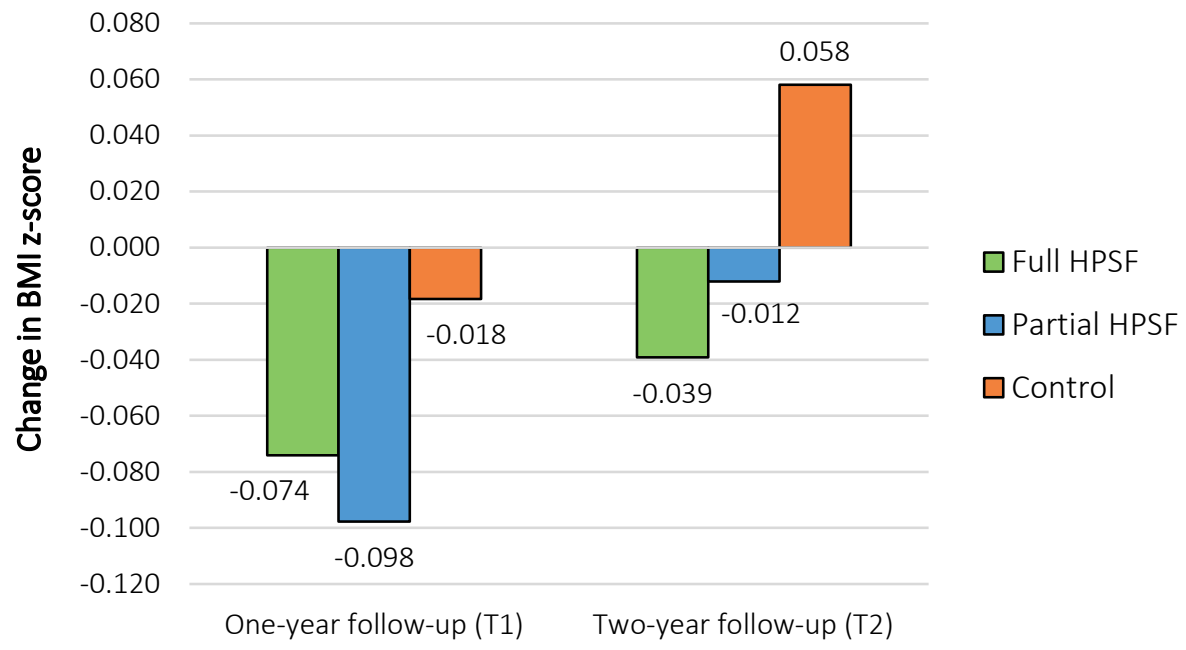

Figure 2. Observed change in children's BMI z-score at one year and two years' follow-up compared with baseline

Mixed model analyses were conducted to study the differences in effect among the three groups. The intervention effect was, compared with control schools, significant after one-year follow-up in the partial HPSF (ES=-0.05), not significant in the full HPSF (ES=-0.04) (Table 2). After two years' follow-up a significant intervention effect on children's BMI z-score was found in both versions of HPSF. Children's BMI z-score had decreased significantly more in the full HPSF $(E S=-0.08)$ and the partial HPSF $(E S=-0.07)$, compared with children of the control schools, whose estimated mean BMI z-score increased from baseline to two years as reported above. No significant difference in 
effect was found between the full and partial HPSF at T1 and T2. Both complete case analyses $(\mathrm{N}=759)$ and the sensitivity analyses in which children with an extremely low BMI z-score at baseline were excluded ( $\left.\mathrm{N}_{\text {excluded }}=14\right)$, resulted in comparable effect sizes. None of the interaction terms of the potential effect modifiers, i.e., gender, study year, SES, and weight status, was significant (Additional file 1, Table S1).

Table 2. One- and two-year estimated intervention effects on children's BMI z-score a

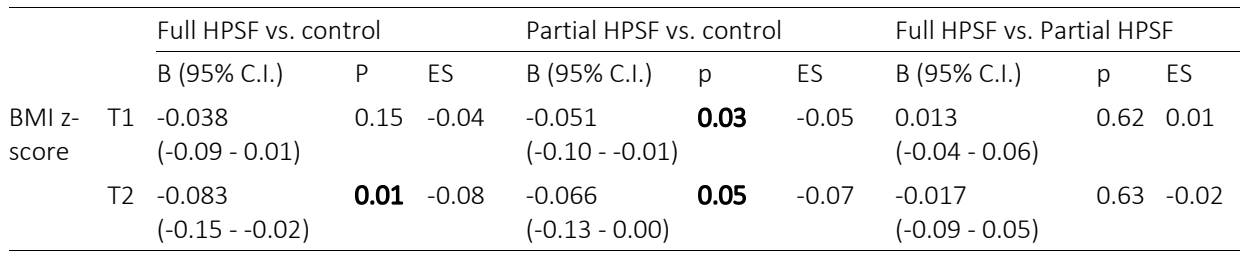

a Adjusted for baseline, gender, study year at TO, SES, and ethnicity. Bold $\mathrm{p}$-value = significant $(<.05)$ difference

Abbreviations: C.I. = confidence interval; $\mathrm{ES}=$ Effect size

\section{Discussion}

This study assessed the effects of HPSF on children's BMI z-score after one and two years' follow-up compared with children of control schools. The findings showed a favourable decreasing effect at T2 on children's BMI z-scores in both the full HPSF (standardized effect size $(E S)=-0.08$ ) and the partial HPSF (ES=-0.07) compared with control schools, where the BMI z-score actually increased at T2 compared with baseline. According to Lipsey's guidelines [100], these findings can be indicated as a small effect (effect size between 0 and 0.32). These small intervention effects are promising for three reasons: 1) they are already visible after two years of implementation, 2) they indicate a change in the increasing BMI trend observed in the control schools, and 3) they are slightly higher than the effect sizes found in several meta-analyses regarding school-based interventions $[108,109,117]$. The decrease in BMI z-score found in this study in the full and partial HPSF can therefore be considered as a favourable and promising intervention effect. No significant differences were found between the full and partial HPSF. The main distinction between them was the provision of a healthy lunch. However, the process evaluation of Bartelink et al. has shown that providing this lunch led to the implementation of additional health-promoting changes (e.g., health promoting policy, educational lunch) [110]. Additional health-promoting changes were not implemented in the partial HPSF [110]. However, since no significant differences were found between the full and partial HPSF, this might indicate that the differences between the two versions of HPSF did not have an additional favourable effect on the children's BMI z-score. 
The favourable effects on children's BMI z-scores seem to indicate that the children improved their health behaviours. Indeed, significant favourable intervention effects were found after one- and two-years' follow-up for the full HPSF on children's dietary behaviours for, among others, school water consumption and lunch intake of vegetables and dairy products [111]. Children's sedentary time and light PA significantly improved after two years' follow-up. Almost no significant favourable results on children's health behaviours were found in the partial HPSF. Since it is the co-existence and interaction of several nutrition and PA behaviours that results in a positive (or negative) energy balance and weight gain (or loss) [118, 119], the results suggest that many small improvements on several different health behaviours have occurred in the children of the partial HPSF, leading to the favourable effects on their BMI z-score.

Even though the effects of HPSF on children's BMI z-score seem promising, it is important to realize that two years' follow-up is too short to conclude that HPSF has led to sustainable changes. A longer follow-up period is needed to study whether the results found are not only due to the children's enthusiasm for and cooperation with the new changes in school, which might result in intervention effects that diminish after longer follow-up periods. This can be the reason for the smaller observed change scores after two years' follow-up compared to after one year, shown in Figure 2. On the other hand, the favourable results that are still found after two years' follow-up might indicate that new habits and routines have developed in children's health behaviours. The latter is not easy to change and requires a shift in the social norms of all people in the school regarding 'normal' health behaviours. Therefore, further research into HPSF should investigate its long-term effects on children's BMI z-score. Other outcomes should also be investigated to study the effects of HPSF, including children's educational achievements and well-being and the cost-effectiveness of the intervention. This broader scope of the effects of HPSF is included in the overall study design and will be investigated by our multi-disciplinary research group [55]. The specific focus in the current study enabled us to investigate the effects of HPSF on children's BMI z-score in much more detail.

The second research question investigated whether HPSF has different effects within specific subgroups of children. Effect-modification analyses showed no significant interactions at T1 and T2. However, effect sizes give a better indication since the big difference in group sizes in the subgroups of, for example, children's weight status, influenced the $p$-value. All effect sizes showed similar patterns to the overall analyses. These results seem to indicate that no specific subgroups of children were found to benefit more from HPSF, which is promising as often school-based interventions only seem effective for specific subgroups $[31,108]$. These results are especially promising when related to health inequalities, because even when interventions are successful in 
improving children's health, they may still increase health inequalities. This can happen when an intervention is of greater benefit to advantaged groups, e.g., high SES, than to disadvantaged groups, e.g., low SES [120]. Given the results of the effect modification analyses, HPSF can be seen as an example of an intervention that does not seem to increase health inequalities among children. Further research with longer follow-up periods should investigate whether HPSF contributes to reducing the health inequalities.

\section{Limitations and strengths}

The longitudinal quasi-experimental design can be seen as a limitation of this study, since we were unable to (cluster-) randomize schools. However, due to this design, we were able to test the effectiveness in terms of differences in children's BMI z-scores between the three school groups over time, and were also able to enrol schools on the basis of motivation, which reflects the real-life situation of school health promotion. Moreover, participants did not significantly differ from non-responders in the participating schools and other children in the region with regard to health and lifestyle [121].The lack of randomization could, however, have resulted in confounding bias. Therefore, we controlled for baseline BMI z-score, gender, study year at T0, SES score, and ethnicity in all analyses. The significant differences in children's BMI z-scores at baseline between the three groups could indicate that children in the control schools are less open to change: their habits in unhealthy behaviours are stronger as they have already led to overweight or obesity. On the other hand, this difference, which we controlled for, may have resulted in an underestimation of the effect: more room for improvement existed for the children in the control schools compared with the full and partial HPSF.

Next, HPSF seemed to affect all children in the intervention schools. However, a decrease in BMI z-score might not be favourable for all of them, for example when they already have an extremely low BMI z-score. Therefore, to ensure that the findings reflected the children for whom a decrease in BMI z-score is favourable, we conducted extra sensitivity analyses in which we excluded the children with extremely low BMI zscores at baseline. These analyses showed comparable results. The high number of children enrolled in the measurements, the low drop-out rate, and the objectively measured BMI were other strengths of this study. There were missing data because some participants did not participate from the start, other participants finished school before the last measurement period in 2017, the parental questionnaire was not completed, respondents skipped questions, or data could not be obtained due to the absence of the child. To deal with the missing data, multiple imputations were used, and a sensitivity analysis, in which only complete cases were included, was conducted. Complete case analysis showed similar results to the original analysis, which increased the reliability of the findings in this study. 


\section{Conclusions}

Taking all the results and limitations into account, it can be concluded that HPSF was effective in lowering children's BMI z-scores after one and two years' follow-up and no specific subgroups of children were found to benefit more from the intervention. Even though longer follow-up periods are needed to draw hard conclusions, both versions of the initiative seem promising in offering perspective in the on-going obesity epidemic in young children. 


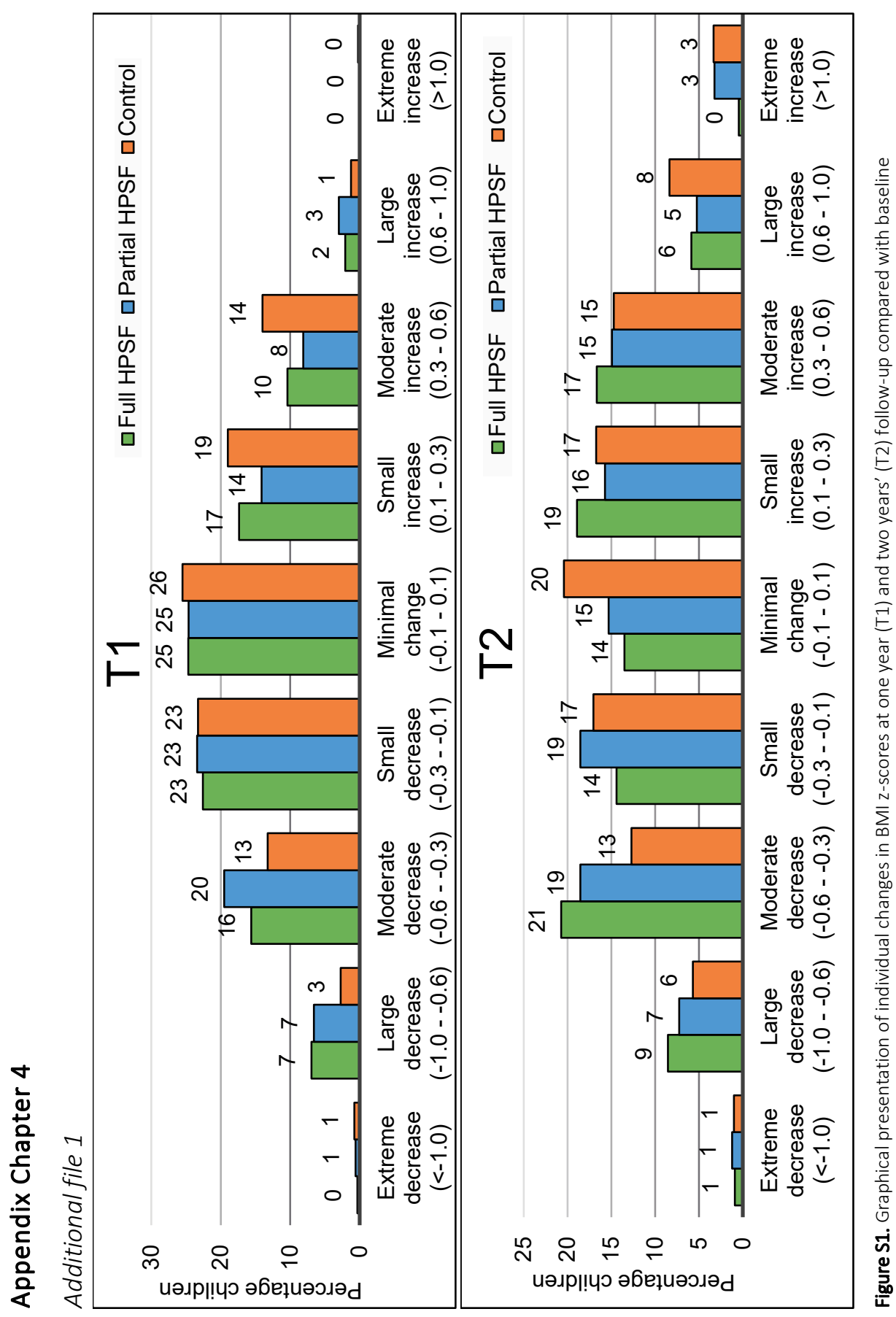




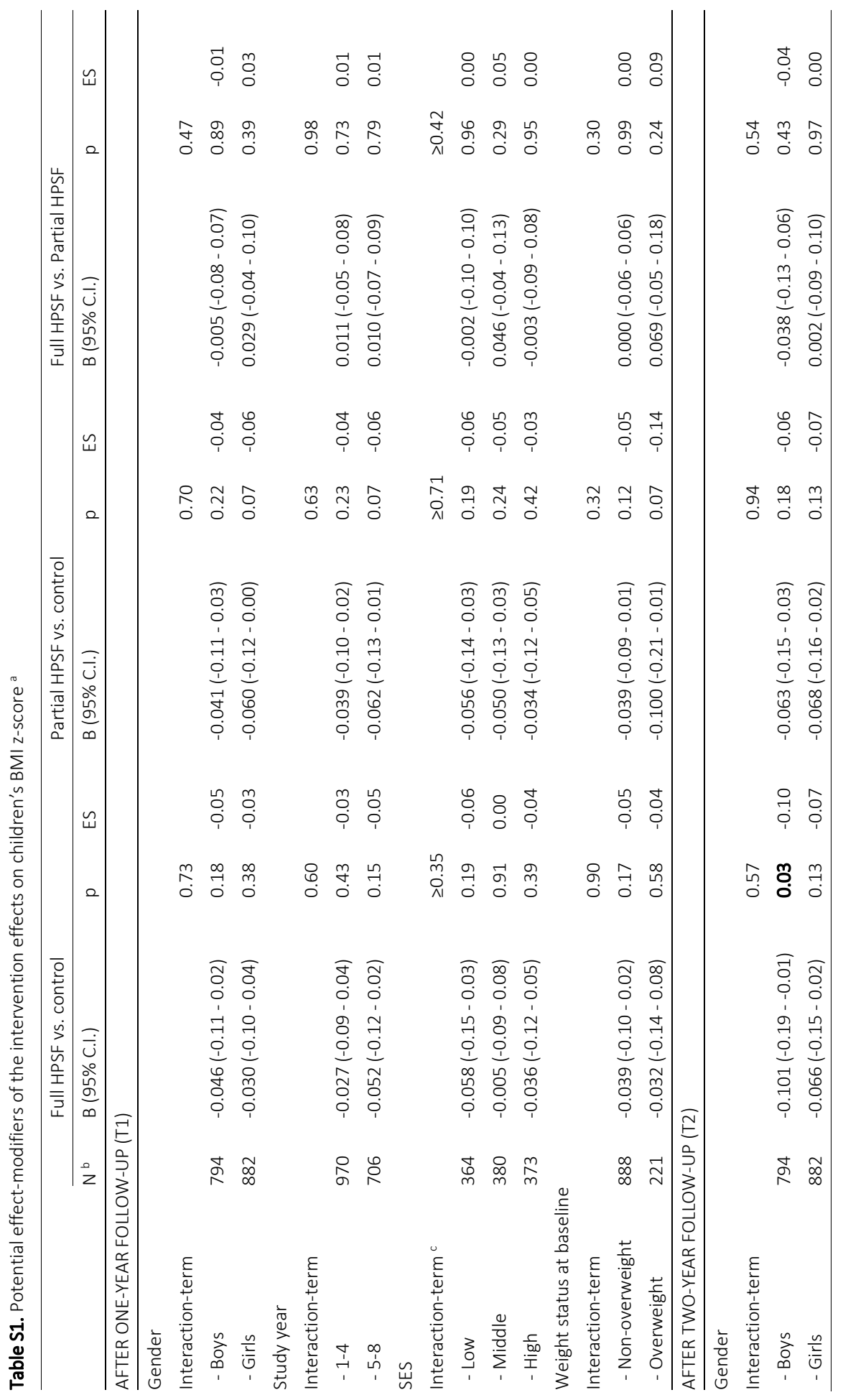




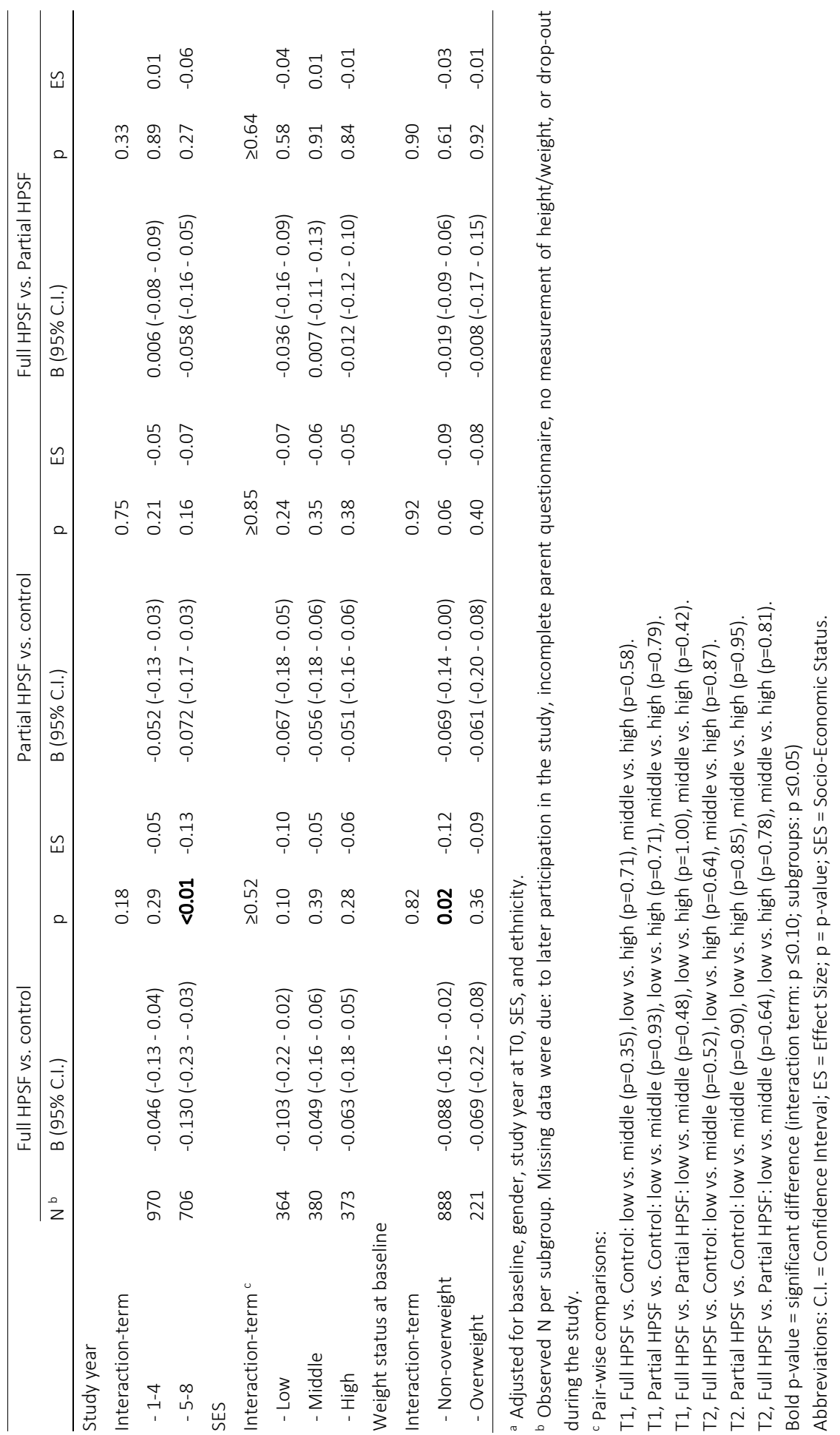





\section{Chapter 5}

One-and two-year effects of the Healthy Primary School of the Future on children's dietary and physical activity behaviours: A quasi-experimental study

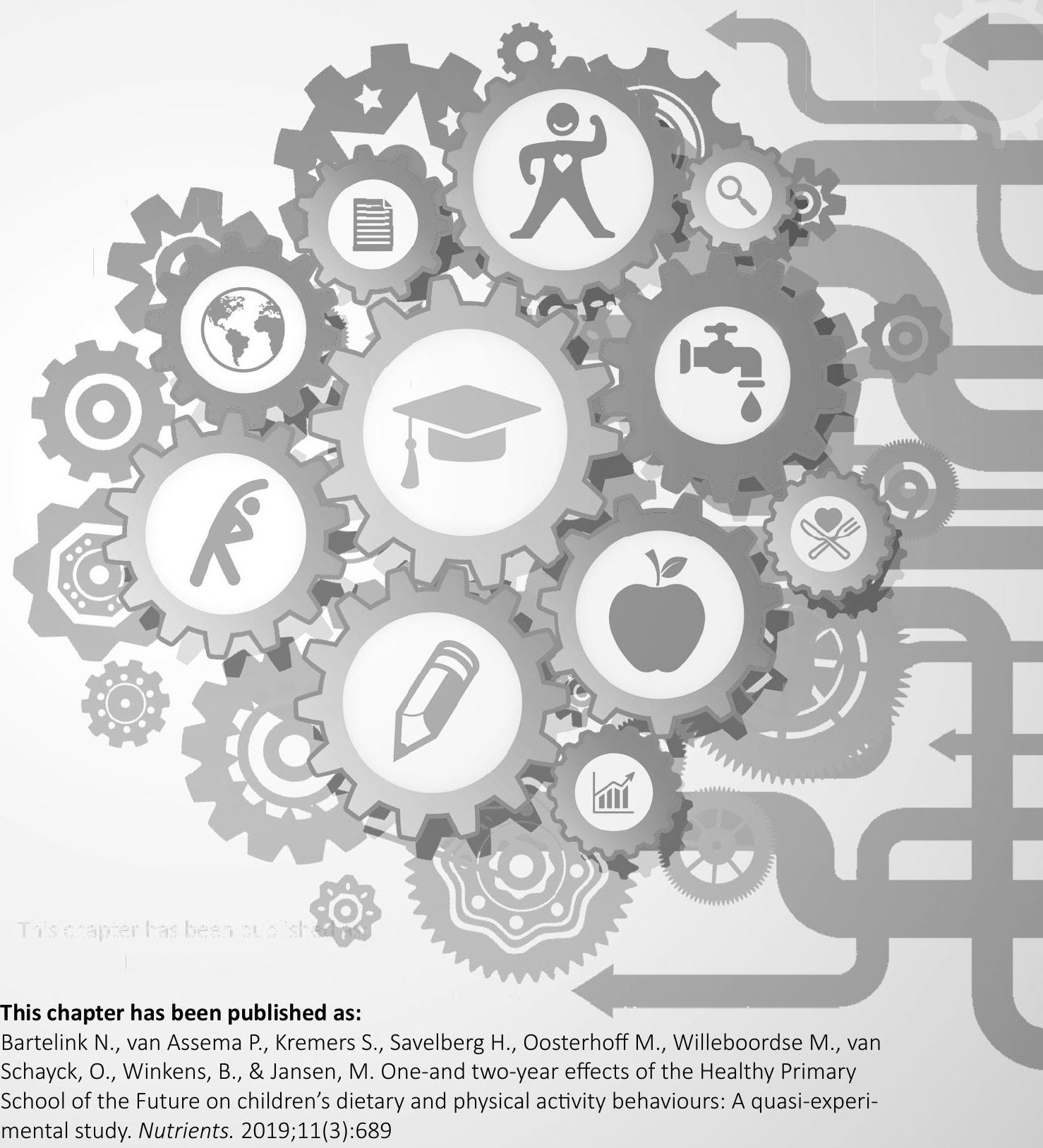




\section{Abstract}

Schools can help to improve children's health. The 'Healthy Primary School of the Future' (HPSF) aims to sustainably integrate health and well-being into the school system. This study examined the effects of HPSF on children's dietary and physical activity (PA) behaviours after 1 and 2 years' follow-up. The study ( $n=1676$ children) has a quasi-experimental design with four intervention schools, i.e., two full HPSF (focus: nutrition and PA) and two partial HPSF (focus: PA), and four control schools. Accelerometers and child- and parent-reported questionnaires were used at baseline, after 1 (T1) and 2 (T2) years. Mixed-model analyses showed significant favourable effects for the full HPSF versus control schools for, among others, school water consumption (effect size $(E S)=1.03(T 1), 1.14$ (T2)), lunch intake of vegetables (odds ratio $(O R)=3.17(T 1), 4.39(T 2))$ and dairy products $(O R=4.43(T 1), 4.52(T 2))$, sedentary time $(E S=-0.23(T 2))$, and light PA $(E S=0.22(T 2))$. Almost no significant favourable effects were found for partial HPSF compared to control schools. We conclude that the full HPSF is effective in promoting children's health behaviours at T1 and T2 compared with control schools. Focusing on both nutrition and PA components seems to be more effective in promoting healthy behaviours than focusing exclusively on PA. 


\section{Introduction}

Dietary and physical activity (PA) habits are formed at a young age [1], whereby unhealthy habits can already lead to overweight and obesity [2]. The health behaviours of children are suboptimal in Western countries, including the Netherlands: $42 \%$ of children (aged 4-9 years) consume at least $150 \mathrm{~g}$ of fruit per day, this percentage drops to $20 \%$ for 9-12 year olds. The prevalence of vegetable intake shows similar percentages: $41 \%$ of $4-9$ year olds and $25 \%$ of 9-12 year olds eats at least $150 \mathrm{~g}$ of vegetables per day [3]. Regarding PA, only half (48\%) of Dutch children (aged 4-12) meet the guidelines for PA of 60 min of moderate-to-vigorous physical activity (MVPA) per day [4]. Consequently, 13-15\% of Dutch children (aged 2-21 years) are overweight, and $1.8-2.2 \%$ are classified as obese, which is a two- to three-fold increase compared with 1980 [5]. Childhood overweight often tracks into adulthood [6] and is related to health and psychosocial problems, reduced quality of life, and higher health care costs [7-9]. An association also exists between health and educational achievement: Health status affects the capacity to learn, while educational achievements affect health status [11]. This link between health and education often results in persistent socioeconomic health inequity problems that continue to exist from generation to generation $[12,13]$.

Schools are increasingly recognized as significant in improving children's health behaviours since a large proportion of a child's day is spent there, and schools reach all children [14, 59]. However, school-based health interventions are often not integrated in the school system and are characterised by relatively low priority, a lack of coordination, and are often supply-driven, resulting in limited effects or effects that diminish in the long term [19, 25]. The Health-Promoting School (HPS) framework, initiated by the World Health Organization, aims for a whole-school approach, with a focus on reorienting school systems toward sustainable health promotion [21]. HPS focuses not only on classroom-based health education, but also on changes in school policy and the schools' physical and social environment, using bottom-up involvement of pupils, parents, teachers and staff. Several reviews have been published on the effectiveness of HPS in improving the health and well-being of school children $[30,91,117,122]$. Even though the findings indicate small favourable effects in terms of PA and healthier food choices, the reviews also reveal that the findings were not uniform across the included studies. Many studies showed suboptimal results, often due to a short duration of the intervention, a lack of a whole-school approach and implementation challenges $[20,63,123]$. Implementation challenges can be considered a result of the interaction between the intervention and the specific context $[32,42$, 47]. Therefore, various studies suggest revising the idea of interventions as something fixed or static, and considering them as 'events' occurring within the school system [54].

The 'Healthy Primary School of the Future' (HPSF) is a Dutch initiative based on the HPS framework (including, e.g., whole school approach, participation, partnerships) and 
embraces the contextual systems approach $[55,92]$. This initiative aims to sustainably integrate health and well-being within the school system.

The processes and effects of the initiative, implemented in four pilot schools, are being investigated in an overall study by a multi-disciplinary research group [55, 92]. The primary outcome of the overall study is children's BMI z-score, which significantly decreased after 2 years' follow-up in the HPSF schools compared to control schools [124]. The current study focuses on two key aspects of HPSF, i.e., healthy nutrition and PA. Recent research suggests that by addressing two clustered health behaviours, a spill-over or synergistic effect might occur, whereby the probability of enhancing one health behaviour increases when an individual has successfully changed the other health behaviour $[125,126]$. This means that, for example, an increase in physical activity may lead to improved eating behaviours and vice versa. Therefore, simultaneously addressing healthy nutrition and PA might be more effective due to the facilitation of this potential synergistic effect.

The aim of the current study is to examine the effects of HPSF on children's dietary and PA behaviours after 1 and 2 years' follow-up compared with control schools, with two schools focussing on both nutrition and PA (full HPSF), and two schools focussing only on PA (partial HPSF). We hypothesized that in the full HPSF, effects will be noted on both dietary and PA behaviours, and in the partial HPSF mainly on PA behaviours. Additionally, we hypothesized that larger effects will be found in the full HPSF, due to the potential synergy between dietary and PA behaviours in children.

\section{Methods}

\section{Study design}

The current study has a longitudinal quasi-experimental design with four intervention schools (two full and two partial) and four control schools, which maintained the school curriculum that is currently common practice in the Netherlands [55]. Inclusion criteria for schools include being a member of the educational board 'Movare', since they were one of the initiators of HPSF, and a minimum of 140 children in the study years two till five, to be able to study the effects of HPSF with enough power. The schools are all situated in the Parkstad region in the southern part of the Netherlands. This region has a low average socioeconomic status (SES), and unhealthy behaviours and overweight are highly prevalent compared with the rest of the Netherlands [39, 40]. Ethical approval (14-N-142) for the overall study was obtained from the Medical Ethics Committee Zuyderland located in Heerlen (Parkstad, the Netherlands). All participants were required to complete an informed consent form, signed by (both) parents. All four intervention schools started implementation of HPSF in November 2015. Funding for implementation is provided until the end of 2019. However, the four schools have 
committed to continued implementation after 2019 and make the changes sustainable in their school. Measurements in all eight included schools were conducted in September-November of 2015 (T0), 2016 (T1) and 2017 (T2); the overall study continues until 2019. The data that support the findings of this study were collected as part of the 'Healthy Primary School of the Future' quasi-experimental study. Data collection will take place until 2019 to study the effects after 4 years of exposure. Following article publication, data will become available on the 4-year effects and other potentially comparative studies in the Netherlands. A detailed description of the overall study and the recruitment of the schools is reported in Willeboordse et al. [55]. The study was retrospectively registered in the ClinicalTrials.gov database on 14 June 2016 (NCT02800616).

\section{The Healthy Primary School of the Future}

Three collaborating organizations, i.e., the regional educational board 'Movare', the regional Public Health Services and Maastricht University, developed the HPSF initiative. The initiative is based on the principles of the HPS framework and aims to sustainably integrate health and well-being within the whole school system. To achieve this aim, HPSF intends to establish a broad collaboration between school, parents, and external partners, which should lead to a co-creation movement in schools. This includes topdown and bottom-up processes to develop and implement together health-promoting changes in all aspects of the school system, e.g., school's physical and social environment, school's health policy, education, and school routines. This also refers to sustainability research, whereby, among other things, partnership, ownership, organizational routines, and add-in changes, are important factors for success [40, 127, 128].

On top of the HPS framework, the aim was to create some form of positive disruption in the school, by initiating two changes top-down: (1) a free healthy lunch each day (only in full HPSF) and (2) structured PA sessions after lunch. These changes are contextualized bottom-up and should lead to momentum for bottom-up processes to institutionalise health-promoting routines in the school. The time for having lunch (in the full HPSF) was increased to 20-30 min. The total lunch break time in these schools was prolonged by about $60 \mathrm{~min}$. For this reason, the school day was extended: Children of the full HPSF attend school to approximately 15:30/15:45 instead of 15:00. A dietician of the caterer developed a lunch menu cycle that changed every 10 weeks, in which at least $80 \%$ of the products met the advice of the Dutch Health Council [112]. A mid-morning snack, consisting of fruits and/or nuts, was also provided. The lunch, a bread-based cold meal, was typically Dutch. During lunch break time, the children participated several times a week in structured PA sessions; one or two times per week they could participate in cultural activities. The PA sessions were carried out in the schoolyard and, when available and needed, in parks, forest, and/or sports hall in the 
neighbourhood. All schools collaborated with sport clubs or other external partners to offer specific activities as well. Since the two changes were contextualized bottom-up, this resulted in some differences between schools in the form of the changes; the content remained comparable. To not increase the workload of teachers even further, the top-down changes were implemented by external pedagogical employees provided by childcare organizations. This integration of the childcare organization during school hours is not to provide a temporary solution, but to change the school's organization in a sustainable way. The aim for the future is to bring school and childcare more together and thereby create an integrated day for children, whereby children are supervised by the same people prior, during and after school hours. The abovementioned commitment of schools and childcare organizations to continued implementation, also includes this employment of external pedagogical employees during school hours. Employees of sports and leisure organizations supported the pedagogical employees during implementation when needed, and after a year they provided a training course ( 8 sessions of $2 \mathrm{~h}$ ) to supply them with additional tools for how to motivate children for active participation during the PA sessions.

The implementation of the lunch and the duration of the lunch break time were the main differences between the two versions of HPSF. The full and partial HPSF implemented the structured PA sessions in a comparable way and had quite similar support from external partners [110]. Both the full- and partial-HPSF schools involved teachers and parents in the adoption decision and the process of adapting the two changes into the school context. All four intervention schools used a children voice group, with representatives from each class in school, to get insight into the opinion of children regarding HPSF. In this way, the experiences of children were being heard and the changes could be further contextualized to fit better to the children's needs and wishes. Differences existed in the implemented additional health-promoting changes [110]. The full HPSF improved their health policy, provided water bottles to all children, and provided an educational lunch once a week. The partial HPSF did not implement additional health-promoting changes. Each of the four intervention schools selected a teacher as school coordinator, who managed HPSF in their school. Overarching the four schools, the HPSF initiative was led by a project leader from Movare and an executive board with representatives of the three collaborating organizations, including the project leader. A project team was created with representatives of all partners involved: the four schools, Movare, regional Public Health Services, Maastricht University, the Limburg provincial authorities, childcare organizations, the caterer, and sports and leisure organizations. 


\section{Study population}

All children (age 4 to 12) and their parents from the eight schools ( $n=2326$ at T0) were invited to participate in the study; no inclusion or exclusion criteria were set. This included children from study year one to eight, which is comparable to 2 years of Kindergarten and six grades. Recruitment was done via information brochures for parents. In addition, the research team visited the classrooms to inform children about the study and encourage them to ask their parents to participate. Due to the dynamic population in the schools (new children enter and other children finish school each year), we focused in this study only on the children who were enrolled in the schools at baseline till the end of this 2-year study. In this way, only children were included in the current study who participated in the full 2 years of HPSF in their school. The group of children included in this study were: At baseline (TO), children from study year one to seven; at T1, children from study year two to eight; and at T2, children from study year three to eight. Children who joined the study at T1 or T2 were included, even though no baseline data were available. Even though these children joined the study after 1 or 2 years, they were at baseline already participating in their school and thus also exposed to HPSF during the full 2 years of this study. Children who switched to other schools between $\mathrm{TO}$ and $\mathrm{T} 2$ were excluded.

\section{Data collection procedures}

In each school, the data were gathered during 1 week of measurements. Inter-rater variability was minimised by training researchers according to a strict protocol.

\section{Accelerometers}

At the beginning of the measurement week, all participating children from study year two to eight received an accelerometer for 7 days (Actigraph GT3X+, $30 \mathrm{~Hz}, 10 \mathrm{~s}$ epoch). The monitor was attached to the hip with an elastic band and had to be worn all day except while sleeping or during activities in which water was involved (e.g., swimming, bathing, and showering). To control for the influence of weather on PA levels, data on weather conditions between 6 a.m. and 11 p.m., e.g., mean temperature, sun exposure and precipitation, were collected from the Royal Dutch Meteorological Institute (KNMI).

\section{Questionnaires}

Children and one of their parents were asked to fill out one (parents) or two (children) questionnaires. The child questionnaires were based on the validated parent questionnaire, but simplified to make it appropriate for children. We did not validate these adapted questionnaires. However, all questionnaires were pretested, for difficulty, length and content by experts in the field of health promotion, the target group, e.g., individual children and parents, and classes of children in a primary school. 


\section{Parent questionnaire}

A digital questionnaire for parents was used to obtain information about, among others, the education level and country of birth of both parents, household income, and children's health behaviours. To assess children's PA behaviours, 14 questions were used from the Local and National Youth Health Monitor [129]. These questions in the monitor were based on the International Physical Activity Questionnaire, which has acceptable validity [130]. Parents were asked how many days a week and how many minutes a day their child engaged in several PA activities (e.g., active transport, leisure time PA indoors and outdoors, and sports clubs) and sedentary activities (e.g., watching television, computer use, and social media use) during the past week. Twelve questions from the Local and National Youth Health Monitor were used to assess children's dietary behaviours [131]. These questions were based on the short Fat List, which has acceptable validity [132]. Parents were asked about the number of days (on a scale from 0-7 days a week) their child consumed breakfast; ate warm vegetables, salads or raw vegetables, and fruits; and consumed water and sugar-sweetened beverages (soft-, sports-, and energy-drinks) during the past week. They were also asked how many times a week their child ate the following four snack types: chocolate, salted snacks, cookies, and soft ice-creams (on a scale of 0-7 days a week). All parents of participating children (study years one to eight) received the questionnaire. It took about $60 \mathrm{~min}$ for the parents to fill in as other aspects were also explored, such as quality of life. Parents had approximately 1-3 months to fill in the questionnaire: From the start of each measurement week until the end of the calendar year. Two reminders were sent in this period if the questionnaire was not yet completed.

\section{Child questionnaire}

The questionnaire was filled in by children of study years four to eight and was used to assess their dietary behaviours and their water consumption specifically in school. Questions regarding the children's PA behaviours mainly focused on whether they liked specific activities and were not used in the current study. Twelve questions were included, based on the Local and National Youth Health Monitor, regarding daily breakfast intake, the intake of fruit and vegetables, the consumption of water (at school), sugar-sweetened beverages (soft-, sports-, and energy-drinks), and the consumption of the four snack types (chocolate, salted snacks, cookies, and soft icecreams) [131]. The reply options were simplified to (1) never or almost never, (2) sometimes (1-3 days per week), (3) often (4-6 days per week), and (4) every day; the reply for daily breakfast intake was yes/no. The questionnaire was filled out by hand during class hours in the presence of at least one member of the research team. It took about 40 min to fill out, as other aspects such as quality of life were also included. 


\section{Child lunch questionnaire}

The questionnaire regarding children's lunch intake was filled out by children of study years three to eight and consisted of recall questions $(n=8)$ with yes/no reply options regarding the consumption of food types that are included in the Wheel of Five designed by The Netherlands Nutrition Centre [133], i.e., bread, cereals, butter, cheeses, fruits, vegetables, milk/yoghurt, and water during lunch that day. The questionnaire was filled out by hand immediately after lunch time, which took about 5 minutes.

\section{Measures}

\section{Covariates}

Children's gender, age and study year at baseline were collected via the database of the educational board Movare. SES was calculated as the mean of standardized scores on maternal education level, paternal educational level, and household income (adjusted for household size) [113]. SES scores were categorized into low, middle and high based on tertiles. Children's ethnicity was determined by the country of birth of both parents and divided into (1) Western background (including the Netherlands) and (2) nonWestern background [114]. If one of the parents was born in a non-Western country, the child's ethnicity was assigned to non-Western. Body Mass Index (BMI) was assessed by anthropometric measurements of height and weight [55]. BMI z-scores were calculated using Dutch reference values [5].

\section{Outcomes}

\section{Children's PA behaviours}

PA levels derived from the accelerometry data were processed using ActiLife version 6.13.3 (ActiGraph, Pensacola, FL, USA). Wear time validation was assessed using Choi's classification criteria [134]. Minimal wear time was defined as $480 \mathrm{~min}$ per day between 6 a.m. and 11 p.m. [135]. The first day of measurement was excluded to prevent reactivity [136]. Measurements containing at least three weekdays (after excluding the first measurement day) and one weekend day were used in the analyses [137]. Mean temperature, sun exposure and precipitation were merged with the accelerometry data to obtain weather scores for all days the child wore the accelerometer. The activity levels in counts-per-minute (CPM) were classified using Evenson's cut-off points [138]: sedentary behaviour (SB; $\leq 100$ CPM), light PA (LPA; 101-2295 CPM), and moderate to vigorous PA (MVPA; $\geq 2296$ CPM). The children's total time spent on PA and sedentary behaviours was derived from the parent questionnaire. The number of days per PA behaviour (active transport, leisure time PA inside and outside, and sport clubs) or sedentary behaviour (watching TV, using computer, social media use) were multiplied by the average number of minutes spent in a day and divided by seven (active transport was divided by five). The four specific PA behaviours were summed into a PA behaviours 
total score, and the three sedentary behaviours were summed into a sedentary behaviours total score. Missing values were imputed using a child's mean imputation if there were not too many items missing (scales with $<5$ items: max 1 item missing; scales with $\geq 5$ items: $\max 2$ items missing), otherwise they were considered as missing PA behaviour.

\section{Children's dietary behaviours}

Total scores for healthy and unhealthy dietary behaviours were used, due to the high number of dietary outcomes, and the fact that small changes in several specific dietary behaviours could be better detected by using total scores. A total score for healthy behaviours was calculated by the mean number of days (parent-reported) and the mean score (child-reported) of breakfast consumption, intake of fruits, vegetables (parent-reported: distinction between warm and cold), and water. A total score for unhealthy behaviours was calculated by the mean number of days (parents) or mean score (child) of intake of sugar-sweetened beverages and the four different snack types. To be able to include breakfast intake in the child-reported total score, this score had to be recoded to (1) not every day and (3) every day. Missing values were imputed using a child's mean imputation if there were not too many items missing $(<5$ items: max 1 missing; $\geq 5$ items: $\max 2$ missing). The variable 'school water consumption' (range: 0 (never)-3 (every day)) of the child questionnaire was used to assess children's water intake in school in particular.

\section{Children's lunch intake}

The following six food types were derived from the child lunch questionnaire: fruits, vegetables, grains, dairy, water, and butter. The items bread and cereals were combined into the food type grains, and milk/yoghurt and cheese were combined into the food type dairy. To give an indication of the nutritional value of children's lunch, we summed the six different food types consumed and created a dichotomous variable to study whether children consumed at least two of the food types during lunch. Additionally, to investigate if change occurred in the consumption of specific combinations of food types, we created five variables for the most common combinations, e.g., grains and fruit, grains and vegetables, dairy and fruit, dairy and vegetables, and grains and dairy.

\section{Statistical analyses}

Data were analyzed using IBM SPSS Statistics for Windows (version 23.0, IBM Corp, Armonk, NY, USA). Pearson's chi-square tests and ANOVA tests were conducted to analyze the comparability of observed participant characteristics at baseline, i.e., gender, study year, SES status, ethnicity, BMI z-score, and PA and dietary behaviours, among the full HPSF, the partial HPSF, and control schools. The percentage of children 
who improved in a specific behaviour after 1 and 2 years, i.e., changed in a favourable direction compared with their baseline result, was studied by descriptive statistics. Linear mixed model analyses were used to assess the longitudinal intervention effects on children's PA levels and behavioural outcomes; Generalized Estimating Equations were used for binary outcomes. Since measurements were repeated, within participants we used a two-level model with measurements as the first level and participants as the second level. The fixed part of the model consisted of group (full HPSF, partial HPSF, control), time (T0, T1, T2) and the interaction terms of group with time. We were not able to include class as a level in the model, because commonly more than one division of a class existed, e.g., $4 a$ or $4 b$, and children often did not have fixed class divisions for all years. All analyses were adjusted for the covariates: gender, study year at baseline, SES, ethnicity, and children's BMI z-score at baseline. The analyses regarding children's PA levels were also adjusted for weather conditions (mean temperature, sun exposure in hours/day, and precipitation in hours/day). Missing data, including missing data at baseline, were imputed using a multiple imputation method with fully conditional specification (FCS) and 10 iterations, generating 50 complete datasets. BMI z-score, gender, study year at baseline, school type, ethnicity, SES score, temperature, sun exposure, and precipitation were used to obtain a complete covariate set, with a likelihood-based approach being used for missing outcome variables. This latter was done for practical reasons as the number of outcome variables was too large. A two-sided $p$-value $\leq 0.05$ was considered statistically significant. Standardized effect sizes (ES) were determined for numerical outcomes, which were computed as pooled estimated mean difference divided by the square root of the pooled residual variance at baseline. Binary outcomes resulted in odds ratios.

\section{Results}

At baseline (TO), 2326 children and their parents were invited to participate in the overall study to investigate the effects of HPSF; $60.3 \%$ joined the study ( $n=1403$ ). Because of the study's dynamic population, a total of 1974 children and their parents participated in the study within the 2-year follow-up period (data collected at one timepoint at least). Due to the selection used for the current study, i.e., only including the children who were in study years one to seven at baseline and excluding school switchers, we included 1676 children in the analyses. This selection and the study's flow diagram are similar to the study that investigated the 1- and 2-year effects of HPSF on children's BMI $z$-score [31]. Of these children, $47.4 \%$ were boys, the mean age was 7.5 years old, and $94.1 \%$ had a Western ethnicity. In the full HPSF, 537 children were included, in the partial HPSF, 478 children, and in the control schools, 661 children. No covariates differed significantly at baseline between the three school groups, except for BMI $z$-scores $(p=0.034)$ : children in the control schools $(B M I-z=0.232)$ had a higher 
mean BMI $z$-score compared with children of the full HPSF $(B M I-z=0.051)$ and the partial HPSF (BMI- $z=0.092)$. Regarding children's dietary and PA behaviours, many significant differences existed at baseline, with unhealthier behaviours mostly found in the children in the control schools compared to the full and partial HPSF (Tables 1-3). Not all parents filled out the parent questionnaire: Parents of 1115 children (66.5\%) completed the questionnaire at least once. The child questionnaire was filled out at least once by $96.1 \%$ of the children, the child lunch questionnaire by $98.3 \%$ of the children. Sufficient accelerometer data, i.e., enough wear time to be included in the analyses, in at least one measurement was reached in $81.5 \%$ of the children.

\section{Children's PA behaviours}

Significant favourable intervention effects were found in the accelerometry data in the full HPSF versus control schools (Table 1). The percentage time spent sedentary had decreased more (ES $=-0.23$ ) and the percentage time spent in light PA had increased more $(E S=0.22)$ at T2 in children of the full HPSF compared with control schools. More than a quarter of all children (28.2\%) improved, i.e., decreased their sedentary time at T2 in the full HPSF, which was more than the percentage of children in the control schools (21.6\%). The percentage time spent in MVPA did not differ significantly in the full HPSF compared with control schools. However, the percentage of children who improved their time spent in MVPA was higher in the full HPSF (44.4\%) than the control schools (35.8\%). The parent-reported data regarding children's PA behaviours showed mixed results: The total time per day spent on both PA behaviours (ES $=-0.22$ ) and sedentary behaviours ( $E S=-0.29$ ) had decreased more at T2 in the full HPSF compared with control schools. In the partial HPSF, no significant intervention effects were found in the accelerometry data or parent-reported data compared with control schools (ES between -0.07 and 0.08 ).

\section{Children's dietary behaviours}

Significant favourable intervention effects were found for parent-reported children's dietary behaviours in the full HPSF. Children's healthy dietary behaviours (total score for breakfast, fruit, vegetables, and water) improved significantly more in the full HPSF compared with control schools at T1 $(E S=0.20)$ and T2 (ES $=0.19)$ (Table 2). Effect sizes per item of this total score were largest for water consumption (Additional file 1, Table S1). Children's unhealthy dietary behaviours decreased significantly more for the full HPSF versus control schools at T1 $(E S=-0.23)$. A significant favourable intervention effect was also found for child-reported water consumption at school: at T1 and T2, a significantly higher increase was found in children of the full HPSF compared with control schools (T1: ES = 1.03; T2: ES = 1.14). More than three-quarters of all children improved, i.e., increased their water consumption at school at T1 and T2 in the full HPSF, which was almost double the percentage of children compared with the control 
schools. In the partial HPSF, no significant intervention effects were found for parentreported children's dietary behaviours compared with control schools (ES between -0.14 and 0.07). Results on child-reported unhealthy dietary behaviours showed a significant favourable intervention effect at T2: a significantly larger decrease in the partial HPSF compared with control schools ( $E S=-0.25)$.

\section{Children's lunch intake}

Significant intervention effects were found for children's lunch intake (child-reported) in the full HPSF: A significantly higher increase was found at $\mathrm{T} 1$ for the consumption of fruit $(O R=2.63)$, vegetables $(O R=3.17)$ and dairy products $(O R=4.43)$ compared with control schools (Table 3). These higher increases remained significant at T2 for the consumption of vegetables $(O R=4.39)$ and dairy products $(O R=4.52)$. The consumption of grains and butter during lunch decreased significantly more at $\mathrm{T} 1$ (grains: $O R=0.43$; butter: $O R=0.22$ ) and T2 (grains: $O R=0.45$; butter: $O R=0.19$ ) in the full HPSF compared with control schools. The consumption of at least two food types during lunch increased significantly more in the full HPSF compared with control schools $(\mathrm{OR}=3.51(\mathrm{~T} 1)$ and $2.98(\mathrm{~T} 2))$. The consumption of five common food type combinations improved by approximately $30-40 \%$ at $\mathrm{T} 1$ and $\mathrm{T} 2$ in the full HPSF. In contrast, this percentage was much less in the control schools (8-20\%) (Additional file 2 , Table S2). In the partial HPSF, the consumption of vegetables (OR $=0.58$ ), dairy products $(O R=0.45)$ and butter $(O R=0.64)$ during lunch significantly decreased more at $\mathrm{T} 2$ compared with control schools. 


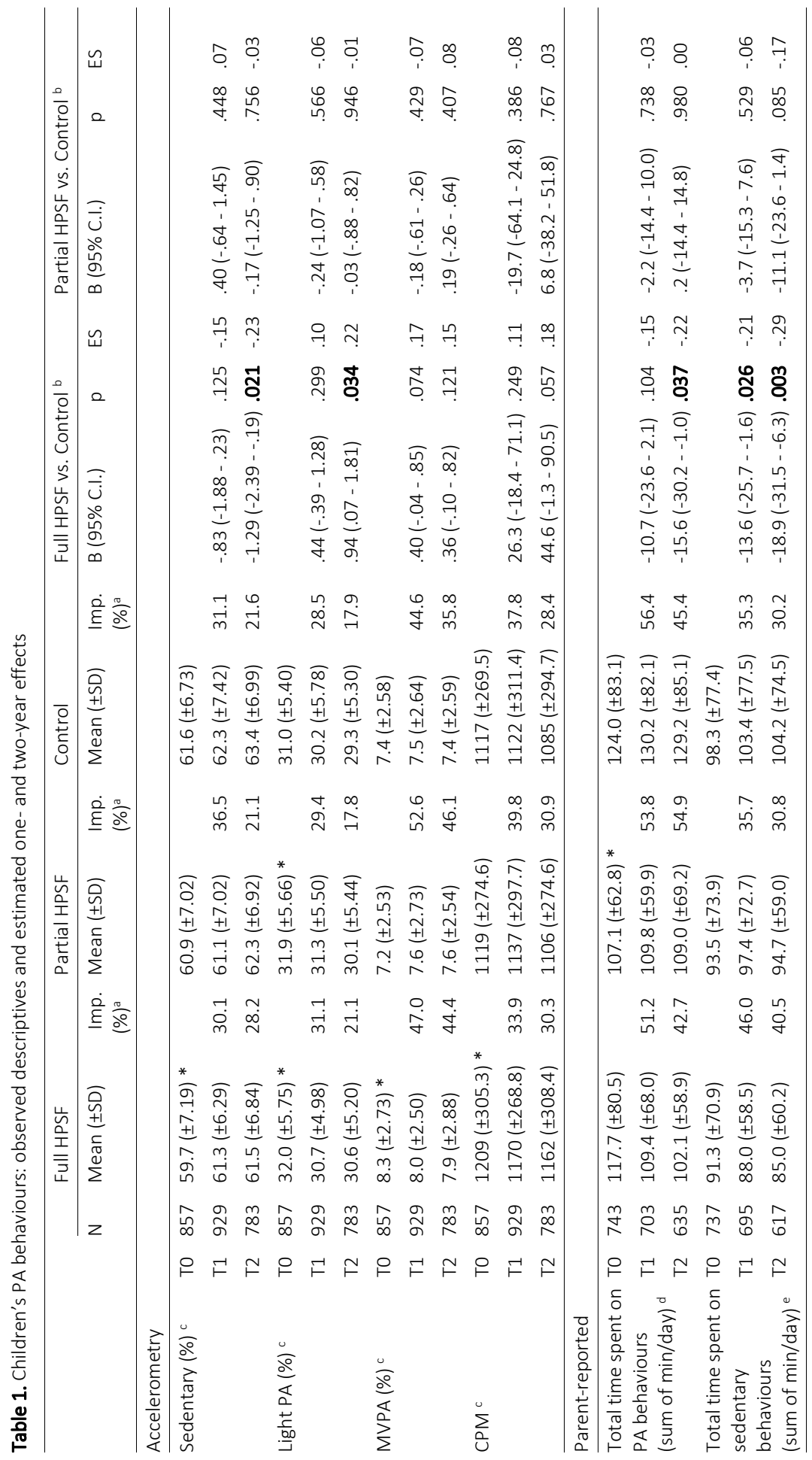


Effect of HPSF on children's dietary and PA behaviours

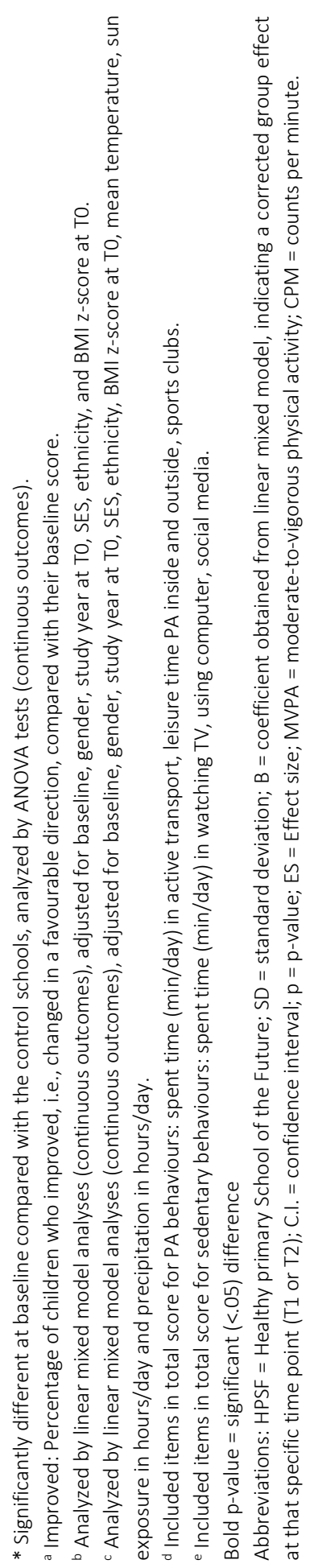




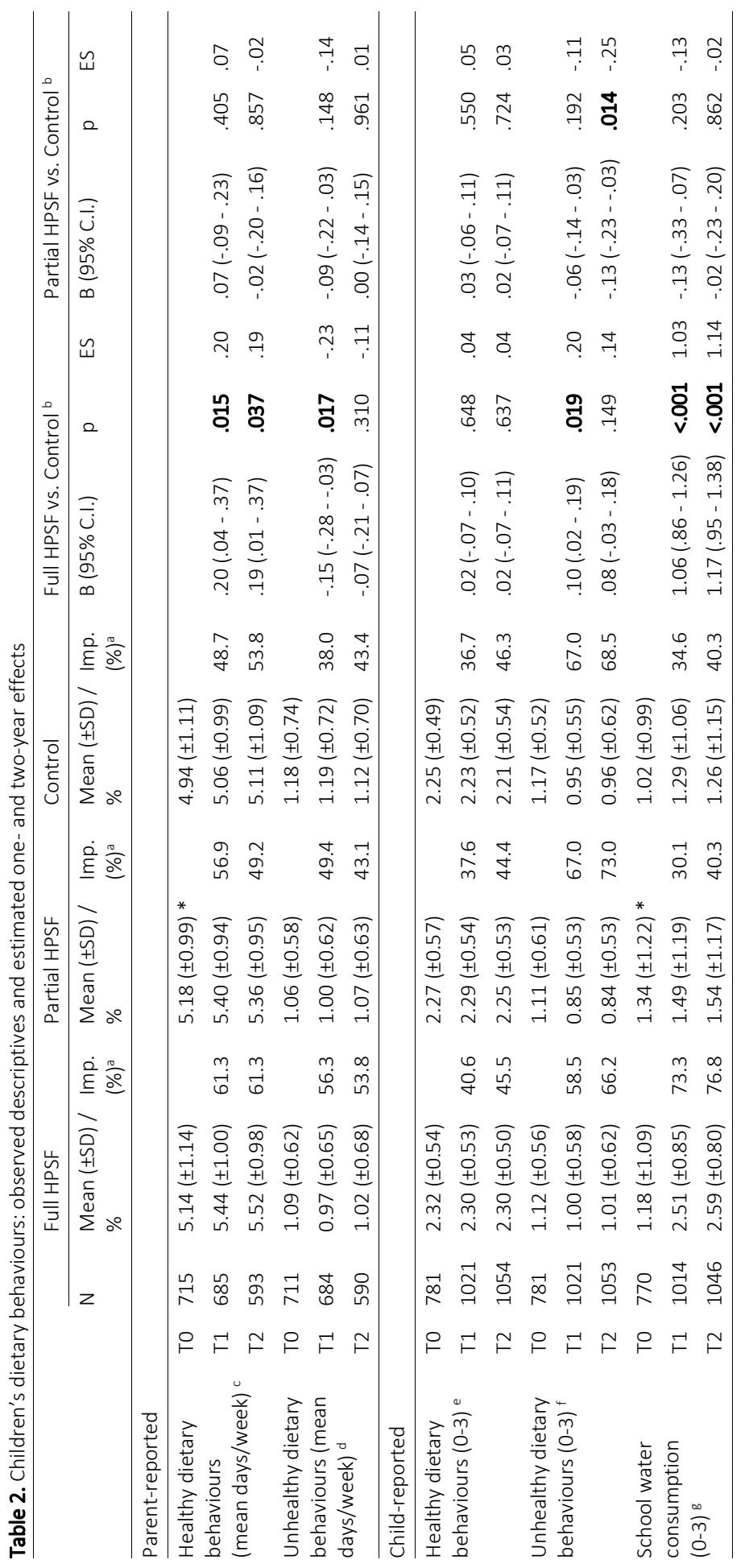


Effect of HPSF on children's dietary and PA behaviours

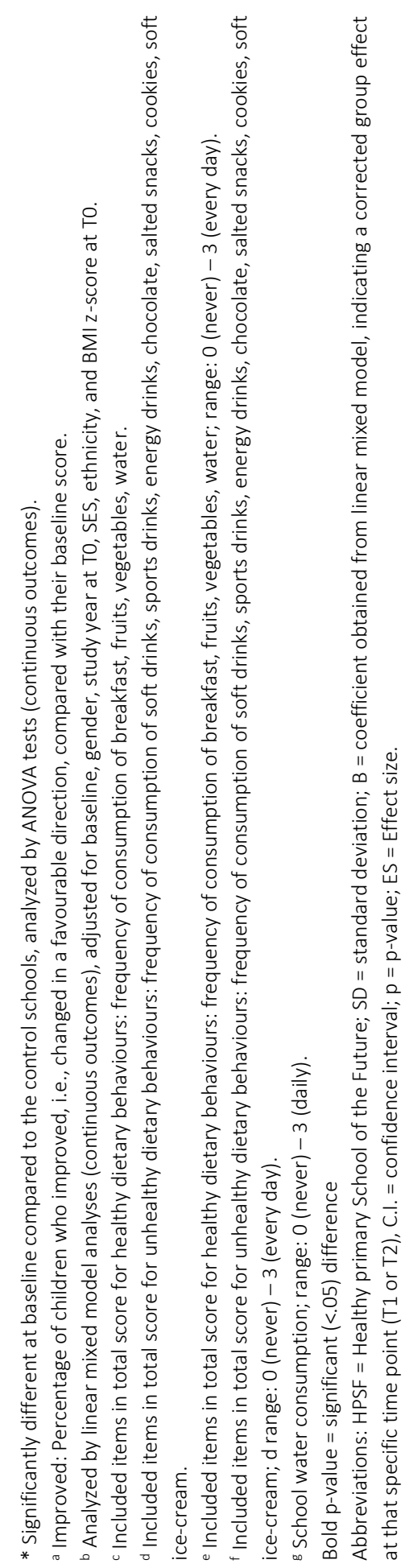




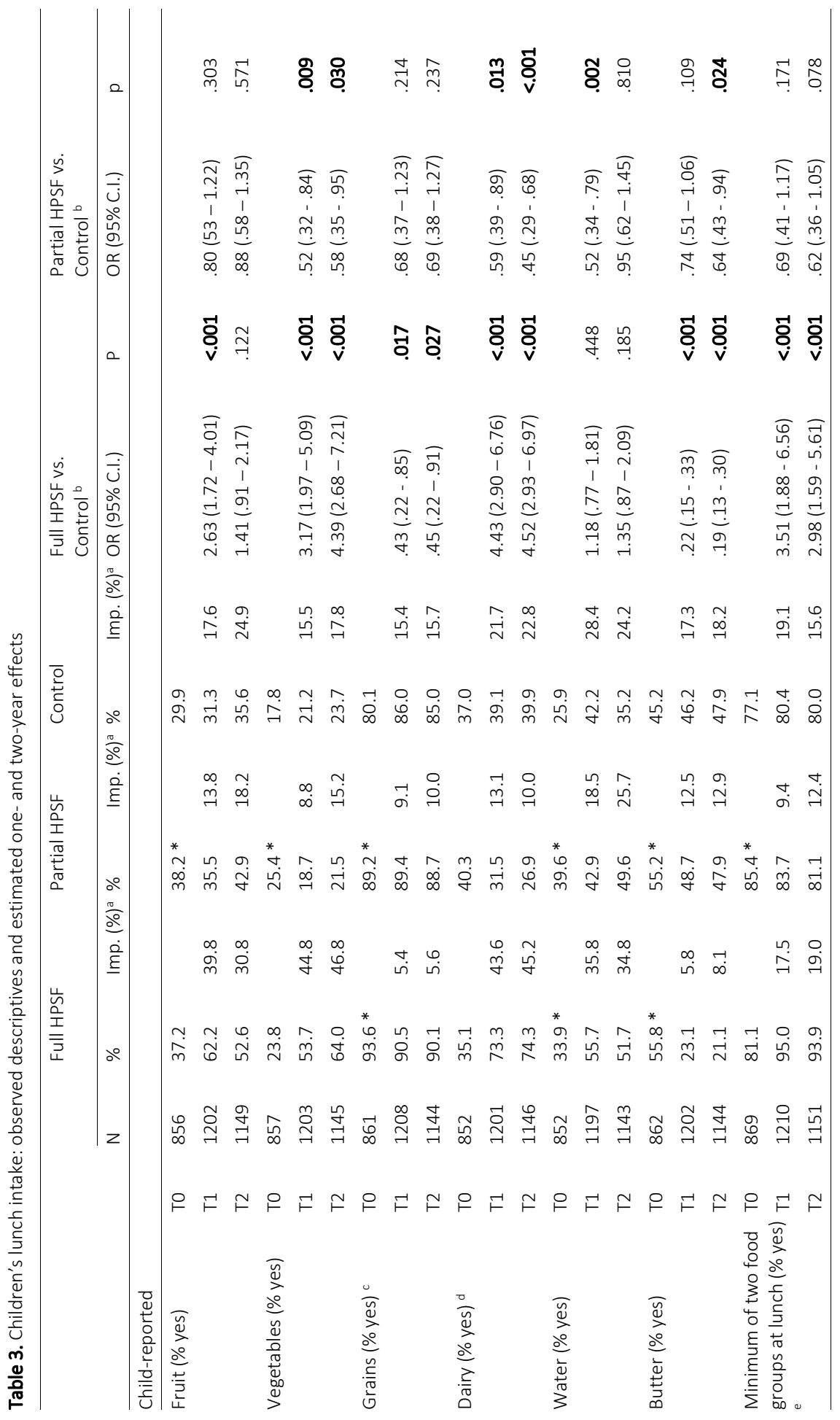


Effect of HPSF on children's dietary and PA behaviours

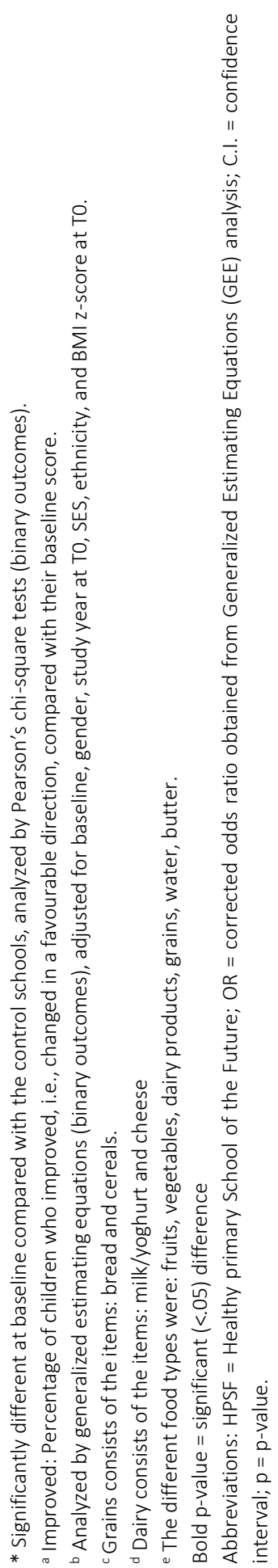




\section{Discussion}

HPSF is a health-promoting school initiative that uses a contextual systems approach $[21,55,92]$. The initiative aims to create health-promoting changes in different aspects of the school system, i.e., school's physical and social environment, school's health policy, education, and school routines. On top of the HPS framework, the aim was to create some form of positive disruption in the school, which should lead to momentum for bottom-up processes to institutionalise health-promoting routines in the school. The aim of the current study was to examine the effects of HPSF on children's dietary and PA behaviours after 1 and 2 years' follow-up compared with control schools. Favourable intervention effects on children's dietary and PA behaviours were found for the full HPSF. In contrast, almost no significant favourable results were found for the partial HPSF, where we expected favourable effects on children's PA behaviours. These effects are in line with the findings of the review of Langford et al., who investigated comparable school-based initiatives [122]. This review stated as well that PA behaviours significantly improved only in the initiatives with a focus on both healthy nutrition and PA behaviours, and not in initiatives that focused solely on PA behaviours. In contrast to our study, this review did not find any significant results on behaviours related to healthy nutrition for the schools with a focus on both healthy nutrition and PA. However, comparison is limited, since we used total scores and this review used fruit and vegetable intake and fat intake as outcomes.

The results found in the full HPSF regarding the accelerometry data can be seen as small intervention effects according to Lipsey's guidelines for effect sizes (small (00.32), medium (0.33-0.55) and large (>0.55)) [100]. The significant effects on children's sedentary time and light PA, but not on MVPA, are in line with other studies of schoolbased initiatives $[139,140]$. Contrary to the accelerometry-data, parent-reported data showed in the full HPSF not only a favourable effect (decrease in sedentary behaviours), but also an adverse effect (decrease in PA behaviours). These differences in effects found by using the two methods might be explained because assessing PA by subjective parent-reported questionnaires has a lower validity than objective accelerometry [141]. However, the differences might also be due to the focus of the PA-related questions for parents being outside of school hours, while the accelerometers assessed PA over the whole day. Children of the full HPSF have less time for sedentary and PA behaviours outside of school because of the extended school day. Since both behaviours decreased, it does not necessarily mean that the extra PA at school resulted in children compensating for PA outside of school hours, which has been found in other studies [142]. More in-depth research is needed to investigate the difference in effect during and outside of school hours on children's PA behaviours.

The large favourable intervention effect on school water consumption in the full HPSF is probably a result of implementing additional health-promoting changes related to water, e.g., handed out water bottles to all children and improved their school water 
policy, which created a more health-promoting environment and policy in the school. Both are important aspects of the HPS framework. Both schools referred to the momentum in the school created by the lunch to implement these water-related changes [110].

Furthermore, the increase in the consumption of fruits, vegetables and dairy products, and the decrease in grains and butter in the full HPSF seem to indicate that children eat more different food types during lunch. Their lunch intake seems to have changed from a typical Dutch bread-based lunch to a more diverse lunch. The large favourable intervention effect on the intake of at least two food types during lunch seems to validate this conclusion.

The intervention effects in the full HPSF were quite similar at both time points, and the T2 intervention effects were even higher for children's PA behaviours than the T1 intervention effects. This seems to indicate that the effects are not only due to the children's enthusiasm for and cooperation with the new changes in school, but that new habits and routines may have developed in the children's health behaviours. However, longer follow-up periods are needed to investigate the long-term effects.

The main difference between the full and partial HPSF was the implementation of the lunch, the duration of the lunch break time, and the implementation of additional health-promoting changes. However, the two versions of HPSF also had many similarities: both implemented the structured PA sessions in a comparable way, and they were quite similar in the coordination of HPSF and the support of external partners. Nonetheless, the full HPSF was more effective than the partial HPSF, also regarding children's PA behaviours. Three possible explanations can be given. First, as hypothesized, simultaneously addressing nutrition and PA seemed to create a synergistic effect that led to greater effectiveness. Various studies have indeed suggested that dietary and PA behaviours are associated and that the probability of enhancing a second behaviour, e.g., PA, increases when an individual has successfully changed a first behaviour, e.g., healthy nutrition $[125,126]$. Second, both the full and partial HPSF used a contextual systems approach and included top-down and bottomup processes to create health-promoting changes in the school [92]. Since the two topdown changes were also contextualized bottom-up, this resulted in some differences between schools in the form of the changes, e.g., assigning external pedagogical employees to a specific activity or to a specific class. The content of the changes remained comparable, however. Moreover, the results of the process evaluation of Bartelink et al. indicated that the lunch turned out to be a positive disruption in the full HPSF that created momentum for more bottom-up processes, including more involvement and support of teachers and parents, and it has led to additional healthpromoting changes (e.g., health-promoting policy) [110]. The partial HPSF did not implement the lunch, which resulted in limited bottom-up processes and no additional health-promoting changes in these schools. Due to this lack of additional changes, the whole school approach as suggested by the HPS framework is limited, which might 
explain the differences in effect between the full and partial HPSF. Third, the partial HPSF did not extend the lunch break time, whereas the full HPSF created a longer break by extending it by approximately $60 \mathrm{~min}$. Consequently, the time for the structured PA sessions was longer in the full HPSF compared with the partial HPSF.

Although we hypothesized that differences in effect would exist between the full and partial HPSF, we did not expect that in the partial HPSF no effects on children's PA behaviours would be found at all. An explanation for this absence might be that children's PA behaviours in the specific weeks of measurements were not representative of the children's PA behaviours in general. Moreover, the effects on children's PA behaviours might also be too small to detect. The results of the study regarding the effects of HPSF on children's BMI z-score found a small but significant decrease in BMI z-score after 2 years in both the full $(E S=-0.08)$ and partial $(E S=-0.07)$ HPSF [124], which suggests that also in the partial HPSF, some changes have occurred in the children's health behaviours [58]. Many small improvements on several different health behaviours can lead to a decrease in BMI z-score, since it is the co-existence and interaction of specific nutrition and PA behaviours that results in a positive (or negative) energy balance and weight gain (or loss) [118, 119].

\section{Limitations and Strengths}

The longitudinal quasi-experimental design can be seen as a limitation of this study, since we were unable to (cluster-) randomize schools. However, due to this design, we were able to test the effectiveness in terms of differences in children's health behaviours between the three school groups over time, and were also able to enrol schools on the basis of motivation, which reflects the real-life situation of school health promotion. However, due to no randomization, it has probably resulted in significant baseline differences between the three groups. The baseline differences in BMI z-scores and health behaviours seem to indicate that children in the control schools have developed stronger habits in unhealthy behaviours, which have already led to more overweight or obesity. These stronger habits can be more difficult to change, but also show more room for improvement for the children in the control schools compared with the full and partial HPSF, which can result in an underestimation of the effects. To deal with the limitation of no randomization, we controlled in all analyses for BMI Zscore at TO, gender, study year at TO, SES score, and ethnicity. Moreover, a methodological strength of the study is the objectively measured PA levels, all collected in the same season, and the matching of all measurements in the same week.

In addition to the abovementioned methodological limitation regarding assessing PA behaviours among parents, the use of questionnaires in general had its limitations as these are subjective measurements, which may lead to socially desirable answers [143]. Therefore, we used different data sources to obtain information about the children's health behaviours. The advantage of using questionnaires for children was that a high 
response rate (child questionnaire: 96.1\%, lunch questionnaire: 98.3\%) could be achieved by classical inquiry; children are often more honest in their answers and children's behaviours during the whole day can be assessed [144]. By pretesting, we made adjustments for age and improved the clarity of the questionnaires. However, these child-appropriate questions lead to less detailed data. The advantage of using a parental questionnaire was that more detailed questions could be asked. However, only children's behaviours outside of school hours can be assessed by them, and the response rate was much lower (66.5\%).

\section{Conclusions}

In the current study, we were able to investigate the effectiveness of the full and partial HPSF compared to control schools regarding children's health behaviours after 1 and 2 years' follow-up. Taking all results and limitations into account, we conclude that the full HPSF is effective in promoting children's health behaviours at T1 and T2 compared with control schools. Focusing on both nutrition and PA components seems to be more effective in promoting healthy behaviours than focusing exclusively on PA. 


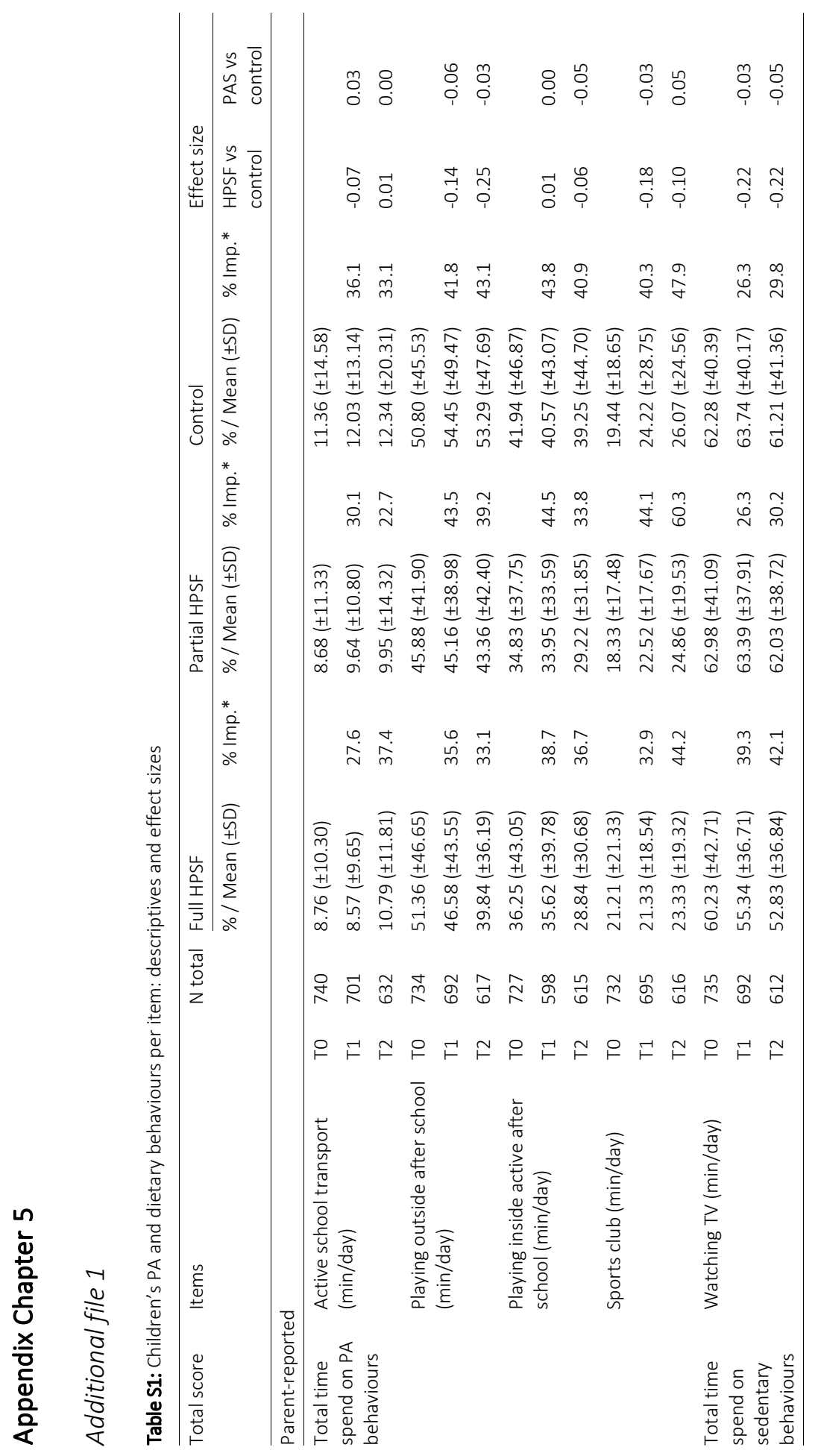




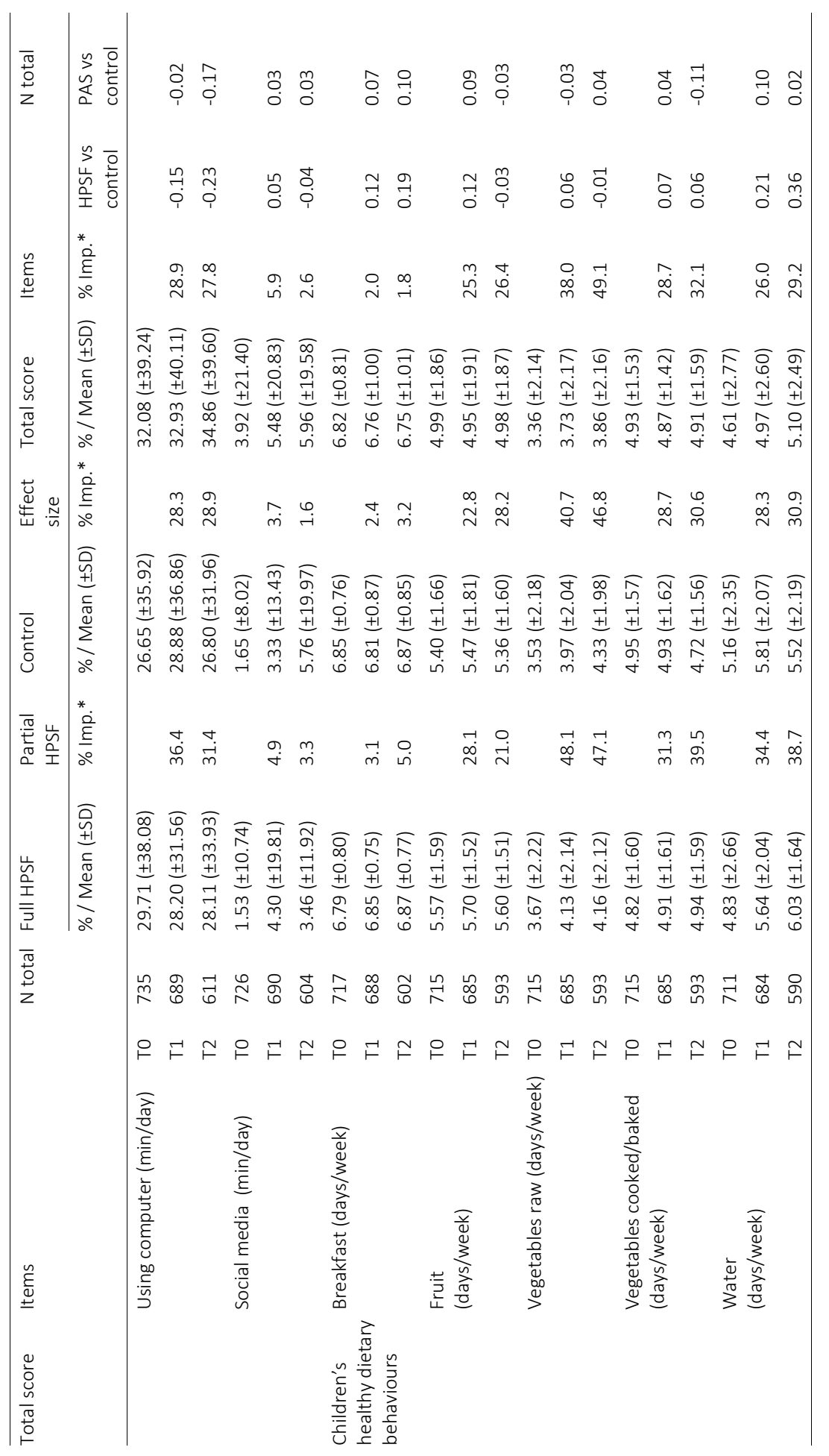




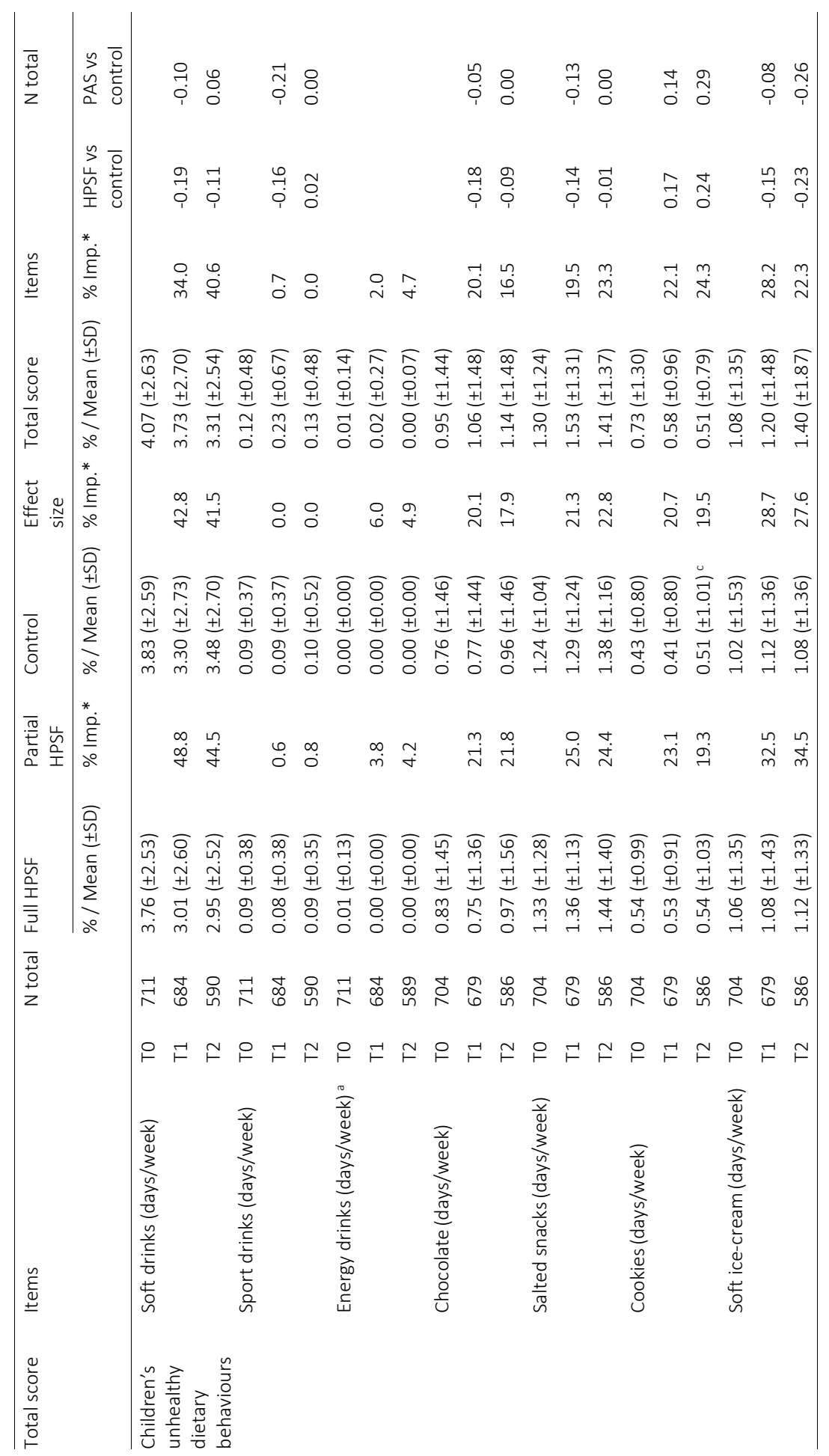




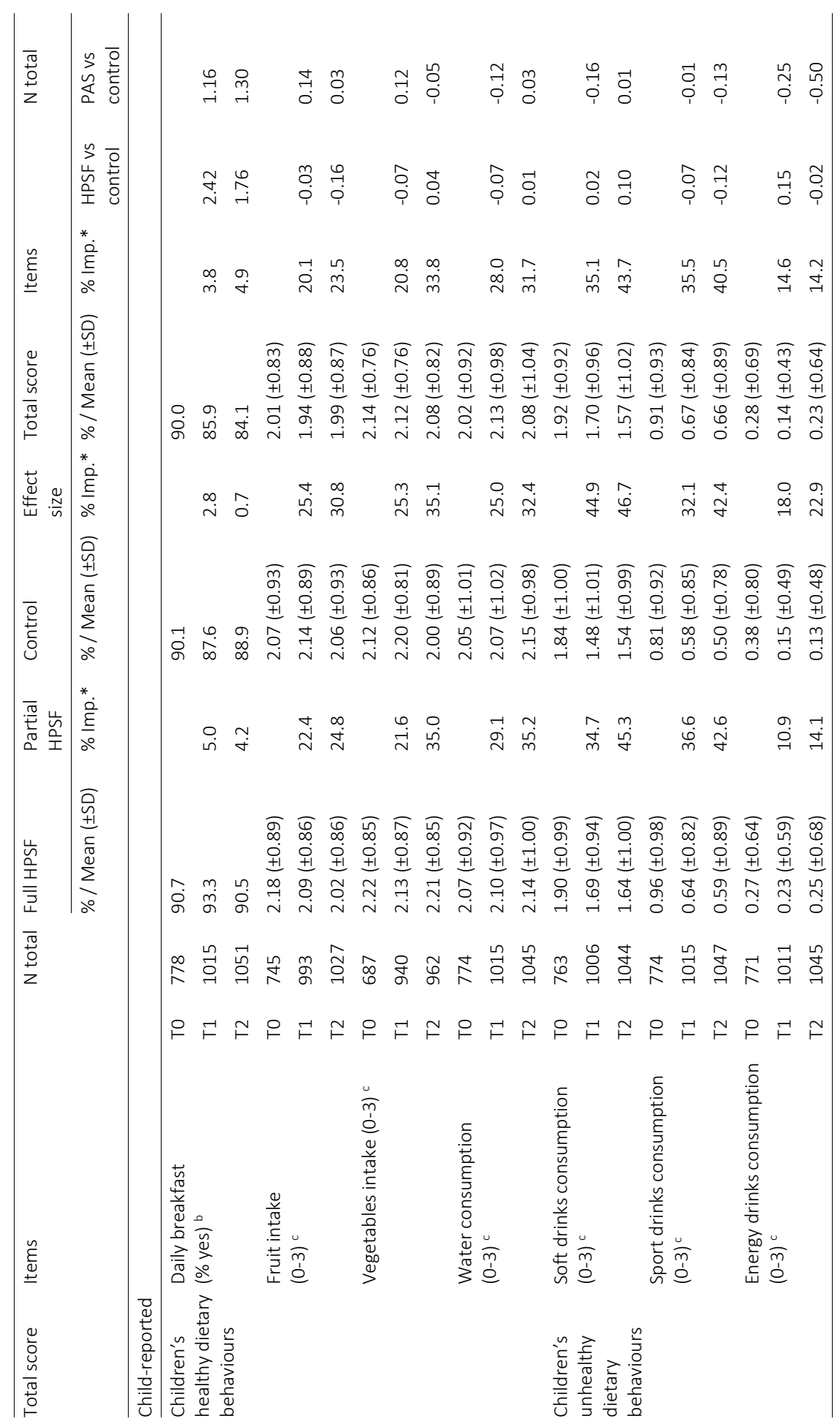




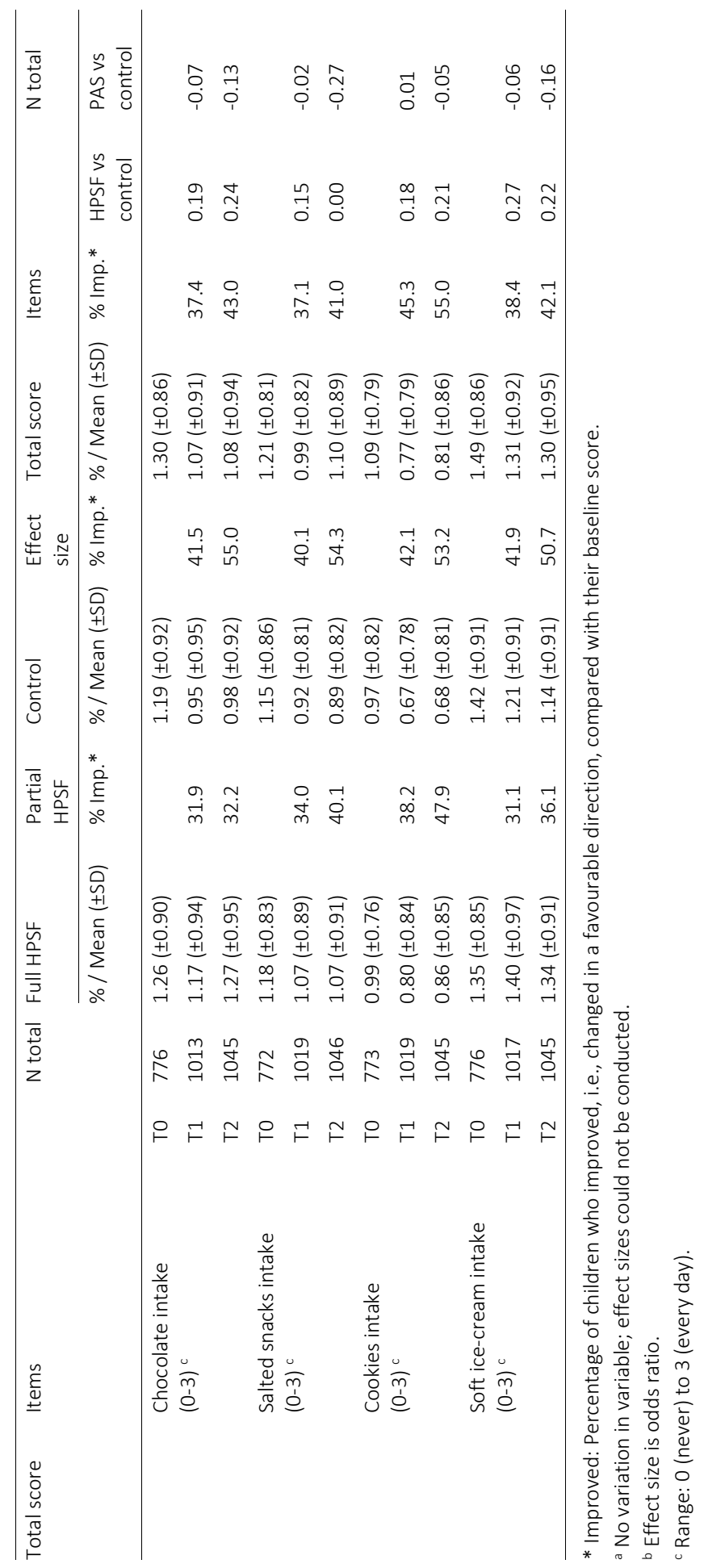


Effect of HPSF on children's dietary and PA behaviours

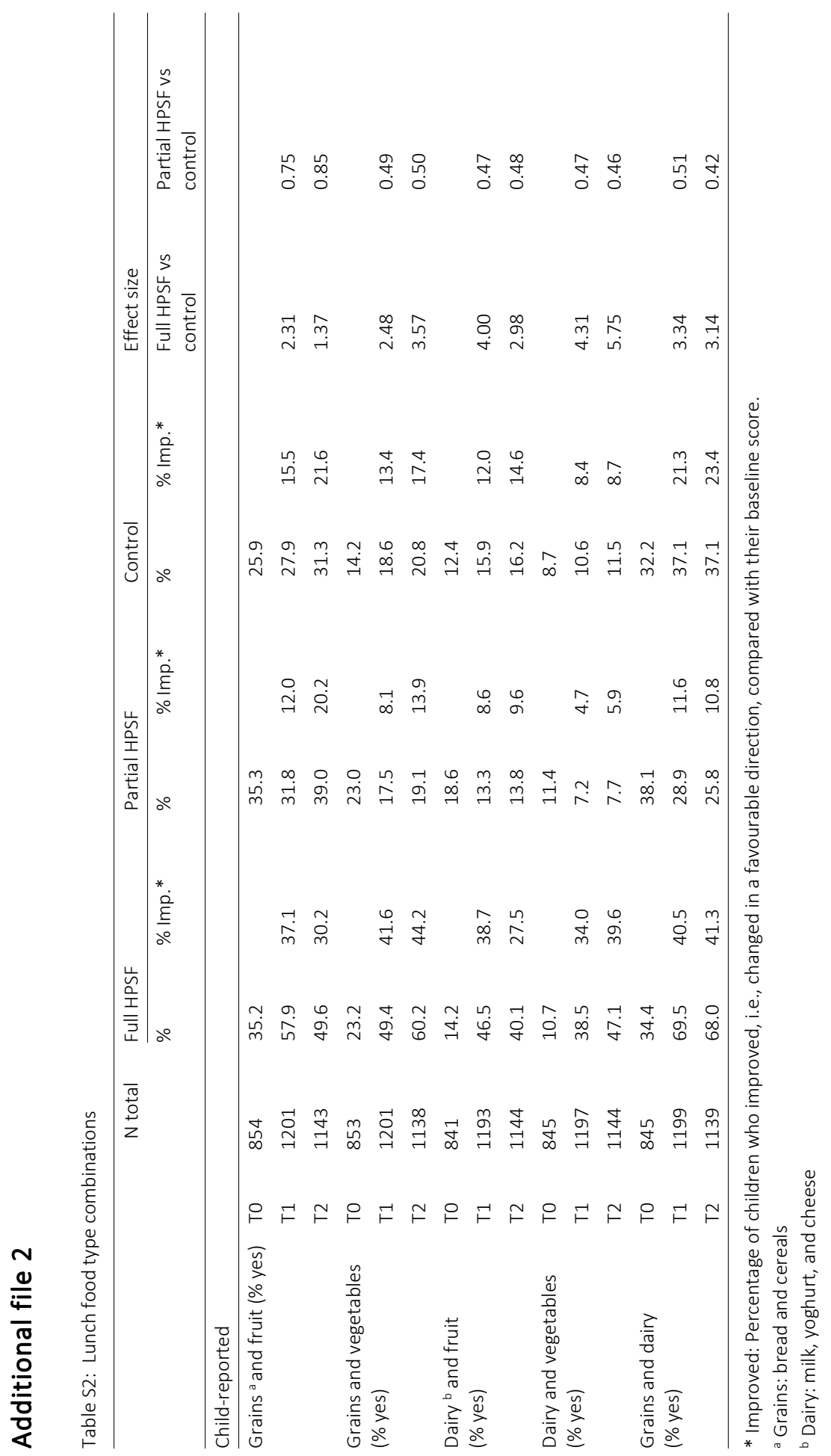





\section{Chapter 6}

Unravelling the effects of the Healthy Primary School of the Future: For whom and where is it effective?

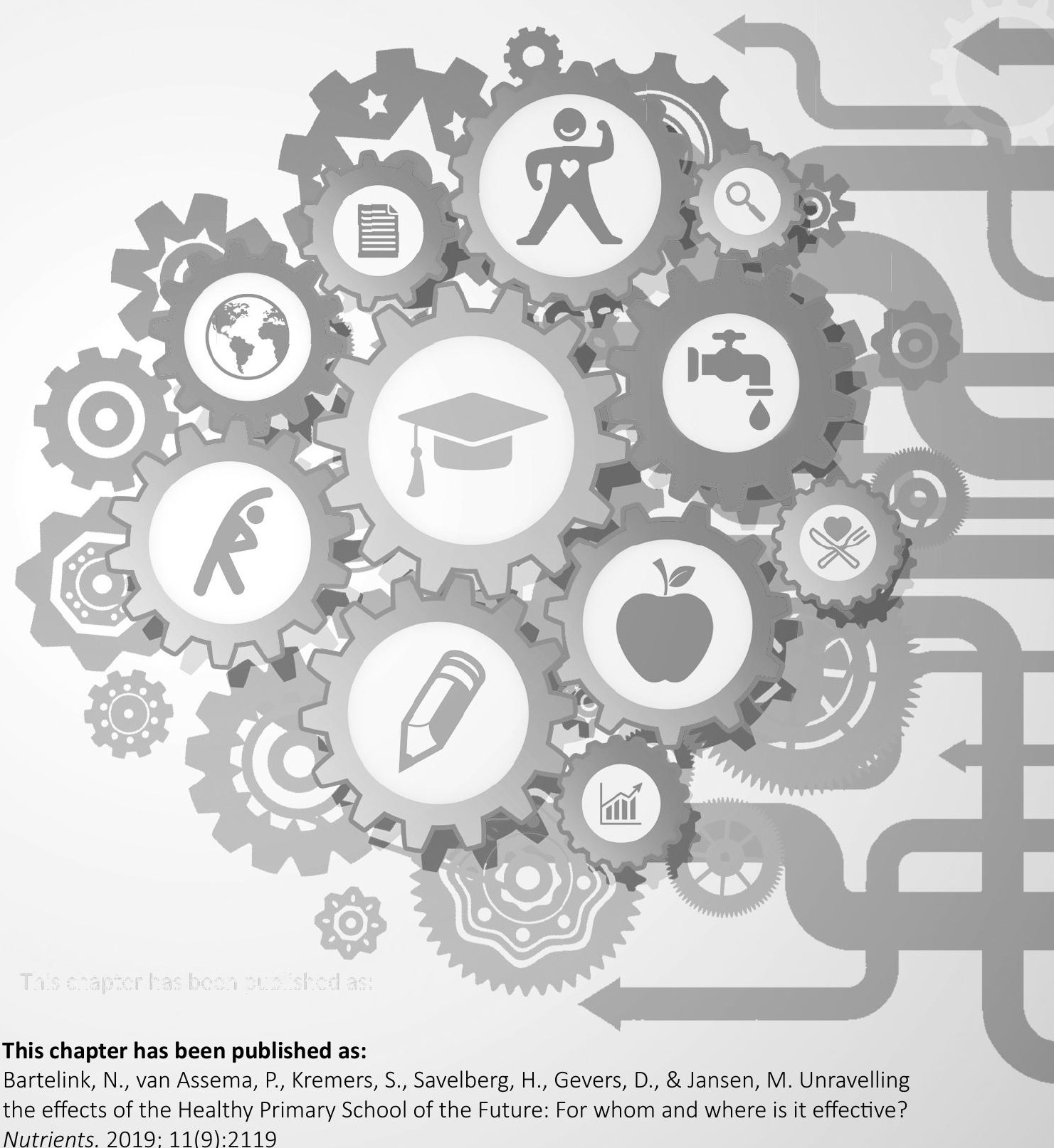
Nutrients. 2019; 11(9):2119 


\section{Abstract}

The 'Healthy Primary School of the Future' (HPSF) aims to integrate health and wellbeing within the whole school system. This study examined the two-year effects of HPSF on children's dietary and PA behaviours at school and at home and investigated whether child characteristics or the home context moderated these effects. The study ( $N=1676$ children) has a quasi-experimental design with four intervention schools, i.e., two full HPSF (focus: nutrition and PA) and two partial HPSF (focus: PA), and four control schools. Measurements consisted of accelerometry (Actigraph GT3X+) and questionnaires. Favourable effects on children's dietary and PA behaviours at school were found in the full HPSF; in the partial HPSF only on PA behaviours. Children in the full HPSF did not compensate at home for the improved health behaviours at school, while in the partial HPSF the children became less active at home. In both the full and partial HPSF, less favourable effects at school were found for younger children. At home, less favourable effects were found for children with a lower socioeconomic status. Overall, the effect of the full HPSF on children's dietary and PA behaviours was larger and more equally beneficial for all children than that of the partial HPSF. 


\section{Background}

Dietary and physical activity (PA) habits are formed at a young age [1], whereby unhealthy habits can already lead to overweight and obesity [2]. Schools can play an important role in promoting healthy behaviours in children, since a significant proportion of a child's day is spent there, and schools reach all children [14, 59, 86]. However, school is one of the microsystems, that interact to shape a child's health and well-being $[16,17]$. This means that the impact of changes in the school may also interact with the child's behaviour in other microsystems, e.g., the home context. This could lead to a transfer of improved health behaviours to the home context. However, compensatory behaviours might also occur: improvements in children's health behaviours at school (extra PA or healthier dietary behaviours) may be compensated at home by, e.g., a decrease in PA or unhealthier dietary behaviours [142, 145]. This school-home interaction might also cause different effects for children due to their home context. For example, a school-based intervention may be of greater benefit to those children with a high socioeconomic status (SES) background than to children with a low SES background, which leads to increased health inequities [120]. Moreover, parents can have different nutrition- and PA-related practices at home, e.g., parental behaviours and rules, which may also moderate the effects of school interventions on children's health behaviours [16, 17, 108, 146].

Additionally, not only might the home context moderate the effects of school health promotion efforts, child characteristics might also occur as effect modifier [16]. Several reviews have stated that even though the intention of school health promotion efforts is to reach all children, specific subgroups of children often benefit more than others. The review by Stewart-Brown et al. [47] found gender-specific results in several studies: some school-based interventions showed larger improvements on PA and dietary behaviours in girls and others in boys. Age-specific effects were also found, with some interventions being more effective in older children and others in younger children [47]. The review by Cook-Cotton et al. indicated that overweight children may respond more slowly or less well to school-based interventions than other children [108].

The 'Healthy Primary School of the Future' (HPSF) is a Dutch initiative that aims to improve the health and well-being of all children in the school by sustainably integrating health and well-being within the whole school system [55, 92]. The initiative is based on the principles of the Health Promoting School (HPS) framework, which includes a whole school approach, participation of teachers, children and parents, and partnerships in the local community [21]. HPSF is being investigated in an overall study among four intervention and four control schools by a multi-disciplinary research group [55, 92]. The overall study includes, among others, an extensive process evaluation and several effect evaluations. One of the effect evaluations found favourable intervention effects on children's health behaviours in the two intervention schools that focused on both healthy nutrition and PA [111]. The two intervention schools that focused only on PA 
found no effects, also not on children's PA behaviours. Since these results only presented overall effects, it is not known whether the effects occurred at school only or also at home. Nor is it known whether specific subgroups of children could be identified who benefitted more from HPSF in terms of PA and dietary behaviours [124].

The aim of the current study was to unravel the intervention effects of HPSF on children's health behaviours. Two main research questions were formulated: 1 ) What is the effect of HPSF on children's dietary and PA behaviours at school and at home? and 2) Did child characteristics or the home context moderate the effects of HPSF on children's dietary and PA behaviours?

\section{Methods}

\section{Study design}

The current study is part of the overall study that investigates HPSF [55] and uses a longitudinal quasi-experimental design with four intervention schools and four control schools. All eight participating schools are situated in the Parkstad region in the southern part of the Netherlands. This region has a low average SES, and unhealthy behaviours and overweight are higher in prevalence compared with the rest of the Netherlands [40]. Inclusion criteria for the schools were being a member of the educational board 'Movare', since they were one of the initiators of HPSF, and a minimum of 140 children in the study years two till five, to be able to study the effects of HPSF with enough statistical power. Ethical approval (14-N-142) for the overall study was given by the Medical Ethics Committee Zuyderland located in Heerlen (Parkstad, the Netherlands). A detailed description of the overall study and the recruitment of the eight schools is reported in Willeboordse et al. [55].

\section{The Healthy Primary School of the Future}

Three cooperating organizations, i.e., the regional educational board 'Movare', the regional public health services and Maastricht University, developed the HPSF initiative [55]. In line with the HPS framework [21], the initiative intends to establish a co-creation movement in schools for the development and implementation of health-promoting changes in different aspects of the school system, i.e., the school's physical and social environment, school's health policy, education, and school routines. In addition to the HPS framework, the aim was to create some form of positive disruption in the school by initiating two changes top-down: 1) a free healthy lunch each day and 2) daily structured PA and cultural sessions after lunch, both implemented by external pedagogical employees provided by childcare organizations. These changes are adapted bottom-up and should lead to momentum for more bottom-up processes to create additional health-promoting changes in school [92]. Two of the four intervention 
schools decided to implement both changes and are referred to as the 'full HPSF'. These schools also implemented additional health-promoting changes: they improved their health policy, e.g., policy regarding the consumption of water in school, they provided water bottles to all children, and have implemented an educational lunch once a week. The other two intervention schools only implemented the structured PA and cultural sessions and are referred to as the 'partial HPSF'. These schools did not implement any additional health-promoting changes. Each school selected one teacher as school coordinator, who managed HPSF in their school and all four schools involved teachers and parents in the adoption decision and the process of adapting the changes into the school context. Implementation started in all schools in November 2015. Overarching the four schools, the HPSF initiative was led by a project leader from Movare and an executive board with representatives of the three collaborating organizations, including the project leader. A project team was created with representatives of all partners involved: the four schools, Movare, regional Public Health Services, Maastricht University, the Limburg provincial authorities, childcare organizations, a caterer, and sports and leisure organizations.

\section{Study population}

All children (aged 4 to 12) and their parents in the eight schools ( $N=2326$ at T0) were invited to participate in this study. This included children from study years one to eight. Recruitment was done via information brochures for parents. The research team visited the classrooms to inform children about this study and encouraged them to ask their parents to participate [55]. Parents had to sign an informed consent form to participate in all measurements for themselves and their child(ren). The group of children included in this study were: at baseline (TO), children from study years one to seven; at T1, children from study years two to eight; and at T2, children from study years three to eight. Children who joined this study at T1 or T2 were included even though no baseline data were available. Children who switched to other schools between 2015 and 2017 were excluded.

\section{Measures}

Data were gathered annually during one week of measurements, conducted in September-November of 2015 (T0, previous to the start of HPSF in November 2015), 2016 (T1) and 2017 (T2). Inter-rater variability was minimized by training researchers according to a strict protocol. The data collection and data processing were identical to the evaluation of overall effects on children's dietary and PA behaviours [111].

\section{Potential effect modifiers}

Child characteristics: Children's study year and gender were collected via the database of the educational board Movare. Children's weight status was assessed by 
measurements of their height and weight. BMI was determined and age- and genderspecific BMI cut-off points were used to define children's weight status, i.e., nonoverweight versus overweight (including obesity) [115].

Children's socioeconomic background: A digital questionnaire for parents was used to obtain information about, among other things, children's SES. This was calculated as the mean of standardized scores on maternal education level, paternal educational level, and household income (adjusted for household size) [113]. The mean scores were categorized into low, middle and high SES scores based on tertiles.

Patterns of health-promoting parenting practices at home: The digital questionnaire for parents was also used to assess parents' nutrition-related practices ( $n=9)$ and PArelated practices $(n=14)$, e.g., modelling behaviour and encouragement. The questions were based on previous work by Gevers et al. [81, 98] and O'Connor et al. [80]. Each item described a practice by giving a statement, followed by some examples. Participants responded on a Likert scale from 1 (completely disagree) to 5 (completely agree). Two cluster analyses similar to Gevers et al. were conducted: one for the nutrition-related parenting practices and one for the PA-related parenting practices [81]. Detailed information of the results of each clustering is described in Additional file 1. Clustering of the nutrition-related parenting practices showed similar clusters of parents compared to the study of Gevers et al. [81]. The names of the clusters were: Cluster 1 ( $n=226$; 36.9\%) "High involvement and supportive"; Cluster 2 ( $n=102$; 16.7\%) "Low covert control and non-rewarding"; Cluster 3 ( $n=78 ; 12.7 \%$ ) "Low involvement and indulgent"; and Cluster 4 ( $n=206$; 33.7\%) "High covert control and rewarding". Clustering of the PA-related parenting practices also resulted in four clusters. The names of the clusters were: Cluster 1 ( $n=220 ; 35.0 \%)$ "High involvement and supportive"; Cluster 2 ( $n=133 ; 21.2 \%$ ) "Moderate involvement, indulgent of child's sedentary activities"; Cluster 3 ( $n=17 ; 2.7 \%$ ) "Low involvement and indulgent"; and Cluster 4 ( $n=258 ; 41.1 \%)$ "Moderate involvement, supportive of child's sedentary activities".

\section{Outcomes}

Children's PA levels - accelerometry: At the beginning of the measurement week all participating children from study years two to eight received an accelerometer for seven days (Actigraph $\mathrm{GT} 3 \mathrm{X}+, 30 \mathrm{~Hz}, 10$ s epoch). The monitor was attached to the hip with an elastic band and had to be worn all day except while sleeping or during activities in which water was involved (e.g., swimming, bathing and showering). The accelerometry data were processed using ActiLife version 6.13.3. Wear time validation was assessed using Choi's classification criteria [134]. Minimal wear time was defined as 480 min per day between 6 a.m. and 11 p.m. [135]. The first day of measurement was excluded to prevent reactivity [136]. Measurements containing at least three weekdays (after excluding the first measurement day) and one weekend day were used in the analyses [137]. The activity levels, classified using Evenson's cut-off points, were in 
counts-per-minute (CPM) [138]: sedentary behaviour (SB; $\leq 100$ CPM), light PA (LPA; 101 - 2295 CPM), and moderate-to vigorous PA (MVPA; $\geq 2296$ CPM).

Children's dietary behaviours - a parent-reported questionnaire: A digital questionnaire for parents was used to obtain information about their children's dietary behaviours. All parents of participating children (study years one to eight) received the questionnaire. Twelve questions from the Local and National Youth Health Monitor were used to assess children's dietary behaviours [131, 132]. Parents were asked about the number of days during the past week their child had breakfast, ate warm vegetables, salads or raw vegetables, fruits, consumed water and sugar-sweetened beverages (soft-, sports-, and energy drinks), and ate the following four snack types: chocolate, salted snacks, cookies, and soft ice-creams. A total score for healthy dietary behaviours (in mean days/week) was calculated by the mean number of days children consumed breakfast, fruits, vegetables (warm and cold), and water. A total score for unhealthy dietary behaviours (in mean days/week) was calculated by the mean number of days children consumed sugar-sweetened beverages and the four different snack types.

Children's dietary behaviours - two child-reported questionnaires: The first child questionnaire (for children of study years four to eight) was filled out by writing during class hours in the presence of at least one member of the research team. The questionnaire was used to assess, among other things, children's school water consumption ( 0 (almost) never - 1 sometimes (1-3 days per week) - 2 often (4-6 days per week) - 3 (every day)) and their breakfast intake. Breakfast questions consisted of recall questions (7 items) with yes/no answer options regarding the consumption of healthy food items, i.e., bread, cereals, butter, cheese, fruits, milk/yoghurt, and water, during breakfast that day. The items bread and cereals were combined into the food type grains, and milk/yoghurt and cheese were combined into the food type dairy. To give an indication about how healthy the children's breakfast was that day, the six different food types consumed were summed, and a dichotomous variable was created to study whether children consumed at least two of the food types during breakfast. The second questionnaire (for children of study years three to eight) was used to assess the children's lunch intake. The questions (except for an extra question regarding vegetable consumption during lunch) and the processing of the data were similar to those for the breakfast intake.

\section{Analyses}

Data were analyzed using IBM SPSS Statistics for Windows (version 23.0. Armonk, NY: IBM Corp). Missing data, including missing data at baseline, were imputed using a multiple imputation method with fully conditional specification (FCS) and 10 iterations, generating 50 complete datasets. Linear mixed-model analyses (continuous outcomes) and generalized estimating equations (binary outcomes) were used (see Bartelink et al. 
[111]) for the overall outcomes and school- and home-specific outcomes. Since measurements were repeated within participants, we used a two-level model with measurements as the first level and participants as the second level. The fixed part of the model consisted of group (full HPSF, partial HPSF, control), time (T0, T1, T2) and the interaction terms of group with time. We were not able to include class as a level in the model, because commonly more than one division of a class existed, e.g., $4 a$ or $4 b$, and children often did not have fixed class divisions for all years. To obtain children's settingspecific PA behaviours, the overall accelerometry data were divided by filters on wear time during school hours (school-specific PA) and wear time outside of school hours (home-specific PA). Regarding children's dietary behaviour, the two total scores (healthy and unhealthy dietary behaviours) were used as overall outcomes; children's lunch intake and their school water consumption as school-specific outcomes; and children's breakfast intake as home-specific outcomes. All analyses were adjusted for gender, study year at baseline, ethnicity, SES, and children's BMI z-score at baseline. A two-sided $p$-value $\leq 0.05$ was considered statistically significant. Standardized effect sizes (ES) were calculated, which were defined as estimated mean difference after two years divided by the square root of the residual variance at baseline (pooled over the intervention groups). Binary outcomes resulted in odds ratios (OR). To investigate whether the intervention effects were similar for all children, the following potential effect modifiers were considered: gender (boys/girls), study year at baseline (lower (14)/higher (5-8) grades), baseline weight status (non-overweight/overweight), SES (low/middle/high), and the patterns of nutrition-related parenting practices (four clusters) and PA-related parenting practices (four clusters). To assess potential effect modification, the interaction term group*time*effect modifier, with all corresponding two-way interactions, was added to the model [111]. When this interaction term was significant (here, we used a significance level of 0.10 to deal with the fact that the power of a test for interaction is relatively low [147] and we did not want to miss any effect modification), the intervention effects were reported for all categories of the effect-modifier separately.

\section{Results}

Of all children ( $n=2326$ ) invited to participate in the study, $60.3 \%$ joined the study at baseline ( $n=1403$ ). Because of the study's dynamic population, a total of 1974 children and their parents participated in the study within the two-year follow-up period (data collected at least at one time point). Due to the selection used for the current study, i.e., children were eligible for the current research only when they were in study years one to seven at baseline and did not switch schools, 1676 children were included in the analyses. See Additional file 2 for characteristics of the study sample. 


\section{Intervention effects}

Intervention effects at school

Both the full and partial HPSF resulted in significant favourable intervention effects on children's PA behaviours at school (Table 1). The time children spent in MVPA at school had increased significantly more in the full HPSF (ES=0.34) and partial HPSF $(E S=0.29)$ compared to the children in the control schools. Favourable trends were found for both sedentary time (full HPSF: ES=-0.20; partial HPSF ES=-0.17) and light PA at school (full HPSF: ES=0.11; partial HPSF ES=0.09). Regarding children's dietary behaviours at school, several favourable significant intervention effects were found in the full HPSF compared to the control schools: more children increased their water consumption at school (ES=1.14), and ate at least two food types during lunch $(\mathrm{OR}=2.98)$. In the partial HPSF, no significant intervention effects were found on children's dietary behaviours at school.

Intervention effects at home

Results showed no statistically significant favourable or adverse (compensatory) intervention effects of the full HPSF on children's PA and dietary behaviours at home (Table 1). In the partial HPSF, an adverse intervention effect was found at home for children's PA behaviours: the time children spent in light PA at home had decreased significantly more compared to children of the control schools. No significant favourable or adverse intervention effect was found for their dietary behaviours at home. 
Table 1. Intervention effects of HPSF at school and at home

\begin{tabular}{|c|c|c|c|c|c|c|}
\hline & \multicolumn{3}{|l|}{ Full HPSF vs. control } & \multicolumn{3}{|c|}{ Partial HPSF vs. control } \\
\hline & B (95\% C.I.) & $\mathrm{p}$ & ES & B (95\% C.I.) & $\mathrm{p}$ & ES \\
\hline \multicolumn{7}{|l|}{ Overall PA and dietary behaviours * } \\
\hline Sedentary (\% per day) & $-1.29(-2.39--.19)$ & .02 & -.23 & $-.17(-1.25-.90)$ & .76 & -.03 \\
\hline Light PA (\% per day) & $.94(.07-1.81)$ & .03 & .22 & $-.03(-.88-.82)$ & .95 & -.01 \\
\hline MVPA (\% per day) & $.36(-.10-.82)$ & .12 & .15 & $.19(-.26-.64)$ & .41 & .08 \\
\hline $\begin{array}{l}\text { Healthy dietary behaviours (mean } \\
\text { days/week) }\end{array}$ & $.19(.01-.37)$ & .04 & .19 & $-.02(-.20-.16)$ & .86 & -.02 \\
\hline $\begin{array}{l}\text { Unhealthy dietary behaviours } \\
\text { (mean days/week) }\end{array}$ & $-.07(-.21-.07)$ & .31 & -.11 & $.00(-.14-.15)$ & .96 & .01 \\
\hline \multicolumn{7}{|l|}{ PA and dietary behaviours at school } \\
\hline Sedentary (\% per day at school) & $-1.51(-2.96--.06)$ & .05 & -.20 & $-1.22(-.26-.19)$ & .10 & -.17 \\
\hline Light PA (\% per day at school) & $.70(-.51-1.92)$ & .29 & .11 & $.54(-.67-1.74)$ & .39 & .09 \\
\hline MVPA (\% per day at school) & $.76(.29-1.24)$ & $<.01$ & .34 & $.67(.22-1.12)$ & $<.01$ & .29 \\
\hline $\begin{array}{l}\text { Minimal two food types during } \\
\text { lunch** (\% yes) }\end{array}$ & $2.98(1.59-5.61)$ & $<.01$ & na & $.62(.36-1.05)$ & .08 & na \\
\hline School water consumption (0-3) & $1.17(.95-1.38)$ & $<.01$ & 1.14 & $-.02(-.23-.20)$ & .86 & -.02 \\
\hline \multicolumn{7}{|l|}{ PA and dietary behaviours at home } \\
\hline Sedentary (\% per day at home) & $-.47(-1.92-.99)$ & .53 & -.06 & $1.33(-.07-2.72)$ & .06 & .18 \\
\hline Light PA (\% per day at home) & $.33(-.74-1.41)$ & .55 & .06 & $-1.24(-2.27--.21)$ & .02 & -.23 \\
\hline MVPA (\% per day at home) & $.16(-.50-.82)$ & .63 & .05 & $-.08(-.71-.54)$ & .79 & -.02 \\
\hline $\begin{array}{l}\text { Minimal two food types during } \\
\text { breakfast** (\% yes) }\end{array}$ & $.95(.58-1.57)$ & .85 & na & $1.16(.71-1.89)$ & .54 & na \\
\hline
\end{tabular}

* For convenience purposes, this table also includes the results from the previously conducted study on overall effects of HPSF on children's PA and dietary behaviours [111]. The number of children was not always the same in the analyses due to differences in the methods of and response to the measurements. The number of children in each statistical test are reported in the previously conducted study.

** Binary outcome, B-value is presented as odds ratio.

Significance level: $p<0.05$.

Abbreviations: HPSF: Healthy Primary School of the Future; $\mathrm{Cl}$ : confidence interval; $\mathrm{p}$ : $\mathrm{p}$-value; ES: effect size; PA: physical activity; MVPA: moderate-to-vigorous physical activity; na: not applicable.

\section{Effect modifiers}

\section{Moderators of overall intervention effects}

Fewer moderators of intervention effects on children's dietary and PA behaviours were found in the full HPSF compared to the partial HPSF (1 vs. 4 significant moderators; Table 2). In the full HPSF, no moderators of children's dietary behaviours were found and one moderator of effects on children's PA behaviours was found. Gender moderated the effect on MVPA, as boys had increased their time in MVPA significantly more compared to boys in the control schools ( $E S=0.34$ ); this effect was not found in girls (ES=0.02). In the partial HPSF, study year and SES were found to be significant 
moderators. Study year moderated the intervention effects on the time children spent sedentary and in light PA, with a favourable trend found in older children and an adverse trend in younger children. SES moderated the effects on the time spent in MVPA and unhealthy dietary behaviours. Each SES tertile showed a different trend, with a lack of consistency. In both the full and partial HPSF, no moderation was found by children's weight status and parenting practices.

\section{Moderators of intervention effects at school}

Fewer moderators of intervention effects at school were found in the full HPSF compared to the partial HPSF (2 vs. 4 significant moderators; Table 3). In both the full and partial HPSF, intervention effects at school were moderated by study year. In the full HPSF, study year moderated the effects on sedentary time and light PA. In the partial HPSF, study year moderated the effect on sedentary time, light PA and school water consumption. Consistently throughout the subgroup analyses, it was found that in older children the effects were more favourable than in younger children. No other moderations were found in the full HPSF. In the partial HPSF, school water consumption was significantly moderated by gender. An adverse significant intervention effect was found for girls $(E S=-0.36)$, and a trend in a favourable direction was found for boys (ES=0.26).

\section{Moderators of intervention effects at home}

Fewer moderators of intervention effects at home were found in the full HPSF than in the partial HPSF (3 vs. 5 significant moderators; Table 4). In both the full and partial HPSF, SES moderated the intervention effects on children's PA behaviours at home. In general, adverse effects or trends at home were found for the children in the lowest SES tertile and favourable effects or trends for the children in the highest SES tertile. In the full HPSF, a significant favourable effect on the time spent sedentary (ES=-0.34) was found for the children in the highest SES tertile. In the partial HPSF, a significant adverse effect for sedentary time (ES=0.46) and light PA (-0.52) was found for the children in the lowest SES tertile. Additional file 3 presents all pairwise comparisons. The comparison between the lowest and highest SES tertile consistently shows a different effect at home compared to the effects at school. The results showed consistent differences in effect between the lowest and highest SES tertiles regarding PA behaviours at home ( $B>1$ in 5 out of 6 comparisons, Table S2c), whereas no differences in effect were found at school ( $\mathrm{B}<1$ in all six comparisons, Table $\mathrm{S} 2 \mathrm{~b}$ ). As an exception, this pattern was not found for children's breakfast intake. No other significant moderations were found in the full HPSF for children's PA and dietary behaviours at home. In the partial HPSF, the intervention effects at home on sedentary time, light PA, and on the consumption of at least two food types during breakfast were also moderated by weight status. Favourable trends were found at home in overweight children and adverse trends or effects were found in non-overweight children. 


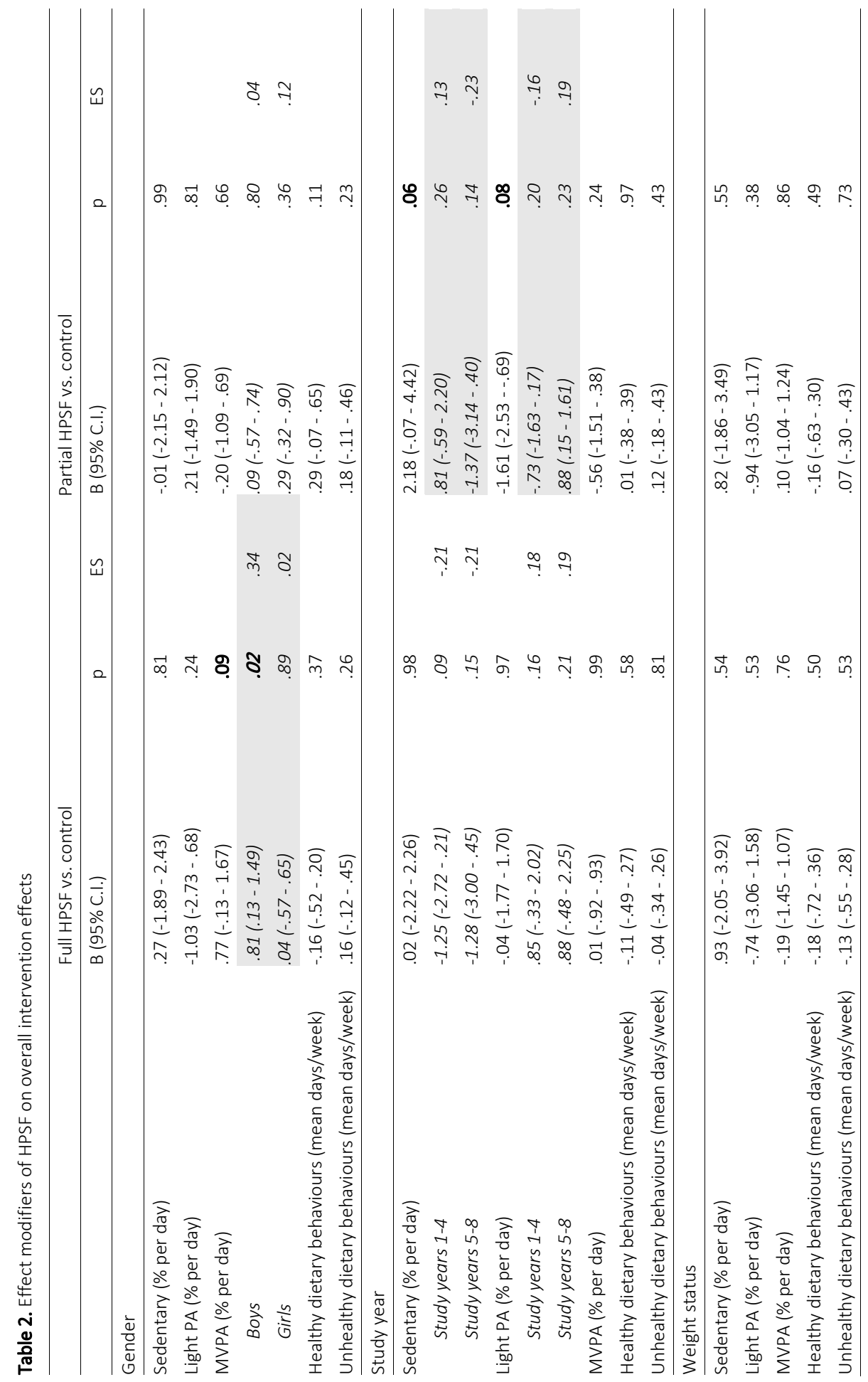


For whom and where is HPSF effective?

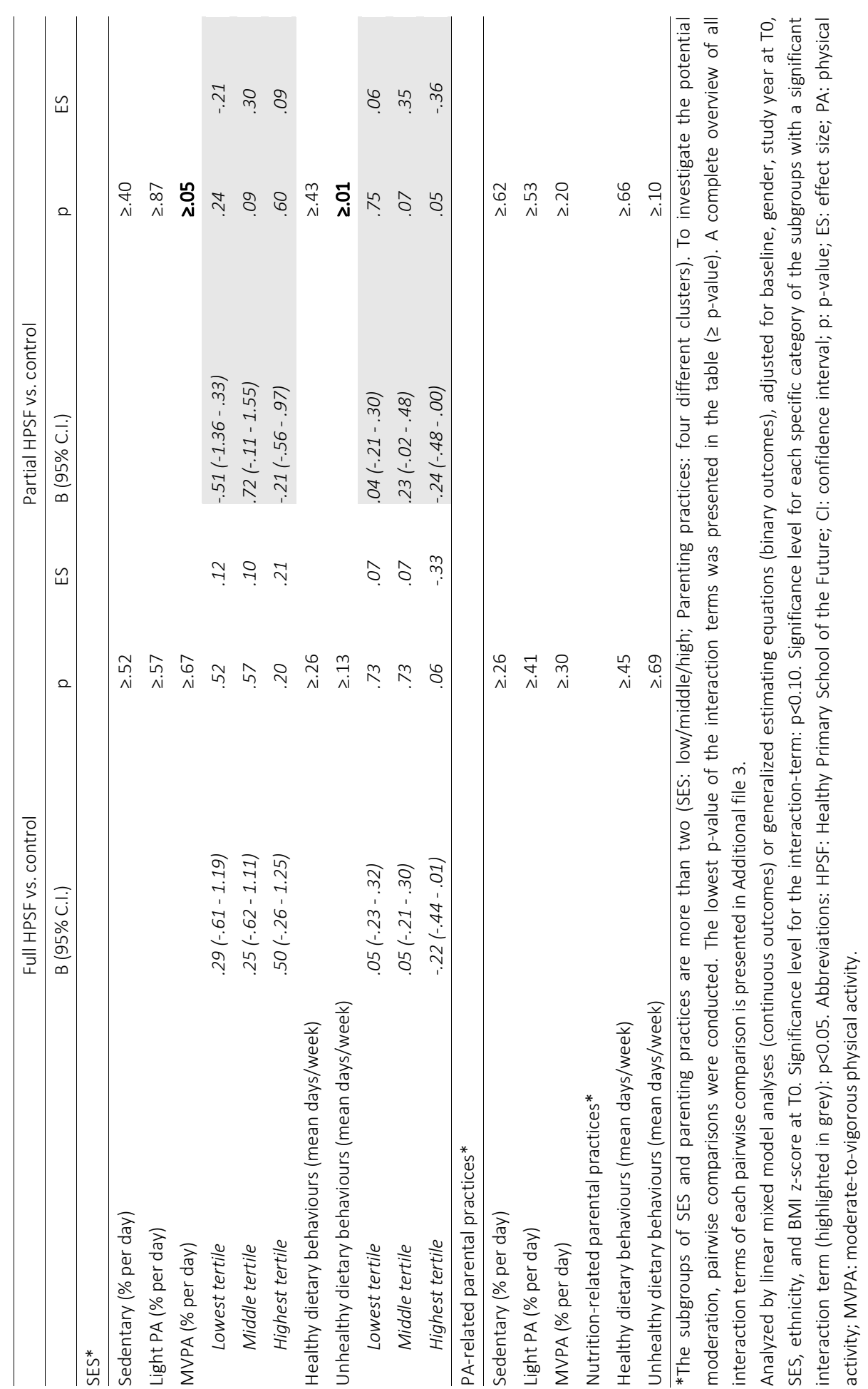




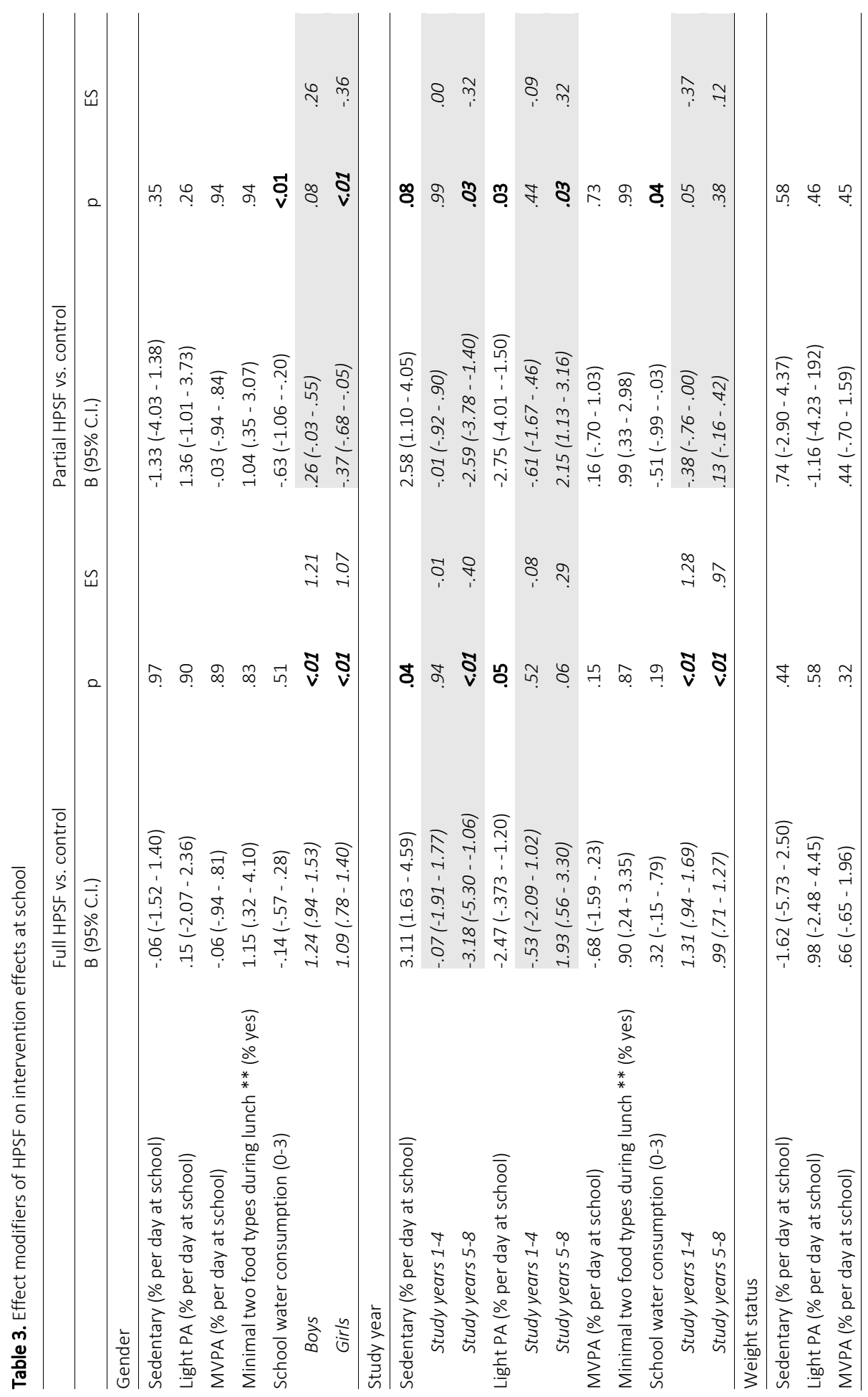




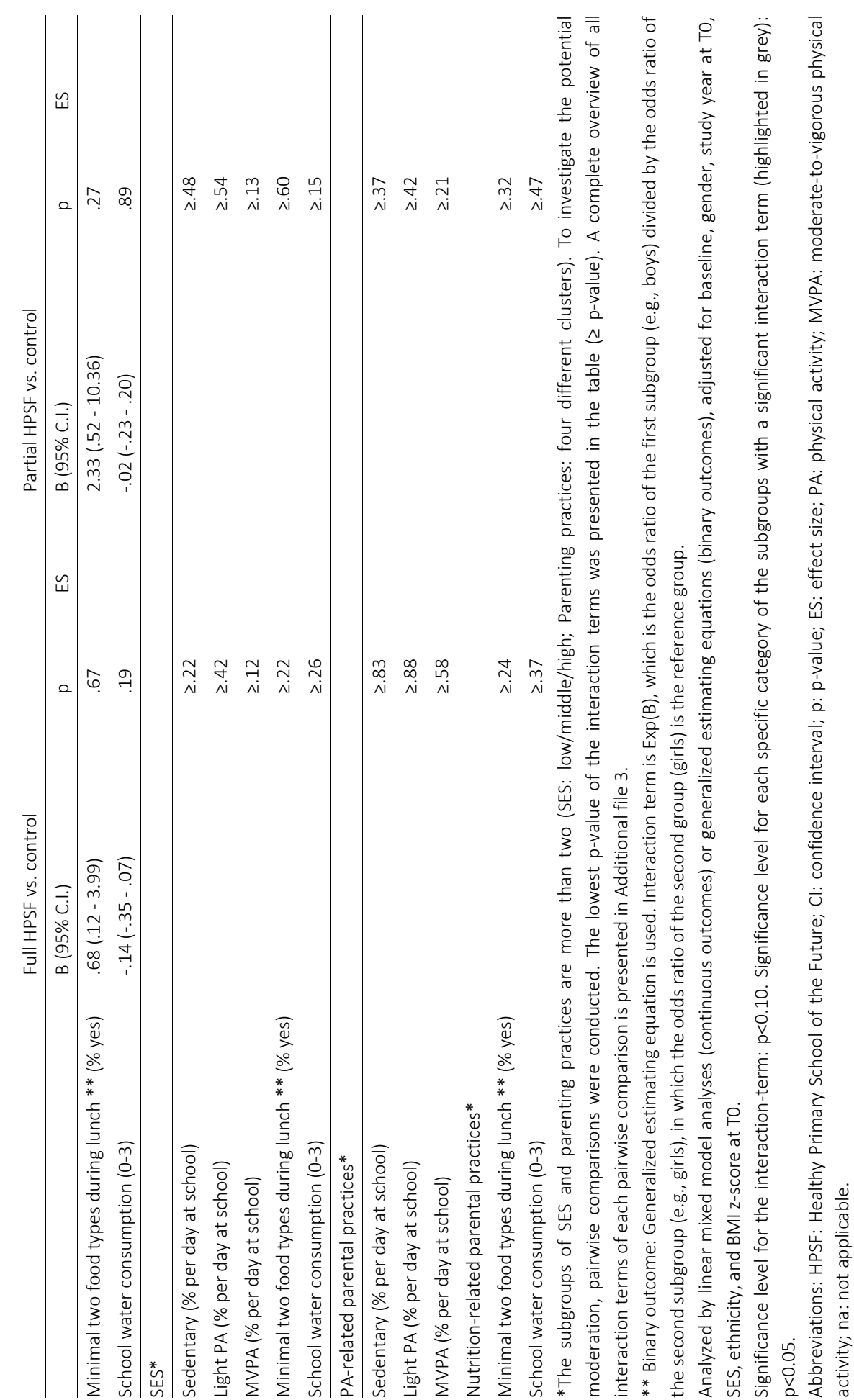




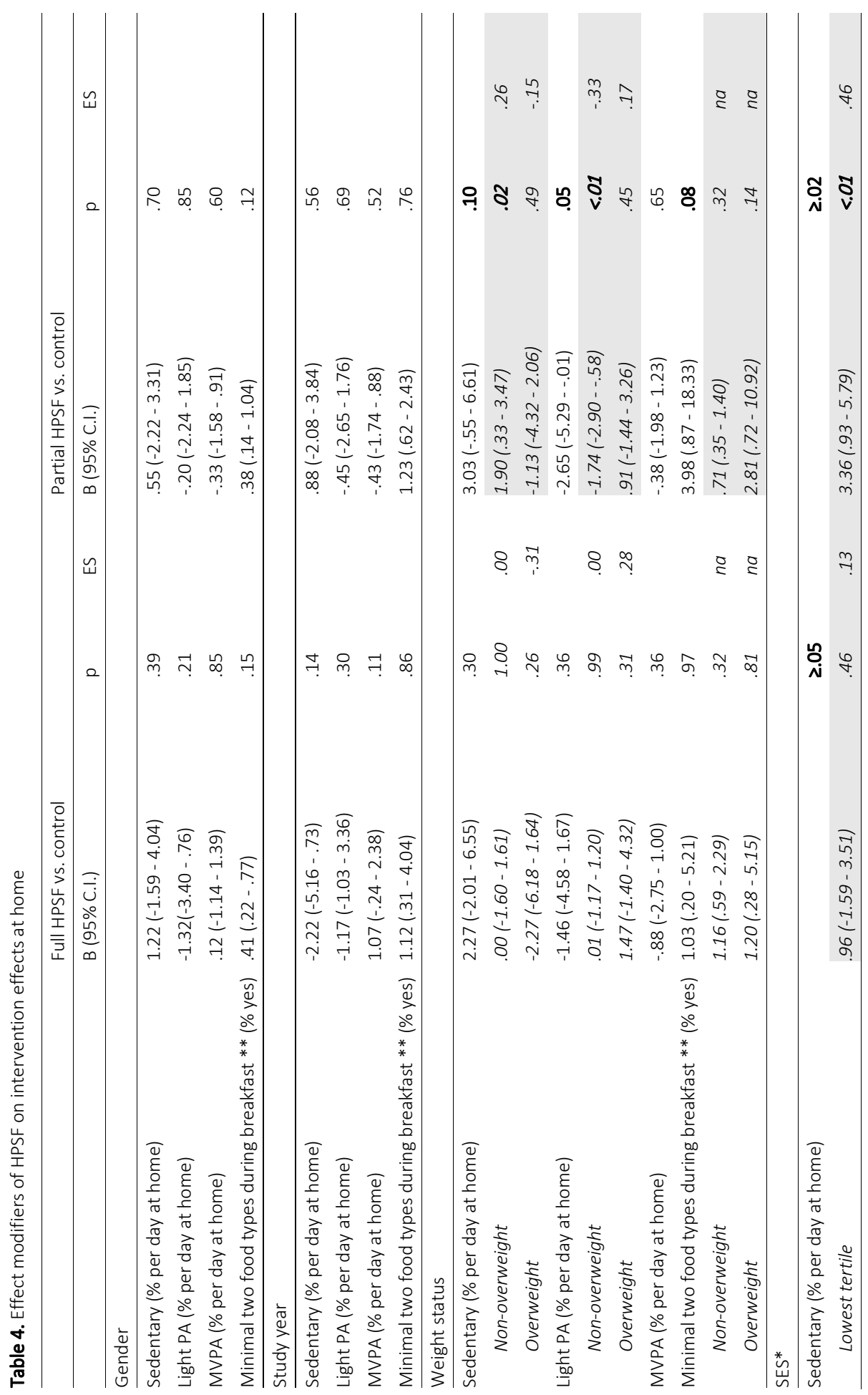




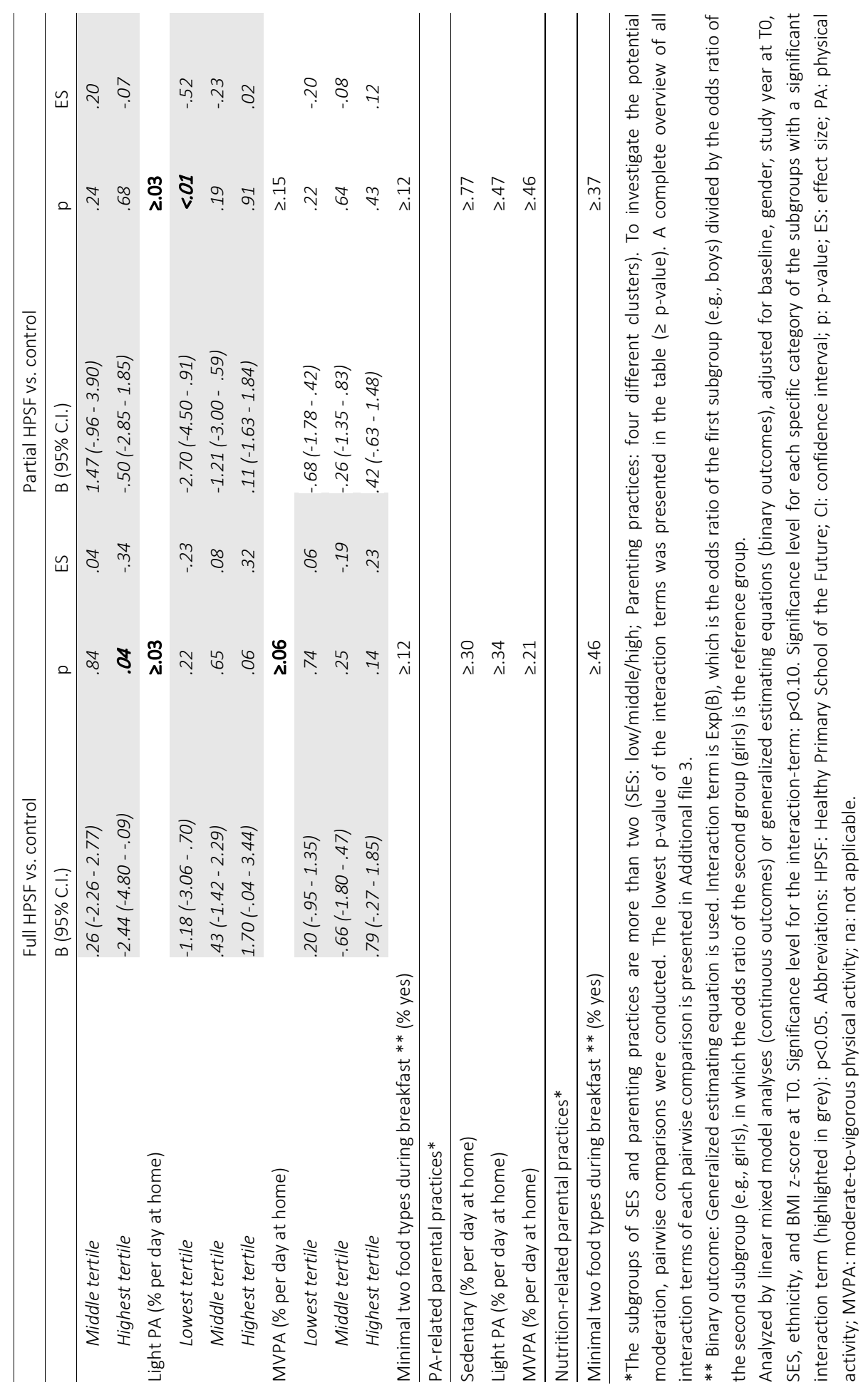




\section{Discussion}

The current study aimed to unravel the effects of HPSF on children's dietary and PA behaviours. It investigated the intervention effects of HPSF at school and at home. Results showed that the time children spent in MVPA at school had increased in both the full and partial HPSF. However, children of the full HPSF did not compensate at home for the improved health behaviours at school, while in the partial HPSF, the results indicated that the children did compensate by becoming less active at home. Children's dietary behaviours at school improved in the full HPSF, without compensating for these improvements at home.

The current study also investigated whether child characteristics or the home context moderated the intervention effects of HPSF. The results showed that the effects in the partial HPSF were influenced more often by moderators (in total, 13 significant moderators $(18.6 \%)$ ) than in the full HPSF (in total, six significant moderators $(8.6 \%)$ ), which indicates that the full HPSF had a more equal beneficial effect for all children. The findings indicated that the intervention effects of HPSF on children's PA and dietary behaviours were mainly moderated by SES and study year. This is in contrast to a previous study on the effects of HPSF on children BMI z-scores, in which no moderators emerged [124]. An explanation for the contrasting results may be that the effect on children's BMI z-score is a result of the co-existence and interaction of the children's nutrition and PA behaviours [118]: a moderating impact on one health behaviour may therefore not automatically lead to moderation of intervention effects on children's BMI z-score.

The effects on children's PA behaviours at school were moderated by study year in both the full and partial HPSF: older children benefitted consistently more from HPSF than younger children. This may indicate that the activities in school were more appropriate for the older children. This is in line with the results and conclusions of several studies that indicated that children of different ages have different needs regarding PA-activities $[16,148]$. Previous research has for example shown that older children's activity levels were more negatively affected by the number of peers present, while younger children were more negatively affected by the number of supervisors [149]. Therefore, it is recommended when implementing PA-related activities to ensure that either they are appropriate for all children or that age-specific PA-activities are implemented.

The findings in this study showed that HPSF succeeded in creating equal effects on children's PA and dietary behaviours at school, independent of the children's background (SES, parenting practices). However, the findings also showed that the children's socioeconomic background did influence the effects at home. The children with the lowest SES scores did not improve their PA behaviours at home; results even showed compensating behaviours of PA in these children at home. For the children from the highest SES group in the full HPSF, however, a transfer of the effects on PA 
was found from school to the home context. This suggests that the changes in the full HPSF schools have led to such an impact that these children also engaged in more PA after school. In contrast, these favourable effects at home did not occur in the children with the lowest SES scores. The compensation of PA at home in the lowest SES group has led to opposite effects at school and at home for these children: at school, their PA behaviours became more favourable; at home, they became less favourable. Since these opposite effects were especially found for the children with lowest SES scores, it may contribute to an increased socioeconomic health equity gap. In addition, the partial HPSF also showed a moderating effect of SES on children's overall dietary behaviours, with less favourable, and even adverse effects for the children with lower SES scores. Children in these schools, in contrast to children in the full HPSF, brought all foods and drinks that they consumed at school from home. This means that the dietary behaviours that are included in this outcome are actually dietary behaviours that have their origin in the home context. The moderating effect of SES on this outcome in the partial HPSF seems to indicate that not only children's PA behaviours at home, but also their dietary behaviours at home are moderated by SES, with consistently less favourable effects for children with lower SES scores. It should be noted though that since all schools are located in a low SES area, the SES tertiles used in this study are relative and not absolute scores. This means that the average SES score of children in this study sample is low compared to the average of the Netherlands [121]. Nevertheless, the differences in effect at home among children with lower and higher socioeconomic background demonstrate the interaction between two main microsystems of a child, i.e., school and home. This underlines that the school is an open system and interacts with other microsystems, such as home or the neighbourhood, to shape a child's health and wellbeing $[16,17]$. According to a study by Gubbels et al., larger consistency across microsystems leads to more favourable effects on children's health behaviours [150]. Therefore, it can be recommended for school health promotion efforts to include the home context in the HP changes, e.g., homework assignments that include parents, and/or to focus directly on health promotion in the home context [151]. In this way, the child's environment enables healthier choices both at school and at home. This creates more consistency between the different microsystems of a child, particularly for children with lower socioeconomic backgrounds.

No moderating effect of parenting practices was found in the current study, which indicates that the effects of HPSF were not strengthened or weakened by parenting practices at home. This is in line with the results of a previous Dutch study conducted in secondary schools, which investigated whether family environmental factors affected changes in adolescent's dietary behaviour who participated in a school health promotion program [152]. These findings indicate that it is not so much the parenting practices that explain the differences in home effects across SES groups, but that other aspects in the home context, such as the physical environment in the neighbourhood, may explain these differential intervention effects at home. 


\section{Strengths and limitations}

The quasi-experimental design can be seen as a limitation of the study, since we were unable to (cluster-) randomize schools. To deal with this limitation, we controlled for BMI z-score at TO, gender, study year at TO, SES score, and ethnicity in all analyses. However, despite the lack of randomization, the design enabled us to test the effectiveness in terms of differences in children's health behaviours between the three school groups over time, and we were able to enrol schools on the basis of motivation, which reflects the real-life situation of school health promotion. Another limitation is the multiple statistical testing in this study, which may increase the likelihood that the observed statistical differences have arisen by chance. Methodological strengths of the study include the objectively measured PA levels, all collected in the same season, and the matching of all measurements in the same week to prevent overburdening of the people in the school. Furthermore, since we had insight into the specific school hours of all included schools, we were able to separate children's PA behaviours at school and at home. Another strength of the study is that child-reported data were collected regarding both their breakfast and lunch. This created the possibility to investigate the effects of HPSF on specific dietary behaviours of children at school and at home. It should be mentioned though that by categorizing the consumption of breakfast as a dietary behaviour at home, the assumption was made that children consumed their breakfast at home. Even though this is very common in the Netherlands, we do not have the data to confirm this assumption. Moreover, to investigate as much as possible children's actual dietary behaviours during these meals, the answers of children without interference of parents were used. The specific effects of HPSF on both meals became more visible due to the use of comparable questions. In general, the assessment of children's dietary behaviours had its limitations as only questionnaires were used, which are subjective measurements and may lead to socially desirable answers [143]. To assess children's dietary behaviours more objectively, future research could include image collection methods. The parenting practices questionnaire, which also had these subjectivity limitations, was based on a validated questionnaire by Gevers et al. [98]. However, we were not able to include all practices described by Gevers et al., due to limitations in the length of the questionnaire as many other aspects were included in this questionnaire. The reduction in assessed practices was based on expert judgement; practices were only deleted when they were more or less similar to another practice. Due to this systematic approach we were still able to conduct a cluster analysis, which led to the same four clusters [81]. The findings of this cluster analysis seem to indicate that also in another study sample the four patterns are visible, which is a next step in the validation of these patterns of parenting practices. Future research is needed to validate these findings and to investigate whether these clusters are also applicable in other study samples. 


\section{Conclusion}

The effect of the full HPSF on children's dietary and PA behaviours was not only larger, but also more equally beneficial for all children than that of the partial HPSF. In both the full and partial HPSF, less favourable effects at school were found for younger children. At home, less favourable effects were found for children with lower SES scores. It is recommended to include the home and neighbourhood context in health promotion efforts in order to create more consistency across the different microsystems of a child. This may particularly benefit children from lower socioeconomic backgrounds. 


\section{Appendix Chapter 6}

\section{Additional file 1 . Clustering of parenting practices}

Clustering of nutrition-related practices of parents

Preparation of the data

Before running cluster analyses, parents with missing data on the practices were excluded $(n=0)$, univariate outliers were replaced by the mean score plus three standard deviations ( $0.83 \%$ of all responses on FPPs), and multivariate outliers (15 cases) were eliminated from the further analyses.

The four clusters

Cluster 1 ( $\mathrm{n}=226 ; 36.9 \%$ ), labelled "high involvement and supportive", was characterized by relatively high scores on most types of nutrition-related parenting practices.

Cluster 2 ( $n=102 ; 16.7 \%$ ), labelled "low covert control and non-rewarding", consisted of parents with relatively moderate scores on most practices, but with lower scores on accessibility of healthy foods and low use of emotional feeding.

Cluster 3 ( $n=78 ; 12.7 \%)$, labelled "low involvement and indulgent", had relatively low scores on most types of nutrition-related parenting practices.

Cluster 4 ( $\mathrm{n}=206 ; 33.7 \%)$, labelled "high covert control and rewarding", was characterized by relatively moderate scores on most practices, but with higher scores on accessibility of healthy foods and high use of emotional feeding. 


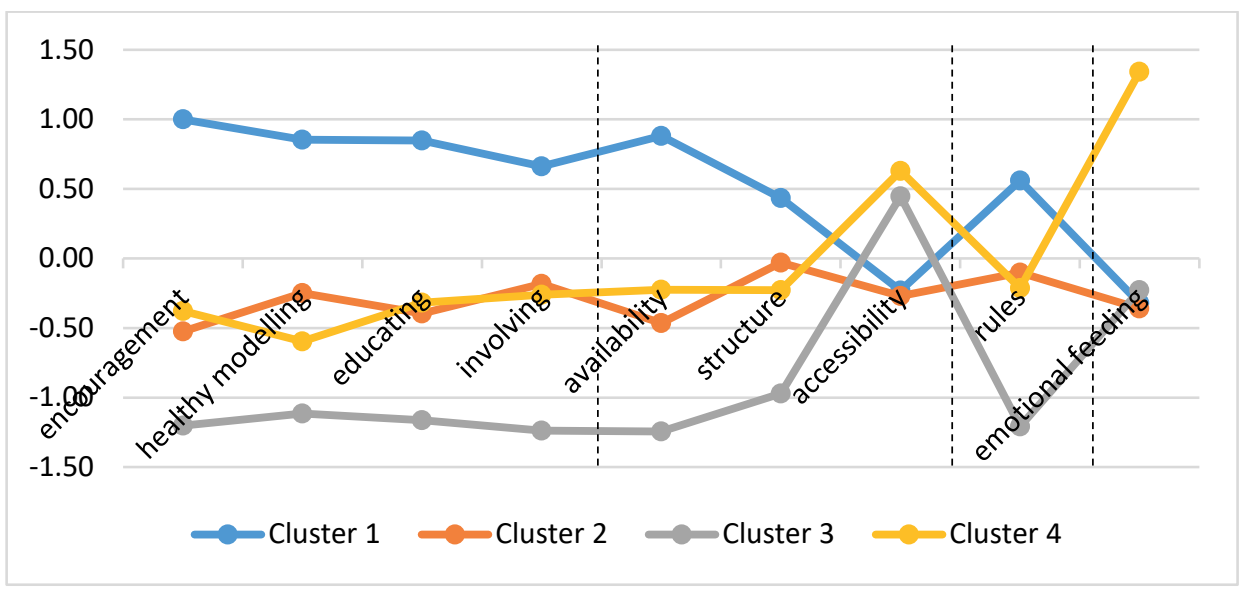

Figure S1. Graphical view of the four-cluster solution based on mean z-scores for all nutrition-related parenting practices $(n=612)$

Higher scores indicate more frequent use of the FPP; Each of the successive graphical areas represents a distinct category of nutrition-related parenting practices, i.e., responsiveness, structure, behavioural control and psychological control; Cluster 1 "high involvement and supportive": blue line; Cluster 2 "low covert control and non-rewarding": orange line; Cluster 3 "low involvement and indulgent": grey line; Cluster 4 "high covert control and rewarding": yellow line.

\section{Clustering of PA-related practices of parents}

Preparation of the data

Before running cluster analyses, parents with missing data on the practices were excluded $(n=5)$, univariate outliers were replaced by the mean score plus three standard deviations ( $0.68 \%$ of all responses on FPPs), and multivariate outliers (16 cases) were eliminated from the further analyses.

\section{Exploration of the most optimal clustering}

Since the clustering of Gevers et al. [81] focused only on nutrition-related parenting practices, we conducted the full exploration of the most optimal clustering. Ward's method indicated that a four-cluster solution gave the best fit, in view of the change in agglomeration coefficients. After considering this outcome and the dendrogram, we conducted k-means cluster analyses using 3- and 4-cluster solutions, from which a fourcluster solution was derived. After replicating the full two-step clustering approach in two subsamples, we obtained a Cohen's kappa of 0.814 , indicating substantial stability of the cluster solution. 


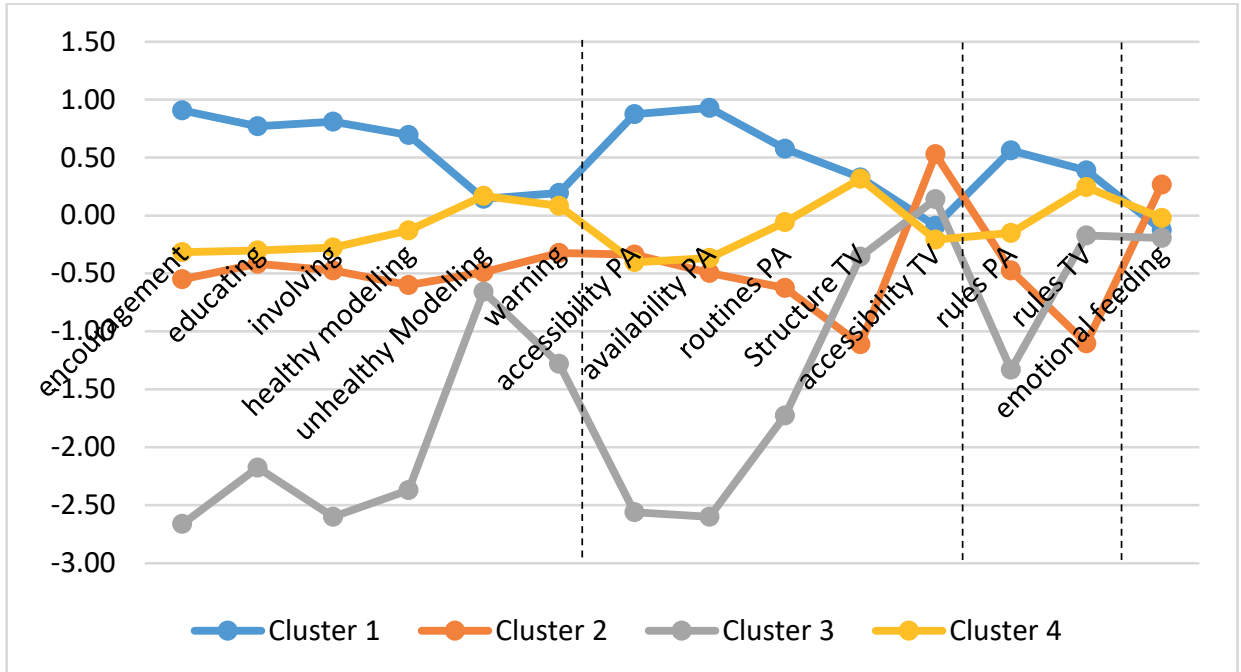

Figure S2. Graphical view of the four-cluster solution based on mean z-scores for all PA-related parenting practices $(\mathrm{n}=628)$

Higher scores indicate more frequent use of the PA-PP; Each of the successive graphical areas represents a distinct category of PA-related parenting practices, i.e., responsiveness, structure, behavioural control and psychological control. Cluster 1 "high involvement and supportive": blue line; Cluster 2 "moderate involvement, indulgent of child's sedentary activities": orange line; Cluster 3 "low involvement and indulgent": grey line; Cluster 4 "moderate involvement, supportive of child's sedentary activities": yellow line.

The four clusters

Cluster 1 ( $\mathrm{n}=220 ; 35.0 \%$ ), labelled "high involvement and supportive", was characterized by relatively high scores on most types of PA-related parenting practices.

Cluster 2 ( $n=133 ; 21.2 \%$ ), labelled "moderate involvement, indulgent of child's sedentary activities", was characterized by relatively moderate scores on most practices, but with lower scores on sedentary-related practices.

Cluster 3 ( $n=17 ; 2.7 \%$ ), labelled "low involvement and indulgent", had relatively low scores on most types of PA-related parenting practices.

Cluster 4 ( $\mathrm{n}=258 ; 41.1 \%)$, labelled "moderate involvement, supportive of child's sedentary activities", was characterized by relatively moderate scores on most practices, but with higher scores on sedentary-related practices. 


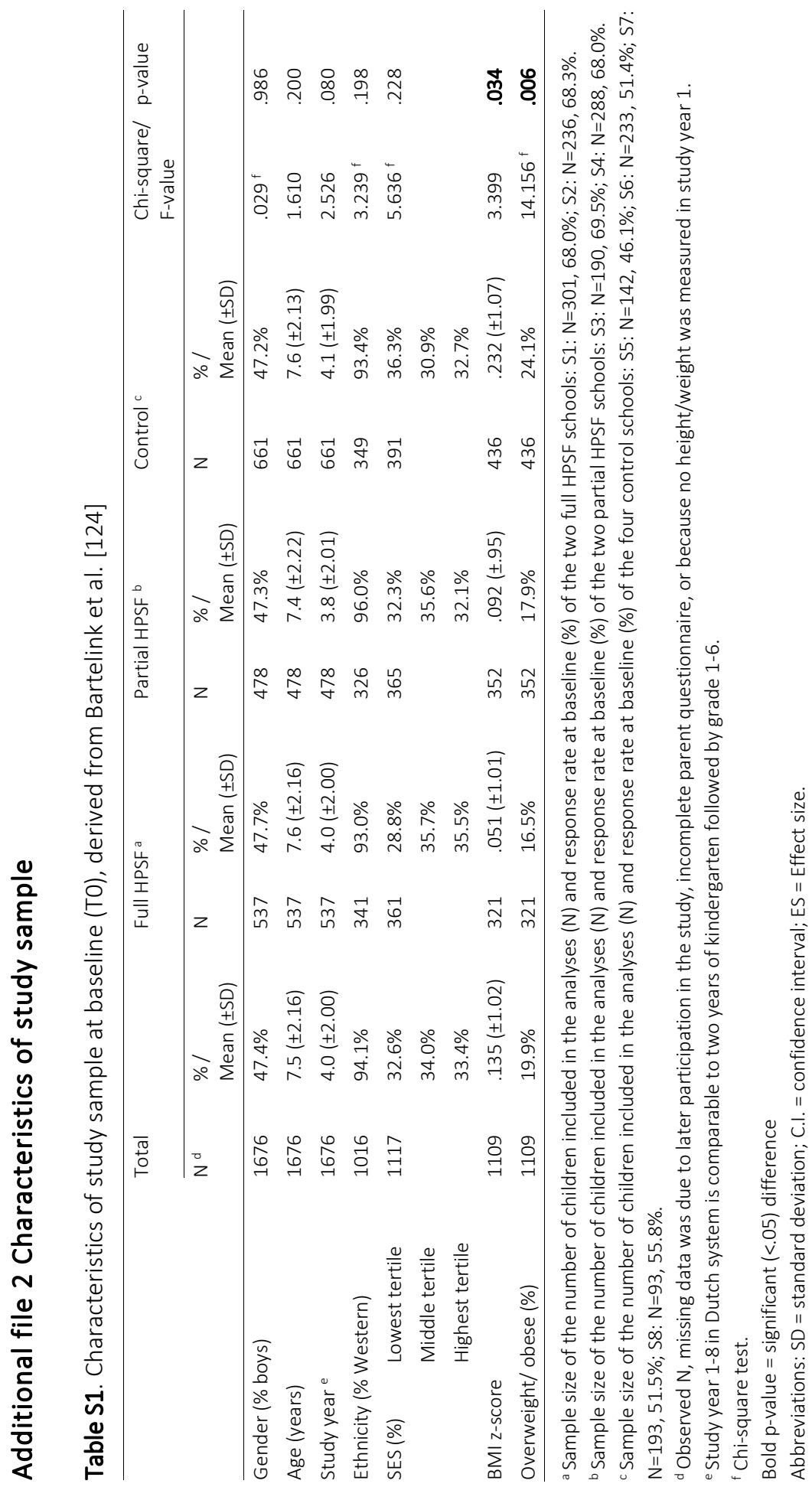




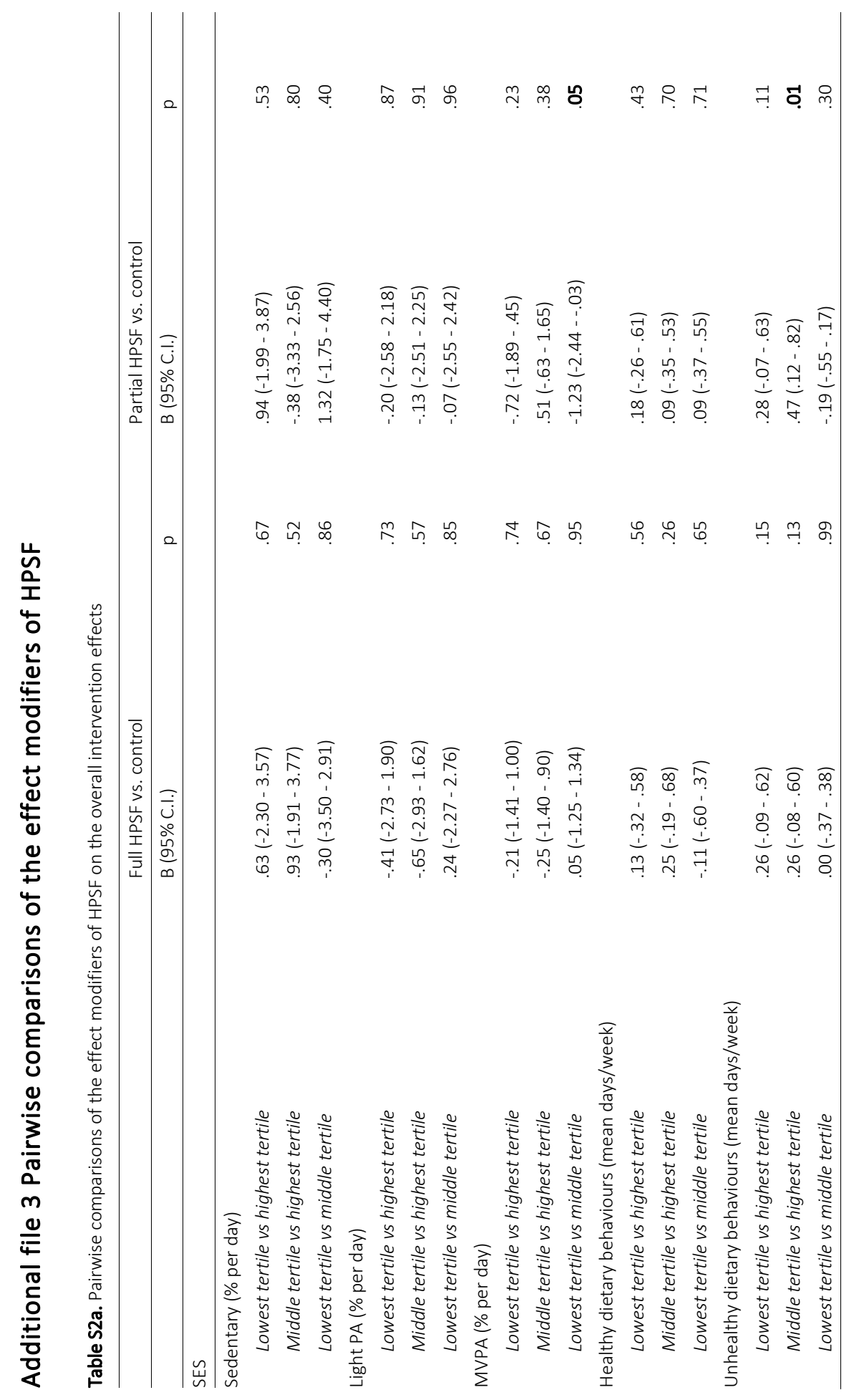


For whom and where is HPSF effective?

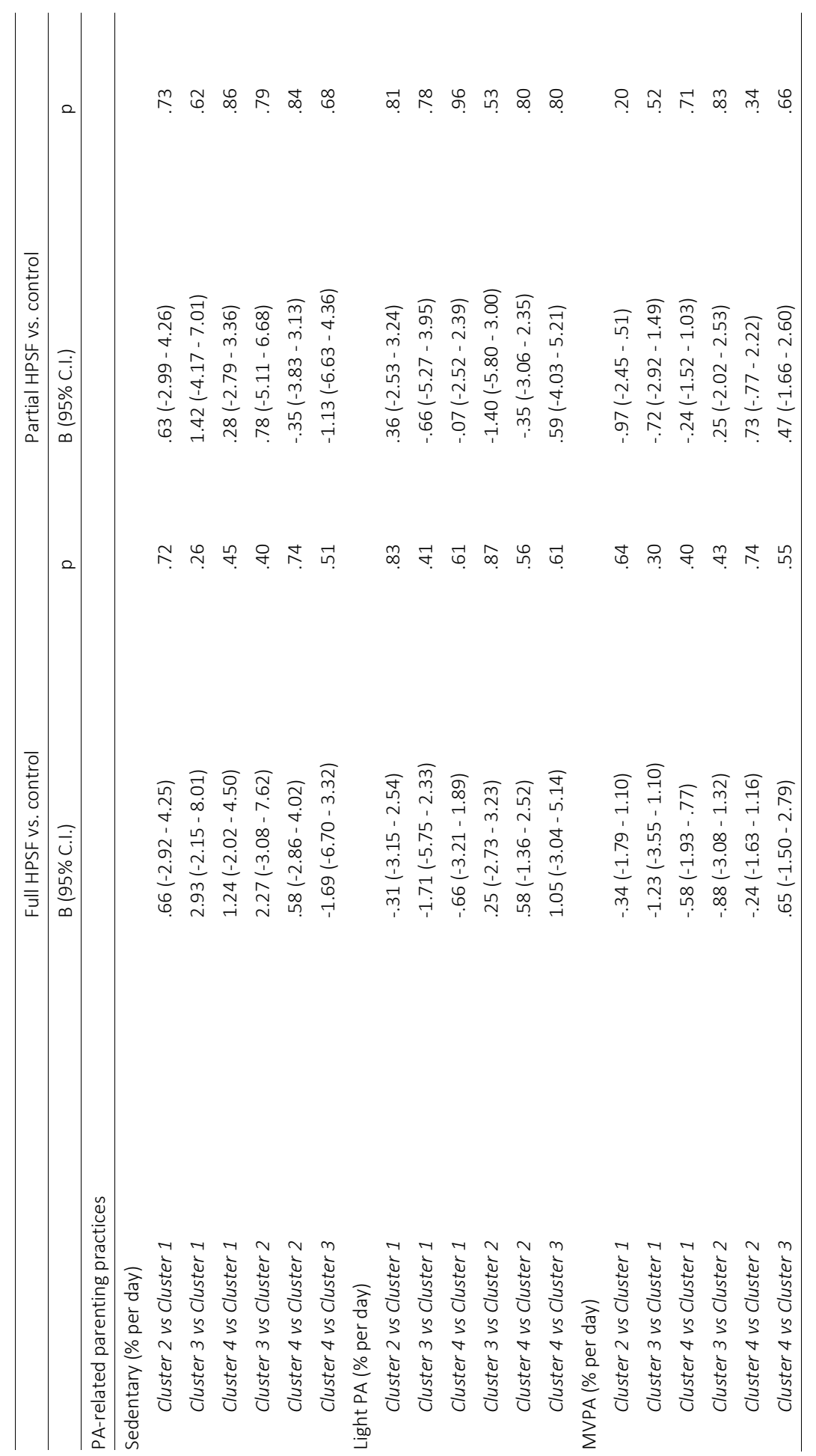




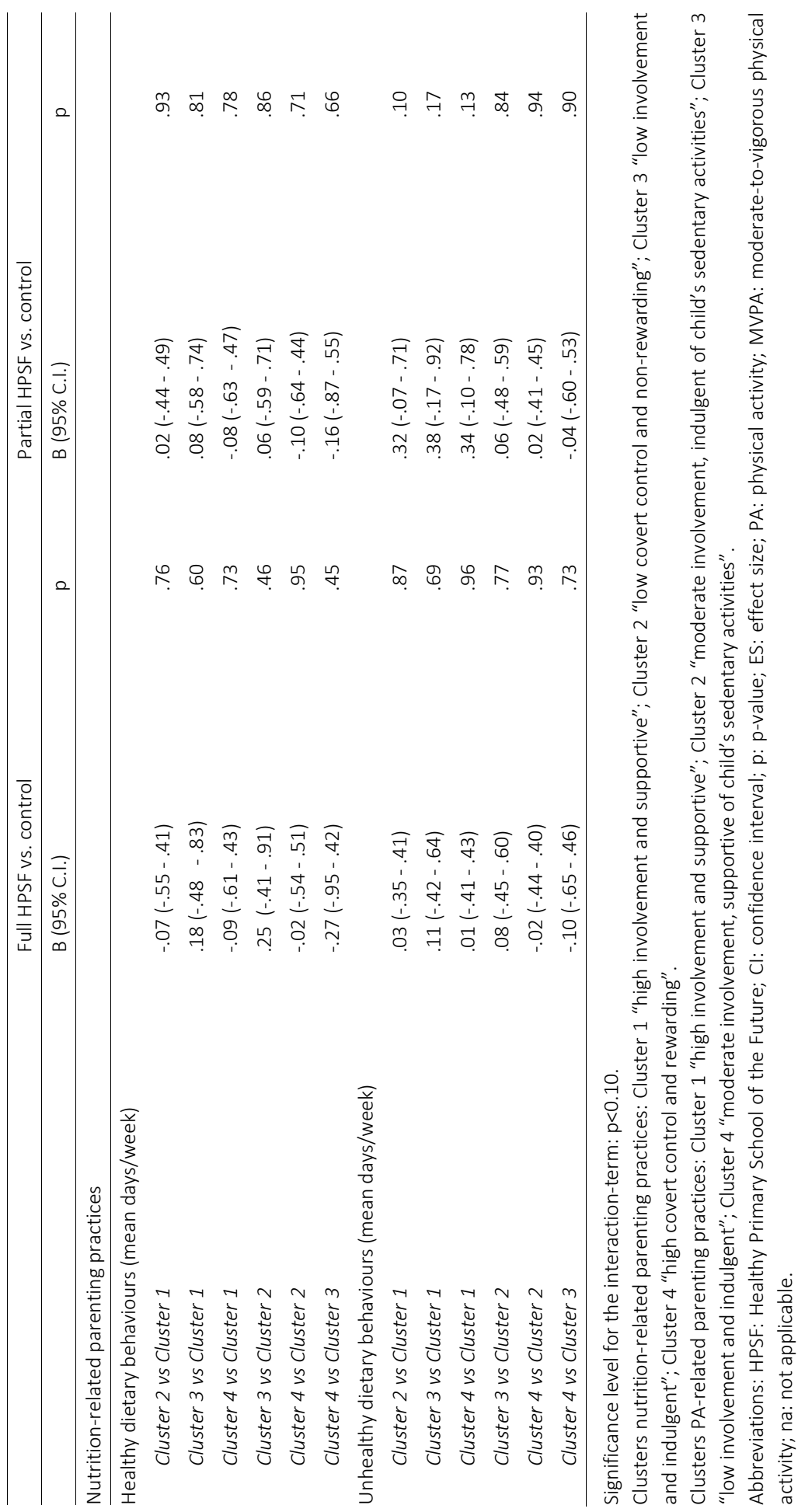


For whom and where is HPSF effective?

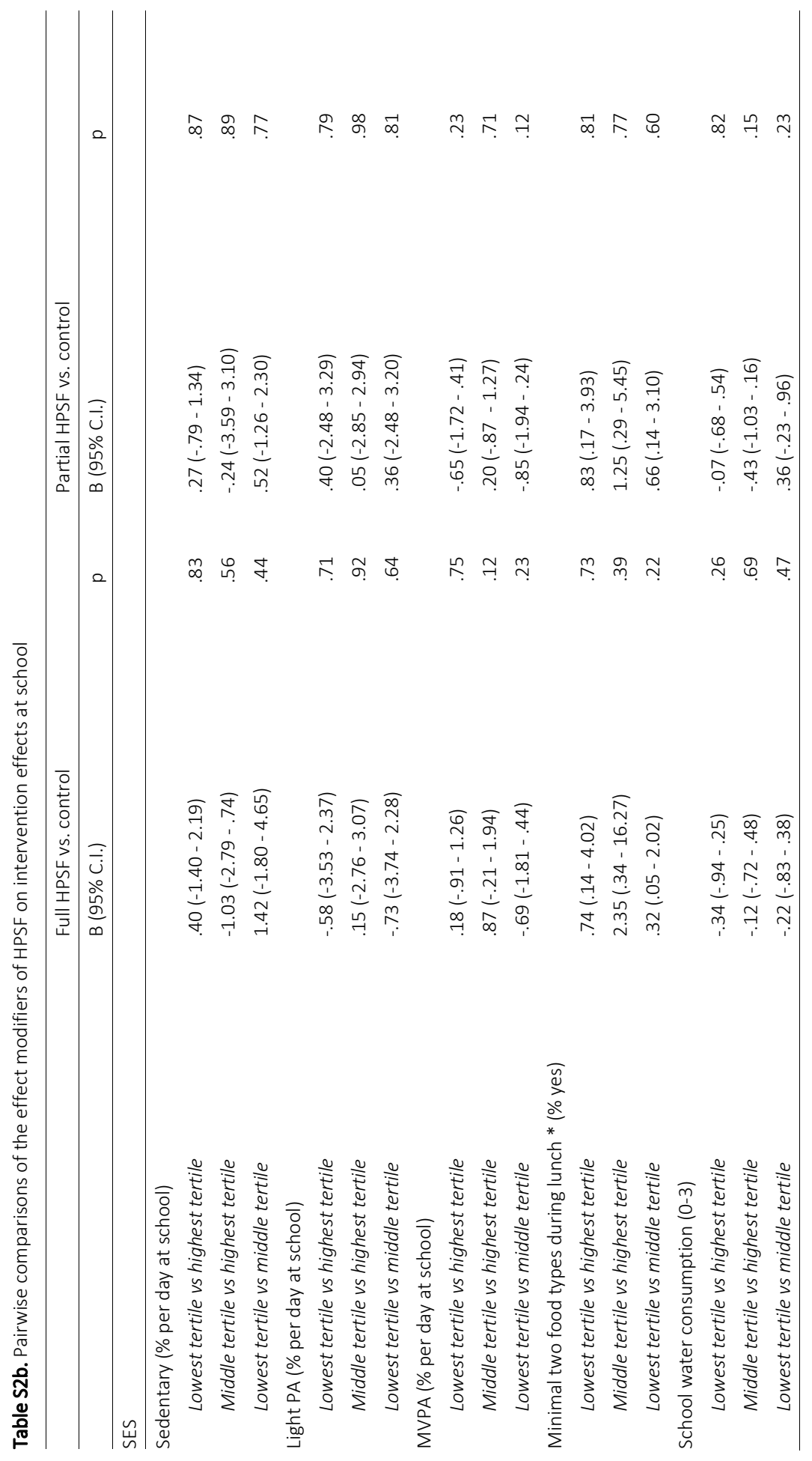




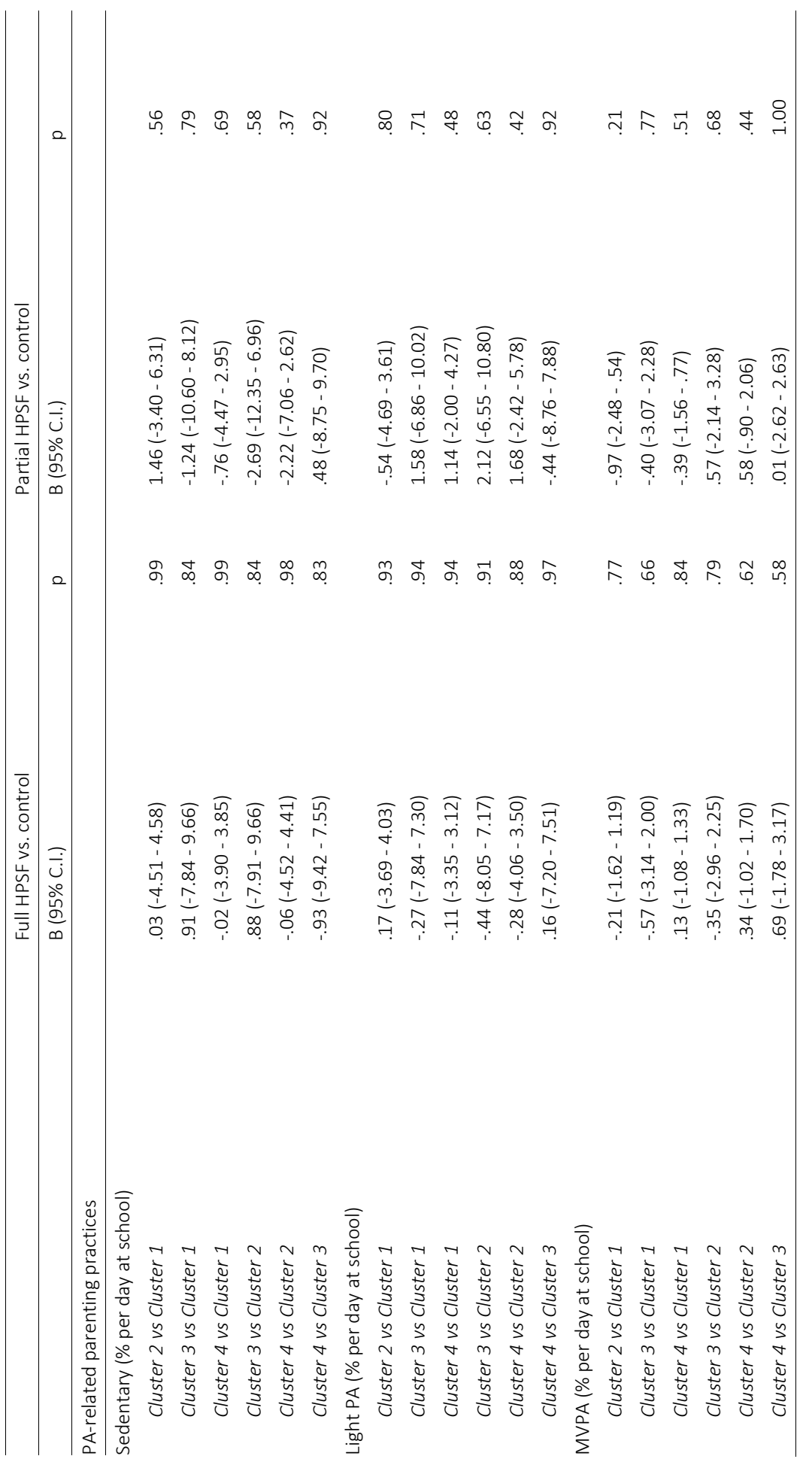




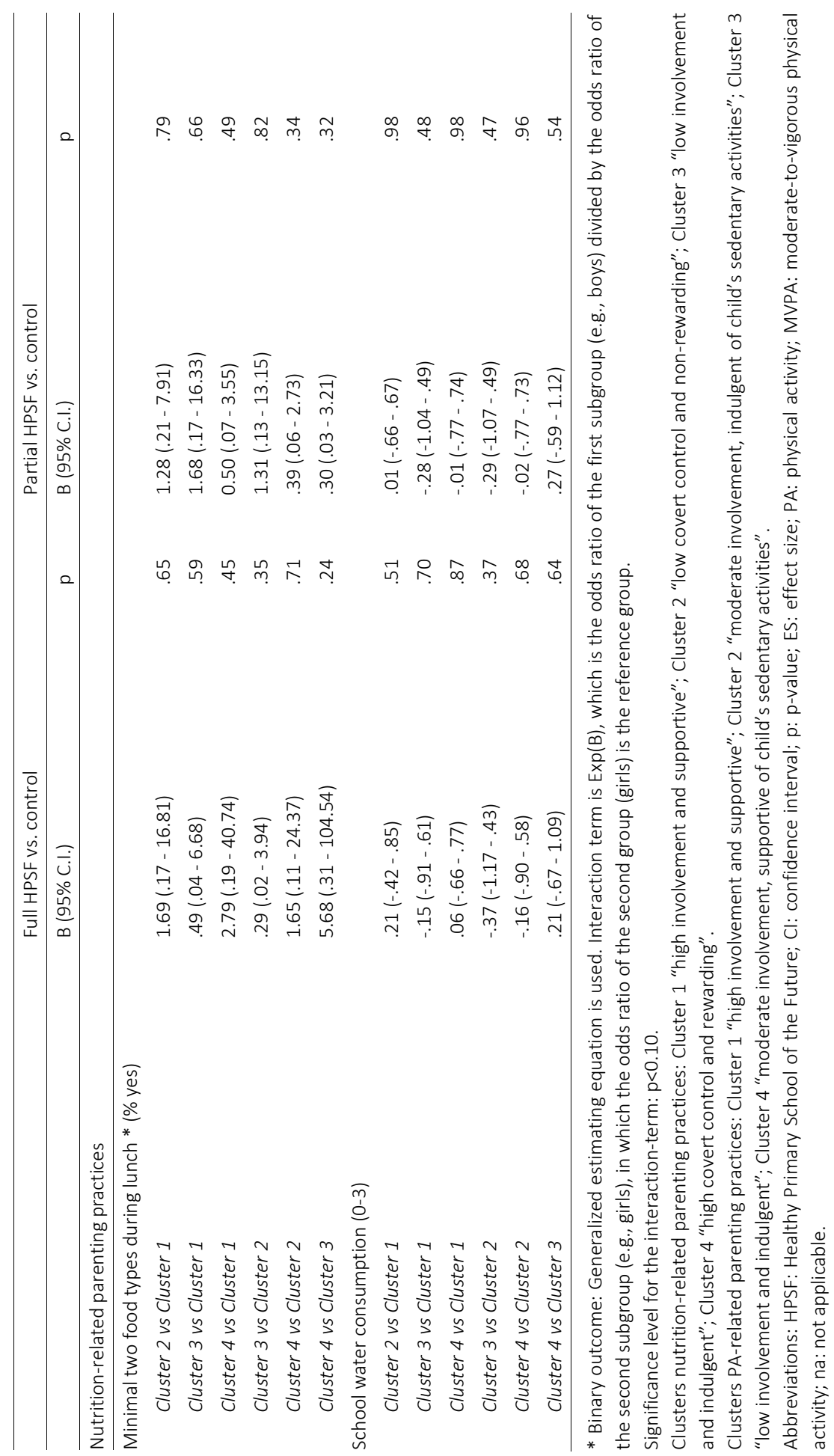




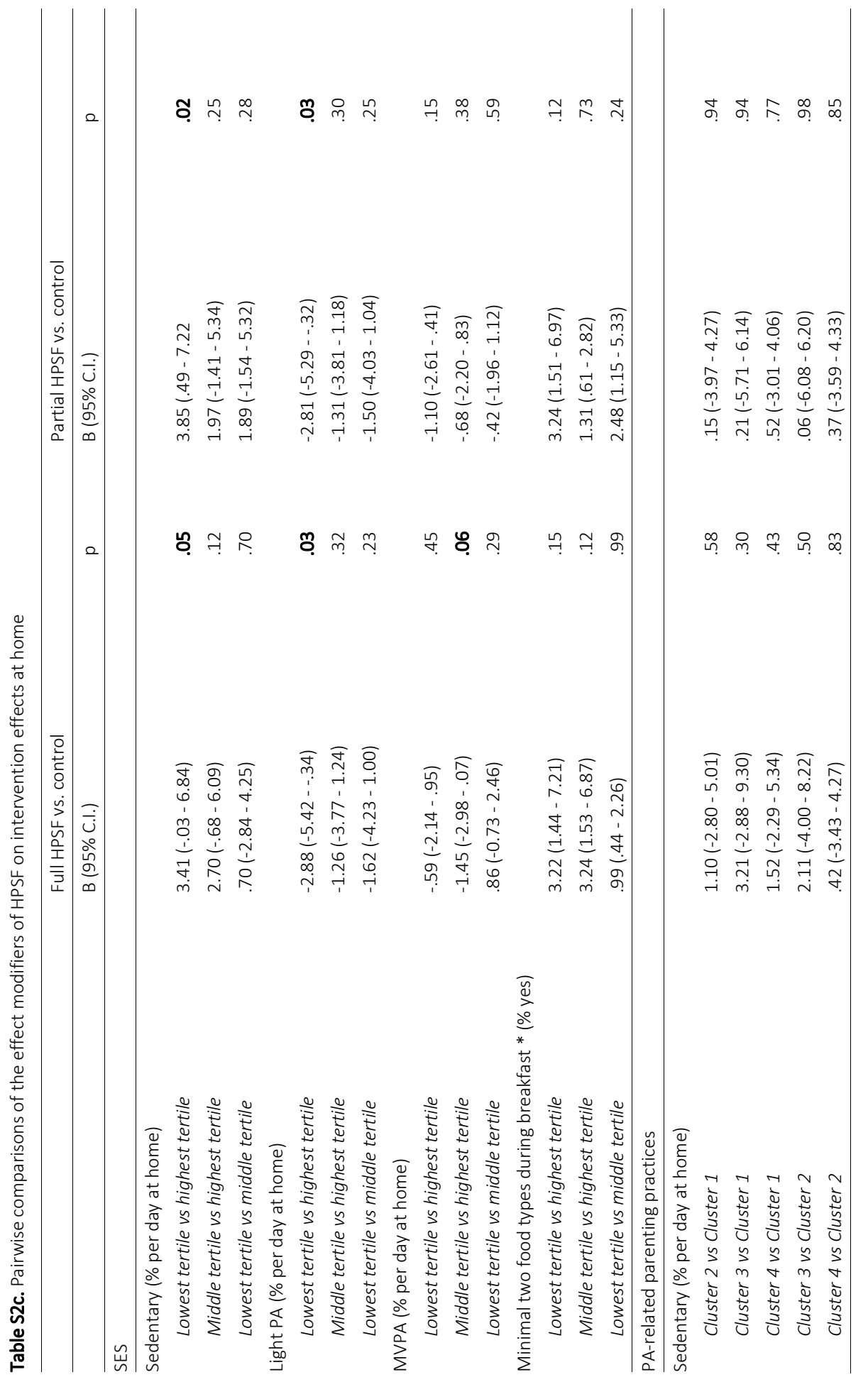


For whom and where is HPSF effective?

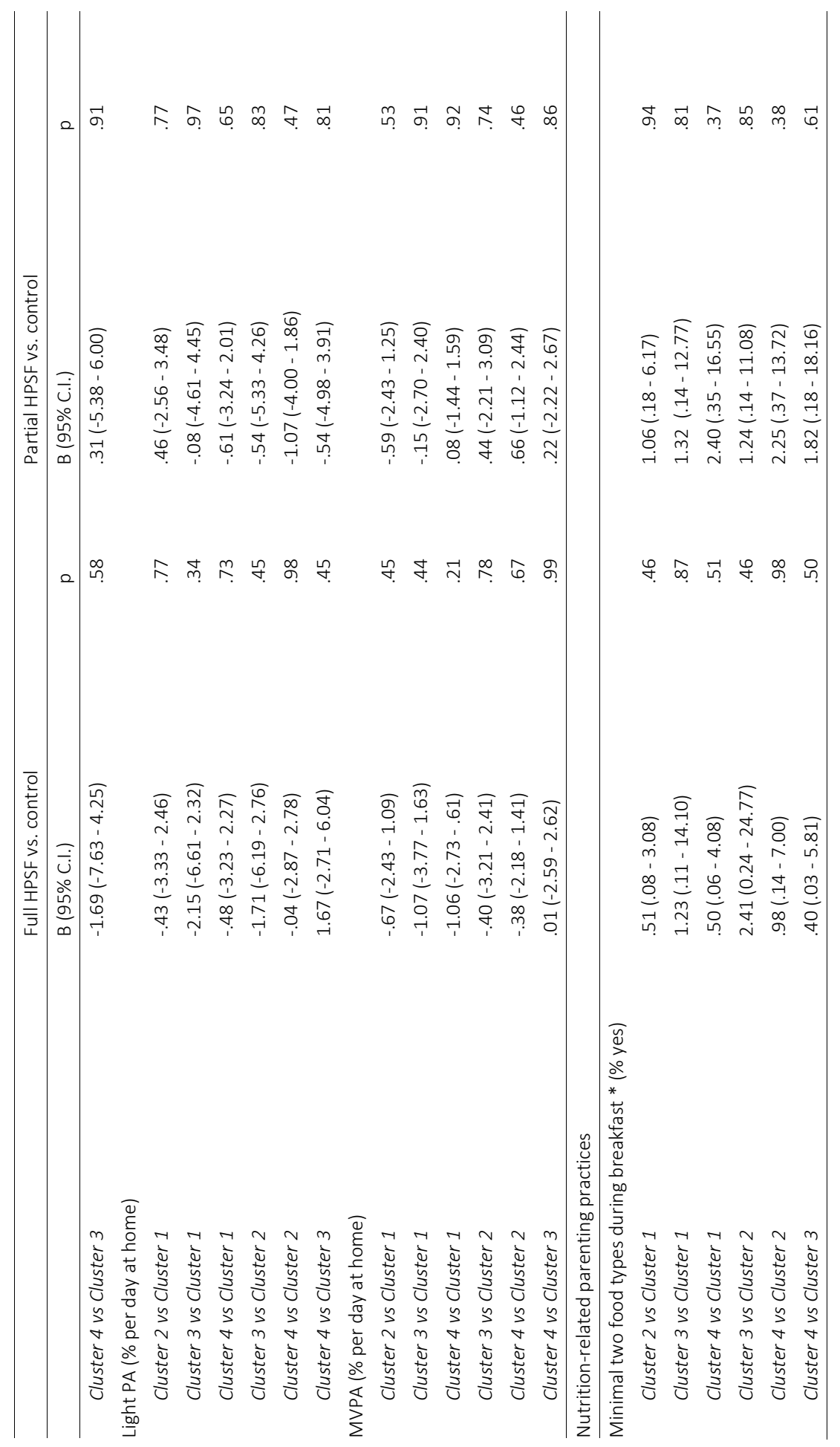


Chapter 6

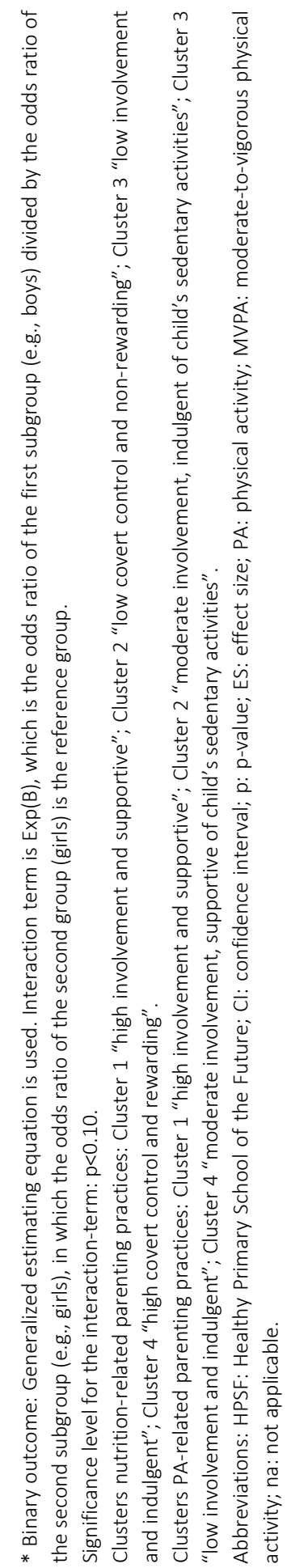






\section{Chapter 7}

The moderating role of the school context on the effects of the Healthy Primary School of the Future

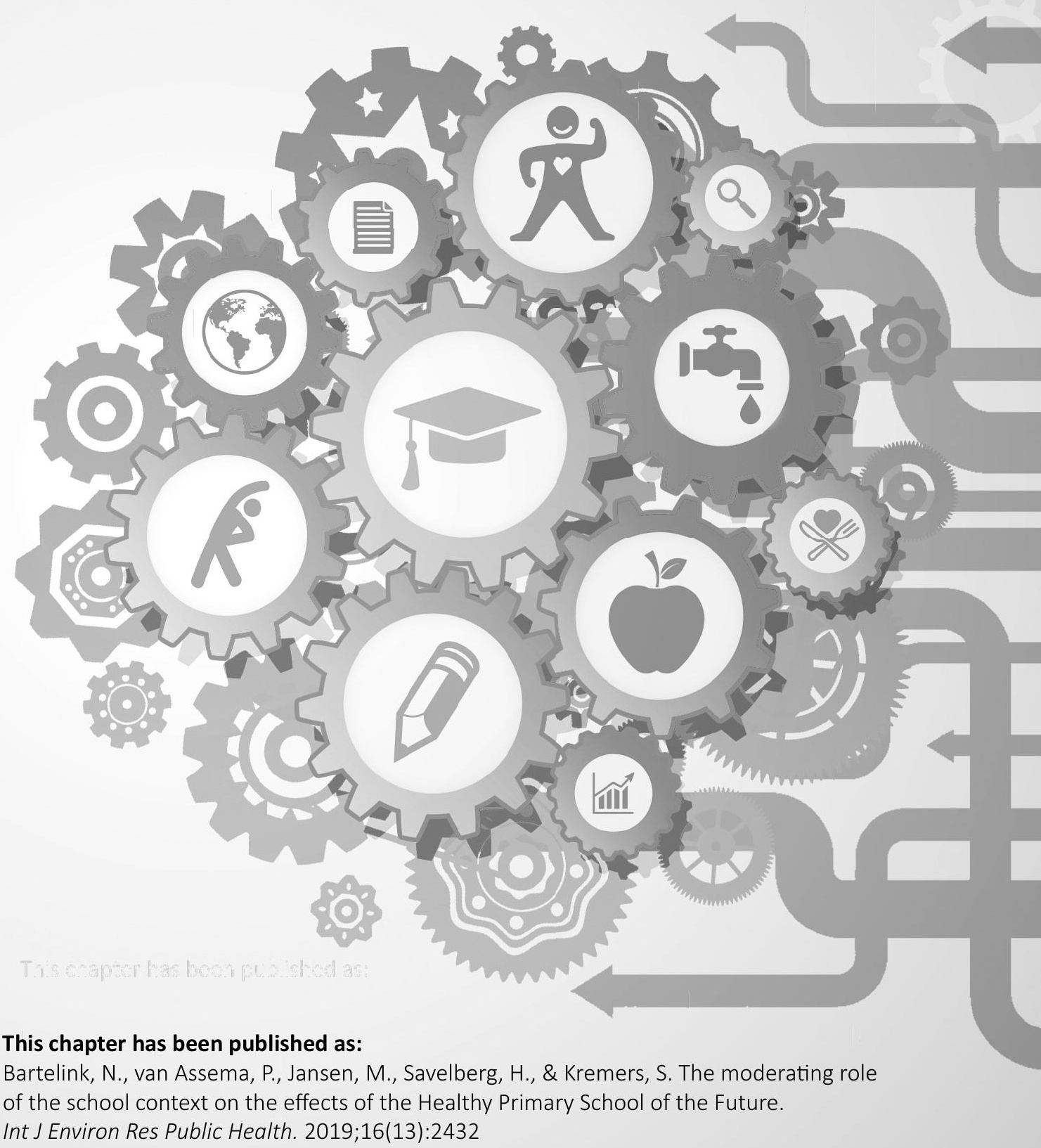




\section{Abstract}

\section{Background}

The current study investigated the moderating role of the school context on the effects of a Dutch health promoting school initiative on children's health and health behaviors.

\section{Methods}

The study used a mixed-methods design. The school context $(n=4)$ was assessed by the characteristics of the school population, teacher's health-promoting (HP) practices, implementers' perceived barriers, school's HP elements, and dominating organizational issues. Outcomes included objectively assessed BMI z-scores and physical activity (PA), and parent and child-reported dietary intake. Analyses included linear mixed models (four intervention schools versus four control schools), and qualitative comparisons between intervention schools with similar HP changes.

\section{Results}

Effects on outcomes varied considerably across schools (e.g., range in effect size on light PA of $0.01-0.26$ ). Potentially moderating contextual aspects were the child's socioeconomic background and baseline health behaviors; practices and perceived barriers of employees; and organizational issues at a school level.

\section{Conclusion}

Similar HP changes lead to different outcomes across schools due to differences in the school context. The adoption of a complex adaptive systems perspective contributes to a better understanding of the variation in effects and it can provide insight on which contextual aspects to focus on or intervene in to optimize the effects of HP initiatives. 


\section{Background}

Promoting healthy behaviors at an early age helps to improve children's health and their academic achievements [58, 87]. This may lead to improved health later in life and reduce the socioeconomic inequity in both health and academic achievement $[58,87]$. Schools have the potential to support children in improving their health behaviors [14, 15, 86]. However, school health promotion is often characterized globally by fragmentation, relatively low priority, and a lack of coordination [19, 25]. The Health Promoting School framework, as defined by the World Health Organization, aims for a whole-school approach and it focuses on embedding health and well-being in the curriculum, creating healthy social and physical environments, and engaging with parents and the wider community [21]. However, even though this strategy to integrate health promotion into the whole school system is promising, suboptimal results are often observed, due to, among other things, challenges regarding the implementation of specific health-promoting (HP) changes as part of this school-wide change and how to create a meaningful impact $[20,30,32,42,63,88]$. To understand these challenges, the suggestion has been made to consider schools as complex adaptive systems [11, 153]. A complex adaptive system can be described as a system that consists of many interacting components and has the capability to self-organize and adapt. The system's behavior is typically non-linear, not easily controlled or predicted, and it tends to selforganize to a state of stability [32, 41-45]. This means that each complex adaptive system acts in a unique way and can react differently to changes, since each one has its own context. Embracing this perspective of considering schools as complex adaptive systems means that it depends on the specific school context whether a specific HP change fits in a school, and that in each school, the implementation process of a specific HP change is different $[47,48]$. It also means that even when similar HP changes are achieved, these can have different effects across schools as the changes may be moderated by the unique context of the school $[48,61,154]$. Several studies have examined the role of the school context, but mainly focused on its interaction with HP changes during the implementation process $[42,155]$. The focus of this study was to examine the moderating role of the school context on the effects of HP changes when implementation is comparable between schools. This should contribute to a better understanding of the variation in effects of HP initiatives that is often found between schools [68].

School context is defined as the specific circumstances and characteristics of each school, which relates to the social, political, economic, and physical environment; the characteristics, behaviors, wishes, and needs of the people in the school; the wider community in which the school is located; as well as the history and organization of the school $[94,110]$. This definition shows that a school context consists of many different aspects. Previous studies have shown that some specific aspects might be of importance for school health promotion efforts. They include: characteristics of the 
school population (demographics, current health behaviors, health and well-being) [68]; HP practices of the teachers [42]; perceived barriers for implementation of HP initiatives, which can be categorized into barriers related to the users, the innovation, the support, the organization, and the socio-political environment [71]; current HP elements in the school (school routine, policy, education, and environment) [21]; and dominating organizational issues, e.g., merger process [68].

In a previous study, we developed a program theory (Figure 1) to visualize the interaction between the school context and the 'Healthy Primary School of the Future' (HPSF), which is a Dutch health promoting school initiative [110]. Part of this program theory concerns the moderating role of the school context on the effects of HPSF, as visualized by the moderator arrow in the top right of the model. The aim of the study was to explore the moderating role of the school context on the effects of HPSF among four primary schools (aged 4 to 12). Three research questions were formulated: 1) How did the school contexts differ from each other? 2) What are the effects of HPSF in each school on children's BMI z-score and their dietary and PA behaviors? and 3) Which aspects of the context relate to larger favorable effects of HPSF?

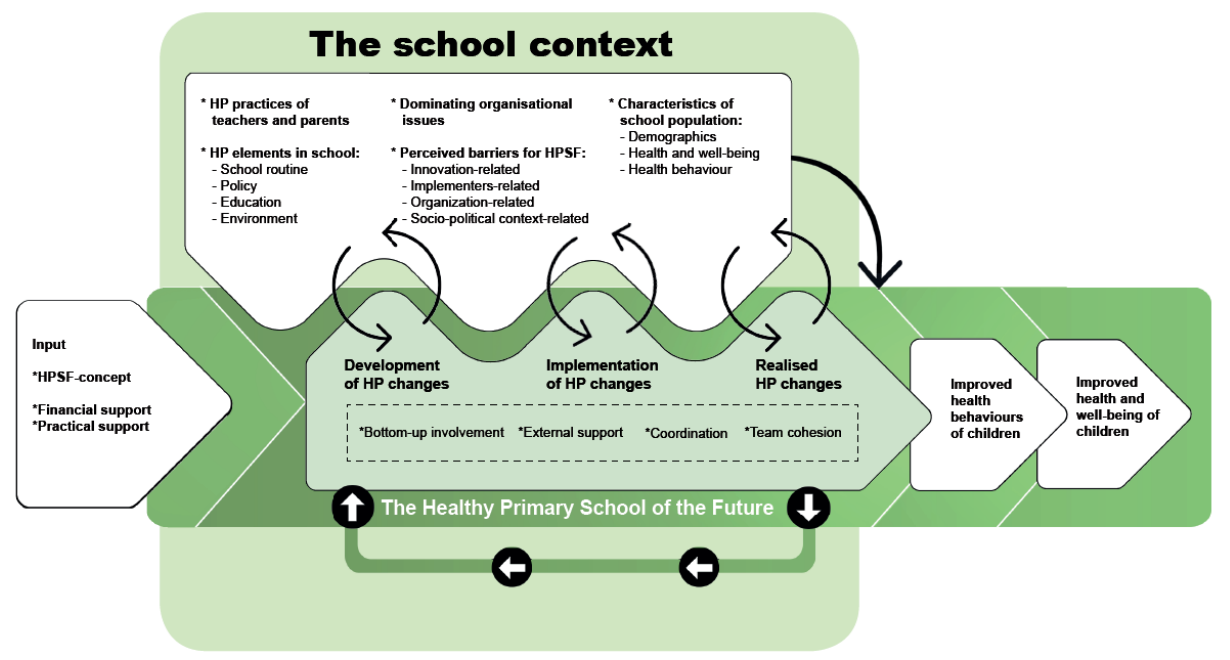

Figure 1. Program theory derived from Bartelink et al. [110]

The left side of the model shows the input, which is an 'event' that attempts to positively disrupt the preexisting dynamics in the school context to integrate health promotion. After the introduction of HPSF into the school context, the process of development, implementation, and integration of HP changes develops in the school. During this process, it is hypothesized that HPSF will continuously interact with the school context. The loop in the bottom of the program theory visualizes the key assumption that realized changes may shift the school's norms toward a focus on health and well-being, thereby creating momentum for additional HP changes. Overall, the process of change should lead to the realization of HP changes that fit the school's context. The combination and interaction of all these contextualized HP changes should impact children's health behaviors and, through this, their health and well-being. A key assumption in the cause-effect relation concerns non-linearity: it is assumed that small changes in a school can produce large effects at a so-called 'tipping' point. The arrow in the top right of the model visualizes the moderating role of the school context. The key assumption is that even when a change is similar, the school context will determine its impact. 


\section{Methods}

\section{The Healthy Primary School of the Future}

HPSF is a Dutch initiative that aims to sustainably integrate health and well-being within the whole school system. Three cooperating organizations developed the idea for HPSF: the regional educational board 'Movare', the regional Public Health Services, and Maastricht University [55]. HPSF is based on the principles of the Health Promoting School framework and intends to establish a broad collaboration between the school, parents, and external partners, to develop and implement HP changes in the whole school system, e.g., the school's physical and social environment, its health policy, education, and routines [55, 92]. On top of the Health Promoting School framework, the initiative aims to create some form of positive disruption in the school, by initiating two changes top-down: 1) a free healthy lunch each day and 2) structured PA and cultural sessions after lunch, both led by external pedagogical employees provided by childcare organizations. These two changes should create momentum for bottom-up processes to implement additional HP changes [92]. Each school selected a teacher as the school coordinator, who managed HPSF in their school. Overarching the schools, the HPSF initiative was led by a project leader from Movare and an executive board with representatives from the three collaborating organizations, including the project leader. A project team was created with representatives of all the partners involved: the four schools, Movare, regional Public Health Services, Maastricht University, childcare organizations, sports and leisure organizations, a caterer, and the Limburg provincial authorities.

Four intervention schools participated in HPSF and started implementing HP changes in November 2015. Since the schools themselves decided on the adoption and implementation of HP changes, some differences existed between them. School 1 (S1) and School 2 (S2), referred to as the 'full HPSF', decided to implement the two topdown changes, i.e., the lunch and the structured PA and cultural sessions [110]. To realize these changes during the lunch break, both schools extended the lunch break period by about 60 minutes. Therefore, children attended school to approximately 15:30/15:45 instead of 15:00. Both schools also implemented several additional HP changes, that is, they both provided water bottles to all children, improved their school's health policy, and started with an educational lunch. The two schools implemented all HP changes in a comparable way and had similar support from external partners [110]. School 3 (S3) and School 4 (S4), referred to as the 'partial HPSF', decided to only implement the structured PA and cultural sessions each day. They did not provide a healthy lunch nor did they increase their lunch break time or implement additional HP changes [110]. The effects of the full and partial HPSF after a one- and two year follow-up were investigated in two previous studies [111, 124]. Significant favorable intervention effects after one- and two years' follow-up were found for the 
full HPSF on children's dietary behaviors for, among others, school water consumption, lunch intake of vegetables and dairy products. Children's sedentary time and light PA significantly improved after two years' follow-up. Almost no significant favorable results on children's health behaviors were found in the partial HPSF. In addition, results have shown that children's BMI z-scores in both the full and the partial HPSF significantly decreased after two years' follow-up. This favorable effect was already significant after one year's follow-up in the partial HPSF, but not yet in the full HPSF.

\section{Study design}

The current study is part of an overall study investigating HPSF, which included the four intervention schools and four control schools [55]. All the schools are members of the regional educational board 'Movare' situated in the Parkstad region in the southern part of the Netherlands. This region is characterized by a low average socioeconomic status, and unhealthy behaviors and overweight are highly prevalent compared to the rest of the Netherlands $[40,62]$. Ethical approval (14-N-142) for the overall study was given by the Medical Ethics Committee Zuyderland, located in Heerlen (Parkstad, the Netherlands). The current study incorporated two different study designs which were previously used in the overall study: 1) A longitudinal quasi-experimental study design to investigate the effects in each school [111, 124], and 2) a mixed-methods study design to assess the four schools' context and its moderating role [110]. Measurements for the quasi-experimental study were conducted during one week of measurements from September-November of 2015 (T0), 2016 (T1) and 2017 (T2). All children (aged 4 to 12 ) and their parents ( $n=2326$ at T0) from the eight schools were invited to participate in the study. All the participants were required to complete an informed consent form, signed by (both) parents. In the mixed-methods study, a contextual action-oriented research approach (CARA) was used as in Reference [92], which focused on contextual differences and the use of monitoring and inducing feedback loops to support and evaluate the processes of change. Data were collected in the four intervention schools over three years (2014-2017), that is, the development year (20142015) and the first two years of implementation (2015-2017) of the HPSF. The overall consent of the schools' employees (school coordinator, teachers, external pedagogical employees) was obtained by consent of the director of the school.

\section{Measures}

\section{Effect measures}

Children's BMI was assessed by anthropometric measurements of height and weight during physical education lessons. BMI z-scores were calculated using Dutch reference values as in Reference [5]. Children's PA behaviors were assessed using accelerometry (Actigraph GT3X+, Pensacola, FL, US, 30Hz, 10s epoch). The activity levels, in counts-per- 
minute (CPM), were classified using Evenson's cut-off points [138]: sedentary behavior (SB; $\leq 100$ CPM), light PA (LPA; 101 - 2295 CPM), and moderate-to-vigorous PA (MVPA; $\geq 2296$ CPM). Children's dietary behaviors were assessed through questionnaires addressed to the parents and children. The measures of the parents' questionnaire were combined into two total scores (mean days/week): one for healthy dietary behaviors (breakfast, fruits, vegetables, and water), and one for unhealthy dietary behaviors (sugar-sweetened beverages and snacks). Two children's questionnaires were used to obtain information about children's school water consumption and their lunch intake. The intake of specific food types (grains, butter, dairy, fruits, vegetables, and water) were summed, and a dichotomous variable was created to study whether children consumed at least two of the food types during lunch. A more detailed description of the data collection procedures and the specific effect measures has been reported in the effect evaluation studies $[111,124]$.

\section{Context measures}

Characteristics of the school population

The number of children and teachers in each school was obtained, and their demographics and starting situation regarding health and health behaviors were assessed. The number of children in each school, their gender, ethnicity, and study year at baseline were collected from the database of the educational board Movare. Children's ethnicity was determined by the country of birth of both parents and divided into: (1) Western background (including the Netherlands) and (2) non-Western background as described in Reference [114]. If one or both of the parents was born in a non-Western country, the child's ethnicity was assigned to non-Western. A digital questionnaire for the parents was used to obtain information about the children's socioeconomic status (SES), which was calculated as the mean of standardized scores on maternal education level, paternal educational level, and household income (adjusted for household size) [113]. The mean scores were categorized into low, middle, and high SES scores based on tertiles. To examine the children's starting situation on health and health behaviors, the mean baseline scores per school of the abovementioned effect measures were used. The number of teachers in each school was obtained from the school coordinators. Demographics and the starting situation of the teachers were collected from the teachers themselves by including additional questions on the HP practices questionnaire (see next section) regarding their gender, date of birth, the number of years employed by the school, and their height and weight. The latter was used to calculate their BMI.

\section{HP practices of the teachers}

A paper-based questionnaire was used to gain insight into the nutrition-related and PArelated HP practices of teachers at school, e.g., modelling behavior and involving children in nutrition or PA-related activities. The questionnaire was based on previous 
work by Gevers et al. [81, 98] and O'Connor et al. [80], in which acceptable to good testretest reliability of their instruments was found. The questionnaire was filled out annually by teachers at the beginning of the school year and consisted of 30 items (13 nutrition-related practices and 17 PA-related practices). Each item described a practice using a statement, followed by some examples. Participants responded on a Likert scale from 1 (completely disagree) to 5 (completely agree).

\section{Perceived barriers to the implementation of HP changes}

To gain insight into the perceived barriers to the implementation of HP changes, a 46item questionnaire was used. The questionnaire was distributed by e-mail and all implementers, i.e., teachers and external pedagogical employees, were asked to complete it digitally or in writing. The questionnaire was completed twice a year; for the current study, we included the data obtained prior to the start of the HPSF (TO) and after two years of implementation (T2). The questionnaire was based on the Measurement Instrument for Determinants of Innovations (MIDI), a Dutch questionnaire developed by Fleuren et al. [156]. They developed it through a systematic review of empirical studies and a Delphi study amongst implementation experts. The questionnaire has been used in many different implementation studies, especially in the school setting, although no specific research has been conducted to evaluate its validity and reliability [71]. Items were formulated as a statement, and responses to each statement ranged from 1 (totally disagree) to 10 (totally agree). The items were related to possible barriers regarding: (a) the users, i.e., the implementers themselves $(n=13)$, (b) the innovation, i.e., the HP changes $(n=7),(c)$ the support $(n=9)$, (d) the organization, i.e., the school $(n=13)$, and (e) the socio-political environment $(n=4)$. For each category, a mean score was calculated (maximum two missing).

\section{HP elements in the school}

In the HPSF research, we used the term HP elements for initiatives in the school that potentially add to school-wide health promotion. A short questionnaire was filled out in the four intervention schools to gain insight into all these HP elements. Prior to the start of the HPSF (TO), it was done by interviewing the HPSF school coordinator, after two years (T2), the school coordinators filled out the questionnaire themselves. The HP elements were divided into four themes: school routine, policy, education, and the environment. Elements regarding school routine $(n=7)$ were determined using questions on the use of energizers, drinking water during classes, the lunch in school, PA after lunch break, PA after school, the existence of working groups, and the involvement of parents. Elements regarding policy $(n=7)$ were determined using questions on rules and policy on snacks, lunch, treats, sugar-sweetened beverages, sport and energy drinks, water, and special policy on school events. Elements regarding education $(n=7)$ were determined using questions on having an educational lunch, swimming lessons, the number of minutes per week of physical education classes, and 
the use of four specific classroom-based programs regarding a healthy lifestyle. Elements regarding environment $(n=7)$ were determined using questions on the presence of a school vegetable garden, a bicycle parking area, a sports hall in the neighborhood, the use of volunteers to help children crossing a busy road, having a safe route to school, having an active schoolyard, and whether the schoolyard is open after school hours. The results were combined and translated into an overall score for that theme to indicate the extent to which it was present in the school (absent (-), minimally present $(X)$, moderately present $(X X)$, or largely present $(X X X)$ ).

\section{Dominating organizational issues}

A dominating organizational issue can be anything that could distract a school's focus from its regular work and the implementation of the HPSF, e.g., staff turnover. Insight into existing dominating organizational issue(s) in the four schools was gained using several methods. The annual interviews with the HPSF school coordinators provided insight, and open questions were added to the barrier questionnaire, e.g., 'Do other issues in school exist that influence the implementation of the Healthy Primary School of the Future?' Furthermore, minutes of HPSF meetings that were held on an overarching level or on a school level, as well as formal and informal talks with people in the schools provided insight into any existing dominating organizational issue. The dominating organizational issues were indicated per school as absent (-) or present (X).

\section{Analyses}

The analyses were conducted in five steps to investigate the four school contexts, the effects of HPSF in each school, and whether aspects in the context related to larger favorable effects.

\section{Step 1: Assessing the four school contexts}

Descriptives were used for the quantitative context measures. The qualitative context measures were described on whether they were present in each school context and to what extent. The specific context in each school was assessed by comparison with the other schools.

Step 2: Comparing the school contexts between the schools with similar HP changes Aspects in the school context were compared between the schools with similar HP changes. This meant that we compared the context of the full HPSF schools, i.e., S1 versus S2, and the context of the partial HPSF schools, i.e., S3 versus S4. Major differences between the contexts were described. 
Step 3: Assessing the effects of HPSF in each school

IBM SPSS Statistics for Windows (version 23.0, IBM Corp, Armonk, NY, USA) was used to analyze the effects of HPSF in each school. Linear mixed-model analyses were conducted for the continuous effect measures and generalized estimating equations for the binary effect measures. These analyses, as well as the imputation method to handle missing data, were similar to the studies in which the effects of the full and partial HPSF on children's BMI z-score and health behaviors were investigated [111, 124]. A twosided $p$-value $\leq 0.05$ was considered statistically significant. Standardized effect sizes (ES) were determined for continuous effect measures, which were computed as the pooled estimated mean difference divided by the square root of the pooled residual variance at baseline. Odds ratios $(O R)$ were determined for the binary effect measures.

Step 4: Comparing the effects of HPSF between the schools with similar HP changes The effect sizes/odds ratios of all effect measures derived from Step 3 were compared between S1 and S2, and between S3 and S4. Similarities and differences were described.

Step 5: Exploring whether aspects in the school context relate to larger favorable effects of HPSF

This step was based on the principles of qualitative comparison analysis (QCA) [157, 158]. QCA is a case-oriented approach that examines which aspects, alone or in combination with other aspects, are necessary or sufficient to produce an outcome. Using the principles of QCA, we aimed to explore whether aspects in the context relate to the larger favorable effects of HPSF. The findings from Step 2 and 4 were combined to conduct this step.

\section{Results}

The results are described according to the five steps of analysis. Table 1 presents all the results.

\section{Step 1: Assessing the four school contexts}

\section{School population}

S1 was characterized by a school team with the highest number of teachers, as well as having the highest mean age of teachers. The children in this school had the most favorable starting situation regarding the BMI z-score, i.e., lowest z-scores, as well as PA behaviors, i.e., most time spent in PA. However, regarding children's dietary behaviors, they had the least favorable starting situation. S2 was characterized by the least favorable starting situation of teachers, that is, their self-reported BMI was the highest 
compared to the teachers in the other schools. In S2 and S3, the school population, both children and teachers, was smallest at the start of the HPSF and it included the highest percentage of children who were part of the low SES tertile. Furthermore, S3 was characterized by the highest percentage of children with a Western ethnicity and the most favorable starting situation of children regarding their dietary behaviors. The school team in S3 consisted completely of female teachers, and they had been employed in their school for the shortest amount of time compared to the teachers in the other three schools. S4 is characterized by the highest number of children, as well as having the lowest mean age of children.

HP practices of teachers

S1 had the most and largest improvements in teachers' PA-related practices. S2 had the most and greatest improvements in the teachers' nutrition-related practices, and they were also the most favorable at T2 compared to the other schools. In S3, the teachers' PA-related practices at the start of HPSF were the most favorable and many practices remained the most favorable at $\mathrm{T} 2$. In $\mathrm{S} 4$, the nutrition-related practices were the least favorable at both T0 and T2.

Perceived barriers to the implementation of HP changes

Teachers in S1 perceived the most barriers to implementation at both T0 and T2. External pedagogical employees in S1 perceived the least barriers to implementation of the HP changes. The opposite was observed in S3, where teachers perceived the least barriers and external pedagogical employees the most barriers. More detailed results, i.e., the results on each specific barrier in each school, were reported in Bartelink et al. [110].

HP elements in school

S1, S2, and S4 had limited HP elements at TO. S1 and S2 had improved greatly on all aspects at T2: policy, education, the environment, and school routine; whilst the improvements in S4 were limited. In S3, several HP elements existed already at the start of the HPSF. They did not show much improvement at T2. More detailed information on the specific HP elements in each school was reported in Bartelink et al. [110].

\section{Dominating organizational issues}

S1, S2, and S3 had to deal with a dominating organizational issue. S1 arose from the merger of two separate schools at the start of the HPSF, as well as having moved to a new school building. This merger process created a new way of working in the school. S2 had to deal with a merger as well. This merger was realized in September 2016, after the first year of the HPSF. For this merger, the school building had to be renovated, so they had to move to a temporary location with limited PA possibilities in and around the school, for the first year of the HPSF. This temporary location limited the 
implementation of HPSF. S3 had to deal with a major staff turnover at the start of the HPSF. This turnover had contributed to the decision of the school not to provide a healthy lunch.

Step 2: Comparing the school contexts between the schools with similar HP changes

The full HPSF: S1 versus S2

Differences were observed in four of the five contextual aspects.

School population: A larger percentage of children in S2 were part of the lowest SES tertile (32.3\%), compared to S1 (24.0\%). Children's starting positions differed as well: the children in S1 had a more favorable mean BMI z-score (S1: mean (standard deviation (SD)) 0.028 (1.00) versus S2: 0.092 (1.02)) and they were more physically active (e.g., light PA in S1: (mean \% per day (SD)) 32.8\% (5.48) and in S2: 30.6\% (5.98)). Dietary behaviors (in school) were more favorable in S2 (e.g., healthy behaviors in S1: mean (SD) 5.06 (1.16) and in S2: 5.33 (1.05); minimum of two food types during lunch in S1: $78.7 \%$ and in $52: 84.6 \%)$.

HP practices of teachers: The greatest and most improvements in PA-related teacher practices were found in S1 (e.g., encouragement in S1: TO (mean (SD)) 4.2 (0.71), T2 4.6 (0.57)) and in S2 (TO 4.5 (0.64), T2 4.4 (0.61)) and in nutrition-related practices in S2 (e.g., healthy modelling in S2: T0 4.3 (1.10), T2 4.7 (0.56)).

Perceived barriers to the implementation of HP changes: The external pedagogical employees in S1 perceived the least barriers at T0 and T2, but in S2, the greatest improvements in perceived barriers could be observed over the two years (e.g., innovation-related barriers in S1: TO (mean (SD)) 7.6 (1.42), T2 $7.6(0.60)$, and in S2: TO 6.9 (0.20), T2 7.3 (1.04)).

Dominating organizational issues: Even though both S1 and S2 had to deal with a merger process, the impact was different, i.e., in S1, it reinforced the implementation of HPSF, whilst in S2, it limited the implementation.

HP elements in school: No differences were observed in this contextual aspect.

The partial HPSF: S3 versus S4

Differences in context were observed in three of the five contextual aspects.

School population: A larger percentage of children in S3 $(38.4 \%)$ were part of the lowest SES tertile compared to S4 (28.1\%). The starting situation of the children was also different: more favorable in S3 regarding BMI z-score (S3: 0.082 (1.01) versus S4: 0.099 (0.91)) and dietary behaviors (e.g., school water consumption in S3: (mean (SD)) 2.94 (1.22) and in S4: 1.93 (1.06)), and more favorable in S4 regarding PA behaviors (e.g., sedentary time in S3: (mean \% per day (SD)) 61.6\% (6.54) and in S4: 60.3\% (7.29)). Furthermore, teachers in S3 had been employed in their school for a shorter amount of time (mean (SD): 0.92 (1.24)) compared to those in S4 (12.64 (8.56)). 
HP practices of teachers: Teacher's PA practices at the start of HPSF were more favorable in S3 than in S4 (e.g., involving children in PA activities was in S3: 4.5 (0.53) and in S4: 3.5 (1.03)).

Perceived barriers to the implementation of HP changes: A larger decline in perceived barriers of teachers was observed in S3 compared to S4 over the two years (e.g., support-related barriers in S3: TO 7.1 (1.80), T2 8.6 (0.65) and in S4: TO 7.5 (0.87), T2 $8.0(0.94))$. External pedagogical employees of S4 perceived fewer barriers at both time points compared to S3 (e.g., user-related barriers in S3: TO 7.5 (0.68), T2 7.2 (0.55) and in S4: TO $7.6(0.65)$, T2 $7.9(0.38))$.

Step 3: Assessing the effects of HPSF in each school

The largest effects on children's BMI z-score were found in S1 (ES=-0.11) and the smallest in S3 (ES=-0.04) (Table 1; Table S1 in Additional file 1). For the effects on children's PA behaviors, i.e., the time children spent sedentary, in light PA and in MVPA, the largest effects were found in S1 and the smallest in S3. The effect in S4 on the time children spent in MVPA was comparable to S1 (ES=0.15). Overall dietary behaviors improved most in S1, i.e., an increase in healthy dietary behaviors $(E S=0.25)$ and a decrease in unhealthy dietary behaviors (ES=-0.13). The least favorable effect on healthy dietary behaviors, with a negative effect size, was found in S4 (ES=-0.08). Regarding unhealthy dietary behaviors, an adverse effect was also found, that is, in S3, the ES was 0.06. The largest effects were found in S2 on school dietary behaviors, i.e., school water consumption ( $E S=1.17$ ) and the intake of at least two healthy food types during lunch at school $(\mathrm{OR}=3.96)$. The least favorable, and even adverse, effects were found in S3 (school water consumption: $E S=-0.20$; lunch intake: $O R=0.20$ ).

Step 4: Comparing the effects of HPSF between the schools with similar HP changes

The full HPSF: S1 versus S2

Larger favorable effects were found in S1 compared to S2 for children's BMI z-score, their PA behaviors and their overall dietary behaviors. Looking at the effects on dietary behaviors in school, the effects were similar or more favorable in S2.

The partial HPSF: S3 versus S4

The favorable effects on all outcome measures were larger in S4 compared with S3, except for overall healthy dietary behaviors. 
Step 5: Exploring whether aspects in the school context relate to larger favorable effects of HPSF

Five aspects in the context appeared to be related to larger favorable effects. Larger effects were found in schools with: (1) fewer children in the lowest SES tertile; (2) more favorable starting positions of children regarding their health behaviors; (3) most improvements in nutrition and/or PA-related practices of teachers, specifically related to modelling and encouragement; (4) least barriers perceived by the external pedagogical employees; and (5) in the schools that used the opportunity created by a dominating organizational issue, e.g., merger process, to synergize it with the implementation of the HPSF. 
Moderating role of the school context

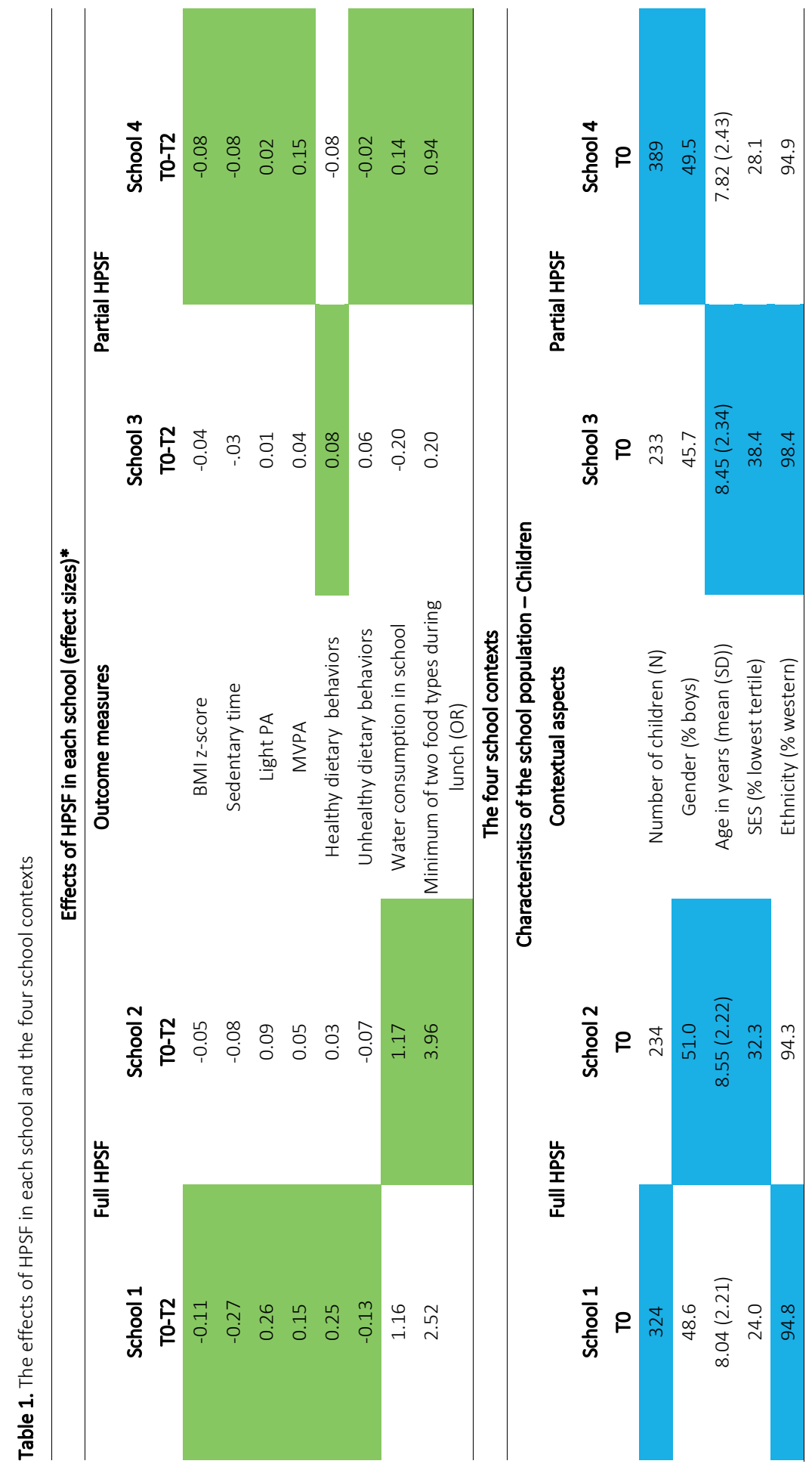




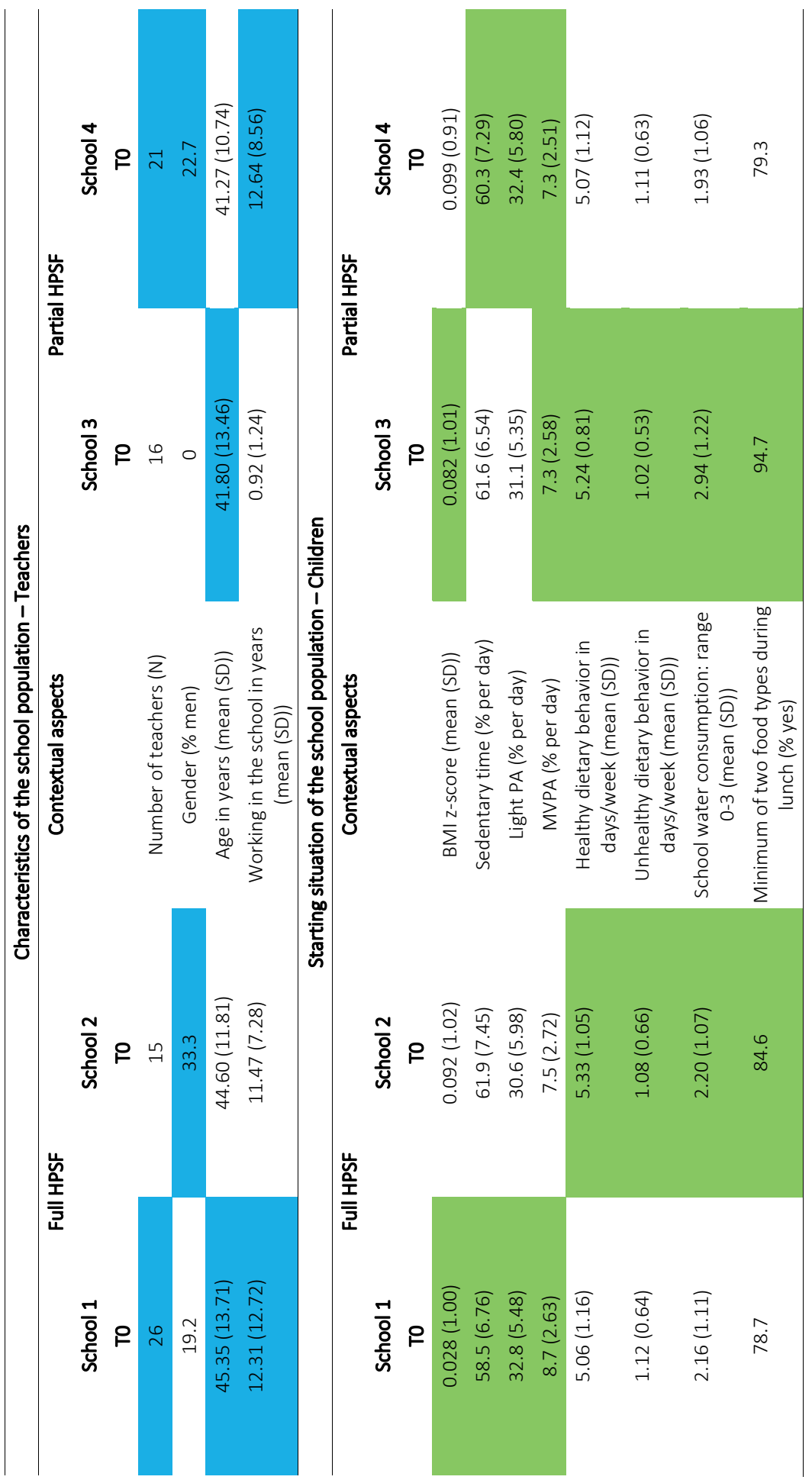




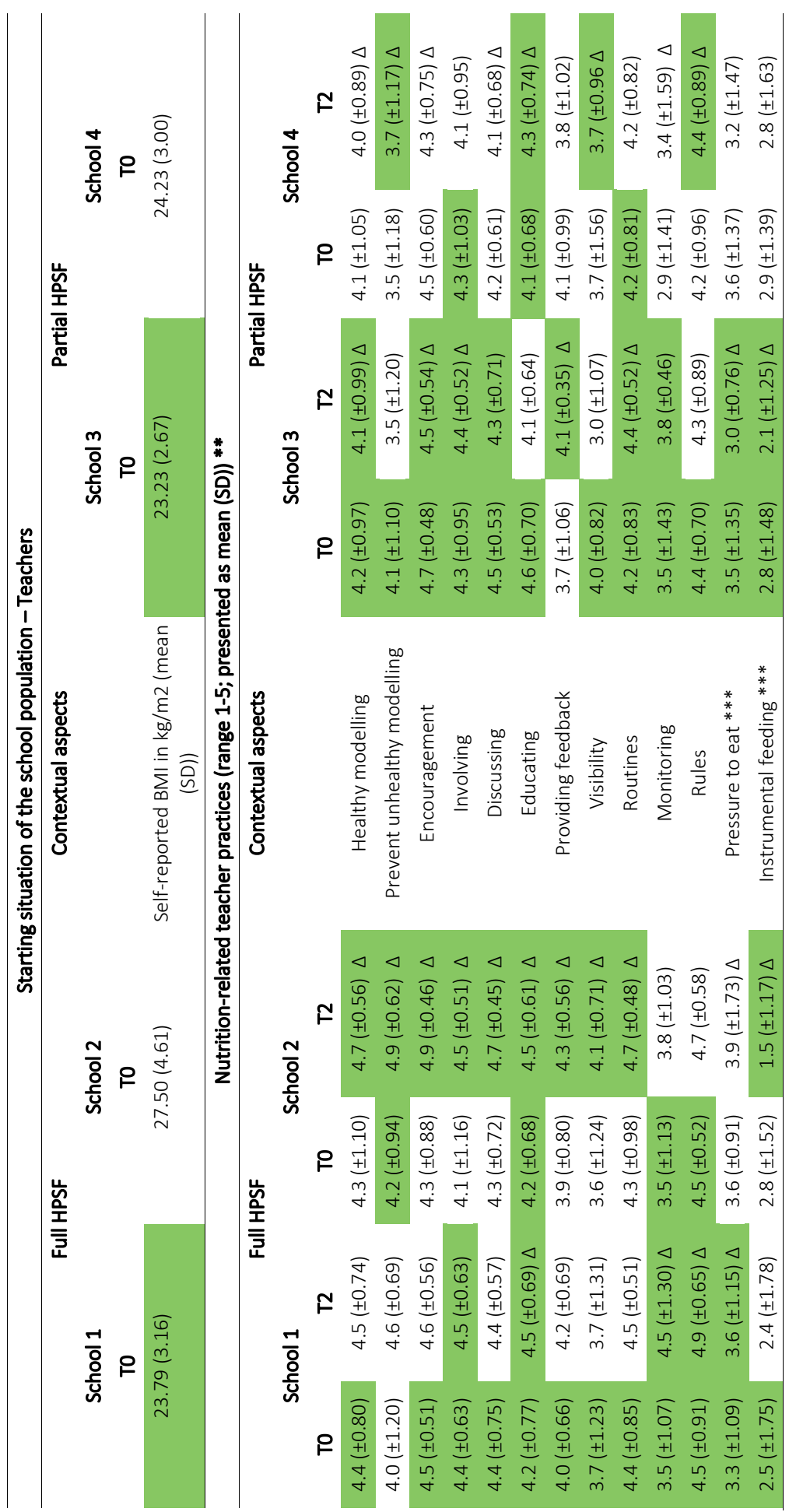




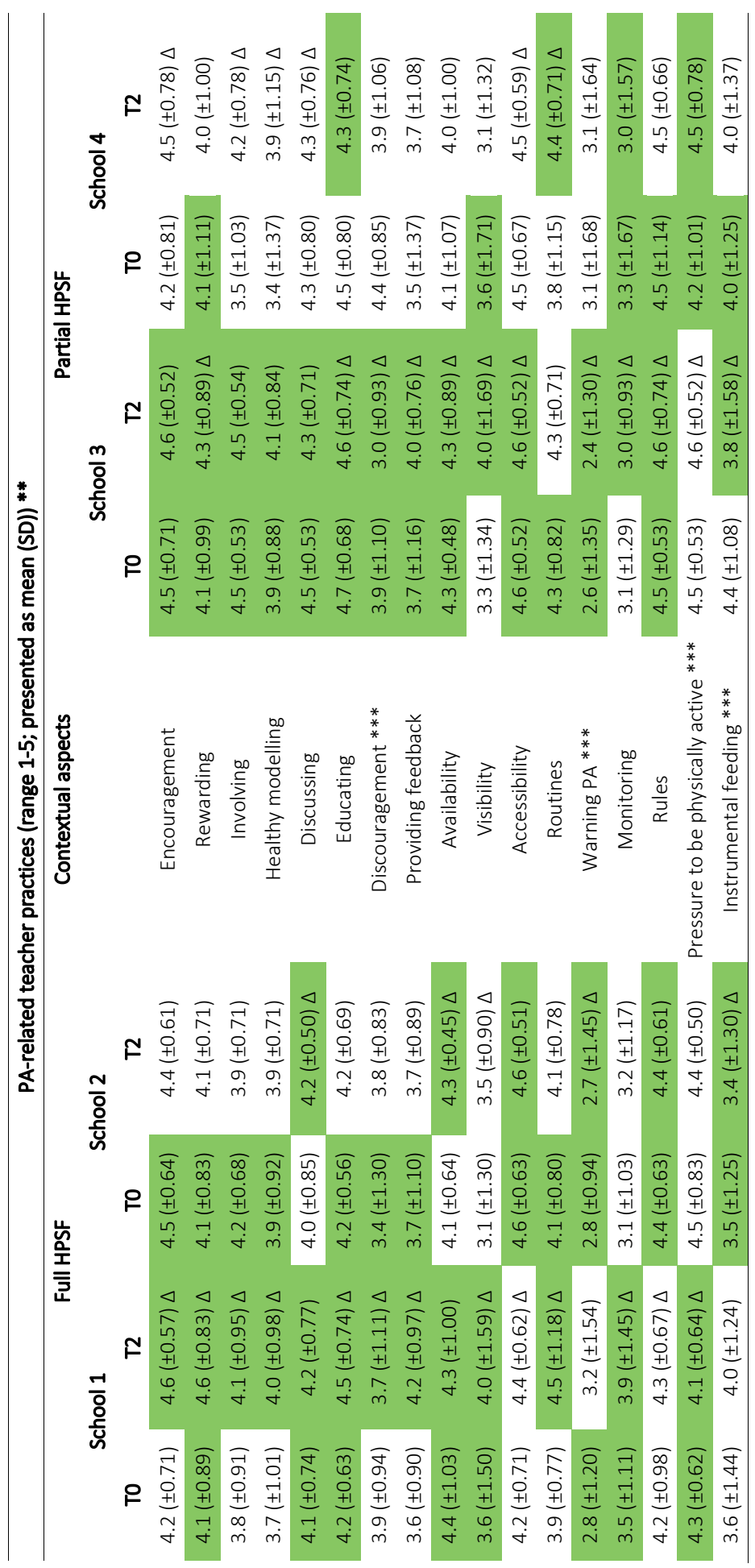




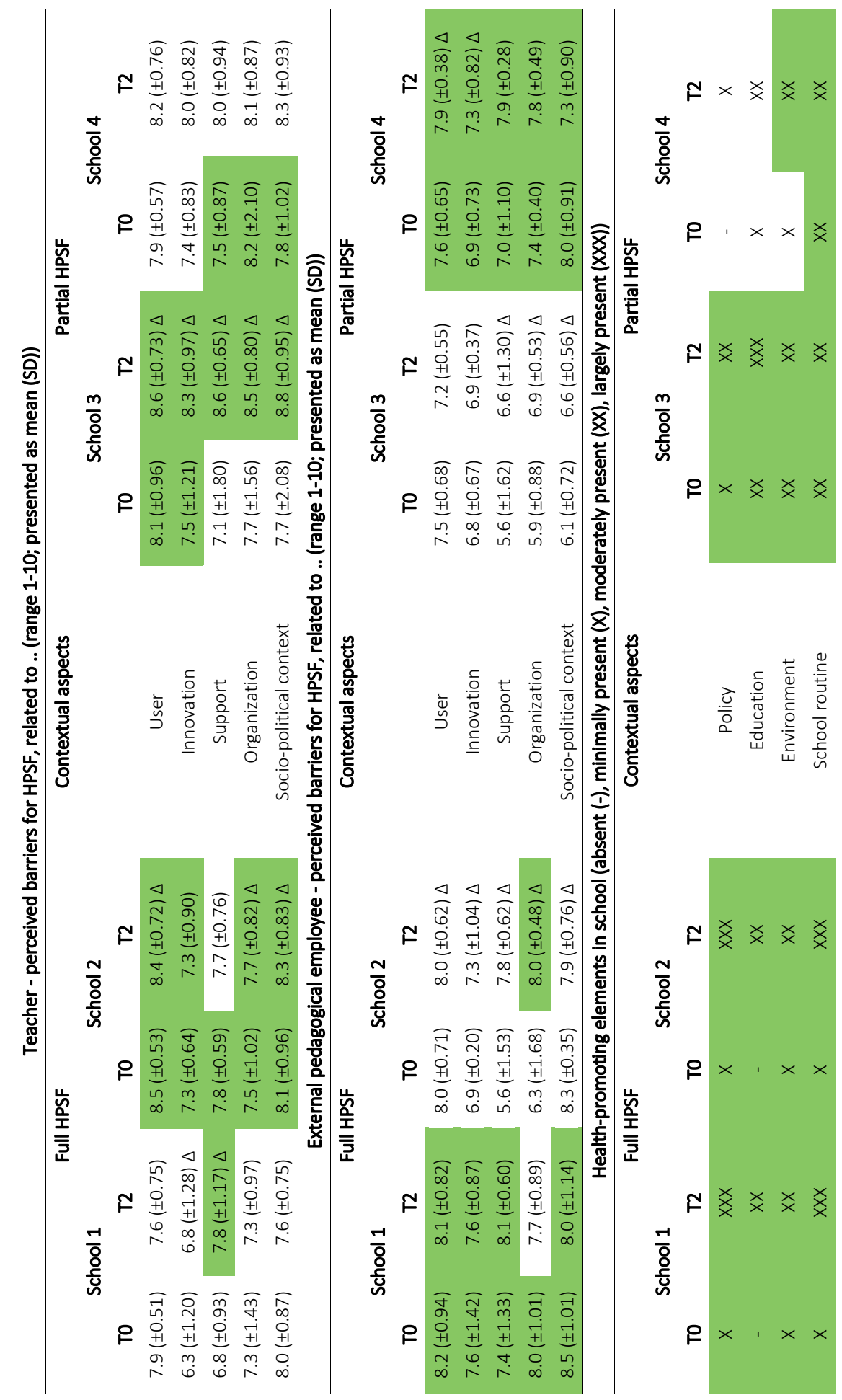




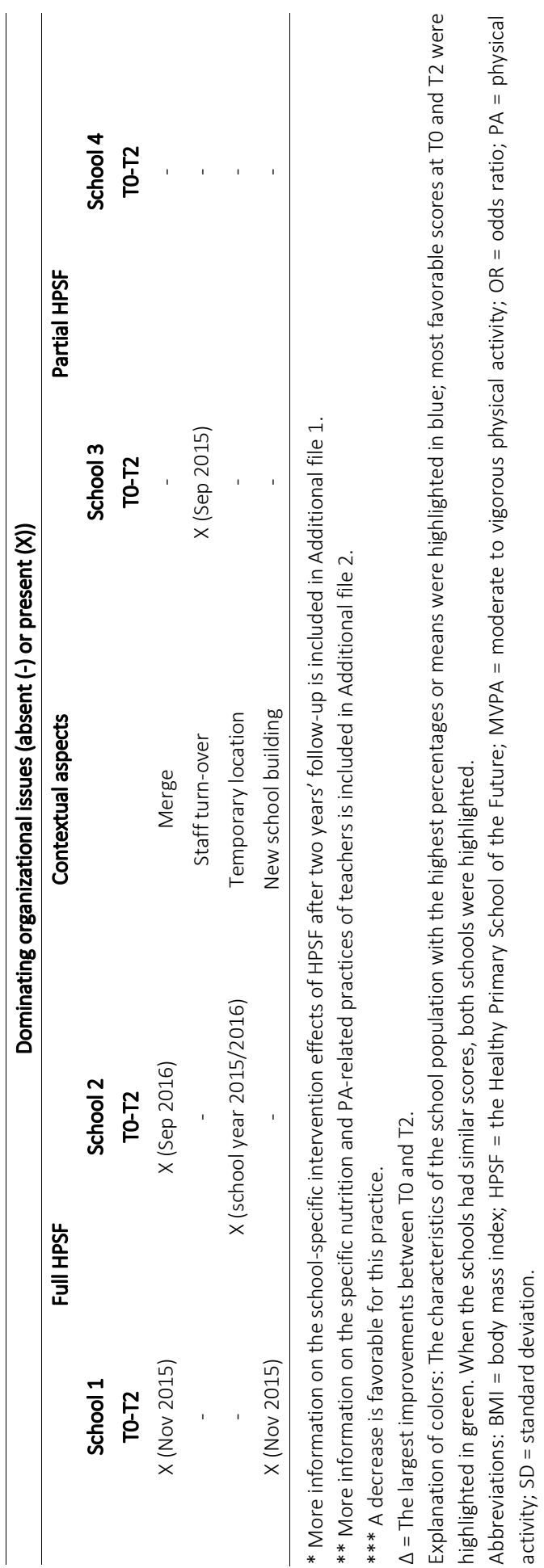




\section{Discussion}

The current study assessed and compared the contexts and effects of HPSF in four schools and explored whether aspects in the context relate to larger favorable effects. The results showed that the four school contexts were different at the start of the HPSF, and that they evolved differently during the two years of HPSF, and that the effects of HPSF were different for the four schools. These findings underline our rationale that the school context influences the effects of school health promotion efforts. These varying effects across schools can be seen as the result of the nonlinearity of the system and the interaction of contextual aspects with the HP changes in the school. Since each complex adaptive school system is unique and can react in a different way, varying effects can be expected and seem to represent the natural variation within complex adaptive systems [159].

The findings in the current study showed that potentially moderating contextual aspects were found on the level of the children, the employees, and the school itself. These levels were not separated in a complex adaptive school system but they also continuously interact with each other. It is challenging and maybe even impossible to fully understand this complex systems behavior and its impact on the effects on children's health and health behaviors. In this study, however, we aimed to take a first step towards this understanding by exploring the contextual aspects in the schools that appeared to be related to larger effects.

Concerning the level of the children, we found that larger effects were observed in the schools in which a smaller percentage of children were part of the lowest SES group, and in which children had the most favorable starting position regarding their health behaviors. Even though, on average, the effects were favorable for all schools and the focus was already on a low SES area, the findings regarding SES seemed to indicate that HPSF is more favorable for the higher SES groups. This suggests that HPSF might still contribute to the socioeconomic health inequity gap [120]. The moderation effect of SES indicates that the intervention outcomes interact with the children's background in the home context. It underlines that the school system is an open system, and that effects of HP changes in the school can also be moderated by aspects in the home context or neighborhood [16, 17]. Moreover, the findings showed larger effects of HPSF not only in the higher SES schools, but also in the schools in which children had the most favorable starting position regarding their health behaviors. The association between these two aspects was investigated in-depth for the children in the HPSF schools by Vermeiren et al. [160], and they were in line with other studies which showed that less favorable health behaviors tended to be associated with a lower SES [161]. This means that a school that includes more children with a lower SES background may also have more children with less favorable health behaviors, and vice versa. This seems to indicate that the moderation of these two child characteristics is 
clustered. Overall, the findings suggest that even though HPSF was beneficial in all schools, it may lead to smaller effects in the schools that included the most disadvantaged group of children. It should be examined whether further adaptation of the HPSF to the school's population is needed or whether these schools just need more time to achieve more favorable effects.

Concerning the level of the employees or intermediaries, we found larger effects in the schools with the most improvements in HP practices of the teachers (specifically related to modelling and encouragement of healthy nutrition and PA) and schools with the least barriers perceived by the external pedagogical employees. The moderating role of teachers' HP practices was in line with the study by Gubbels et al., who investigated this in the childcare setting. They showed the importance of favorable food practices of employees at the childcare organizations, such as modelling behavior, for a healthy food intake of the children [102]. The findings of the current study suggested that by improving the HP practices of teachers, the larger effects of HP changes in the school can be achieved. Thus, it is recommended to directly intervene in these HP practices of teachers. The findings also showed that a focus on the perception of external pedagogical employees, the main implementers of the HP changes, can optimize the effects. These external employees were provided by childcare organizations and were employed to avoid increasing the teachers' workload even further. This integration of the childcare organization during school hours was not intended to provide a temporary solution, but to provide professional employees for the implementation of the HP changes, and to change the school's organization in a sustainable way. The findings in this study imply that to achieve larger effects, it is recommended to monitor the perception of these main implementers regularly to provide input for feedback loops. These feedback loops, also visualized in the program theory (Figure 1), should make it possible to understand and tackle perceived barriers. Overall, these findings on the level of the employees suggest that directly investing and intervening in them by improving teachers' HP practices and monitoring, as well as tackling the barriers perceived by the main implementers, may contribute to achieving larger effects of a health promoting school initiative.

Regarding the level of the school itself, we found that larger effects were observed in schools when they were able to synergize existing organizational issues in the school with the HPSF. Some schools had to deal with a dominating organizational issue, e.g., merger process, that disrupted their normal functioning. Such a disrupting event in the school can create an opportunity for HPSF, i.e., the merger process can build up momentum for a new start, which helps to create a new way of working in which HPSF is also included. Therefore, it is recommended to gain insight into whether organizational issues exist in the school and how this can be used as an opportunity to build up momentum for HPSF. 
The findings in this study demonstrate that a broad insight into the school context is crucial for understanding the intervention effects of HP changes in a complex adaptive school system. The focus should not merely be on intervention evaluation, but also on the context evaluation $[42,43,54]$. The findings suggest that it may result in an over-or under-estimation of the effects of HP changes when different school contexts are combined in the analyses. Therefore, it is recommended to also examine the effects separately for each school context. Moreover, the results of this study suggest that the average effect sizes of intervention outcomes do not provide a full answer regarding the effectiveness $[159,162]$. Larger effects may be achieved due to the interaction with specific contextual aspects, e.g., more children in the school with a higher socioeconomic background or organizational issues in the school. Therefore, when evaluating the effectiveness of HP changes, the focus should not only be on the effect sizes and outcomes, but also on aspects in the context that interacted with the HP changes. This context-oriented evaluation of HP changes contributes to a better understanding of the moderating role of the school context on the effects of HP initiatives. It may explain the variation in effects across schools, and it can provide insight on which contextual aspects to focus on or intervene in to optimize the effects.

\section{Strengths and limitations}

Several strengths and limitations of the study should be considered. Since HPSF was quite comparable between S1 and S2, and between S3 and S4, we saw an opportunity to explore the moderating role of the school context on the intervention effects of the HPSF. However, a limitation is that we could not determine whether differences in effects between schools were due to differences in the implementation of the HPSF [20, $30,88]$. Furthermore, two comparisons are still limited; however, we were able to combine the results of both comparisons and form stronger conclusions about the moderating role of the school context. Future research should investigate whether the findings of this study also apply to other schools. Finally, assessing the school contexts had several limitations. We examined many of the contextual aspects in a quantitative manner, which may not fully capture each aspect. It was also impossible to fully assess and understand all aspects of each school's context citing limitations in resources, time, and participant burden [47]. This might have led to missing important, possibly moderating, contextual aspects. Nevertheless, we were able to examine contextual aspects on all levels in the school and to focus on the aspects suggested by other researchers as relevant for improving school health promotion [21, 42, 68, 71]. 


\section{Conclusion}

Similar HP changes lead to different outcomes across schools due to differences in the school context. Potentially moderating contextual aspects in the Healthy Primary School of the Future were found at the level of the children, the employees, and the school itself. When evaluating the effectiveness of HP changes, the focus should not only be on overall effect sizes, but also on which aspects in the context interacted with the HP changes. The adoption of a complex adaptive systems perspective contributes to a better understanding of the variation in effects across schools and it can provide insight on which contextual aspects to focus on or intervene in to optimize the effects of HP initiatives. 


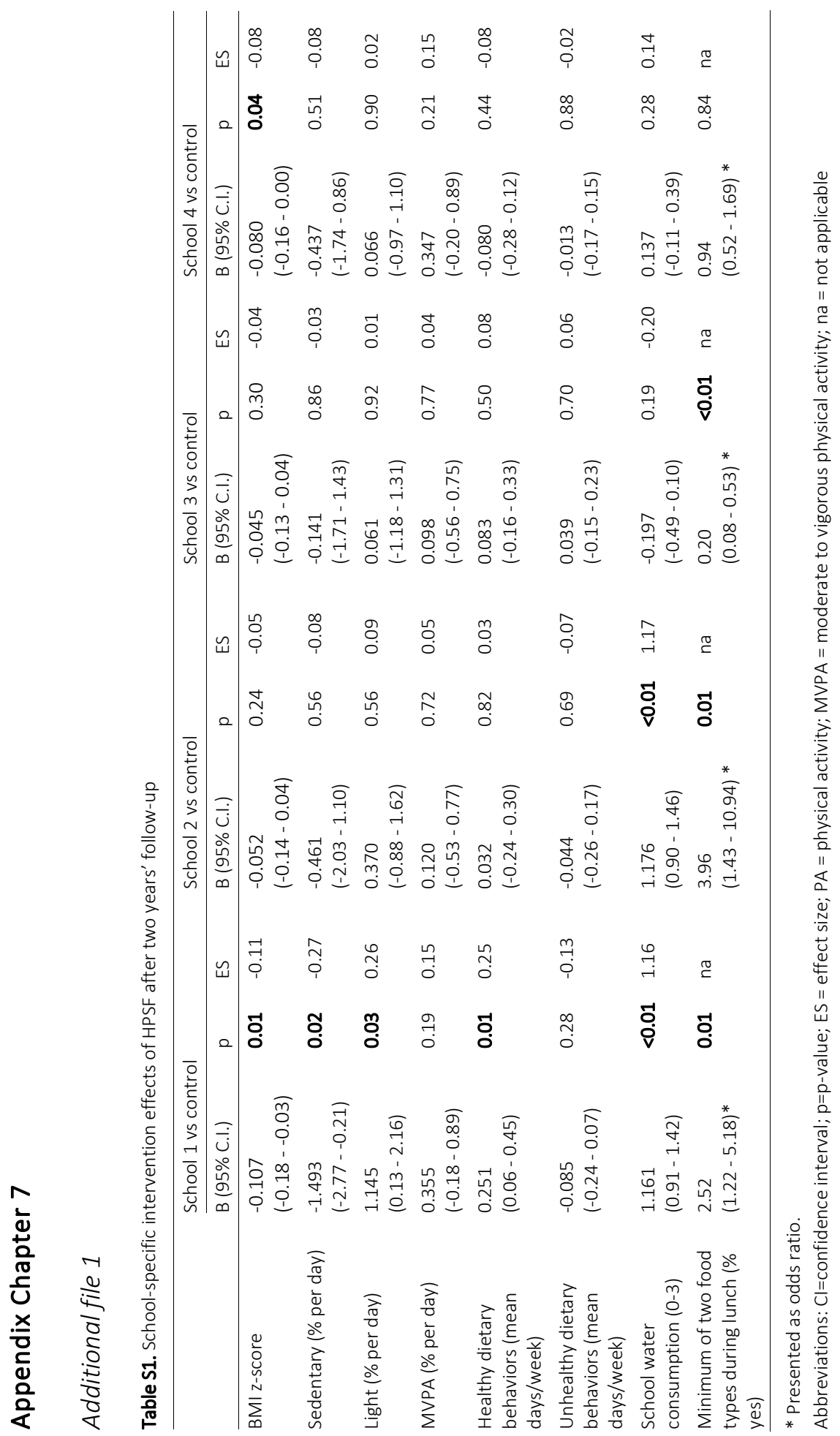




\section{Additional file 2}

Table S2a. Explanation of the nutrition-related practices of teachers

\begin{tabular}{|c|c|}
\hline Healthy modelling & $\begin{array}{l}\text { I consciously eat healthy food products when the children are around. } \\
\text { For example, by eating fruit when the children are around. }\end{array}$ \\
\hline $\begin{array}{l}\text { Prevent unhealthy } \\
\text { modelling }\end{array}$ & $\begin{array}{l}\text { I consciously do not eat unhealthy food products when the children are around. } \\
\text { For example, by eating unhealthy food products in the teachers' room or at home. }\end{array}$ \\
\hline Encouragement & $\begin{array}{l}\text { I encourage the children to eat healthily. } \\
\text { For example, by encouraging the children to bring and eat vegetables as a } \\
\text { midmorning break. Or by encouraging them to first eat their lunch and afterwards } \\
\text { their sweet snack. }\end{array}$ \\
\hline Involving & $\begin{array}{l}\text { I involve the children in things that concern a healthy diet. } \\
\text { For example, by involving the children when handing out fruit. By involving children } \\
\text { in a vegetable garden. By letting children taste different sorts of fruit during the } \\
\text { lesson. }\end{array}$ \\
\hline Discussing & $\begin{array}{l}\text { I discuss nutrition with the children. } \\
\text { For example, by discussing the food products they eat and like. }\end{array}$ \\
\hline Educating & $\begin{array}{l}\text { I teach the children about nutrition. } \\
\text { For example, by teaching the children where food comes from. By explaining that } \\
\text { eating healthy foods is better than candy or cookies. }\end{array}$ \\
\hline Providing feedback & $\begin{array}{l}\text { I give feedback to the children concerning their dietary behavior. } \\
\text { For example, by commenting when a child often does not finish his/her lunch, often } \\
\text { eats unhealthy foods or does not take the time to eat. }\end{array}$ \\
\hline Visibility & $\begin{array}{l}\text { I ensure that healthy food products are visible for the children (e.g. pictures). } \\
\text { For example, by hanging up pictures of fruit in the classroom. Or by hanging up } \\
\text { visualizations (e.g. mind map) of group sessions about healthy foods. }\end{array}$ \\
\hline Routines & $\begin{array}{l}\text { I ensure that there are healthy habits during moments of eating and drinking in } \\
\text { school. } \\
\text { For example, by taking enough time for lunch and to wait till everyone is finished. }\end{array}$ \\
\hline Monitoring & $\begin{array}{l}\text { I try to watch what the children are eating during the day. } \\
\text { For example, by paying extra attention when a child often eats unhealthy foods as } \\
\text { midmorning break or lunch. }\end{array}$ \\
\hline Rules & $\begin{array}{l}\text { I strictly follow school policy on nutrition in school and in class. } \\
\text { For example, by following strictly the policy for celebration treats or the prohibition } \\
\text { on energy drinks. }\end{array}$ \\
\hline Pressure to eat & $\begin{array}{l}\text { I insist that children finish their meal. } \\
\text { For example, by insisting that children completely finish their lunch. }\end{array}$ \\
\hline Instrumental feeding & $\begin{array}{l}\text { I reward the children sometimes with unhealthy food products when they did } \\
\text { something well. } \\
\text { For example, by rewarding the children with candy in a certain lesson when they did } \\
\text { something really well. }\end{array}$ \\
\hline
\end{tabular}


Table S2b. Explanation of the PA-related practices of teachers

\begin{tabular}{ll}
\hline Encouragement & I encourage the children to be physically active. \\
& For example, by discussing the advantages of physical activity. By providing \\
& ideas for PA games during breaks and playing these games during PE. \\
I reward the children for being physically active. \\
For example, by giving the children compliments when they are very physically \\
active during breaks or performed well during PE. \\
I involve the children in things that concern physical activity. \\
For example, by giving the children a choice in the kind of energizer for that \\
moment, or the kind of activity during part of the PE.
\end{tabular}

Healthy modelling

Discussing

Educating

Discouragement

Providing feedback

Availability

Accessibility

Routines

Warning PA

Monitoring

Rules

Pressure to be physically active

Instrumental feeding
I am consciously physically active when the children are around.

For example, by playing outside with the children during breaks, dancing together with the children, or do an energizer together. By commuting to school also in an active way.

I discuss physical activity with the children.

For example, by discussing the different possibilities for physical activity that the children like or dislike. By asking which sports they play in their leisure time.

I teach the children about physical activity.

For example, by teaching the children new options to be physically active or teaching them the rules of games. By teaching the children that physical activity is good for your health.

I correct children when they do not sit quietly during lessons.

For example, when a child is moving on his chair continuously.

I give feedback to the children about their physical activity behavior.

For example, by discussing their physical activity behavior and giving ideas for more ways of being physically active.

I ensure there is PA-friendly equipment available for the children.

For example, by preparing outdoor toys like a skipping rope or football.

I enable the children to be physically active.

For example, by regularly using energizers in the classroom. By always giving children the opportunity to be physically active even when it is raining outside.

I ensure healthy PA habits in school.

For example, by planning the times for energizers. By ensuring that the PA activity is fun and that everybody can join in.

I warn the children about the possible risks of physical activity and playing outside.

For example, by warning the children they can hurt themselves or get dirty when playing outside.

I check in general the amount of physical activity of the children during a day.

For example, by paying extra attention to someone who is not often physically active during breaks or PE.

I strictly follow the school policy on physical activity in the school and in the classroom.

For example, by strictly following the rules in the classroom or school yard regarding physical activity.

I insist that the children be physically active.

For example, by insisting that children always go outside during breaks, or that children always participate during PE or energizers.

I reward the children sometimes by watching a movie together or letting them be on the computer when they did well.

For example, when it is almost holiday and they worked well in the last weeks. 



\section{Chapter 8}

General discussion

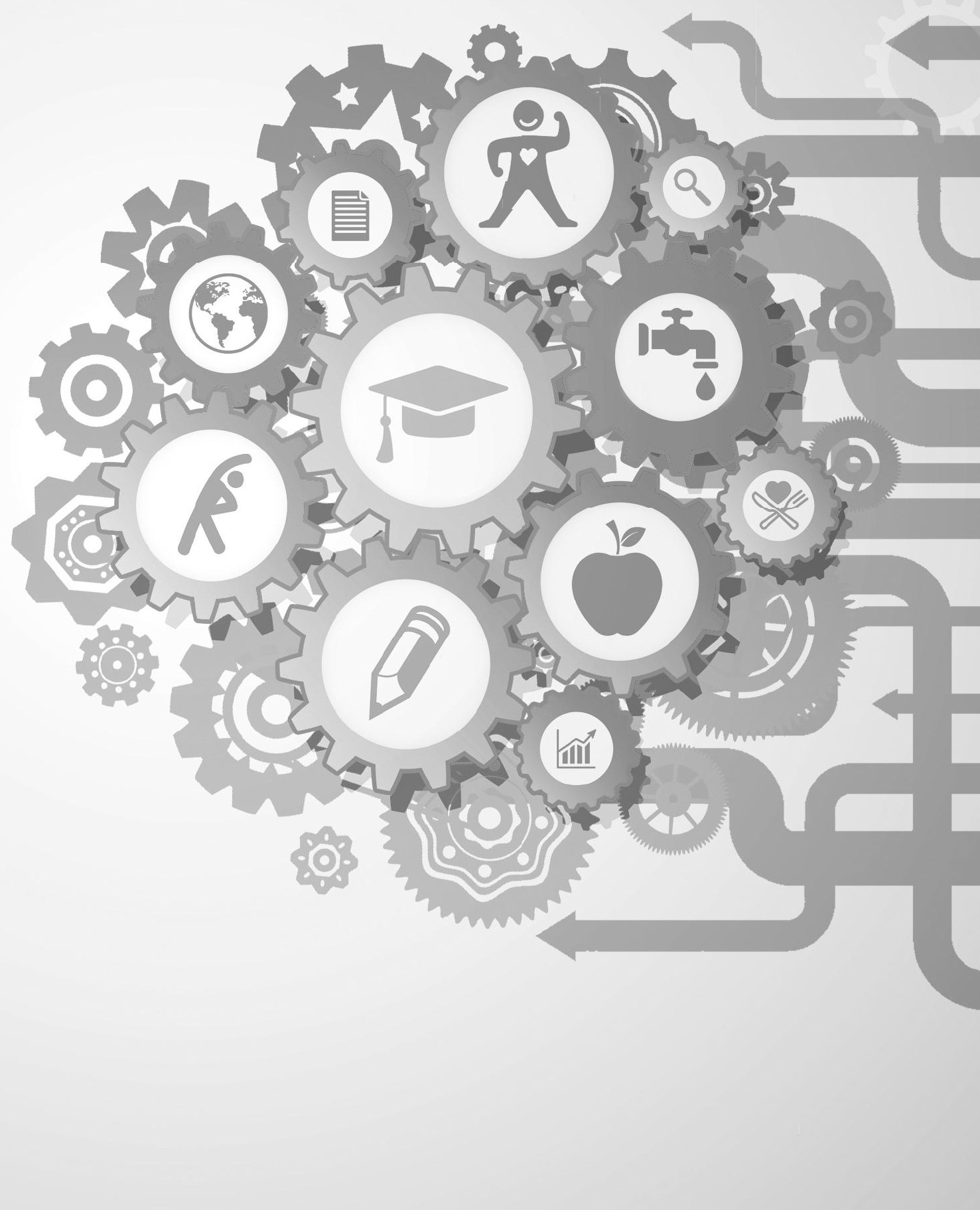


The research reported in this dissertation aimed to evaluate the Healthy Primary School of the Future (HPSF) in four complex adaptive school systems. HPSF is a Dutch initiative based on the principles of the Health Promoting School framework that aims to sustainably integrate health and well-being within the whole school system. The decision to adopt the theoretical perspective of considering schools as complex adaptive systems has led to the development of HPSF and the programme theory. This programme theory (Figure 1) visualizes the hypothesized process of how HPSF integrates into the school context. It acted as a conceptual basis for the overall contextual action-oriented research approach (CARA) and led to the formulation of the main five research questions of this dissertation:

1. How was HPSF developed and implemented and how did it interact with the context of the four schools?

2. What was the effect of HPSF on children's BMI z-scores and their dietary and PA behaviours after one and two years?

3. What was the effect of HPSF on children's dietary and PA behaviours at school and at home?

4. To what extent did HPSF have different effects within specific subgroups of children?

5. What was the moderating role of the school context on the effects of HPSF?

This chapter discusses the most important findings from the reported studies. It is divided into three sections: 1) the implementation of HPSF, 2) the effects of HPSF, and 3) the research approach. To improve readability, each section starts with a short summary about its topic. The first section reflects on the strategies of HPSF to integrate health promotion in school in interaction with its school context. This relates to the first research question, the left part of the programme theory. The next section discusses the effects of HPSF on children's BMI z-scores and their dietary and PA behaviours, and the moderating role of the school context. This relates to research questions two to five, the right part of the programme theory. The last section elaborates on the overall research approach of the different studies, i.e., CARA, by discussing the gained experiences and by providing guiding principles. Finally, all findings and experiences are used to reflect on the theoretical perspective of considering schools as complex adaptive systems and to provide an overall conclusion regarding both the effects and strategies of HPSF as well as the application of CARA. 


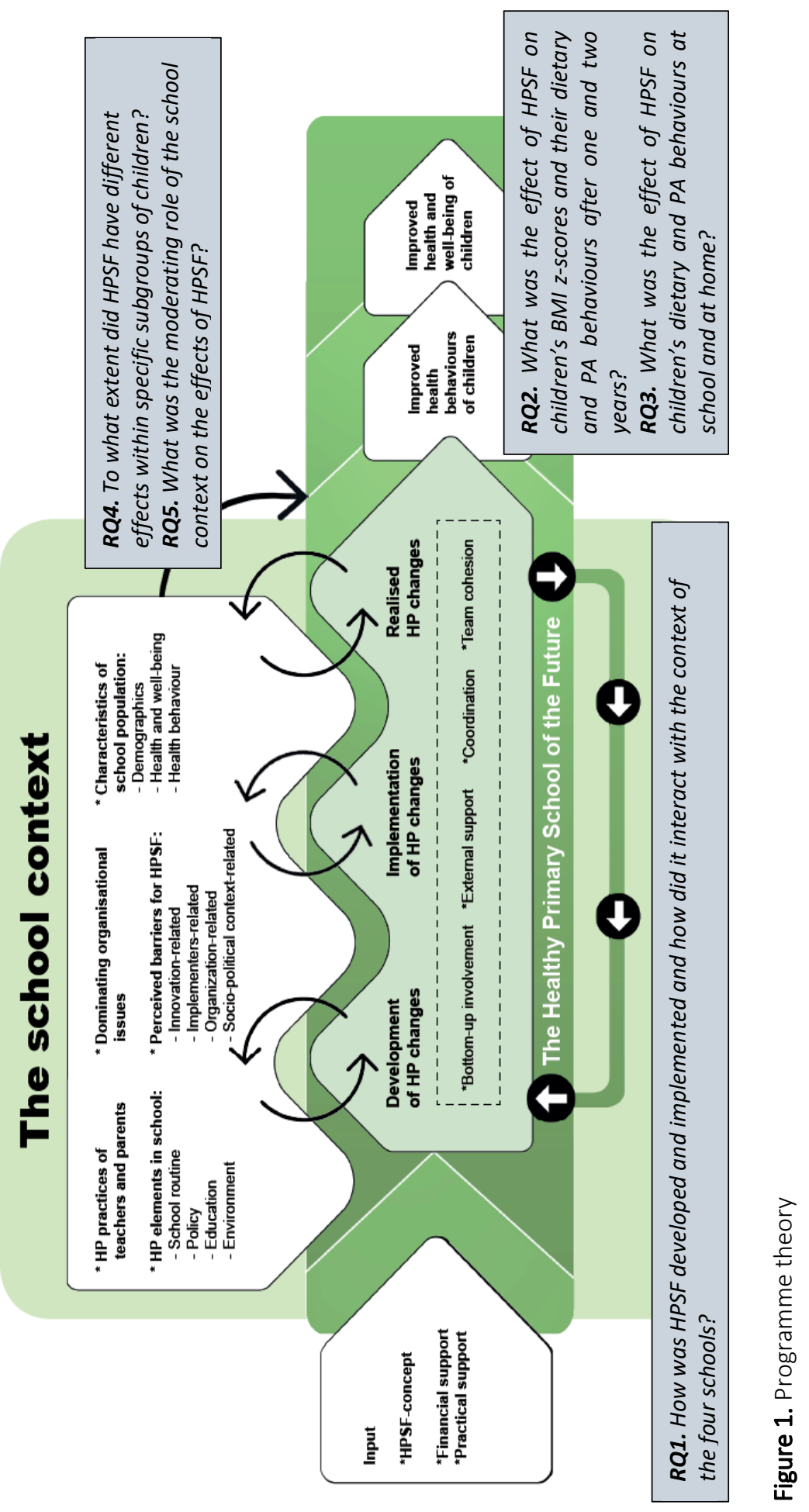




\section{Implementation of HPSF and its interaction with the school context}

HPSF intends to establish a broad collaboration between school, parents, and external partners, which should lead to a co-creation movement in schools consisting of topdown and bottom-up processes. HPSF also intends to create some form of positive disruption in the schools by initiating two health-promoting (HP) changes top-down: 1) a free healthy lunch each day and 2) daily structured physical activity (PA) and cultural sessions, both led by external pedagogical employees provided by childcare organizations. These two changes are hypothesized to create momentum for bottomup processes to implement additional HP changes. It was also hypothesized that it was essential for the implementation of HPSF that all HP changes are adapted to the context and vice versa, i.e., mutual adaptation. Research question one was formulated to investigate these hypotheses.

\section{Main findings}

The schools had to deal with different contextual issues and they could decide themselves on the adoption and implementation of HP changes. This resulted in differences between the schools regarding HPSF. Two of the four intervention schools decided to implement both top-down changes (full HPSF), i.e., the lunch and the structured PA and cultural sessions. To realize these changes during the lunch break, both schools extended the lunch break time to about 60 minutes. Therefore, children attended school until approximately 15:30/15:45 instead of 15:00. Both schools implemented several additional HP changes: they both provided water bottles to all children, improved their school's health policy, and introduced an educational lunch. The other two intervention schools decided to implement only the structured PA and cultural sessions each day (partial HPSF). They did not provide a healthy lunch nor did they increase their lunch break time or implement additional HP changes. The implementation of the provided lunch changed the dynamics in the full HPSF schools, which created momentum for more bottom-up processes, including more involvement and support of teachers and parents. Most of the additional HP changes were described as being facilitated by this lunch. The partial HPSF did not implement the lunch and a limited transformation was found in these schools. It was perceived as essential in all participating schools to have bottom-up involvement throughout the process of change to contextualize and optimize the HP changes and to create ownership. Combining this bottom-up involvement with top-down advice, and external practical support helped the schools to successfully implement the HP changes in their school context. Sufficient coordination and communication at the school level, the availability of external pedagogical employees, team cohesion, and feedback loops among all actors involved enhanced the implementation of the HP changes (Chapter 3 ). 
Main differences in implementation between the partial and full HPSF: Wholesystem change

In all participating schools, the greatest resistance was found among teachers and parents regarding the provision of a healthy school lunch. As a result, two schools did not adopt this lunch and focused on the PA and cultural sessions. Even though these sessions were also major changes in the schools, they mainly impacted children during lunch break time and did not lead to a disruption of the system. The two full HPSF schools did implement the lunch. As a consequence of this implemented lunch, the way of working in the schools was disrupted, and the schools had to adapt to this disruption. This disrupted situation has led to the development of many interactions and feedback loops between different components of the school system: the full HPSF schools had to find a new state of stability. Findings showed that this new stability in the system has resulted in an integration of the HP changes in the school and more focus on health and wellbeing. These transformations in the two full HPSF schools indicate that it is possible to successfully change the whole school system.

\section{Successful strategies to implement HPSF}

Both the provision of the lunch and the PA and cultural sessions aimed to create a positive disruption in the school. Even though the results showed that only the lunch succeeded in creating this disruption and did act as a catalyst for wider school health promotion, both HP changes were major changes for the schools and were not easy to achieve. All people involved had to embrace the idea that to have a real, positive impact on the health and wellbeing of children, something significant had to change in the school system. To this purpose, the schools put a lot of effort into getting the support of teachers and parents for the HP changes and collaborated with many external partners to make it possible to realize such significant changes. One of the most important external partners were the childcare organizations. By deploying external pedagogical employees, provided by the childcare organization, it was possible to realize the changes without increasing the workload of teachers even further. This integration of the childcare organization during school hours was not meant to be a temporary solution, but to provide professional employees for the implementation of the HP changes and to change the school's organization in a sustainable way, which should create a more integrated school day for children. Moreover, realizing both HP changes in the full HPSF schools was only possible when the school day was extended. Implementing the HP changes meant that the school day in both schools now finished at 15:30/15:45 pm instead of 15:00 pm. This is against the general trend in the Netherlands of shortening the school day as much as possible. Implementation of the HP changes also meant that the people in the school first had to adopt the concept of the changes and then the changes had to be adapted to fit the school context. This adaptation process included many feedback loops among the different components of 
the school system and continuous trial and error. This was needed to fit the HP changes to the school context, such as the characteristics of the children in the school and the different aspects of the environment inside and outside the school, i.e., the social, political, economic, and physical environment. For example, the feedback loops between the people in the school and the project team led to many adaptations in the content of the lunch. This was needed to make the lunch more recognizable for the school children and to offer products that parents can buy in a low-budget supermarket. Overall, the implementation of HPSF in the schools was possible due to the combination of these different strategies, i.e., the positive disruption, shared vision, external pedagogical employees, extended school day, and the feedback loops. As a consequence of its implementation, many children, teachers, and parents have perceived the benefits of a school that integrates health and education and indicated that they did not want to go back to the old situation anymore and lose these changes.

The described strategies can help to disseminate HPSF to other primary schools. In addition to these strategies, it should be noticed that HPSF had a luxury position due to the large financial support from the Province of Limburg. This is an exceptional situation as often only limited funding is available for school health promotion initiatives. The financial support enabled the implementation of the major HP changes in the participating schools. To disseminate HPSF or its components to other primary schools, solutions must be found for the high costs of HPSF. Moreover, in other Dutch primary schools, the positive disruption may be created by the implementation of a healthy school lunch, as that is not typical practice in the Netherlands. In other countries, e.g., the UK or Belgium, the implementation of a healthy lunch may not create this positive disruption as the schools in these countries already provide school lunches each day. Differences in the school context may thus require different HP changes.

\section{The interaction between HPSF and the school context}

The main findings revealed the interaction between HPSF and the school context, which indicates that integrating health promotion in a school system is not a linear trajectory with a beginning and an end, but rather a non-linear, complex and dynamic process. This demonstrates that a standardized program package of HPSF with a form that looks the same in each school would not have worked. Until recently, many claimed that all forms of adaptation indicate a lack of fidelity [88, 163, 164]. Fidelity means implementing an intervention in the exact way its developers initially designed it to be implemented and staying true to the evidence base: a lack of fidelity is thus perceived as a threat to intervention effectiveness [88, 163, 164]. Although the importance of implementation fidelity is recognized, more flexibility could create a better fit of HP changes within a specific school context, as the needs, wishes, and opportunities of the context in which the changes are implemented could be addressed [53]. This adaptation may increase the likelihood that the implemented HP changes will result in sustainable 
effects [88]. Changing the focus of fidelity from the form of an intervention to its function provided a solution for this fidelity issue in complex adaptive systems $[43,67]$. By focusing on the function of HPSF and not its form, it allowed for adaptions to create a better fit with each school context. For example, HPSF includes PA sessions after lunch to improve children's PA behaviours (function), in which each school developed their own format (form) for these sessions, e.g., structure of the sessions, where they take place, which PA activities are used, whether children can choose between activities, etc. A continuous process of trial and error, feedback loops, and communication between all people involved helped to create a form that fitted the school context. This co-creation included continuous interactions between bottom-up involvement, top-down advice and external practical support. The interactions between these three were perceived as crucial for the integration of HPSF in the schools, as together they include all the required knowledge, experiences, and resources. Bottom-up involvement was needed as teachers, children, and their parents knew best which HP changes were most appropriate in their school, and it helped to create ownership and school-wide support. Top-down advice from experts was needed since they possess the knowledge from previous research about evidence-based HP changes or conditions and how to integrate health promotion in schools. This did not mean that they insisted on what should happen, but that they shared their knowledge and experience to support the schools. Finally, external partners provided the essential resources and practical support that was needed to realize the HP changes. They were able to provide support in terms of personnel, money, and materials, but also had specific practical knowledge and experience to share. This combination of bottom-up involvement, top-down advice, and external practical support can be seen as an example of 'navigating in the middle' [48]. It shows how these top-down and bottom-up forces meet, with the local context being respected whilst making use of the knowledge and support of the broader system.

\section{The effects of HPSF and the moderation of the school context}

HPSF aims to implement HP changes in all aspects of the school system. It is hypothesized that all these HP changes combined favourably affect the health behaviours of the school children, which should lead to improved health and a healthier weight status of children. It is also hypothesized that even when schools implement similar changes, their impact may differ by school, due to the moderating role of the school context. Research questions two to five were formulated to investigate these hypotheses. 


\section{Main findings}

Significant favourable intervention effects on children's dietary and PA behaviours were found for the full HPSF for, among others, school water consumption, lunch intake of vegetables and dairy products, sedentary time and light PA. In contrast, almost no significant favourable results were found in the partial HPSF (Chapter 5). In addition, a significant intervention effect on children's Body Mass Index (BMI) z-score was found, with z-scores being calculated using Dutch reference values [5]. Children's BMI z-scores in both the full and the partial HPSF significantly decreased after two years' follow-up compared with those of children in the control schools, whose mean BMI z-score actually increased from baseline to two years. This favourable effect was already significant after one year's follow-up in the partial HPSF, but not yet in the full HPSF (Chapter 4). When dividing children's health behaviours into behaviours at school and at home, it was found that children's PA behaviours at school had increased in both the full and partial HPSF. Children's dietary behaviours in school improved only in the full HPSF. At home, children in the full HPSF did not compensate for the HP school activities in their PA or dietary behaviours. In the partial HPSF, the children became less active at home (Chapter 6). None of the potential effect modifiers (gender, baseline study year, socioeconomic status (SES), and baseline weight status) significantly moderated the effects on children's BMI z-score (Chapter 4). Regarding the effects on children's dietary and PA behaviours, it was found that the effects in both the full and partial HPSF were less favourable at school for younger children and less favourable at home for children with lower SES scores. In general, the effects of the full HPSF were more equally beneficial for all children than the partial HPSF (Chapter 6). A moderating role of the school context on the effects of HPSF was observed. Larger effects were found in the schools in which a smaller percentage of children were part of the lowest SES group, and in which children had the most favourable starting position regarding their health behaviours. Larger effects were also found in the schools with the most improvements in HP practices of teachers and with the fewest perceived barriers of the external PE. Finally, larger effects were observed when schools were able to synergize HPSF with existing organizational issues in their school (Chapter 7).

\section{Main differences between the effects of the full and partial HPSF: Synergy}

The findings showed that, compared to the partial HPSF, the full HPSF was more effective in improving children's health and health behaviours, had more equally beneficial effects for all children, and succeeded better in creating a transferring effect to the home context. These findings suggest that a bigger impact is created in the full HPSF due to the disruptive effect of the lunch and its side effects in terms of an increased willingness to adopt and adapt to several additional HP changes. Moreover, the full HPSF also focused on both dietary and PA behaviours, while the partial HPSF mainly focused on children's PA behaviours. The synergy that occurred between the 
two health behaviours in the full HPSF may also explain the more favourable effects, because the probability of enhancing one health behaviour increases when an individual has successfully changed the other health behaviour $[125,126]$. This means that, for example, an increase in PA may lead to improved dietary behaviours and vice versa. Simultaneously addressing two clustered health behaviours in school health promotion may thus be more effective due to the facilitation of this synergistic effect.

\section{Clinical relevance of the effects of HPSF on children's health and health behaviours}

The largest effects of HPSF regarding children's health behaviours were found in the children's water consumption at school and their lunch intake. Smaller effects were found in children's PA behaviours and their overall dietary behaviours. The combination of smaller and larger improvements in different kinds of health behaviours of children led after two years' follow-up to a small favourable effect on children's health in both the full and partial HPSF, which is indicated by their BMI z-score. These small intervention effects on BMI z-score are promising for three reasons: 1) they are already visible after two years of implementation, 2) they indicate a positive change compared to the increasing BMI trend observed in the control schools, and 3) they are slightly higher than the effect sizes found in several meta-analyses regarding school-based interventions $[108,109,117]$. Even though this favourable effect on children's BMI zscore seems promising, the effect can be indicated as a small effect, according to Lipsey's guidelines [100], and therefore raises the question regarding its clinical relevance. Previous studies have explored the minimal differences in BMI-z scores in overweight children to ensure clinically relevant health benefits [101]. As yet, little is known about when population-level public health interventions achieve clinical relevance [165]. In general, the effects need to be sustained to obtain clinical relevance, which implies that to draw a better conclusion about the clinical relevance of HPSF, the long-term effects should be investigated. This is included in the overall study, in which the effects of HPSF are being examined for another two years of implementation. Prediction models are also included in the overall study to predict the effects of HPSF when the children become adults. This prediction modeling can contribute to providing more insight into the clinical relevance of the effects of HPSF.

Interactions with other microsystems: For whom and where is HPSF most effective?

To realize the sustained effects of HPSF, new habits and routines need to develop in children's health behaviours, which requires a shift in the social norms of all people in the school regarding 'normal' health behaviours [166]. This is challenging to achieve since the school is an open system, which means that changes in the school interact with other microsystems of a child, such as their home context or neighbourhood. The 
main findings showed this interaction: less favourable effects were found at home for children with a lower socioeconomic background. By including the home context in the school's HP changes, e.g., homework assignments that include the parents and/or by focusing directly on health promotion in the home context, children should be enabled to make healthier choices both at school and at home [151]. In this way, more consistency is created between a child's different microsystems, particularly for children with lower socioeconomic backgrounds. The challenge of creating sustainable effects reaches further, because primary school is only one of a child's microsystem from the age of 4-12: after the age of 12, children will enrol in a secondary school. To sustain the effects of HPSF, secondary schools should also enable children to make healthier choices. To examine the sustainability of the effects of HPSF, it is recommended to keep track of the children who took part in HPSF schools after they finish primary school. The regular monitoring of secondary school children by the regional Public Health Services may be a helpful way to accomplish this follow-up examination.

\section{The moderating role of the school context on the effects of HPSF}

The main findings regarding the moderating role of the school context showed that even when schools implemented similar HP changes, this did not lead to similar effects. This demonstrates that studying the effects of HPSF cannot be done in isolation from its context, which is in line with the complex adaptive systems perspective and the recommendations of other researchers $[42,43,54]$. Moreover, since contextual aspects on different levels of the school system seem to have a moderating role on the effects, the findings underline that a broad understanding of the school context is crucial for a proper understanding of the effects. This moderating role of the context suggests that when analyzing the effects of changes in a complex adaptive system, they need to be examined separately for each context to prevent over- or underestimation. The findings also suggest that the average effect sizes of outcomes do not provide a full answer regarding the effectiveness of HP changes $[159,162]$. Larger effects are also achieved due to the interaction with specific contextual aspects. Therefore, when evaluating the effectiveness of HP changes, the focus should not only be on the effect sizes and outcomes, but also on aspects in the context that interacted with the HP changes. This context-oriented evaluation may contribute to explain the variation in effects across schools and can provide insight on which contextual aspects to focus on or intervene in to optimize the effects. Finally, the moderating role of the context shows that transferring HPSF or components of HPSF to another school does not automatically lead to similar effects. A proper understanding of the school context is thus needed to both implement and evaluate HPSF in a specific school. 


\section{The overall research approach: CARA}

To align our research of HPSF to the complex and adaptive nature of the school systems, we translated the principles of action research into a contextual actionoriented research approach (CARA). The purpose of CARA for researchers is to contribute to a health promoting school initiative and to conduct a thorough evaluation at the same time. Basic properties of CARA are its specific focus on contextual differences and the use of monitoring and feedback to both support and evaluate the process of change. The approach centers around four key questions: 1) What is the pre-existing context of each school? 2) How does the process of change in each school evolve and which factors affect this process? 3) How can research contribute to the process of change? and 4) Do children's health and health behaviours improve as a result of the HP changes? (Chapter 2).

Experiences of evaluating change in a complex adaptive system

Based on my experiences with CARA generated by the research, I have described as concretely as possible, how my co-researchers and I have dealt with the main challenges of conducting research in complex adaptive school systems (Table 1). In addition to this table, I elaborate on the experiences with CARA regarding specific parts of the research. 
Table 1. Evaluating change in complex adaptive systems: Challenges and our solutions

Challenges to evaluate change in Our solutions based on CARA

complex adaptive systems

How to be sensitive to the dynamics of the local context?

How to be flexible to deal with the unpredictability of the system?

How to analyze the evolution of a complex adaptive system?

How to evaluate several different contexts and draw overarching conclusions without losing sight of each unique context?

How to remain objective for the scientific evaluations but also be involved in and give support to the process of change?

\begin{abstract}
We (the researchers) continuously monitored and followed the things that were happening in the schools by regularly collecting a wide range of data in the school systems. We used different methods to combine the accuracy of quantitative methods, e.g., questionnaires, and the in-depth insights of qualitative methods, e.g., interviews or observations. This provided us with a broad understanding of the school systems. We also documented the smaller and larger events that occurred in the schools.
\end{abstract}

We used hypotheses to determine what to measure, but we adapted throughout the process to be able to measure the unintended effects as well. This also means that we had to make decisions regarding appropriate methods along the way instead of only preparing a research proposal beforehand to be able to react on these unexpected changes or effects. In general, the most appropriate methods were ones that were feasible for us as researchers and the study population, and were quickly processed and analysed to provide real-time feedback.

We aimed to capture the events that occurred in the system by organizing the collected data chronologically. This enabled us to show the link between events that represent the process of change in the context.

We investigated the process of change in four different contexts, with each context being treated as a unique case. Similarities and differences between these four contexts were studied. The effects were examined by first conducting overall analyses, which was followed by quantitative analyses of effect modifiers and qualitative comparisons to study the moderating role of the context.

We had to be close enough to the practice to know and understand what was happening, but also be distant enough to evaluate the bigger picture. By conducting the process evaluation prior to the effect evaluation, we were involved in the process of change without knowing the effects. In this way, the process and effect evaluation became complementary and resulted in more complete findings. Using the principle of data triangulation for the process evaluation and a quasiexperimental design for the effect evaluation helped us to study the process and effects as objectively as possible.

\section{Study design}

It was not perceived as desirable nor feasible to randomize a population-level intervention, such as HPSF, as it attempts to factor out the system's context $[49,167]$. Therefore, a quasi-experimental study design was used to evaluate the effects of HPSF on children's BMI z-scores and health behaviours. The design enabled us to examine the effects between the three school groups over time and at the same time to enrol schools on the basis of their motivation. We did not include any randomization, which 
has resulted in significant baseline differences, with higher BMI z-scores and unhealthier dietary and PA behaviours found in the children in the control schools. These baseline differences seem to indicate that the children in the control schools have developed stronger unhealthy habits, which have already led to greater overweight or obesity. These stronger habits can be more difficult to change, but might give more room for improvement for the children in the control schools compared with the full and partial HPSF, which might have resulted in an underestimation of the effects. To deal with the limitation of not randomizing, all analyses were controlled for gender, study year at baseline, BMI z-score at baseline, SES, and ethnicity. In addition to this lack of randomization, another aspect of the study design should be discussed: the inclusion of only four intervention and four control schools. Due to limitations in resources (money, time, manpower), it was perceived as undesirable to include more schools in the implementation of HPSF. This had the advantage of creating the possibility to get to know the people in the schools and to obtain a detailed understanding of each school context. It also created the time to assess the effects of HPSF in all children in these schools without overburdening the researchers. It enabled us to put more effort into the recruitment of the children and their parents for participation in the measurements in each school. This resulted in a high number of children (60.3\%) who enrolled in the study, and a low drop-out rate over the years.

\section{Data collection}

Applying CARA required a thorough insight into the school context. Fully assessing and understanding all aspects of each school context was deemed impossible. Therefore, we followed research suggestions and focused on the contextual aspects that are indicated as relevant for school health promotion. Even though more insight into, e.g., leadership of the school coordinators, could have contributed to an even better understanding of the school context, the contextual aspects that were assessed provided a deep insight into the four school contexts. We combined appropriate quantitative and qualitative methods, such as questionnaires, interviews, observations, and minutes of meetings. Due to these mixed methods, we were able to employ the principle of data triangulation, which is a strategy that facilitates the validation of data through crossverification from different sources [103].

In addition, a combination of data sources was not only used to assess the context, but also to obtain information about the children's health behaviours. Accelerometers were used, which objectively measured children's PA behaviours, along with parent and child questionnaires. These different sources helped to gain a better insight into the actual dietary and PA behaviours of children throughout the day. To evaluate the effects of HPSF, many different outcomes were assessed rather than just one. To deal with possibly overburdening the participating children, parents, and teachers, we scheduled all annual effect measurements in a school in a single week. Since we used the same weeks each year to conduct measurement, seasonal effects were reduced. The 
disadvantage of this measurement schedule was that the researchers were present in the school for the whole week. This could have influenced the children's health behaviours during that measurement week and might have biased the effects, for example on their PA behaviours. However, the researchers' influence was reduced by the quasi-experimental study design since their presence in school was comparable in the intervention and control schools.

\section{Data analyzing}

Since each school can be considered a complex adaptive system, the control schools should also be considered as such. However, since we focused on a thorough understanding of the contexts and changes in the four intervention schools, we had only a limited focus on the specific school context in the control schools. To evaluate the effects of HPSF, the control schools were combined into one 'control group' in the analyses. This means that their unique contexts were not taken into account, which can be seen as a limitation. Moreover, linear mixed model analyses were conducted to study the longitudinal intervention effects of HPSF. This analysis technique deals with the dependency in the data that is created by the repeated measurements of participants. But it suggests a linear cause-effect relationship, which is in contrast to the nonlinearity of intervention effects in a school system. Thus, it is actually suboptimal for evaluating complex adaptive systems, but a better option was not yet embedded in our way of working. Recently, system dynamics modelling has been increasingly suggested as a promising innovative method [89]. This method has already been applied successfully to other sectors such as engineering, economics, defence, ecology, and business, and is underpinned by a mathematical theory of nonlinear dynamics [168]. System dynamics modelling makes use of causal loop diagrams, which aim to represent the feedback structure of a system by identifying the key variables and indicating the causal relationships between them. Systems modelling in public health research is still in its early stages and includes several limitations linked to, amongst others, model validity [169]. Future studies should deal with these limitations and investigate whether this method can properly analyze HP initiatives in schools.

Involvement of researchers in HPSF

Applying CARA implies that we, as researchers of HPSF, were actively participating partners in the initiative. We joined in the discussions and gave support to the schools whenever possible on the basis of our professional knowledge, skills, and experiences, as well as the results of the monitoring data. This is comparable to the role of researchers in developmental evaluation [48, 170]. As a consequence of this more involved role, the research on HPSF was more time-consuming, as, e.g., a thorough insight into the school context was necessary, which required a relationship of trust with the schools and all other partners involved. This took time to build. Moreover, a more flexible time planning was needed. We had to react quickly to what happened in 
each school. To be able to give relevant support, analysis of the data had to be done quickly to translate it into real-time feedback for the schools. At the same time, the feedback process needed to take place in a careful manner, as both the initiative and the research benefitted from an open discussion of the real situation of those involved without losing the trust of the informants. Finally, even though we were more involved in the process of change, we had to remain objective when conducting the evaluations. By conducting the process evaluation first and then the effect evaluation with a quasiexperimental study design, we attempted to find this balance.

\section{Guiding principles for a CARA researcher}

The abovementioned experiences may help and inspire other health-promotion researchers to evaluate change in a complex adaptive system. On top of these described experiences, I have formulated several guiding principles, which may aid other health-promotion researchers who want to adopt CARA as their research approach.

1. Provide support to the innovation: As a CARA researcher, you should join in the discussions and support the innovators whenever possible to further improve the innovation. In other words, the development of the innovation should become a co-creation, in which evaluation is also part of the process of change.

2. Be receptive, patient, and flexible: As a CARA researcher, you should be receptive to all kinds of interactions in or with the system. You also need to be patient and flexible enough to pay attention to whatever happens and expect the unexpected. Since a process of change in a complex adaptive system requires time, this should be accounted for in the planning of your study.

3. Embrace complexity: As a CARA researcher, you should embrace the complexity of a system instead of thinking in the more traditional linear causal models. This means a shift from a focus on 'one size fits all' evidence-based interventions, to a more flexible perspective of adapting interventions to the different contexts.

4. Do not keep the context in the background: As a CARA researcher, you should not keep the context in the background of an intervention anymore, but it should become the 'foreground'. The research should thus not merely focus on intervention evaluation, but also on context evaluation. This includes a thorough understanding of the pre-existing context and a continuous monitoring of the context during the process of change. It also includes examining the effects separately for each context. This context-oriented evaluation can also provide insight into which contextual aspects to focus on or intervene in to optimize the effects of an intervention. 
5. Use a research diary: As a CARA researcher, you should capture the events in the system, since even a small event can be important to the process of change as it can create that one tipping point that shifts the system. To be able to study retrospectively which events or changes were important, it is recommended to document all observations in a research diary.

6. Accept the consequences of bottom-up involvement: As a CARA researcher, you should accept not having full control over what happens due to bottom-up involvement. It is possible that due to trial and error and feedback loops, (major) adjustments may have to be made to the innovation to create a better fit to the local context. You need to accept this and be prepared to deal with these adjustments.

7. Focus on the right evaluation questions: As a CARA researcher, you should embrace the complexity of the system, in which the intervention is implemented. The aim should be to identify if and how the intervention contributes to reshaping the system in favourable ways, instead of asking whether the intervention works to fix a problem.

Funding bodies can also stimulate health-promotion researchers to use CARA when evaluating change in a complex adaptive system. They can, for example, require that researchers focus on input, output and several types of outcomes, incorporate the local context in their studies, always include a process evaluation, and provide the researchers with more time to investigate contextual aspects and the process of change in a system.

\section{General conclusion}

The aim of this dissertation was to evaluate the Healthy Primary School of the Future in four complex adaptive school systems. The decision to adopt the theoretical perspective of considering schools as complex adaptive systems, means that schools are viewed as systems that consist of many interacting components and have the capability to self-organize and adapt. Embracing this perspective led to the development of HPSF and the programme theory. The latter acted as a conceptual basis for the overall research approach.

The research on HPSF showed that taking the context into account is key to integrating health promotion in schools, as it interacts with the HP changes and influences the development, implementation and effects of HPSF. Moreover, the provision of a daily healthy lunch at school can create a positive disruption (at least in the Netherlands), which can act as a catalyst for wider health promotion in the school system. A shared vision among all people involved and collaboration with external partners were important for the integration of HPSF. In addition, a co-creation process 
was crucial to implementing the HP changes. This process included bottom-up involvement, top-down advice and external practical support and consisted of trial and error, feedback loops, and communication between all people involved. This process helped the schools to adapt the HP changes in a way that fits best to their context. HPSF has led to several effects on children's health and health behaviours. It improved their dietary and PA behaviours, with the largest improvements being seen with the full HPSF. These effects on children's health behaviours resulted in a healthier weight status, which indicates a change from the increasing BMI trend observed in the control schools. To enable children to make healthier choices at home as well, more consistency should be created between these two main microsystems of a child. Moreover, the moderating role of the school context on the effects of HPSF underlines that a broad insight into the school context is crucial to both the implementation of HP changes and a proper understanding of the effects.

Applying a contextual action-oriented research approach enabled us to investigate how HPSF was implemented in the schools, the extent to which HPSF had positively contributed to improved health and health behaviours of children, and where, for whom and in which context it was most effective. Applying this research approach contributed to a broad insight into each school context and the interaction of HPSF with these contexts and generated experiences with evaluating change in a complex adaptive system. The research approach had consequences for all aspects of the research and the role of the researchers themselves.

Overall, it can be concluded from this dissertation that 1) HPSF can be considered a promising initiative in the Netherlands to improve children's health and health behaviours in different school contexts and that 2) CARA can be a possible solution for evaluating change in such complex adaptive school systems. This dissertation shows that embracing the complex and adaptive nature of schools can contribute to a better integration and evaluation of health promotion in schools. 



\section{Valorization Addendum}

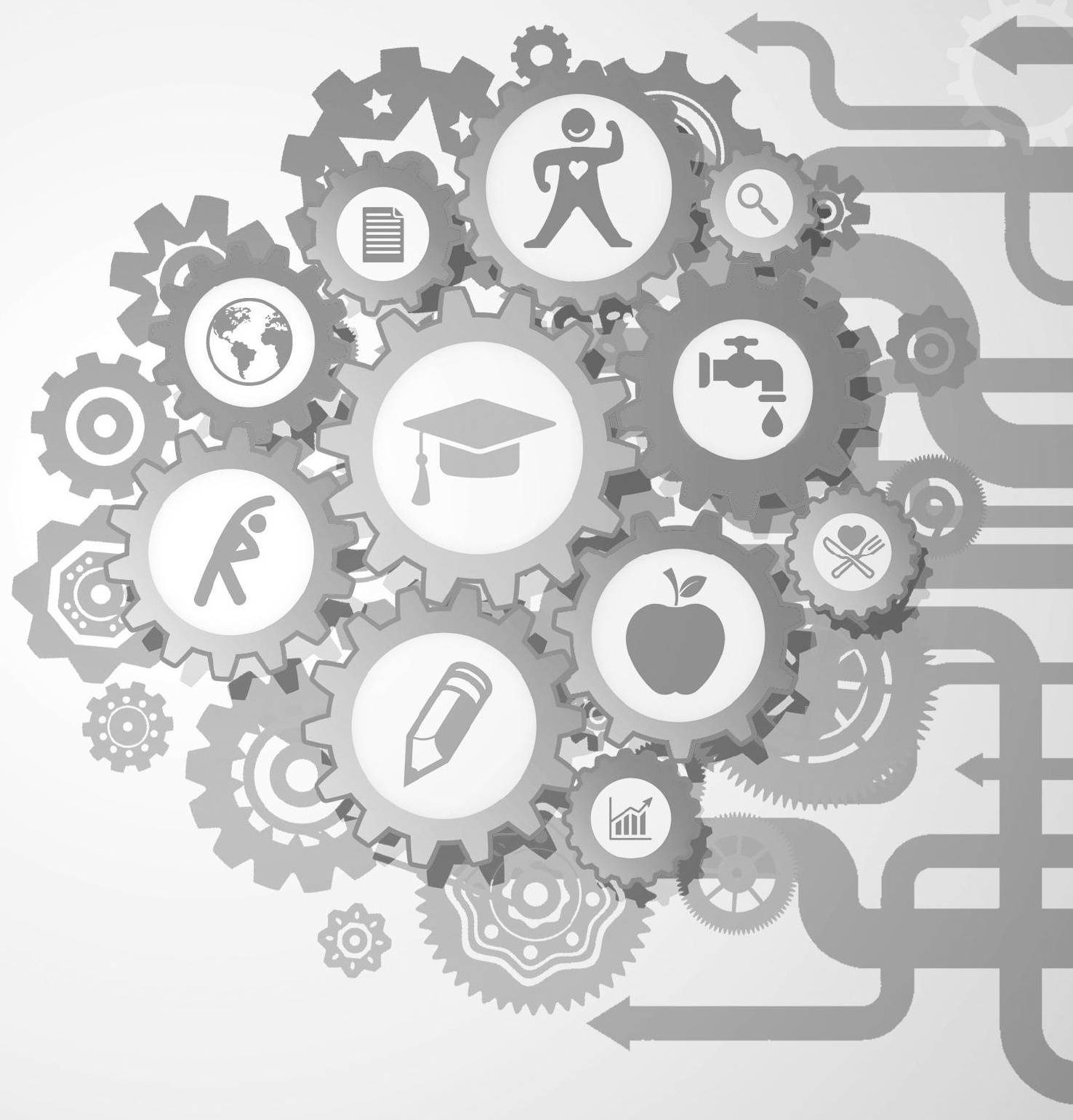



This chapter discusses the valorization of the Healthy Primary School of the Future (HPSF), in which HPSF is considered with all its different aspects and strategies, such as creating a positive disruption, considering schools as complex adaptive systems, the specific focus on each context, and the combined top-down/bottom-up approach. It elaborates on the practical value of HPSF for the four participating schools, and the value for research and practice at a regional, national, and international level. Additionally, the chapter discusses the valorization of the contextual action-oriented research approach (CARA) that was applied to evaluate HPSF.

\section{Valorization of HPSF}

\section{Continued implementation in the four participating schools}

HPSF has been implemented in four primary schools in the Parkstad region in the southern part of the Netherlands. All four participating schools have committed to continued implementation in 2020, when funding is ended, to make the changes sustainable in their school. Meetings with parents, teachers, and external partners are held in each school to examine the best possible way how to sustain all changes. This focus on each specific school context and the bottom-up involvement to sustain the changes have already been important aspects during the development and implementation of HPSF. In each phase of HPSF, the people in the school are part of the process and each context is treated as a unique case. To fit the research to this, we have applied CARA, which aimed to have a specific focus on contextual differences and to support the schools during their process of change. CARA has contributed to the (continued) implementation of the schools by providing regular feedback about the evaluation results. The feedback helped the schools to optimize the health-promoting (HP) changes and to deal with perceived barriers. In addition, the research results showed the people in the four schools that HPSF had favourable effects in their school on the health and health behaviours of children, which increased their motivation to continue the implementation of HPSF. We used several channels to communicate the study findings to the different people in the four schools. Feedback to the school coordinators and the project team existed of, e.g., written summaries of the most important results of the interviews and overviews of the school-specific perceived barriers of the teachers and external pedagogical employees. We informed regularly all teachers and parents in the participating schools about latest developments and recent study findings by launching a website: www.degezondebasisschoolvandetoekomst.nl. In addition, we developed short, easily understandable animated videos to inform all people in the schools about the most important results of the health and behavioural measures [171], and we developed an infographic to present the main findings after 
two years of follow-up [172]. We also organized an interactive evening to provide an opportunity for all teachers and parents in the four schools to discuss the main findings directly with the researchers.

\section{Great interest in HPSF in the Netherlands}

The main findings in this dissertation have shown that HPSF was able to integrate health promotion in the school systems, which have resulted in favourable effects on children's health and health behaviours. These positive results in the four participating schools have led to several concrete actions and decisions: The Province of Limburg has included the dissemination of HPSF in their agenda of 2019-2023 [173], Movare (the educational board of the four participating schools) intends to implement HPSF in 40 of their primary schools, another educational board in the province of Limburg decided to implement an adapted version of HPSF in some of their primary schools, and the municipality of Venlo (northern part of the province of Limburg) intends to implement HPSF in all of their 20 primary schools. On top of these concrete examples in the province of Limburg, many other people in the Netherlands have shown great interest in HPSF. People from other primary schools in the Netherlands, but also people from public health services, policy makers, other health promotion researchers, and even two ministers of the national government have visited the four Healthy Primary Schools of the Future. The high number of visit requests and the positive reactions after the visits indicate that it is an inspiration for many people. It has led to several meetings with local, regional, and national government to discuss the (national) dissemination of HPSF and potential funding for it.

The positive results of HPSF did not just lead to this great interest in HPSF. The whole project team have put a lot of effort in the valorization of HPSF in the Netherlands. The four schools were willing to receive visitors frequently in their school. All members of the project team presented at different conferences or meetings in the Netherlands about our experiences with HPSF. We organized a two-yearly conference about HPSF for all people who are involved and/or interested in HPSF. We invited journalists to publish about HPSF in local, regional, and national newspapers in the Netherlands, and have invited people from the news, including the news specifically for children, to make an item about HPSF. The broad interest in HPSF has resulted in that after a while we did not have to invite these journalists and news-reporters anymore, but they came to us. Furthermore, the website, the factsheet, and the animated videos were disseminated on social media to inform people in the Netherlands about the latest developments and the study results. A public-friendly book was written and disseminated, which describes from the start the whole process of change and its impact in the four schools [174]. In addition, to specifically reach other health 
promotion researchers, we also presented the study findings on several national scientific conferences and have published in Dutch scientific journals.

\section{HPSF across the border}

The interest for HPSF exists also at an international level. The Schools for Health in Europe Network Foundation (SHE) and the UNESCO Chair 'Global Health and Education', have been following this initiative with great interest. We have put a lot of effort in these international networks as well. Not only by publishing the reported studies in this dissertation in international peer reviewed journals, but also by several work visits abroad, participation in SHE activities, and presentations about HPSF on international scientific conferences.

\section{Valorization of CARA}

CARA has been developed to deal with the complex and adaptive nature of school systems. By applying this research approach, we were able to support the participating schools in their process of change and to conduct a thorough evaluation of the process and its final outcomes which addresses the importance of the implementation context. Since we only recently published about CARA in an international peer reviewed journal, it is too early to say whether other (inter)national health promotion researchers have applied this approach also in their studies. However, different people from both research and practice have shown great interest in it and several researchers have the intention to apply CARA in their study and have asked for advice. The interest was not only restricted to Health Promoting School initiatives, but also researchers on studies related to, e.g., the worksite, were interested, which indicates that CARA is also applicable to other complex adaptive systems. Finally, CARA is also being incorporated in the master Health Education and Promotion at Maastricht University to teach future health promoters about this innovative research approach. 



\section{References}

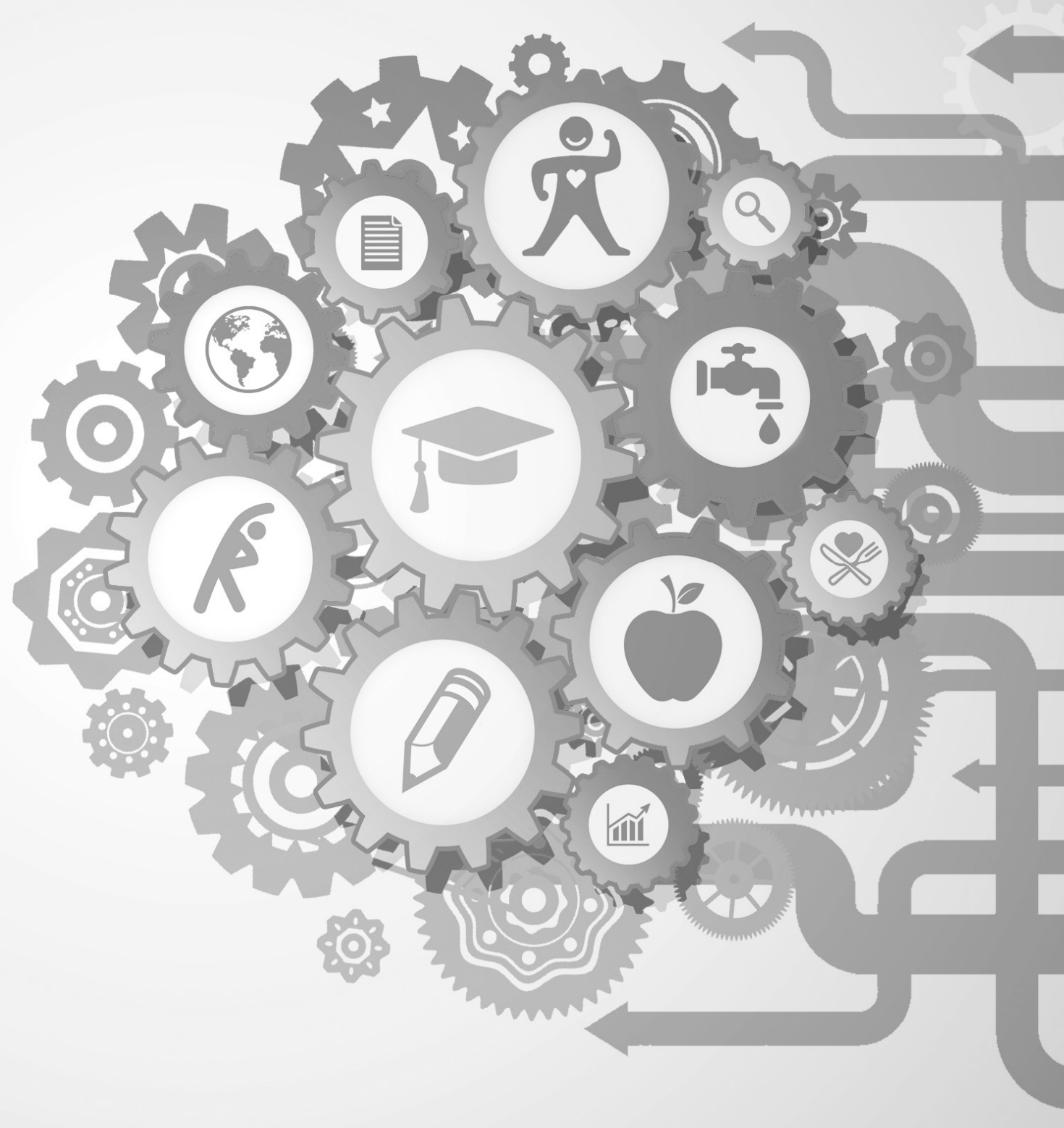


1. Gubbels JS, van Assema P, Kremers SP. Physical activity, sedentary behavior, and dietary patterns among children. Curr Nutr Rep. 2013;2(2):105-12.

2. Sahoo K, Sahoo B, Choudhury AK, Sofi NY, Kumar R, Bhadoria AS. Childhood obesity: causes and consequences. J Family Med Prim Care. 2015;4(2):187.

3. CBS, RIVM, The Netherlands Nutrition Centre. Kinderen eten te weinig fruit, groente en vis [Children eat to less fruit, vegetables and fish] 2017 [Available from: https://www.cbs.nl/nl-nl/nieuws/2017/46/ kinderen-eten-te-weinig-fruit-groente-en-vis.

4. Volksgezondheidenzorg.info. Beweeggedrag kinderen [Physical activity behaviour of children] 2018 [Available from: https://www.volksgezondheidenzorg.info/onderwerp/sport-en-bewegen/cijferscontext/huidige-situatie\#node-beweeggedrag-kinderen.

5. Schönbeck $Y$, Talma $H$, van Dommelen $P$, Bakker B, Buitendijk SE, HiraSing RA, et al. Increase in prevalence of overweight in Dutch children and adolescents: a comparison of nationwide growth studies in 1980, 1997 and 2009. PLoS One. 2011;6(11):e27608.

6. Craigie AM, Lake AA, Kelly SA, Adamson AJ, Mathers JC. Tracking of obesity-related behaviours from childhood to adulthood: a systematic review. Maturitas. 2011;70(3):266-84.

7. Han E, Norton EC, Powell LM. Direct and indirect effects of body weight on adult wages. Econ Hum Biol. 2011;9(4):381-92.

8. Stein D, Weinberger-Litman SL, Latzer Y. Psychosocial perspectives and the issue of prevention in childhood obesity. Front Public Health. 2014;2.

9. Kelsey MM, Zaepfel A, Bjornstad P, Nadeau KJ. Age-related consequences of childhood obesity. J Gerontol. 2014;60(3):222-8.

10. Arcaya MC, Arcaya AL, Subramanian S. Inequalities in health: definitions, concepts, and theories. Glob Health Action. 2015;8(1):27106.

11. Mohammadi NK. Complexity science, schools and health: Applications for management of change in schools to promote health and education. Saarbrucken: Lambert Academic Publishing; 2010.

12. Lynch JW, Kaplan GA, Salonen JT. Why do poor people behave poorly? Variation in adult health behaviours and psychosocial characteristics by stages of the socioeconomic lifecourse. Soc Sci Med. 1997;44(6):809-19.

13. Pampel FC, Krueger PM, Denney JT. Socioeconomic disparities in health behaviors. Annu Rev Sociol. 2010;36:349-70.

14. Dooris M, Poland B, Kolbe L, De Leeuw E, McCall DS, Wharf-Higgins J. Healthy settings. Global perspectives on health promotion effectiveness. Berlin, Germany: Springer; 2007. p. 327-52.

15. Bonell C, Parry W, Wells H, Jamal F, Fletcher A, Harden A, et al. The effects of the school environment on student health: a systematic review of multi-level studies. Health Place. 2013;21:180-91.

16. Gubbels JS, Van Kann DH, de Vries NK, Thijs C, Kremers SP. The next step in health behavior research: the need for ecological moderation analyses-an application to diet and physical activity at childcare. Int J Behav Nutr Phys Act. 2014;11(1):52.

17. Lohrmann DK. A complementary ecological model of the coordinated school health program. Public Health Rep. 2008;123(6):695-703.

18. Hollar D, Lombardo M, Lopez-Mitnik G, Hollar TL, Almon M, Agatston AS, et al. Effective multi-level, multi-sector, school-based obesity prevention programming improves weight, blood pressure, and academic performance, especially among low-income, minority children. J Health Care Poor Underserved. 2010;21(2):93-108.

19. WHO. Promoting health through schools: report of a WHO expert committee on comprehensive school health education and promotion. Geneva; 1997.

20. Deschesnes M, Martin C, Hill AJ. Comprehensive approaches to school health promotion: how to achieve broader implementation? Health Promot Int. 2003;18(4):387-96.

21. WHO. Health promoting schools: A framework for action. Manila, Philippines: World Health Organization Western Pacific Region; 2009. 
22. WHO. Ottawa Charter for Health Promotion: An International Conference on Health Promotion: the Move Towards a New Public Health, November 17-21, 1986, Ottawa, Ontario, Canada: World Health Organization; 1986.

23. WHO. Health Promoting School: an effective approach for early action on NCD risk factors: WHO; 2017 [Available from: https://www.who.int/healthpromotion/publications/health-promotion-school/en/.

24. Turunen H, Sormunen M, Jourdan D, Von Seelen J, Buijs G. Health Promoting Schools-a complex approach and a major means to health improvement. Health promotion international. 2017;32(2):177-84.

25. Leurs MT, Schaalma HP, Jansen MW, Mur-Veeman IM, St. Leger LH, De Vries N. Development of a collaborative model to improve school health promotion in the Netherlands. Health Promot Int. 2005;20(3):296-305.

26. Boot N. Gezondheidsbevordering en voortgezet onderwijs, verstandshuwelijk of echte liefde?: een onderzoek naar de implementatie van schoolgezondheidsbeleid binnen scholen voor voortgezet onderwijs: Maastricht University; 2011.

27. Leurs MTW. A collaborative approach to tailored whole-school health promotion: The SchoolBeat study: Maastricht University; 2008.

28. Pucher KK. Optimizing intersectoral collaboration in school health promotion: creating win-win situations and a systematic implementation based on the diagnosis of sustainable collaboration model: Maastricht University; 2015.

29. Rijksoverheid. Gezonde School programma 2017-2020 [Healthy School Programme 2017-2020]. The Hague, The Netherlands; 2016.

30. Langford R, Bonell CP, Jones HE, Pouliou T, Murphy SM, Waters E, et al. The WHO Health Promoting School framework for improving the health and well-being of students and their academic achievement. Cochrane Database Syst Rev. 2014;4(4):CD008958.

31. Stewart-Brown S. What is the Evidence on School Health Promotion in Improving Health Or preventing Disease And, Specifically, what is the Effectiveness of the Health Promoting Schools Approach? Copenhagen, Denmark: World Health Organization, Regional Office for Europe; 2006.

32. Keshavarz N, Nutbeam D, Rowling L, Khavarpour F. Schools as social complex adaptive systems: a new way to understand the challenges of introducing the health promoting schools concept. Soc Sci Med. 2010;70(10):1467-74.

33. DUO. Aantal scholen in het primair onderwijs 2018 [Available from: https://www.onderwijsincijfers.nl /kengetallen/po/instellingen/aantallen-instellingen-po.

34. Dietz S. Restructuring of Industrial Estates in Parkstad Limburg; Can BIDs contribute to a Relational and Evolutionary Approach? 2010.

35. Elzerman K, Bontje M. Urban shrinkage in parkstad limburg. Eur Plan Stud. 2015;23(1):87-103.

36. Jansen M, Kuppens, E. Op zoek naar de Limburg-factor [seeking the Limburg-factor]. Geleen: GGD Zuid Limburg; 2015.

37. Steenbakkers M VA, Janssen-Goffin MJH, Hajema KJ. Een nieuwe kijk op gezondheid in Zuid-Limburg [A new vision on health in South- Limburg]. In: GGD Zuid-Limburg: Regionaal rapport Volksgezondheid Toekomst Verkenning 2014 p.103. Geleen: GGD Zuid Limburg; 2014.

38. Jansen M, Meisters R. Rapportage nulmeting en monitoring: Sociale Agenda Provincie Limburg. 2018.

39. Jungbluth P. De opbrengst van basisscholen in Zuid-Limburg in 2009 en 2010. [The proceeds of primary schools in South Limburg in 2009 and 2010.]. Maastricht: Maastricht University; 2011.

40. Vermeer AJM, Boot NMWM, Hesdahl MH, Janssen-Goffin MJH, Linssen ECAJ, Rutten N, et al. Lokale rapporten Volksgezondheid Toekomst Verkenning: Een nieuwe kijk op gezondheid in Heerlen, Kerkrade, Landgraaf en Brunssum; [Local reports on Public Health Development: A new perspective on health in Heerlen, Kerkrade, Landgraaf and Brunssum]. Geleen: GGD Zuid Limburg; 2014.

41. Paina L, Peters $\mathrm{DH}$. Understanding pathways for scaling up health services through the lens of complex adaptive systems. Health Policy Plan. 2011;27(5):365-73.

42. Darlington EJ, Violon N, Jourdan D. Implementation of health promotion programmes in schools: an approach to understand the influence of contextual factors on the process? BMC Public Health. 2018;18(1):163. 
43. Moore GF, Evans RE, Hawkins J, Littlecott H, Melendez-Torres G, Bonell C, et al. From complex social interventions to interventions in complex social systems: Future directions and unresolved questions for intervention development and evaluation. Evaluation. 2019;25(1):23-45.

44. Hovmand PS. Introduction to Community-Based System Dynamics. Community Based System Dynamics: Springer; 2014. p. 1-15.

45. Turunen H, Sormunen M, Jourdan D, Von Seelen J, Buijs G. Health Promoting Schools-a complex approach and a major means to health improvement. Health Promot Int. 2017;32(2):177-84.

46. Glouberman S, Zimmerman B. Complicated and complex systems: What would successful reform of Medicare look like? (Discussion Paper No. 8). Ottawa, Ontario: Commission on the Future of Health Care in Canada. 2002

47. Moore GF, Audrey S, Barker M, Bond L, Bonell C, Hardeman W, et al. Process evaluation of complex interventions: Medical Research Council guidance. BMJ. 2015;350:h1258.

48. Patton MQ. Developmental evaluation: Applying complexity concepts to enhance innovation and use. New York, NY, USA: Guilford Press; 2011.

49. Rutter H, Savona N, Glonti K, Bibby J, Cummins S, Finegood DT, et al. The need for a complex systems model of evidence for public health. Lancet. 2017.

50. Egan M ME, Penney T, Anderson de Cuevas R, Er V, Orton L, Lock K, Popay J, Savona N, Cummins S, Rutter H, Whitehead M, De Vocht F, White M, Smith R, Andreeva M, Meier P, Marks D, Petticrew M. NIHR SPHR Guidance on Systems Approaches to Local Public Health Evaluation. Part 1: Introducing systems thinking. London: National Institute for Health Research School for Public Health Research; 2019.

51. Darlington E. Understanding implementation of health promotion programmes: Conceptualization of the process, analysis of the role of determining factors involved in programme impact in school settings: Clermont-Ferrand 2; 2016.

52. Hawe P. Lessons from complex interventions to improve health. Annu Rev Public Health. 2015;36:307-23.

53. Darlington EJ, Simar C, Jourdan D. Implementation of a health promotion programme: A ten-year retrospective study. Health Educ J. 2017;117(3):252-79.

54. Hawe P, Shiell A, Riley T. Theorising interventions as events in systems. Am J Commun Psychol. 2009;43(3-4):267-76.

55. Willeboordse $M$, Jansen $M$, van den Heijkant S, Simons A, Winkens B, de Groot R, et al. The Healthy Primary School of the Future: study protocol of a quasi-experimental study. BMC Public Health. 2016;16(1):1.

56. Marmot M, Allen J, Bell R, Bloomer E, Goldblatt P. WHO European review of social determinants of health and the health divide. Lancet. 2012;380(9846):1011-29.

57. Ogden CL, Carroll MD, Flegal KM. Epidemiologic trends in overweight and obesity. Endocrinol Metab Clin North Am. 2003;32(4):741-60.

58. Suhrcke M, de Paz Nieves C. The impact of health and health behaviours on educational outcomes in high-income countries: a review of the evidence. Copenhagen, Denmark: World Health Organization, Regional Office for Europe 2011.

59. Verrotti A, Penta L, Zenzeri L, Agostinelli S, De Feo P. Childhood obesity: prevention and strategies of intervention. A systematic review of school-based interventions in primary schools. J Endocrinol Invest. 2014;37(12):1155-64.

60. Brown T, Summerbell C. Systematic review of school-based interventions that focus on changing dietary intake and physical activity levels to prevent childhood obesity: an update to the obesity guidance produced by the National Institute for Health and Clinical Excellence. Obes Rev. 2009;10(1):110-41.

61. Pawson R, Tilley N. Realistic evaluation. London, UK: Sage; 1997.

62. Schils T. Leerlingen in het voortgezet onderwijs: wie zitten daar? Hoe zitten ze in hun vel? Hoe slim zijn ze? [Pupils in secondary education: who are they? What is their sense of self-worth? How smart are they?] Kaans reports K04201102-K04201104. Maastricht: Kaans/Maastricht University School of Business and Economics; 2011.

63. Lee A. Health-promoting schools. Appl Health Econ Health Policy. 2009;7(1):11-7. 
64. Boot NM, van Assema P, Hesdahl B, Leurs M, de Vries NK. Gezondheidsbevordering en voortgezet onderwijs: verstandshuwelijk of echte liefde? [Health promotion and secondary education: Marriage of convenience or true love?]. Tijdschr Gezondheidswetenschappen. 2010;88(3):127-35.

65. Mertler CA. Action research: Improving schools and empowering educators. Thousand Oaks, CA, USA: Sage Publications; 2016.

66. Boot N, van Assema P, Hesdahl B, de Vries N. Professional assistance in implementing school health policies. Health Educ J. 2010;110(4):294-308.

67. Hawe P, Shiell A, Riley T. Complex interventions: how" out of control" can a randomised controlled trial be? BMJ. 2004;328(7455):1561.

68. Poland B, Krupa G, McCall D. Settings for health promotion: an analytic framework to guide intervention design and implementation. Health Promot Pract. 2009;10(4):505-16.

69. Michie S, van Stralen MM, West R. The behaviour change wheel: a new method for characterising and designing behaviour change interventions. Implement Sci. 2011;6(1):42.

70. Van Kann DH, Jansen M, De Vries S, De Vries N, Kremers S. Active Living: development and quasiexperimental evaluation of a school-centered physical activity intervention for primary school children. BMC Public Health. 2015;15(1):1315.

71. Fleuren MA, Paulussen TG, Van Dommelen P, Van Buuren S. Towards a measurement instrument for determinants of innovations. Int J Qual Health Care. 2014;26(5):501-10.

72. Nielsen G, Mygind E, Bølling M, Otte CR, Schneller MB, Schipperijn J, et al. A quasi-experimental crossdisciplinary evaluation of the impacts of education outside the classroom on pupils' physical activity, well-being and learning: the TEACHOUT study protocol. BMC Public Health. 2016;16(1):1117.

73. Reiser BJ, Spillane JP, Steinmuler F, Sorsa D, Carney K, Kyza E, editors. Investigating the mutual adaptation process in teachers' design of technology-infused curricula. Fourth international conference of the learning sciences; 2000.

74. Laverack G, Labonte R. A planning framework for community empowerment goals within health promotion. Health Policy Plan. 2000;15(3):255-62.

75. Waterman H, Tillen D, Dickson R, De Koning K. Action research: a systematic review and guidance for assessment. Health Technol Assess. 2000;5(23):iii-157.

76. Green L, Kreuter M. The precede-proceed model. Health promotion planning: an educational approach 3rd ed. Mountain View, CA, USA: Mayfield Publishing Company; 1999. p. 32-43.

77. Konijnendijk AA, Boere-Boonekamp MM, Fleuren MA, Haasnoot ME, Need A. What factors increase Dutch child health care professionals' adherence to a national guideline on preventing child abuse and neglect? Child Abuse Negl. 2016;53:118-27.

78. Verberne LM, Kars MC, Schepers SA, Schouten-van Meeteren AY, Grootenhuis MA, van Delden JJ. Barriers and facilitators to the implementation of a paediatric palliative care team. BMC Palliat Care. 2018;17(1):23.

79. Gevers DW. Parental influences on child snacking: advancing research: Maastricht University; 2016.

80. O'Connor TM, Cerin E, Hughes SO, Robles J, Thompson DI, Mendoza JA, et al. Psychometrics of the preschooler physical activity parenting practices instrument among a Latino sample. Int J Behav Nutr Phys Act. 2014;11(1):3.

81. Gevers DW, Kremers SP, de Vries NK, van Assema P. Patterns of food parenting practices and children's intake of energy-dense snack foods. Nutrients. 2015;7(6):4093-106.

82. Gubbels JS, Sleddens EF, Raaijmakers LC, Gies JM, Kremers SP. The Child-care Food and Activity Practices Questionnaire (CFAPQ): development and first validation steps. Public Health Nutr. 2016;19(11):1964-75.

83. Kremers SP, de Bruijn G-J, Droomers M, van Lenthe F, Brug J. Moderators of environmental intervention effects on diet and activity in youth. Am J Prev Med. 2007;32(2):163-72.

84. Green J, Tones K. For debate. Towards a secure evidence base for health promotion. J Public Health. 1999;21(2):133-9.

85. Slingerland M. Bij modern bewegingsonderwijs wordt.. bewogen!: een praktijkartikel over het intensiveren van de les LO [In modern physical education lessons pupils..move!: a practice-based article about intensivating the physical education lessons]. Lichamelijke opvoeding. 2011;5:28-30. 
86. Langford R, Campbell R, Magnus D, Bonell CP, Murphy SM, Waters E, et al. The WHO Health Promoting School framework for improving the health and well-being of students and staff. Cochrane Database Syst Rev. 2011;1.

87. Fiscella K, Kitzman H. Disparities in academic achievement and health: the intersection of child education and health policy. J Pediatr. 2009;123(3):1073-80.

88. Schaap R, Bessems K, Otten R, Kremers S, van Nassau F. Measuring implementation fidelity of schoolbased obesity prevention programmes: a systematic review. Int J Behav Nutr Phys Act. 2018;15(1):75.

89. Owen B, Brown AD, Kuhlberg J, Millar L, Nichols M, Economos C, et al. Understanding a successful obesity prevention initiative in children under 5 from a systems perspective. PLoS One. 2018;13(3):e0195141.

90. Mason M. What is complexity theory and what are its implications for educational change? Educ Philos Theory. 2008;40(1):35-49.

91. Young I, St Leger L, Buijs G. School health promotion: evidence for effective action. Background paper SHE factsheet. 2013;2.

92. Bartelink N, van Assema P, Jansen M, Savelberg H, Willeboordse M, Kremers S. The Healthy Primary School of the Future: A Contextual Action-Oriented Research Approach. Int J Environ Res Public Health. 2018;15(10):2243.

93. Glasgow RE, Vogt TM, Boles SM. Evaluating the public health impact of health promotion interventions: the RE-AIM framework. Am J Public Health. 1999;89(9):1322-7.

94. Damschroder $\mathrm{L}$, Aron DC, Keith RE, Kirsh SR, Alexander JA, Lowery JC. Fostering implementation of health services research findings into practice: a consolidated framework for advancing implementation science. Implement Sci. 2009;4(1):1.

95. Durlak JA, DuPre EP. Implementation matters: A review of research on the influence of implementation on program outcomes and the factors affecting implementation. Am J Community Psychol. 2008;41(34):327-50.

96. Pearson M, Chilton R, Wyatt K, Abraham C, Ford T, Woods HB, et al. Implementing health promotion programmes in schools: a realist systematic review of research and experience in the United Kingdom. Implement Sci. 2015;10(1):149.

97. Bekker M, Mutsaers B, Dumont R, Boers E, Jansen M. Responsieve evaluatie van Integrale Actie (RIA): een methode voor monitoring door stakeholderdialogen over gezondheidsgerelateerde integrale actie. Tijdschr Gezondheidswetenschappen. 2015;93(6):225-33.

98. Gevers D, Kremers S, de Vries N, van Assema P. The Comprehensive Snack Parenting Questionnaire (CSPQ): Development and Test-Retest Reliability. Int J Env Res Pub He. 2018;15(5).

99. Braun V, Clarke V. Using thematic analysis in psychology. Qual Res Psychol. 2006;3(2):77-101.

100. Lipsey MW. Design sensitivity: Statistical power for experimental research. Pugey, France: Sage; 1990.

101. Shinde S, Weiss HA, Varghese B, Khandeparkar P, Pereira B, Sharma A, et al. Promoting school climate and health outcomes with the SEHER multi-component secondary school intervention in Bihar, India: a cluster-randomised controlled trial. Lancet. 2018;392(10163):2465-77.

102. Gubbels JS, Gerards SM, Kremers SP. Use of food practices by childcare staff and the association with dietary intake of children at childcare. Nutrients. 2015;7(4):2161-75.

103. Boonen A, de Vries N, de Ruiter S, Bowker S, Buijs G. HEPS Guidelines. Guidelines on Promoting Healthy Eating and Physical Activity in Schools. Woerden, The Netherlands: NIGZ; 2009.

104. Rogers EM. Diffusion of preventive innovations. Addict Behav. 2002;27(6):989-93.

105. Strauss RS. Childhood obesity and self-esteem. J Pediatr. 2000;105(1):e15-e.

106. WHO. Report of the commission on ending childhood obesity: World Health Organization; 2016.

107. WHO. Obesity and overweight 2018 [Available from: http://www.who.int/en/news-room/factsheets/detail/obesity-and-overweight.

108. Cook-Cottone C, Casey CM, Feeley TH, Baran J. A meta-analytic review of obesity prevention in the schools: 1997-2008. Psychol Sch. 2009;46(8):695-719. 
109. Oosterhoff M, Joore M, Ferreira I. The effects of school-based lifestyle interventions on body mass index and blood pressure: a multivariate multilevel meta-analysis of randomized controlled trials. Obes Rev. 2016;17(11):1131-53.

110. Bartelink N, van Assema P, Jansen M, Savelberg H, Moore G, Hawkings J, et al. Process evaluation of the Healthy primary School of the Future: The key learning points. BMC Public Health. 2019;19(1):698.

111. Bartelink NH, van Assema P, Kremers SP, Savelberg HH, Oosterhoff M, Willeboordse M, et al. One-and Two-Year Effects of the Healthy Primary School of the Future on Children's Dietary and Physical Activity Behaviours: A Quasi-Experimental Study. Nutrients. 2019;11(3):689.

112. Gezondheidsraad. Dutch Dietary Guidelines 2015. The Hague, The Netherlands: Health Council of the Netherlands; 2015.

113. Shavers VL. Measurement of socioeconomic status in health disparities research. J Natl Med Assoc. 2007;99(9):1013.

114. Keij I. Hoe doet het CBS dat nou? Standaarddefinitie allochtonen [How does Statistics Netherlands do this? Standard definition of emigrants]. 2000.

115. Cole TJ, Bellizzi MC, Flegal KM, Dietz WH. Establishing a standard definition for child overweight and obesity worldwide: international survey. BMJ. 2000;320(7244):1240.

116. Volksgezondheidenzorg.info. Overgewicht kinderen [Overweight children] 2017 [Available from: https://www.volksgezondheidenzorg.info/onderwerp/overgewicht/cijfers-context/huidigesituatie\#node-overgewicht-kinderen.

117. Lister-Sharp D, Chapman S, Stewart-Brown S, Sowden A. Health promoting schools and health promotion in schools: two systematic reviews. In Database of Abstracts of Reviews of Effects (DARE): Qualityassessed Reviews [Internet]. London, UK: Centre for Reviews and Dissemination; 1999

118. Hill JO, Wyatt HR, Melanson EL. Genetic and environmental contributions to obesity. Med Clin North Am. 2000;84(2):333-46

119. Kremers SP, De Bruijn G-J, Visscher TL, Van Mechelen W, De Vries NK, Brug J. Environmental influences on energy balance-related behaviors: a dual-process view. Int J Behav Nutr Phys Act. 2006;3(1):9.

120. Lorenc T, Petticrew M, Welch V, Tugwell P. What types of interventions generate inequalities? Evidence from systematic reviews. J Epidemiol Community Health. 2013;67(2):190-3.

121. Boudewijns E, Pepels J, van Kann D, Konings K, van Schayck C, Willeboordse M. Non-response and external validity in a school-based quasi-experimental study 'The Healthy Primary School of the Future': A cross-sectional assessment. Prev Med Rep. 2019:100874.

122. Langford R, Bonell C, Jones H, Pouliou T, Murphy S, Waters E, et al. The World Health Organization's Health Promoting Schools framework: a Cochrane systematic review and meta-analysis. BMC Public Health. 2015;15(1):130.

123. Shiell A, Hawe P, Gold L. Complex interventions or complex systems? Implications for health economic evaluation. BMJ. 2008:1281-3.

124. Bartelink NH, van Assema P, Kremers SP, Savelberg HH, Oosterhoff M, Willeboordse M, et al. Can the Healthy Primary School of the Future offer perspective in the on-going obesity epidemic in young children? - a Dutch quasi-experimental study. Submitted.

125. Mata J, Silva M, Vieira P, Coutinho S, Andrade A, Teixeira P. Healthy spill-over: Increased exercise motivation improves eating self-regulation during behavioral obesity treatment in women. J Health Psychol. 2009;28:709-16

126. Schulz DN, Kremers SP, van Osch LA, Schneider F, van Adrichem MJ, de Vries H. Testing a Dutch webbased tailored lifestyle programme among adults: a study protocol. BMC Public Health. 2011;11(1):108.

127. Bentsen P, Bonde AH, Schneller MB, Danielsen D, Bruselius-Jensen M, Aagaard-Hansen J. Danish 'addin'school-based health promotion: integrating health in curriculum time. Health Promot Int. 2018.

128. Pluye P, Potvin L, Denis J-L, Pelletier J. Program sustainability: focus on organizational routines. Health Promot Int. 2004;19(4):489-500.

129. Lokale en Nationale Monitor Jeugdgezondheid [Local and National Youth Health Monitor]. Standaardvraagstelling Bewegen [Standard Questionnaire Physical Activity]. [Available from: https://www.monitorgezondheid.nl/jeugdindicatoren.aspx 
130. Craig CL, Marshall AL, Sjorstrom M, Bauman AE, Booth ML, Ainsworth BE, et al. International physical activity questionnaire: 12-country reliability and validity. Med Sci Sports Exerc. 2003;35(8):1381-95.

131. Lokale en Nationale Monitor Jeugdgezondheid [Local and National Youth Health Monitor]. Standaardvraagstelling Voeding [Standard Questionnaire Nutrition] [Available from: https://www.monitorgezondheid.nl/jeugdindicatoren.aspx

132. Van Assema P, Brug J, Ronda G, Steenhuis I. The relative validity of a short Dutch questionnaire as a means to categorize adults and adolescents to total and saturated fat intake. J Acad Nutr Diet. 2001;14(5):377-90.

133. Brink L, Postma-Smeets A, Stafleu A, Wolvers D. Richtlijnen schijf van vijf [Wheel of Five Guidlines]. The Hague: Netherlands Nutrition Centre; 2016.

134. Choi L, Liu Z, Matthews CE, Buchowski MS. Validation of accelerometer wear and nonwear time classification algorithm. Med Sci Sports Exerc. 2011;43(2):357.

135. Jago R, Sebire SJ, Turner KM, Bentley GF, Goodred JK, Fox KR, et al. Feasibility trial evaluation of a physical activity and screen-viewing course for parents of 6 to 8 year-old children: Teamplay. Int J Behav Nutr Phys Act. 2013;10(1):31.

136. Dössegger A, Ruch N, Jimmy G, Braun-Fahrländer C, Mäder U, Hänggi J, et al. Reactivity to accelerometer measurement of children and adolescents. Med Sci Sports Exerc. 2014;46(6):1140.

137. Migueles JH, Cadenas-Sanchez C, Ekelund U, Nyström CD, Mora-Gonzalez J, Löf M, et al. Accelerometer data collection and processing criteria to assess physical activity and other outcomes: A systematic review and practical considerations. Sports Med. 2017:1-25.

138. Evenson KR, Catellier DJ, Gill K, Ondrak KS, McMurray RG. Calibration of two objective measures of physical activity for children. J Sports Sci. 2008;26(14):1557-65.

139. Metcalf B, Henley W, Wilkin T. Effectiveness of intervention on physical activity of children: systematic review and meta-analysis of controlled trials with objectively measured outcomes (EarlyBird 54). BMJ. 2012;345:e5888.

140. Van Kann D, Kremers S, De Vries N, De Vries S, Jansen M. The effect of a school-centered multicomponent intervention on daily physical activity and sedentary behavior in primary school children: The Active Living study. Prev Med. 2016;89:64-9.

141. Shephard RJ. Limits to the measurement of habitual physical activity by questionnaires. Br J Sports Med. 2003;37(3):197-206.

142. Rowland TW. The biological basis of physical activity. Med Sci Sports Exerc. 1998;30(3):392-9.

143. Nederhof AJ. Methods of coping with social desirability bias: A review. Eur J Soc Psychol. 1985;15(3):263-80.

144. Riley AW. Evidence that school-age children can self-report on their health. Ambul Pediatr. 2004;4(4):371-6.

145. Rabia M, Knäuper B, Miquelon P. The eternal quest for optimal balance between maximizing pleasure and minimizing harm: The compensatory health beliefs model. Br J Health Psychol. 2006;11(1):139-53.

146. Golan M, Crow S. Parents are key players in the prevention and treatment of weight-related problems. Nutr Rev. 2004;62(1):39-50.

147. Brookes ST, Whitely E, Egger M, Smith GD, Mulheran PA, Peters TJ. Subgroup analyses in randomized trials: risks of subgroup-specific analyses;: power and sample size for the interaction test. J Clin Epidemiol. 2004;57(3):229-36.

148. O'Connor JP, Temple VA. Constraints and facilitators for physical activity in family day care. Aust J Early Child. 2005;30(4):1-9.

149. Gubbels JS, Kremers SP, Van Kann DH, Stafleu A, Candel MJ, Dagnelie PC, et al. Interaction between physical environment, social environment, and child characteristics in determining physical activity at child care. Health Psychol. 2011;30(1):84.

150. Gubbels JS, Stessen K, van de Kolk I, de Vries NK, Thijs C, Kremers SP. Energy balance-related parenting and child-care practices: The importance of meso-system consistency. PLoS One. 2018;13(9):e0203689.

151. Verjans-Janssen SR, van de Kolk I, Van Kann DH, Kremers SP, Gerards SM. Effectiveness of school-based physical activity and nutrition interventions with direct parental involvement on children's BMI and energy balance-related behaviors-A systematic review. PLoS One. 2018;13(9):e0204560. 
152. Martens M, Van Assema P, Knibbe R, Engels RC, Brug J. Family environmental factors do not explain differences in the behavioral effect of a healthy diet promotion program in lower vocational schools among 12-to 14-year-old adolescents. Am J Health Promot. 2010;24(3):182-5.

153. Snyder S. The simple, the complicated, and the complex: Educational reform through the lens of complexity theory. 2013.

154. Rosas SR. Systems thinking and complexity: considerations for health promoting schools. Health Promot Int. 2015;32(2):301-11.

155. Bisset S, Daniel M, Potvin L. Exploring the intervention-context interface: a case from a school-based nutrition intervention. Am J Eval. 2009;30(4):554-71.

156. Fleuren M, Paulussen T, Van Dommelen $P$, Van Buuren S. Measurement instrument for determinants of innovations (MIDI). Leiden, the Netherlands: TNO; 2014.

157. Kane H, Lewis MA, Williams PA, Kahwati LC. Using qualitative comparative analysis to understand and quantify translation and implementation. Transl Behav Med. 2014;4(2):201-8.

158. Schneider CQ, Wagemann C. Set-theoretic methods for the social sciences: A guide to qualitative comparative analysis: Cambridge University Press; 2012.

159. Burton C. Heavy tailed distributions of effect sizes in systematic reviews of complex interventions. PLoS One. 2012;7(3):e34222.

160. Vermeiren AP, Willeboordse M, Oosterhoff M, Bartelink N, Muris P, Bosma H. Socioeconomic multidomain health inequalities in Dutch primary school children. Eur J Public Health. 2018;28(4):610-6.

161. Ball K. Traversing myths and mountains: addressing socioeconomic inequities in the promotion of nutrition and physical activity behaviours. Int J Behav Nutr Phys Act. 2015;12(1):142.

162. Kremers SP, Visscher TL, Schuit AJ. Effect in zijn context [Effect in its context]. Tijdschr Gezondheidswetenschappen. 2018;96(3-4):128-31.

163. Blakely CH, Mayer JP, Gottschalk RG, Schmitt N, Davidson WS, Roitman DB, et al. The fidelity-adaptation debate: Implications for the implementation of public sector social programs. Am J Community Psychol. 1987;15(3):253-68.

164. Elliott DS, Mihalic S. Issues in disseminating and replicating effective prevention programs. Prev Sci. 2004;5(1):47-53

165. Hirsch GB, Levine R, Miller RL. Using system dynamics modeling to understand the impact of social change initiatives. Am J Community Psychol. 2007;39(3-4):239-53.

166. Jansen M. Lokale overheid en populatiegericht gezondheidsbeleid: Maastricht University; 2015.

167. Pommier J, Guével M-R, Jourdan D. Evaluation of health promotion in schools: a realistic evaluation approach using mixed methods. BMC Public Health. 2010;10(1):43.

168. Atkinson J-A, Wells R, Page A, Dominello A, Haines M, Wilson A. Applications of system dynamics modelling to support health policy. Public Health Res Pract. 2015;25(3):e2531531.

169. Xue H, Slivka L, Igusa T, Huang T, Wang Y. Applications of systems modelling in obesity research. Obes Rev. 2018;19(9):1293-308.

170. Fagen MC, Redman SD, Stacks J, Barrett V, Thullen B, Altenor S, et al. Developmental evaluation: Building innovations in complex environments. Health Promot Pract. 2011;12(5):645-50.

171. De Gezonde Basisschool van de Toekomst. Onderzoek 16/17 Interventiescholen 2017 [Available from: https://www.youtube.com/watch?v=j-OAEaan0xA.

172. De Gezonde Basisschool van de Toekomst. Infographic 2018 [Available from: http://www. degezondebasisschoolvandetoekomst.nl/upload/documents/\%5BOorspr_\%20grootte\%5D\%20Beweegsc hool\%20factsheet.jpg.

173. Provincie-Limburg. Collegeprogramma 2019-2023: Verniewend Verbinden. Maastricht; 2019.

174. Jansen M, Burhenne K. De Gezonde Basisschool van de Toekomst: van leer- naar leefschool. Maastricht: Provincie Limburg, GGD Zuid Limburg; 2019. 



\section{Summary}

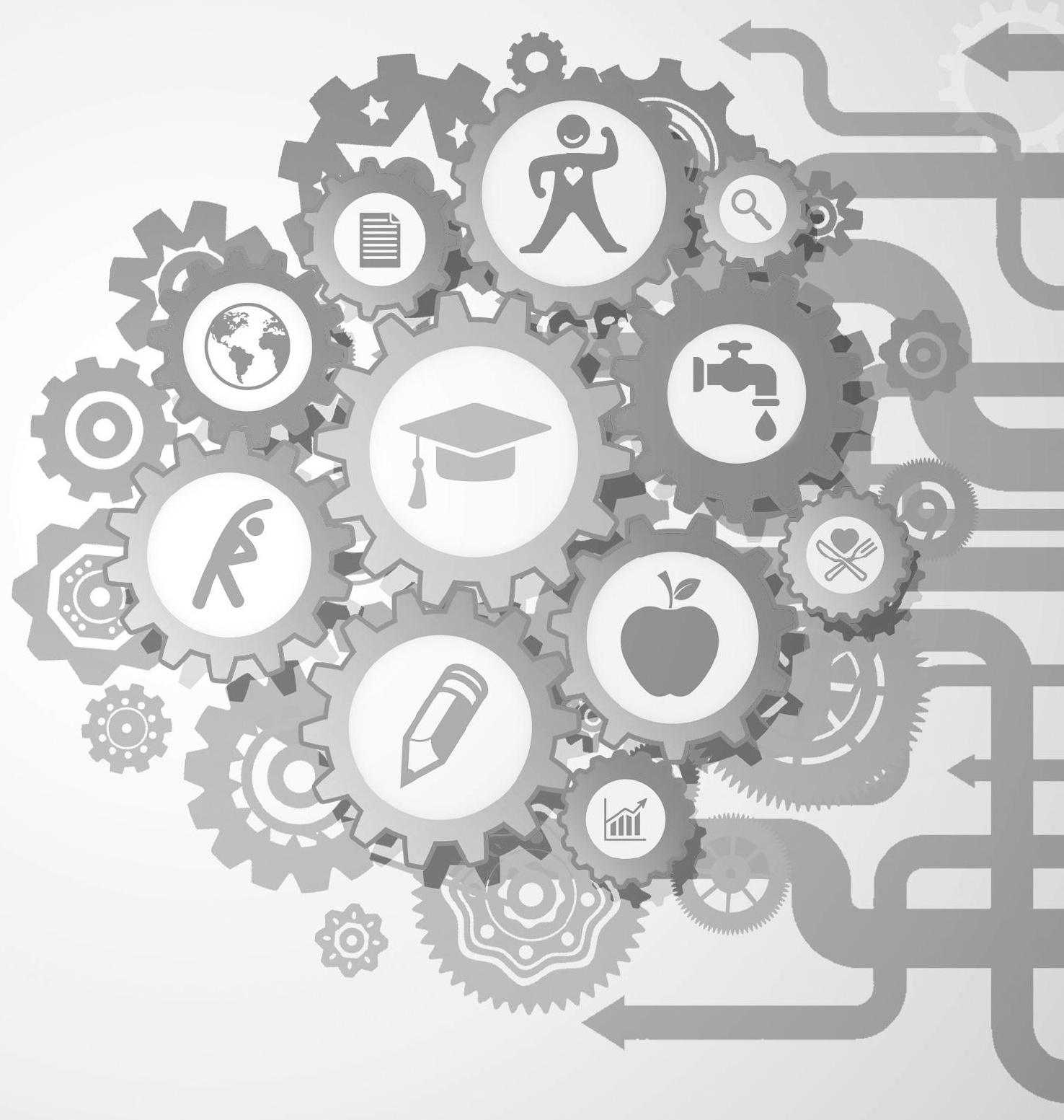



The aim of the research presented in this dissertation was to evaluate the Healthy Primary School of the Future (HPSF) in four complex adaptive school systems. HPSF is a Dutch initiative based on the principles of the Health Promoting School framework that aims to integrate health and well-being within the whole school system. The initiative intends to establish a broad collaboration between school, parents, and external partners, which should lead to a co-creation movement in schools consisting of topdown and bottom-up processes. HPSF intends to create some form of positive disruption in the schools by initiating two health-promoting (HP) changes top-down: 1) a free healthy lunch each day and 2) daily structured physical activity (PA) and cultural sessions after lunch. This should create momentum for more bottom-up processes to implement additional HP changes. All HP changes together should favourably affect the health behaviours of the school children, leading to improved health and wellbeing of these children.

In this evaluation, schools are considered as complex adaptive systems, which means that they consist of many interacting components and have the capability to selforganize and adapt. Embracing this perspective to schools led to the development of HPSF and a programme theory. The programme theory acted as a conceptual basis for the contextual action-oriented research approach (CARA).

Chapter 2 elaborates on CARA in more detail. The purpose of CARA for researchers is to contribute to a health promoting school initiative and to conduct a thorough evaluation at the same time. Basic properties of CARA are its specific focus on contextual differences and the use of monitoring and feedback to both support and evaluate the process of change. The approach centers around four key questions: 1) What is the pre-existing context of each school? 2) How does the process of change in each school evolve and which factors affect this process? 3) How can research contribute to the process of change?, and 4) Do children's health and health behaviours improve as a result of the HP changes?

The studies reported in this dissertation applied CARA to evaluate how HPSF was implemented in the schools, to what extent HPSF had positively contributed to improved health and health behaviours of children, and where, for whom and in which context it was most effective.

Chapter 3 presents the results of the process evaluation of HPSF. The study explored the processes through which HPSF and each school context adapted to one another in the four intervention schools. Results showed that two of the four intervention schools decided to implement the two top-down changes ('full HPSF'), i.e., the lunch and the structured PA and cultural sessions. To realize these changes during the lunch break, both schools extended the lunch break time and their school day. Both schools also implemented several additional HP changes: they provided water bottles to all children, improved their school's health policy, and started with an educational lunch. The other two intervention schools decided to only implement the structured PA and cultural sessions each day ('partial HPSF'). They did not provide a healthy lunch nor 
did they increase their lunch break time or implement additional HP changes. Results showed that the provided lunch created a positive disruption and acted as a catalyst for wider school health promotion efforts. Conditions that enhanced the implementation of the HP changes were sufficient bottom-up involvement, external support, team cohesion and coordination in school for all HP changes. Additionally, the focus on each specific school was crucial for the implementation of HPSF, as each school had their own starting point and process of change.

Chapter 4 describes the effects of HPSF on children's Body Mass Index (BMI) z-score after one and two years' follow-up and shows whether the effects of HPSF were moderated by children's gender, age, socioeconomic status (SES), or weight status. Children's BMI z-scores in both the full HPSF (effect size $(E S)=-0.08$ ) and the partial HPSF (ES=-0.07) significantly decreased after two years' follow-up compared with children of the control schools, whose mean BMI z-score increased from baseline to two years. After one-year follow-up, this favourable effect was already significant in the partial HPSF (ES=-0.05), but not yet in the full HPSF (ES=-0.04). No specific subgroups of children could be identified who benefitted more from HPSF. The findings suggest that both versions of HPSF, and especially the full HPSF, seem promising in offering perspective in the on-going obesity epidemic in young children.

Chapter $\mathbf{5}$ shows the effects of HPSF on children's dietary and PA behaviours after one and two years' follow-up. Significant favourable effects were found in the full HPSF for, among others, school water consumption (effect size $(E S)=1.03(T 1), 1.14$ (T2)), lunch intake of vegetables (odds ratio $(\mathrm{OR})=3.17(\mathrm{~T} 1), 4.39(\mathrm{~T} 2)$ ) and dairy products (OR $=4.43(\mathrm{~T} 1), 4.52(\mathrm{~T} 2))$, sedentary time ( $\mathrm{ES}=-0.23(\mathrm{~T} 2))$, and light PA (ES = $0.22(\mathrm{~T} 2)$ ). Hardly any significant favourable effects were found for the partial HPSF. The findings in this study suggest that focusing on both nutrition and PA components seems to be more effective in promoting children's healthy behaviours than focusing exclusively on PA.

Chapter 6 presents the results of the two-year effects of HPSF on children's dietary and PA behaviours at school and at home, and shows whether child characteristics (gender, age, and weight status) or the home environment (SES, patterns of nutritionrelated and PA-related parenting practices) moderated these effects. Favourable effects on children's dietary and PA behaviours at school were found in the full HPSF; in the partial HPSF on PA behaviours. Children in the full HPSF did not compensate at home for the health-promoting school activities, while in the partial HPSF the children became less active at home. In both the full and partial HPSF, less favourable effects at school were found for younger children. At home, less favourable effects were found for children with a lower SES. Overall, the effect of the full HPSF was larger and more equally beneficial for all children than that of the partial HPSF.

Chapter 7 describes the moderating role of the school context on the effects of HPSF among the four intervention schools. Potentially moderating contextual aspects were found on the level of the children, i.e., children's socioeconomic background and their baseline health behaviours, the employees, i.e., teachers' HP practices and the 
external pedagogical employees' perceived barriers, and the school itself, i.e., whether existing organizational issues were synergized with HPSF. The results showed that similar HP changes led to different outcomes across schools due to differences in the school context. The adoption of a complex adaptive systems perspective contributes to a better understanding of the variation in effects across schools and can provide insight on which contextual aspects to focus on or intervene in to optimize the effects of HP initiatives.

Chapter 8 discusses the most important findings from the reported studies. The main findings and our experiences are used to reflect on the research approach and the theoretical perspective, and to provide an overall conclusion. The reported studies suggest that taking the school context into account is key to integrating HPSF in schools as it continuously interacts with HPSF and influences its development, implementation and effects. Moreover, a HP change that is disruptive to the school system, extended school time, and a co-creation process including bottom-up involvement and external support contributed to the integration of HPSF in the schools. HPSF has led to improved dietary and PA behaviours of children, with largest improvements in the full HPSF. These effects on children's health behaviours have resulted in a healthier weight status, which indicates a change from the increasing BMI trend observed in the control schools. Concerning the research approach, it is suggested that CARA contributed to a broad insight into each school context and the interaction of these contexts with HPSF. The research approach had consequences for all aspects of the research and the role of the researchers themselves. It has generated experiences with evaluating change in a complex adaptive system.

Overall, it is concluded from the studies in this dissertation that 1) HPSF can be considered a promising initiative in the Netherlands to improve children's health and health behaviours in different school contexts and that 2) CARA can be a solution for evaluating change in such complex adaptive school systems. This dissertation shows that embracing the complex and adaptive nature of schools can contribute to a better integration and evaluation of health promotion in schools. 



\section{Samenvatting}

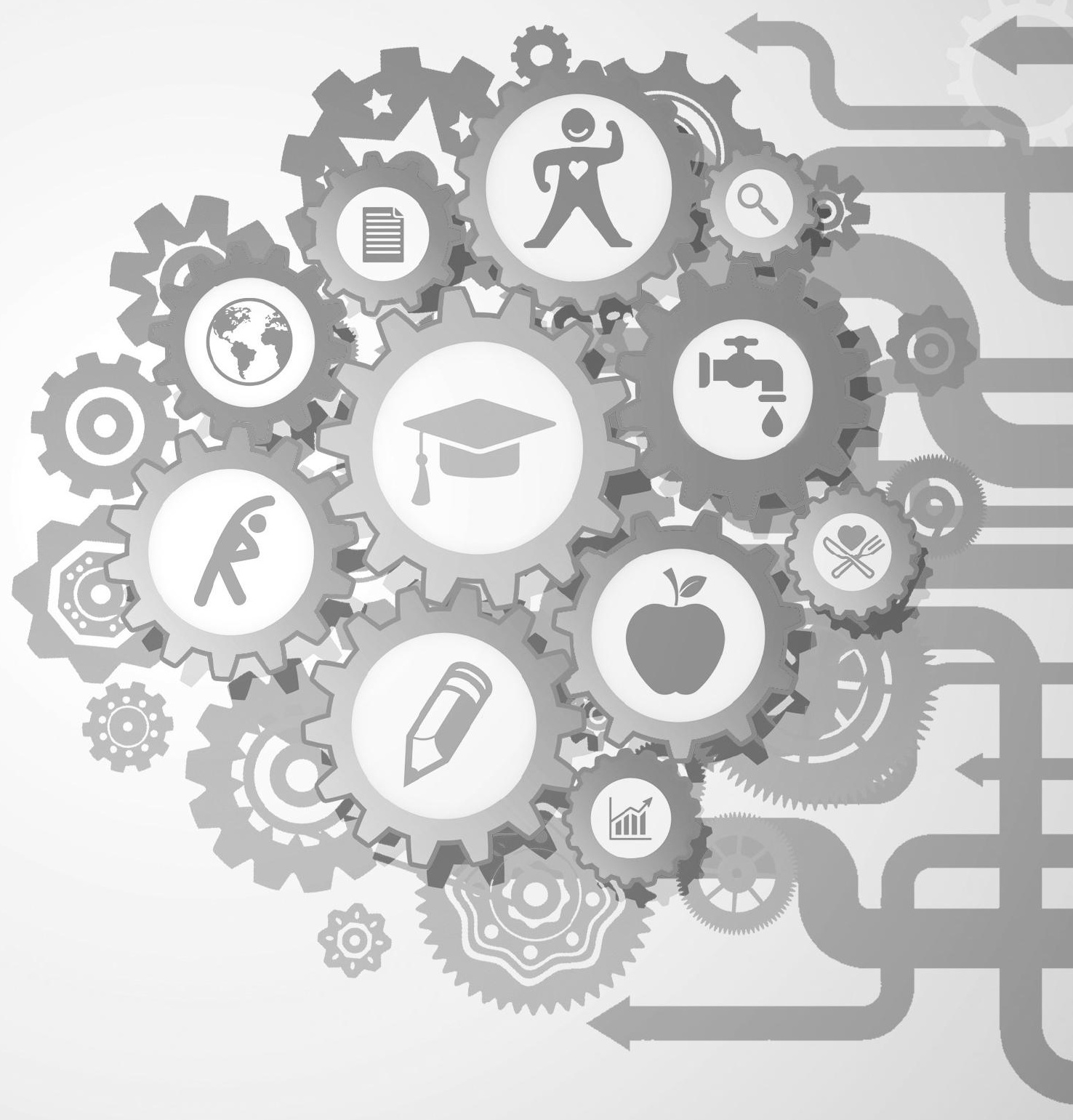


Het doel van dit proefschrift is om de Gezonde Basisschool van de Toekomst (Engelstalige afkorting: HPSF) te evalueren in vier scholen. HPSF is een Nederlands initiatief dat is gebaseerd op de principes van de Gezonde School benadering en heeft als doel gezondheid en welzijn te integreren in het gehele schoolsysteem. Het idee achter HPSF is om een co-creatie tot stand te brengen waarin scholen, ouders en externe partners samenwerken. Daarbij is het idee om een positieve disruptie in de scholen te bewerkstelligen door middel van twee van bovenaf bedachte gezondheidsbevorderende veranderingen: 1) iedere dag een gratis gezonde lunch en 2) dagelijkse gestructureerde beweeg- en culturele activiteiten na de lunch, beide begeleid door pedagogisch medewerkers van kinderopvangorganisaties. Deze twee veranderingen zouden moeten leiden tot een momentum voor het uitvoeren van additionele gezondheidsbevorderende veranderingen in de school. Het idee is dat alle veranderingen samen het gezondheidsgedrag van kinderen verbeteren. Dit zou vervolgens moeten leiden tot meer optimale gezondheid en welzijn van kinderen.

Scholen worden in dit proefschrift beschouwd als complexe adaptieve systemen. Dit betekent dat scholen worden gezien als systemen die zich kunnen aanpassen aan allerlei omstandigheden, die zichzelf kunnen organiseren en die bestaan uit allerlei componenten die met elkaar in interactie zijn. Het omarmen van deze zienswijze heeft geleid tot de ontwikkeling van HPSF en een programmatheorie. Deze programmatheorie heeft gediend als conceptuele basis voor de overkoepelende contextueel actiegerichte onderzoeksbenadering (Engelstalige afkorting: CARA).

Hoofdstuk 2 gaat dieper in op CARA. Het doel van CARA is om als onderzoekers bij te dragen aan een gezonde-schoolinitiatief en tegelijkertijd een gedegen onderzoek uit te voeren naar de processen en effecten van HPSF. Kerneigenschappen van CARA zijn de specifieke focus op de contextuele verschillen tussen scholen en het gebruik van monitoring en feedback om de veranderingsprocessen op de scholen te ondersteunen en te evalueren. In CARA staan vier kernvragen centraal: 1) Hoe ziet de bestaande context van iedere school eruit?; 2) Hoe verloopt het veranderingsproces in iedere school en welke factoren zijn hierop van invloed?; 3) Hoe kan onderzoek bijdragen aan het veranderingsproces in de scholen?; 4) Verbetert de gezondheid en het gezondheidsgedrag van kinderen door de gezondheidsbevorderende veranderingen op school?

De beschreven studies in dit proefschrift hebben deze onderzoeksbenadering toegepast om te evalueren hoe HPSF is uitgevoerd op de scholen, in welke mate HPSF bijdraagt aan het verbeteren van de gezondheid en het gezondheidsgedrag van kinderen, en waar, voor wie en in welke context HPSF het meest effectief is.

Hoofdstuk 3 presenteert de resultaten van de procesevaluatie van HPSF. De studie beschrijft hoe in vier scholen HPSF en de schoolcontext zich hebben aangepast aan elkaar. De resultaten laten zien dat twee van de vier interventiescholen ('volledige HPSF') hebben besloten om de twee van bovenaf bedachte veranderingen door te voeren (de lunch en het gestructureerde beweeg- en cultuuraanbod). Om deze 
veranderingen te realiseren hebben beide scholen hun lunchtijd en schooldag verlengd. Beide scholen hebben ook verschillende additionele gezondheidsbevorderende veranderingen doorgevoerd: ze hebben alle kinderen voorzien van een waterbidon, hebben hun schoolgezondheidsbeleid verbeterd, zoals het traktatiebeleid, en zijn begonnen met een educatieve lunch. De andere twee scholen ('gedeeltelijke HPSF') hebben besloten om alleen het gestructureerde beweeg- en cultuuraanbod door te voeren. Zij hebben geen lunch aangeboden en hebben niets veranderd aan hun schooltijden. Deze twee scholen hebben geen additionele gezondheidsbevorderende veranderingen ingevoerd. De procesevaluatie laat zien dat de lunch een positieve disruptie bewerkstelligde, die werkte als een soort vliegwiel voor verdere gezondheidsbevorderende veranderingen binnen de school. Condities die hebben geholpen om alle gezondheidsbevorderende veranderingen in te voeren waren voldoende betrokkenheid van ouders, leraren en pedagogisch medewerkers in de school, externe ondersteuning, een hecht team en stevige coördinatie bij alle veranderingen in school. Tenslotte bleek aandacht voor de schoolcontext cruciaal, aangezien iedere school zijn eigen startsituatie en veranderingsproces kende.

Hoofdstuk 4 beschrijft het effect van HPSF op de Body Mass Index (BMI) z-score van kinderen na één en twee jaar. De beschreven studie heeft ook onderzocht of de effecten van HPSF werden gemodereerd door geslacht, leeftijd, sociaaleconomische status (SES) of gewichtsstatus. De BMI z-score van kinderen in zowel de volledige interventiescholen (effectgrootte $(E S)=-0.08$ ) als de gedeeltelijke interventiescholen ( $E S=-0.07)$ is significant afgenomen na twee jaar follow-up vergeleken met de kinderen van de controle scholen, waar de BMI z-score zelfs is toegenomen twee jaar na de start. Dit gewenste effect van HPSF was na één jaar follow-up al zichtbaar in de gedeeltelijke HPSF (ES = -0.05), maar nog niet in de volledige HPSF (ES = -0.04). Daarnaast bleken geen specifieke subgroepen van kinderen meer voordeel te hebben van HPSF. De resultaten uit deze studie lijken aan te geven dat beide versies van HPSF veelbelovend zijn in het keren van de trend van de aanhoudend stijgende obesitas epidemie in jonge kinderen. De volledige HPSF lijkt daarin vooralsnog, in de tweejarige follow-up periode, succesvoller dan de gedeeltelijke variant.

Hoofdstuk 5 beschrijft de effecten van HPSF op het eet- en beweeggedrag van kinderen na één en twee jaar follow-up. Significante verbeteringen zijn gevonden voor de volledige HPSF in vergelijking met de controlescholen voor onder andere het drinken van water op school (ES = $1.03(\mathrm{~T} 1), 1.14(\mathrm{~T} 2))$, het tijdens de lunch eten van groente (odds ratio $(O R)=3.17(T 1), 4.39(T 2)$ ) en zuivel $(O R=4.43(T 1), 4.52(T 2)$ ), het zitgedrag $(E S=-0.23(T 2))$ en licht beweeggedrag $(E S=0.22(T 2))$ van kinderen. Zo goed als geen significante verbeteringen zijn gevonden voor de gedeeltelijke HPSF. De studie laat zien dat het richten op zowel voeding als beweging een duidelijke meerwaarde lijkt te hebben dan het alleen richten op het beweeggedrag van kinderen.

Hoofdstuk 6 presenteert de effecten van HPSF na twee jaar follow-up op het eet- en beweeggedrag van kinderen op school en thuis. De beschreven studie heeft ook 
onderzocht of deze effecten worden gemodereerd door karakteristieken van het kind (geslacht, leeftijd, gewichtsstatus) of de thuisomgeving (SES, patronen van voedings- of beweeg-gerelateerde praktijken van ouders). Significante verbeteringen op school zijn gevonden voor de volledige HPSF voor zowel het voedings- als beweeggedrag van kinderen. In de gedeeltelijke HPSF is het beweeggedrag van kinderen op school verbeterd. Kinderen op de volledige HPSF scholen compenseerden hun gedrag thuis niet vanwege de gezondheidsbevorderende activiteiten op school, terwijl de kinderen van de gedeeltelijke HPSF minder actief werden thuis. In beide versies van HPSF zijn de effecten gemodereerd. Op school zijn minder gewenste effecten gevonden voor de jongere kinderen en thuis zijn minder gewenste effecten gevonden voor de kinderen met een lagere sociaaleconomische achtergrond. In het algemeen zijn de effecten op de volledige HPSF groter en meer gelijk voor alle kinderen dan die op de gedeeltelijke HPSF.

Hoofdstuk 7 beschrijft de modererende rol van de schoolcontext op de effecten van HPSF in de vier scholen. Onderdelen van de context die lijken te modereren zijn gevonden op het niveau van het kind (de sociaaleconomische achtergrond van kinderen en hun gezondheidsgedrag bij de start van HPSF), op het niveau van de werknemers (de gezondheidsbevorderende praktijken van leraren en de ervaren barrières van de externe pedagogisch medewerkers) en op het niveau van de school (het creëren van synergie tussen bestaande organisatorische issues en HPSF). De resultaten laten zien dat dezelfde gezondheidsbevorderende veranderingen in scholen leiden tot verschillende effecten in die scholen vanwege de verschillen in hun context. Het omarmen van een complexe, adaptieve systeembenadering voor scholen helpt om de verschillende effecten tussen scholen te begrijpen en kan inzicht geven op welke contextuele aspecten te focussen of te interveniëren om de effecten van gezondheidsbevorderende veranderingen te optimaliseren.

Hoofdstuk 8 bediscussieert de belangrijkste bevindingen van de beschreven studies. Deze bevindingen en de eigen ervaringen zijn gebruikt om te reflecteren op het theoretische perspectief en de onderzoeksbenadering, en om uiteindelijke conclusies te trekken. De beschreven studies laten zien dat voor het integreren van HPSF in scholen het cruciaal is rekening te houden met de schoolcontext. Deze context is continu in interactie met de invoering en ontwikkeling van HPSF en beïnvloedt daardoor de doorontwikkeling, de uitvoering en de effecten ervan. Daarnaast nodigt het creëren van een positieve disruptie in het schoolsysteem uit tot co-creatie tussen de medewerkers op de school en de externe partners om samen een nieuw evenwicht tot stand te brengen in het systeem. Dat co-creëren heeft bijgedragen aan het integreren van HPSF in de scholen. HPSF heeft geleid tot verbeteringen in het eet- en beweeggedrag van kinderen, waarin de grootste verbeteringen te zien waren in de volledige HPSF scholen. Deze effecten op het gezondheidsgedrag van kinderen hebben geresulteerd in een gezondere gewichtsstatus, wat een verandering in de bestaande BMI-trend laat zien die zichtbaar werd in de controlescholen. Kijkend naar de onderzoeksbenadering, blijkt 
CARA bij te dragen aan meer inzicht in de specifieke schoolcontext en de interactie tussen deze schoolcontext en HPSF. De onderzoeksbenadering had consequenties voor alle aspecten van het onderzoek en de rol van de onderzoekers zelf. Het toepassen van CARA heeft geleid tot een breed scala aan ervaringen in het evalueren van veranderingen in een complex adaptief systeem.

Alles in overweging nemend, kan naar aanleiding van de beschreven studies in dit proefschrift geconcludeerd worden dat 1) HPSF een veelbelovend Nederlands initiatief is om de gezondheid en het gezondheidsgedrag van kinderen in verschillende schoolcontexten te verbeteren, en dat 2) CARA een mogelijke oplossing biedt om verandering in zo'n complex adaptief schoolsysteem te evalueren. Dit proefschrift laat zien dat het omarmen van de complexe en adaptieve aard van scholen kan bijdragen aan een betere integratie en evaluatie van gezondheidsbevordering in scholen. 



\section{Curriculum Vitae}

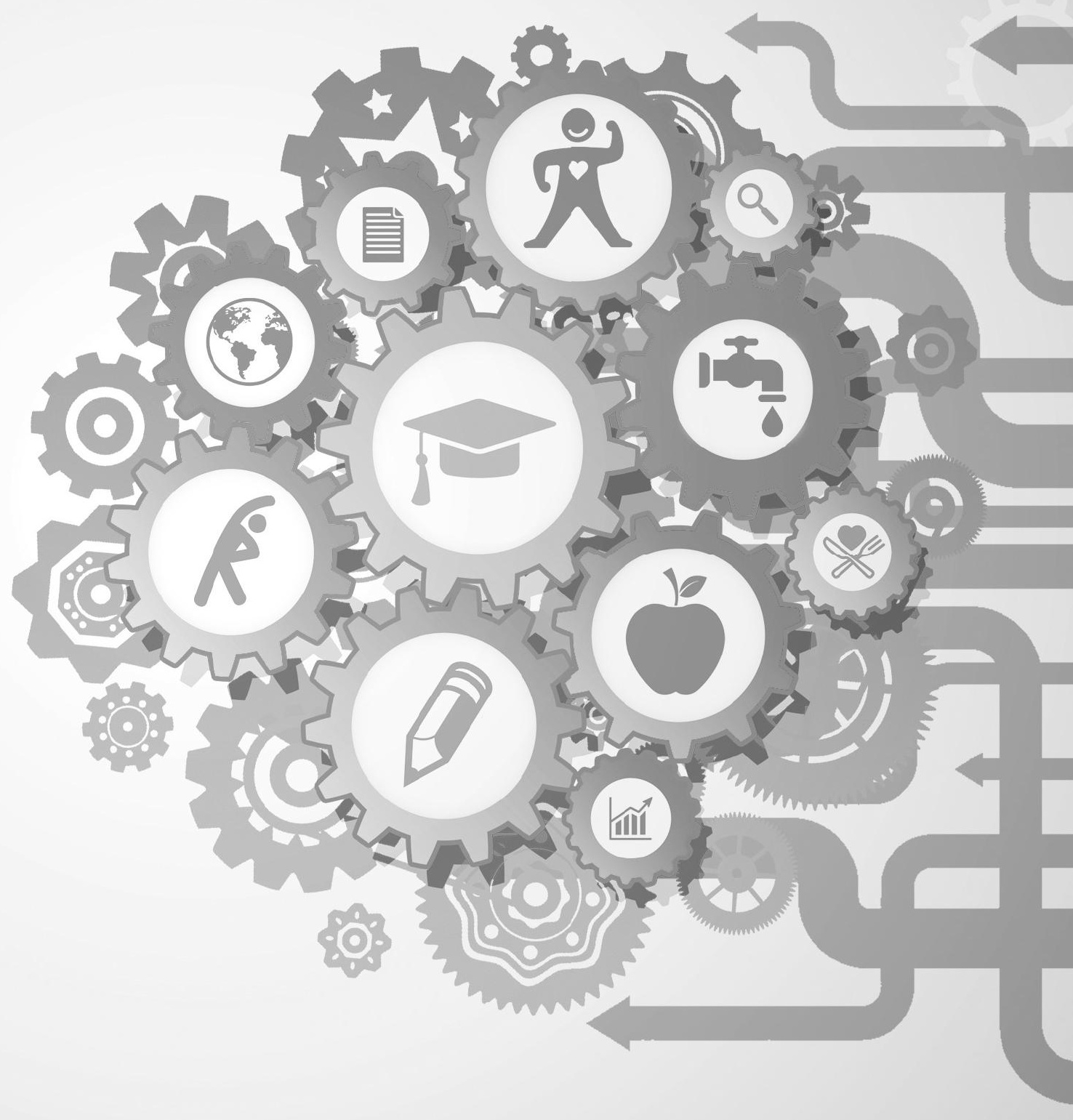



Nina Henrica Maria Bartelink was born on August 181988 in Stein. In 2010, she graduated for the Bachelor Health Sciences at Maastricht University with a major in Movement Sciences and a minor in Bioregulation and Health. In 2011, she graduated from the Master Sports and Physical Activity Interventions at Maastricht University. She started working as junior researcher at the Regional Public Health Services Southern Limburg at the Department of Knowledge and Innovation in January 2012. During this employment, she conducted the evaluation of RealFit and was, among others, involved in the Health Monitors and the regional Public Health Foresight Study (rVTV). In August 2014, she was employed by Maastricht University at the Department of Health Promotion and started as PhD Candidate on the Healthy Primary School of the Future initiative. During her PhD, she presented her work at several (inter)national conferences and had several work visits abroad. She stayed at Cardiff University (Wales) for two months to collaborate with the DECIPHer group (the Centre for the Development and Evaluation of Complex Interventions for Public Health Improvement) at the Department of Social Sciences. In July 2019, she started working as postdoc Health Promoting School at the Department of Health Promotion to collaborate in and give advice to other Health Promoting School initiatives. 



\section{Publication list}

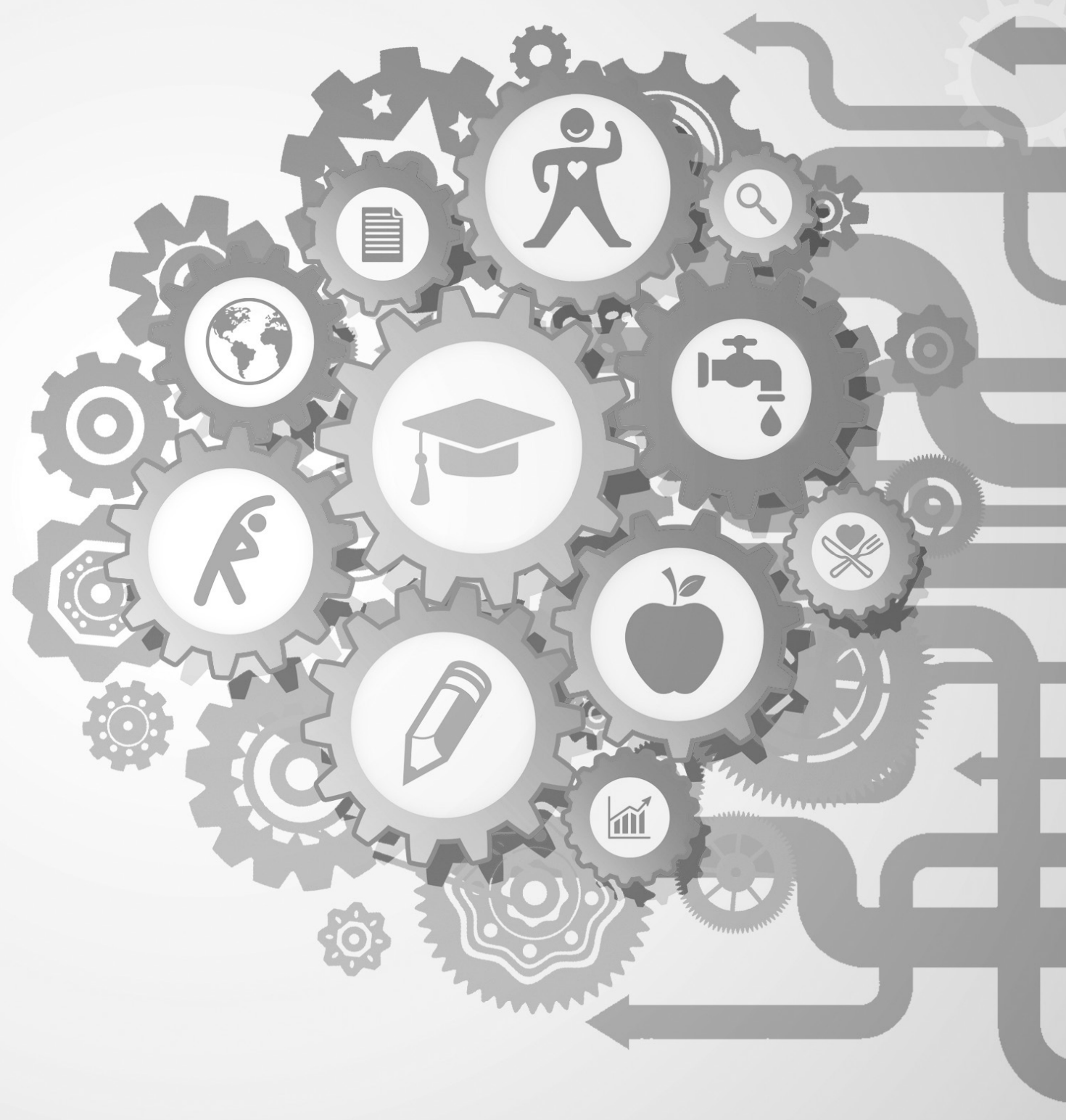





\section{Publications presented in this dissertation}

Bartelink N., van Assema P., Jansen M., Savelberg H., Willeboordse M., \& Kremers S. The Healthy Primary School of the Future: A contextual action-oriented research approach. Int J Environ Res Public Health. 2018;15(10):2243

Bartelink N, van Assema P., Jansen M., Savelberg H., Moore G., Hawkings J., \& Kremers S. Process evaluation of the Healthy Primary School of the Future: The key learning points. BMC Public Health. 2019;19(1):698.

Bartelink, N., van Assema, P., Kremers, S., Savelberg, H., Oosterhoff, M., Willeboordse, M., van Schayck, O., Winkens, B., \& Jansen, M. Can the Healthy Primary School of the Future offer perspective in the on-going obesity epidemic in young children? - A Dutch quasi-experimental study. Submitted.

Bartelink, N., van Assema, P., Kremers, S., Savelberg, H., Oosterhoff, M., Willeboordse, M., van Schayck, O., Winkens, B., \& Jansen, M. One-and two-year effects of the Healthy Primary School of the Future on children's dietary and physical activity behaviours: A quasi-experimental study. Nutrients. 2019;11(3):689.

Bartelink, N., van Assema, P., Kremers, S., Savelberg, H., Gevers, D., \& Jansen, M. Unravelling the effects of the Healthy Primary School of the Future: For whom and where is it effective? Nutrients. 2019; 11(9):2119.

Bartelink, N., van Assema, P., Jansen, M., Savelberg, H., \& Kremers, S. The moderating role of the school context on the effects of the Healthy Primary School of the Future. Int J Environ Res Public Health. 2019;16(13):2432.

\section{Other publications}

Bartelink, N., van Assema, P., Kremers, S., Savelberg, H., Oosterhoff, M., Willeboordse, M., van Schayck, O., Winkens, B., \& Jansen, M. Het effect van de Gezonde Basisschool van de Toekomst op het voedings- en beweeggedrag van kinderen: resultaten na één en twee jaar. VoedingNU. (Will be published in October 2019)

Bartelink, N., Mulkens, S., Mujakovic, S., \& Jansen, M. Long-term effects of the RealFit intervention on self-esteem and food craving. Child Care in Practice. 2018;24(1):6575. 
Bartelink, N., Jansen, M., Kremers, S., Mulkens, S., \& Mujakovic, S. Long-term effects of the RealFit intervention on body composition, aerobic fitness, and behavior. Child Obes. 2014;10(5):383-391.

Oosterhoff, M., van Schayck, O., Bartelink, N., Bosma, H., Willeboordse, M., Winkens, B., \& Joore, M. An intersectoral short-term evaluation on the social return on investment of 'The Healthy Primary School of the Future' initiative. Submitted.

Oosterhoff, M., Joore, M., Bartelink, N., Winkens, B., van Schayck, O., Bosma, H. Longitudinal analysis of health disparities in childhood. Arch Dis Child. 2019;0:1-8.

Van Kann, D., Kremers, S., Gubbels, J., Bartelink, N., de Vries, S., de Vries, N., \& Jansen, $M$. The association between the physical environment of primary schools and active school transport. Environ Behav. 2015;47(4):418-435.

Vermeiren, A., Willeboordse, M., Oosterhoff, M., Bartelink N., Muris, P., Bosma, H. Socioeconomic multi-domain health inequalities in Dutch primary school children. Eur J Public Health. 2018;28(4):610-6.

Willeboordse, M., Jansen, M., van den Heijkant, S., Simons, A., Winkens, B., de Groot, R., Bartelink, N., van Schayck, O., et al. The Healthy Primary School of the Future: study protocol of a quasi-experimental study. BMC Public Health. 2016;16(1):639.

\section{Presentations at (inter)national conferences}

Bartelink, N., van Assema, P., Kremers, S., Savelberg, H., Oosterhoff, M., Willeboordse, M., van Schayck, O., Winkens, B., \& Jansen, M. (2019). The effects of the Healthy Primary School of the Future on children's BMI z-score and dietary and PA behaviours. Oral presentation on the $5^{\text {th }}$ European Conference on Health Promoting Schools, Moscow, Russia, 20-22 November, 2019 (Upcoming).

Bartelink, N., van Assema, P., Jansen M., Savelberg, H., Willeboordse, M., \& Kremers, S. (2018). The Healthy Primary School of the Future: A contextual action-oriented research approach. Oral presentation on the $10^{\text {th }}$ IUHPE European Conference and International Forum for Health Promotion Research, Trondheim, Norway, 24-26 September, 2018. 
Kremers, S., Bartelink, N., Hermans, R., van Rinsum, C., Prevo, L., \& de Vries, N. (2018). Action-oriented research: Theory and practice of user involvement in evaluation research. Workshop on the $10^{\text {th }}$ IUHPE European Conference and International Forum for Health Promotion Research, Trondheim, Norway, 24-26 September, 2018.

Bartelink, N., van Assema, P., Kremers, S., Savelberg, H., \& Jansen, M. (2017). The Healthy Primary School of the Future. Oral presentation on the $16^{\text {th }}$ Annual Conference of the International Society of Behavioral Nutrition and Physical Activity, Victoria, Canada, 7-12 June, 2017.

Bartelink, N., van Assema, P., Kremers, S., Savelberg, H., \& Jansen, M. (2017). The Healthy Primary School of the Future. Oral presentation on the Dutch Conference for Public Health, Amersfoort, The Netherlands, 13 April 2017.

Bartelink, N., van Assema, P., Kremers, S., Savelberg, H., \& Jansen, M. (2016). The Healthy Primary School of the Future. Oral presentation on the Dutch Conference for Public Health, Rotterdam, The Netherlands, 6 April 2016.

\section{Work visits abroad}

Moscow, Russia $-5^{\text {th }}$ European Conference on Health Promoting Schools (2019, upcoming).

Trondheim, Norway $-10^{\text {th }}$ IUHPE European Conference and International Forum for Health Promotion Research (2018).

Cardiff, Wales - Two-months' work visit to the Centre for the Development and Evaluation of Complex Interventions for Public Health Improvement (DECIPHer), Department of Social Sciences, Cardiff University (2018).

Copenhagen, Denmark - Study trip: Health Promoting Schools in Denmark (2017).

Victoria, Canada $-16^{\text {th }}$ Annual Conference of the International Society of Behavioral Nutrition and Physical Activity (2017).

Glasgow, Scotland - Study trip: Health Promoting Schools in Scotland (2016).

Kuopio, Finland - Summer School: Schools for Health in Europe (SHE) (2015). 



\section{Dankwoord}

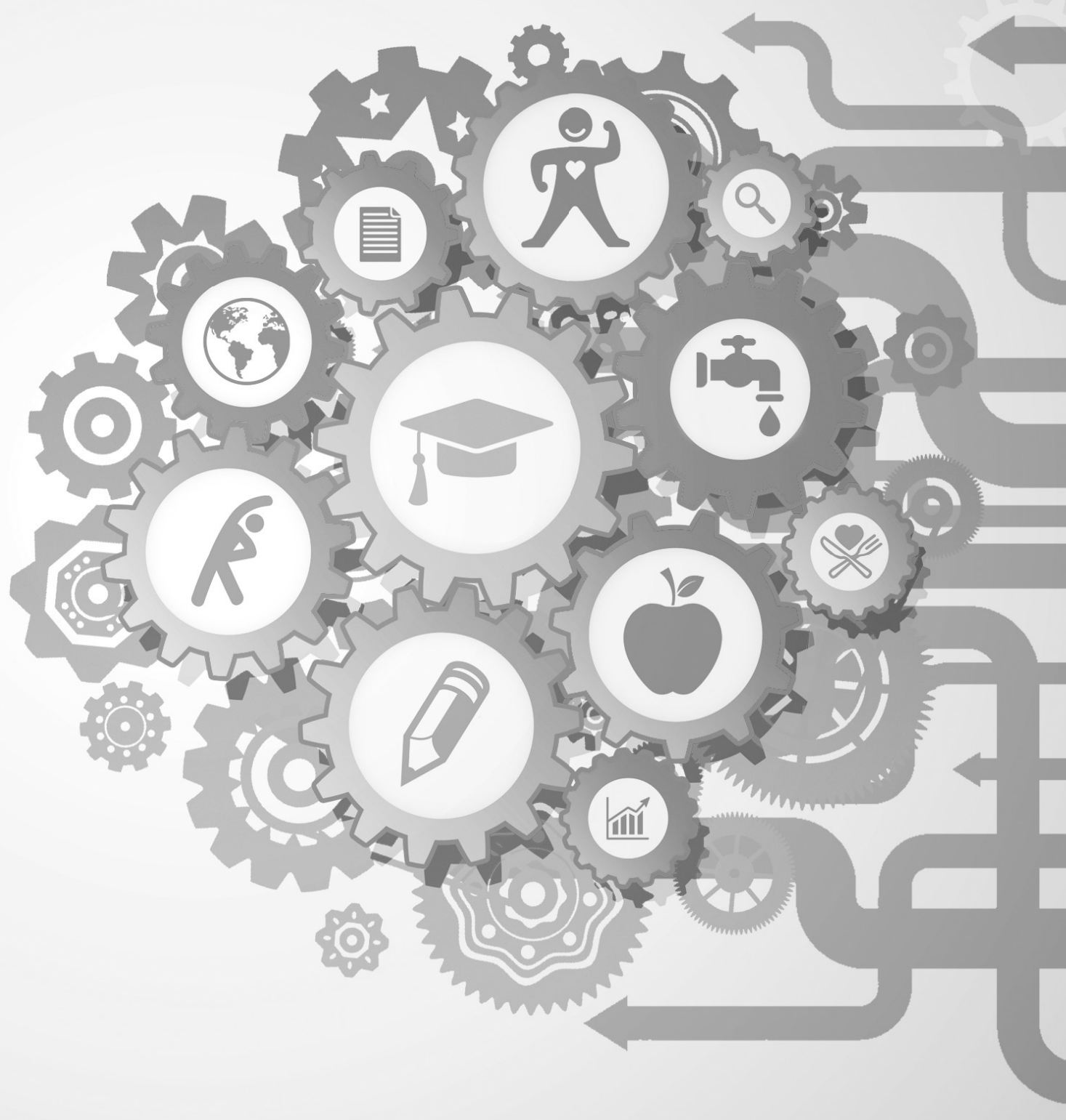


Na vijf jaar promotieonderzoek is dit toch echt het allerlaatste hoofdstuk dat ik schrijf voor in 'mijn boekje'. Het waren vijf drukke maar vooral bijzondere jaren, waarin ik veel geleerd heb en waarin vooral veel veranderd is. Ik heb er enorm van genoten, ondanks de sneltreinvaart waarmee alles ging en ik ben nog steeds blij dat ik vijf jaar geleden de keuze gemaakt heb om te gaan promoveren. Dat het zo'n fijne promotie-jaren zijn geweest heb ik zeker ook te danken aan mijn team: Patricia, Stef, Maria, en Hans. Ik kon me geen beter team om me heen wensen!

Patricia, samen hebben we heel wat uren doorgebracht tijdens onze wekelijkse overleggen. De levendige discussies waren geweldig, iets waar we allebei enorm van genoten. Al die discussies, en niet te vergeten jouw kenmerkende detail-feedback, hebben er zeker voor gezorgd dat er nu een prachtig proefschrift ligt. Je stond altijd voor me klaar en had het volste vertrouwen in me. Ik kon dan ook altijd bij je terecht, ook als het niet werk-gerelateerd was. Patricia, echt enorm veel dank voor al die fijne jaren van samenwerken en voorlopig zetten we dat nog even voort:)

Stef, ik vond het geweldig om te merken dat je me uitdaagde om de verschillende hoofdstukken van mijn proefschrift nog net even naar een hoger niveau te tillen. En ook al is het voor jou een stuk drukker geworden nu je voorzitter van de vakgroep bent, toch kon ik altijd bij je binnenlopen met vragen. Dankjewel voor al je fijne feedback, al die uitdagende prikkels, maar zeker ook voor dat onuitputtelijk enthousiasme van je en jouw positieve blik op alles. Heel inspirerend!

Maria, dé persoon van de Academische Werkplaats. We hadden al vaker samengewerkt tijdens mijn tijd bij de GGD, en altijd heb ik die samenwerking enorm leuk gevonden. Hopelijk kunnen we dat voorlopig nog wel even voortzetten! Jouw makkelijke manier van schrijven is een hele inspiratie voor mij. Daarnaast is het leuk te merken dat je altijd geïnteresseerd bent, of dat nu gaat over iets van het onderzoek of iets wat daar helemaal niks mee te maken heeft. Maria, ik ben echt blij dat je in mijn team zat!

Hans, jij stond iets verder weg van mijn onderzoek, maar was daardoor helemaal niet minder betrokken. Je keek altijd met een frisse blik naar alle stukken en je feedback was dan ook heerlijk verhelderend. Het was leuk om te merken dat we de passie voor reizen, bergen en vooral Nepal samen delen. En wat mij betreft, die Nepalese avond gaan we zeker nog een keer plannen.

Naast mijn promotieteam zijn er nog vele anderen die ik zou willen bedanken. Allereerst, de projectgroep van de Gezonde Basisschool van de Toekomst. Zonder de enorme inzet van iedereen binnen deze groep was het niet gelukt om zoiets moois neer 
te zetten. Daarom aan iedereen binnen die groep: jullie waren fantastisch! In het bijzonder wil ik de vier scholen bedanken: De Schatgraver, OBS Wereldwijs, OBS Harlekijn en Langeberg. We wisten allemaal niet hoe alles zou uitpakken en het was dan ook vaak best spannend. Ik vind het ongelofelijk bijzonder dat jullie dit avontuur zijn aangegaan.

Daarnaast wil ik iedereen bedanken die heeft bijgedragen aan alle metingen. Alle onderzoeksassistenten, stagiaires, GGD-ers, MEMIC, alle kinderen die hebben deelgenomen en hun ouders, en natuurlijk de vier interventiescholen en de vier controlescholen. Een speciaal woord van dank voor de controlescholen, voor jullie waren er namelijk helemaal geen grote veranderingen, alleen een heel team aan onderzoekers die ieder jaar de school op z'n kop kwamen zetten vanwege alle metingen. Geweldig dat jullie hierin de meerwaarde zagen en hebben willen meedoen. Zonder jullie hadden we nooit zo'n mooi onderzoek kunnen doen. De gastvrijheid die we in alle acht de deelnemende scholen hebben ervaren tijdens de metingen was hartverwarmend. We voelden ons altijd welkom en ondanks de enorme hoeveelheid aan metingen, was niks een probleem. Jullie bedankten ons zelfs op het eind van de week, waardoor het voelde als de omgedraaide wereld, wij horen jullie bedanken. Dus bij deze: enorm veel dank voor al die, ondanks drukke, toch vooral hele fijne en gezellige meetweken!

Ik wil in het bijzonder ook nog veel dank uitspreken naar de collega's van de GGD voor de support aan de scholen, de support tijdens de meetweken en de makkelijke samenwerking. Ik vond het enorm leuk om tijdens mijn promotie-traject met jullie, mijn oud-collega's, samen te kunnen werken. Jullie zagen mij helemaal niet als een externe onderzoeker maar als één van jullie, en zo heb ik dat zelf ook altijd ervaren. Ik heb er altijd van genoten en het voelde alsof we echt samen het gat tussen onderzoek en praktijk aan het dichten waren.

Maar die meetweken waren maar één onderdeel van alles. Bij het hele onderzoek waren enorm veel onderzoekers betrokken door middel van onder andere een wetenschappelijke stuurgroep, adviesraad en steungroep. Ook voor deze mensen veel dank voor alle bijdragen en input. Maar in het bijzonder het dagelijks bestuur: Onno, Andrew, Maria en Maartje. Een hele klus voor jullie om alles in goede banen te leiden, maar met een zeer mooi resultaat.

En ook Bjorn, voor jouw enorm veel dank voor alle hulp die je mij geboden hebt. Ik kon altijd bij je terecht met statistische problemen wat voor mij een enorme steun was. 
Daarnaast wil ik in het bijzonder nog enkele andere PhD-ers van de Gezonde Basisschool van de Toekomst bedanken: Marije, Renate en Elise. Ik vond het enorm leuk om samen, ieder op zijn eigen manier, het PhD traject te doorlopen bij de Gezonde Basisschool. Ik vond het altijd erg gezellig om weer lekker bij te kletsen onder het genot van een hapje of een drankje. Dat er nog maar veel van dat soort bijklets-momentjes mogen volgen in de toekomst, ook al zitten we ondertussen volledig verspreid over Nederland.

I also would like to thank the DECIPHer group in Cardiff, Wales, and especially Graham and Jemma. I had such a great time with you and I felt so welcome at DECIPHer. It was nice to meet again in Trondheim and to have our little reunion. Let's hope more of those will follow.

Ik wil ook nog graag mijn collega's van de vakgroep bedanken voor de gezelligheid en de altijd fijne werksfeer. In het bijzonder wil ik mijn (oud-)kamergenootjes Teun, Celeste, Dorus en Yil bedanken. Stuk voor stuk was het fijn om met jullie een kamer te delen, en om maar in de woorden van Dorus te spreken, samen op één kamer een beetje te hobbyen.

Ook wil ik mijn niet twee, maar drie paranimfen bedanken.

Celeste, ook al kun je er tijdens mijn verdediging niet bij zijn, je zult toch altijd één van mijn paranimfen zijn. Wat hebben we een enorm leuke vriendschap opgebouwd al die jaren en wat hebben we veel met elkaar gedeeld. Ik heb je die laatste maandjes van mijn PhD dan ook echt gemist als kamergenootje. Maar we houden contact, want ik wil graag die kleine Jesper zien opgroeien. En nu je mijn bruidsboeket gevangen hebt, verwacht ik ook nog wel een bruiloft in de toekomst!

Dennis, ook wij kennen elkaar al vanaf het begin van mijn PhD. Het is altijd gezellig met jou, zeker voor wat betreft het kletsen over vakanties en (de voorbereidingen op) een actieve prestatie, we wisten elkaar altijd te vinden. Echt leuk dat jij nu met CARA aan de slag gaat en we wellicht nog wat samen kunnen werken aan een onderzoek. En Celeste en Dennis, wat betreft dat boulderen, dat moeten we wel echt snel nog eens gaan doen.

Marije, ik vind het super leuk dat jij ook mijn paranimf bent. Wat vond ik het altijd fijn om met jou samen te werken. En vooral onze data-cleaning-dagen met carnaval zal ik niet vergeten. Ook al was het toen en op heel veel andere momenten, keihard doorwerken, het was ook altijd erg gezellig. Ik vind het echt leuk dat ik het hele traject zo samen met jou doorlopen heb, zelfs tot aan mijn laatste etappe, mijn verdediging. 
Tenslotte wil ik graag al mijn lieve vrienden en (schoon)familie bedanken. Altijd hadden jullie interesse in mijn onderzoek en probeerden jullie te snappen wat ik aan het doen was. Ook al leek het soms voor jullie dat ik alleen maar op vakantie ging... Ga je nu ALWEEER naar het buitenland?!

Pap, mam, jullie wil ik in het bijzonder nog bedanken. Ik voelde me altijd enorm gesteund door jullie. En het is ontzettend leuk om te zien en horen hoe jullie vol trots aan anderen vertellen over mijn onderzoek. Ook de interesse was er altijd, en zelfs voor de ingewikkelde Engelse artikelen vonden we een oplossing: gewoon zin voor zin vertaalde ik wat er stond, zodat de inhoud echt duidelijk werd. Ontzettend fijn om zo'n lieve ouders te hebben!

En dan nu de allerbelangrijkste persoon in mijn leven. Lieve Jorik, wat ben ik blij dat ik jou ontmoet heb en wat hebben we het fijn samen. Ongelofelijk hoeveel kan gebeuren tijdens een PhD, van elkaar leren kennen tot aan samenwonen, een huis kopen en nu ook nog sinds afgelopen zomer getrouwd. Je wist me altijd te helpen op net die manier die ik nodig had: het bieden van de nodige afleiding door er samen op uit te trekken, gewoon een bord warm eten, Engelse vertaalhulp of een knuffel en een hart onder de riem dat ik goed bezig was. Lieverd, je bent echt een enorme steun en toeverlaat voor me geweest dit hele traject, nog veel meer dan je zelf denkt. Ik hou van je! 



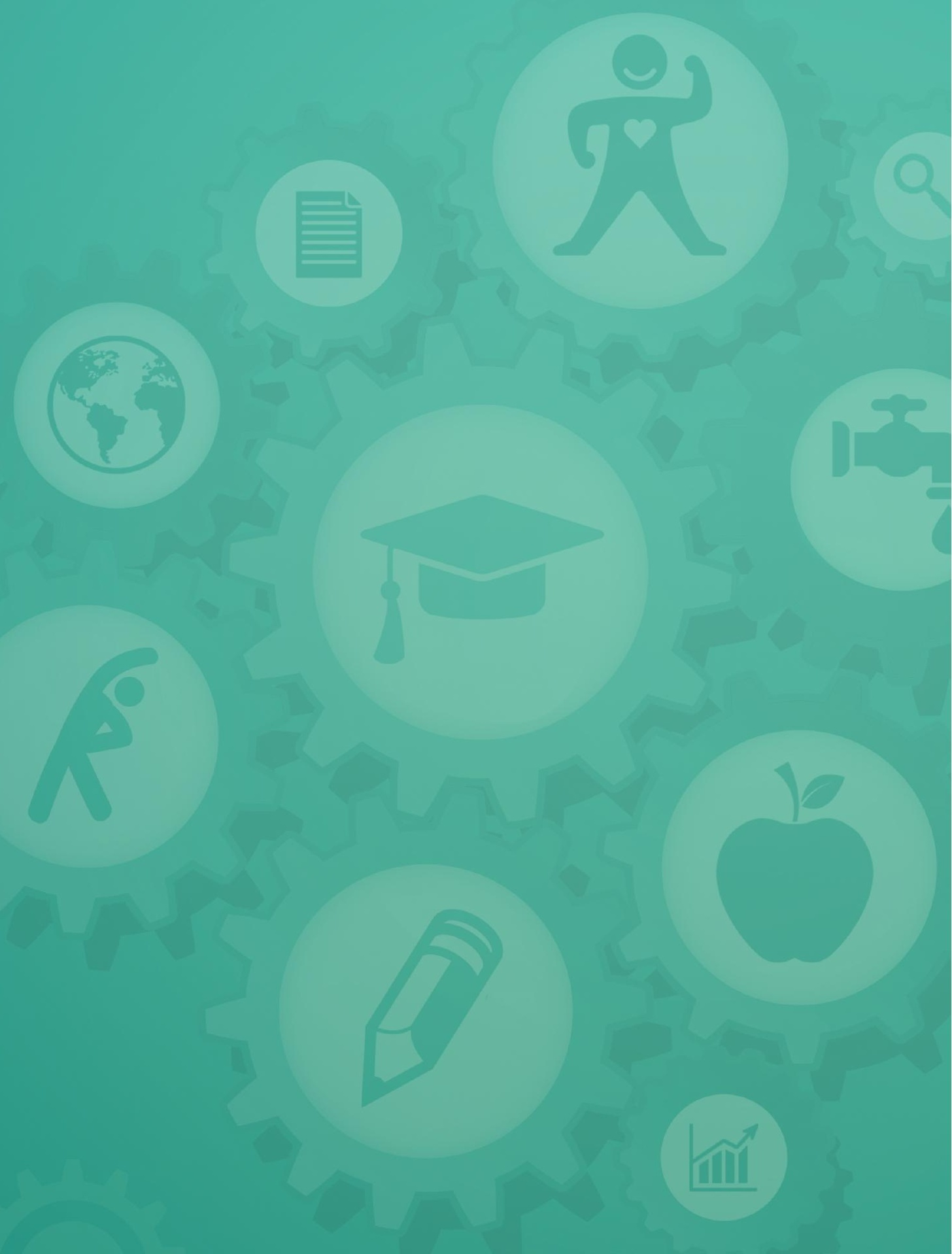

Evaluating health promotion in complex adaptive school systems:

The Healthy Primary School of the Future 